\title{
Trophic interactions of ants, birds and bats affecting crop yield along shade gradients in tropical agroforestry
}

\author{
Dissertation \\ zur Erlangung des Doktorgrades \\ der Mathematisch-Naturwissenschaftlichen Fakultäten \\ der Georg-August-Universität Göttingen \\ im Promotionsprogramm \\ Biologische Diversität und Ökologie

$$
\begin{aligned}
& \text { vorgelegt von } \\
& \text { Pierre Gras }
\end{aligned}
$$$$
\text { geboren in Peine }
$$ \\ Göttingen, November 2014
}





\section{Promotionsausschuss:}

Prof. Dr. Teja Tscharntke (Agrarökologie, Georg-August-Universität Göttingen)

PD Dr. Yann Clough (Centre for Environmental and Climate Research, Lund University)

Prof. Dr. Stefan Vidal (Agrarentomologie, Georg-August-Universität Göttingen)

Tag der mündlichen Prüfung: 10.12.2014 



\section{$\underline{\text { Contents }}$}

Chapter 1: The value of tropical agroforestry for ecosystem service provision, nature conservation and agricultural productivity.............................................. 9

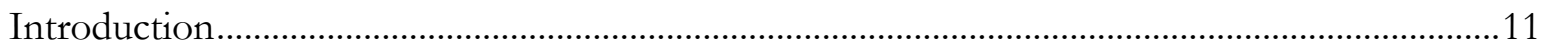

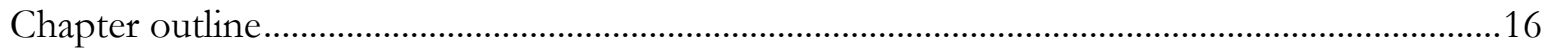

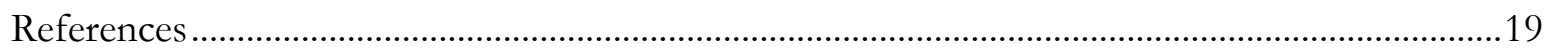

Chapter 2: How ants, birds and bats affect crop yield along shade gradients in tropical agroforestry ................................................................................ 23

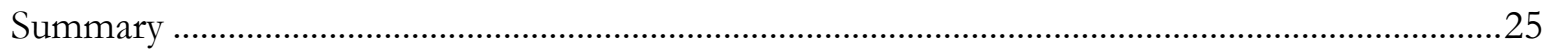

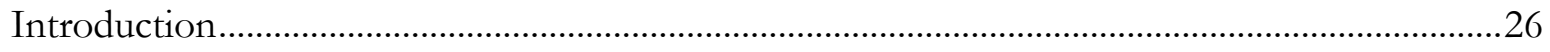

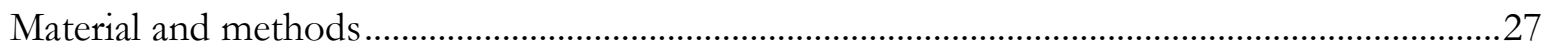

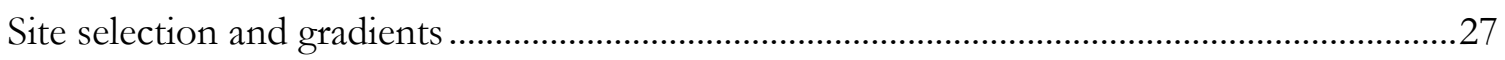

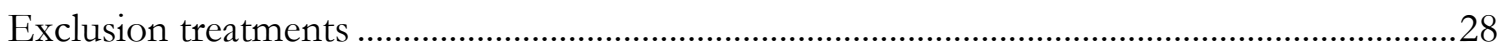

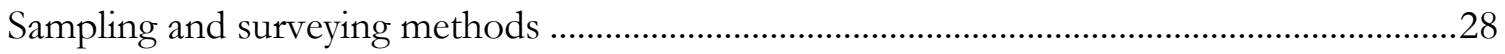

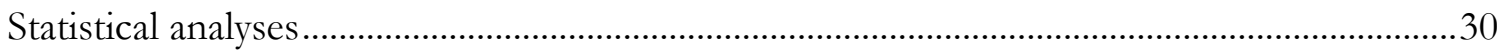

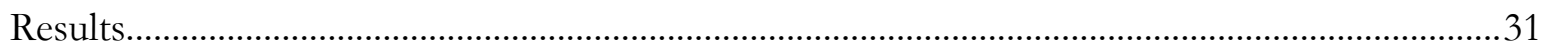

Effects of predator exclusion, canopy cover and distance to forest on cacao yield..................31

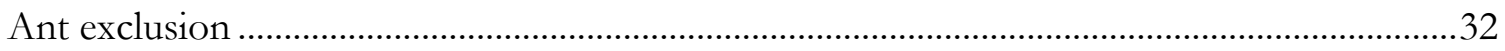

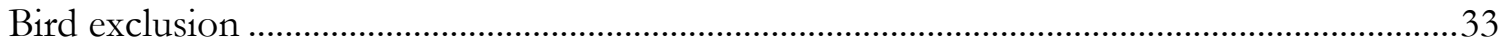

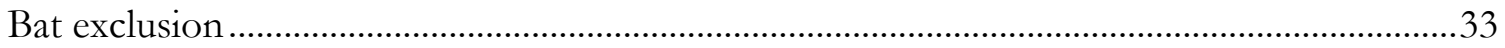

Ant and bird diversity along the canopy cover and forest distance gradient ............................35

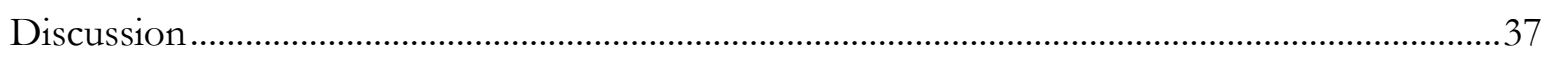

Canopy-cover dependent effects of ants ..................................................................................... 37

Bird exclusions can decrease or increase yields depending on canopy cover.............................38

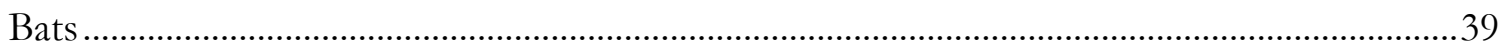

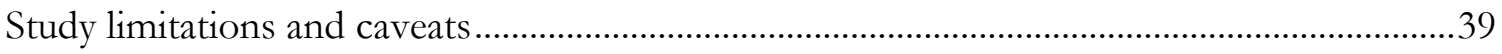

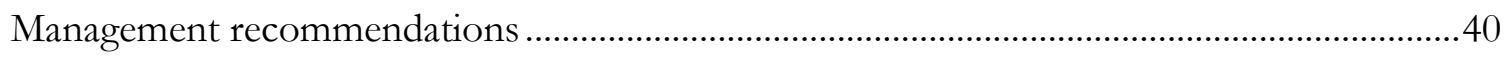

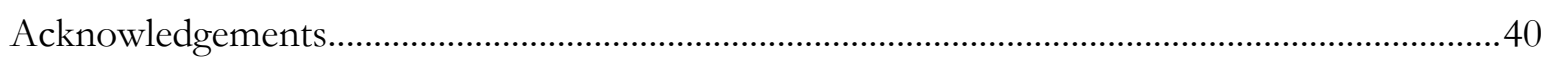

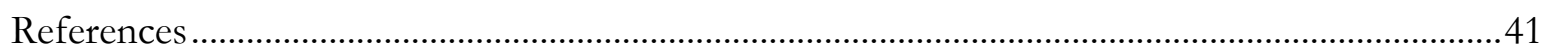

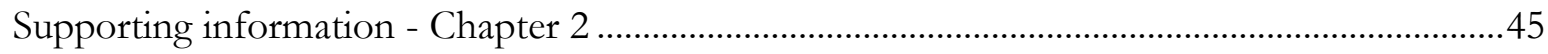

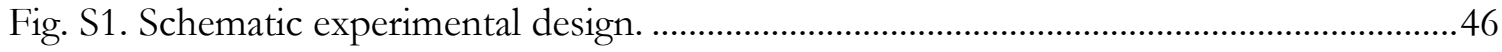

Table S2. Non-crop tree species within chemical application free area.....................................4

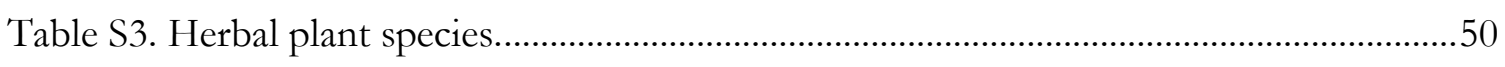

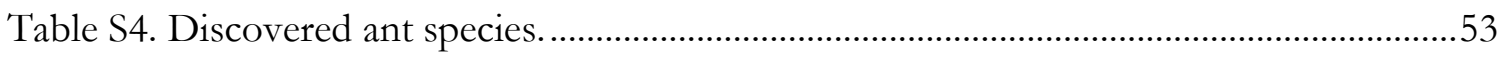

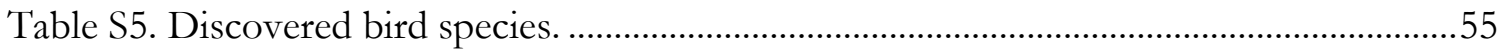

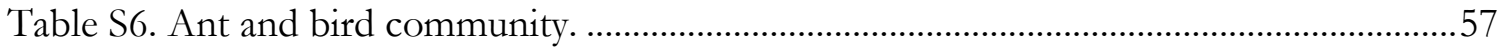

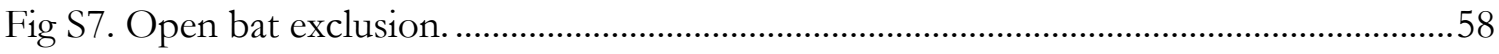

Fig. S8. Conical-shaped ant exclusion rings (without insect glue) ...........................................59 
Table S9. Potentially yield influencing variables (additional to the predator exclusion).........60

Table S10. Characteristics of experimental trees and plantations at treatment level...............63

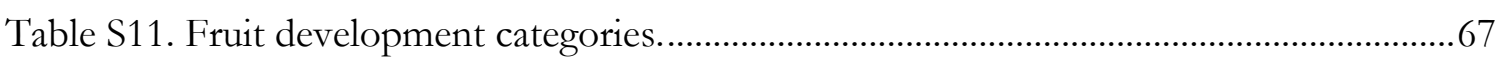

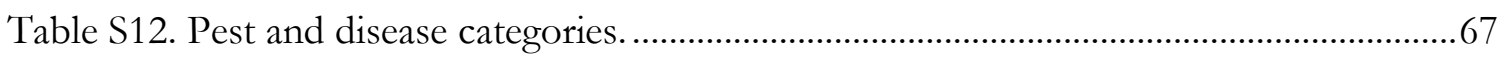

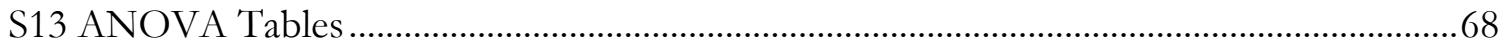

Fig. S14. RDA - Management, landscape, and predator access manipulation influences on the ant community composition (morpho species level) ....................................................................86

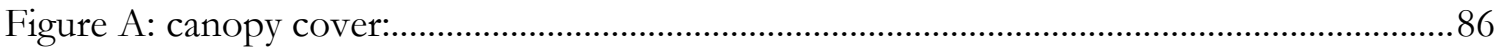

Fig. S15. RDA - Management and landscape effects on the bird community (species level).

Fig. S16. Visualization of bird and ant community composition related to shade cover and

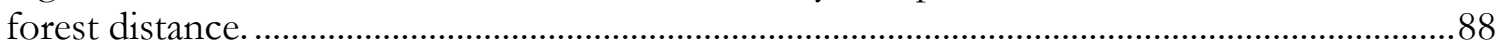

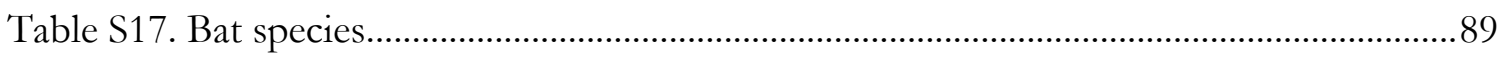

Table S18 Agroforest description ........................................................................................... 91

Chapter 3: Resource distribution changes species-specific trophic positions and ecosystem service provision of omnivorous ants.............................. 93

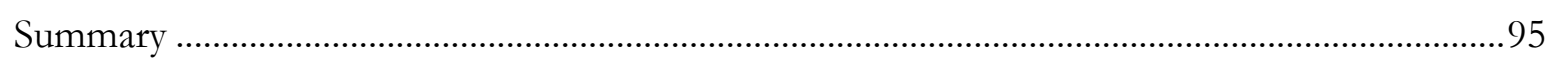

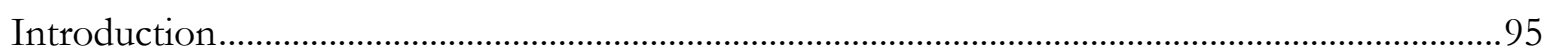

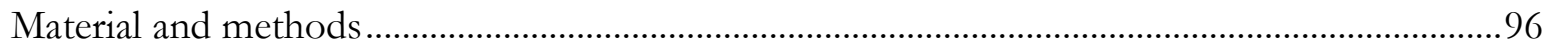

Study sites and ant colony establishment................................................................................. 96

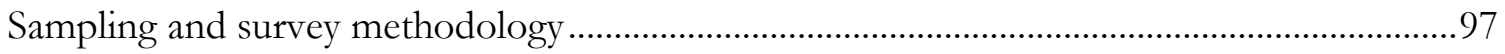

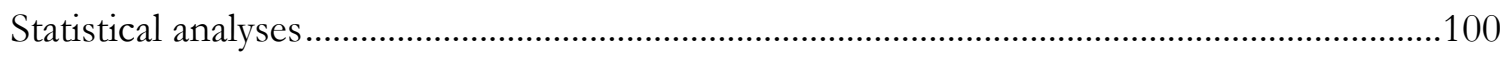

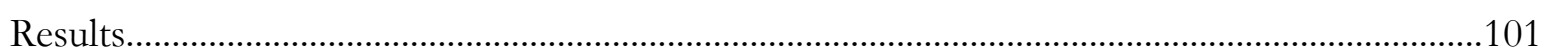

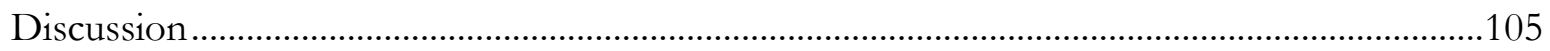

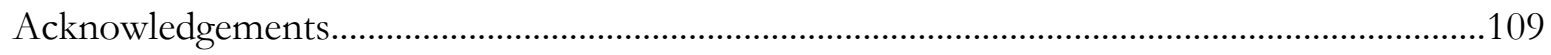

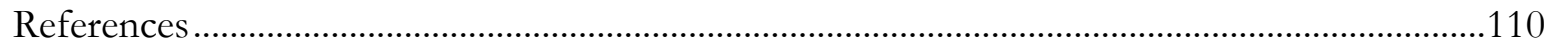

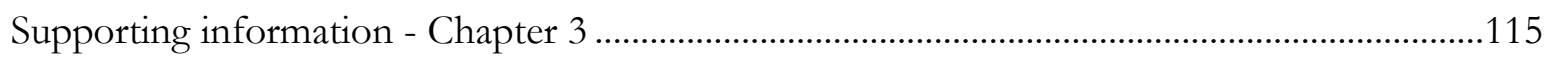

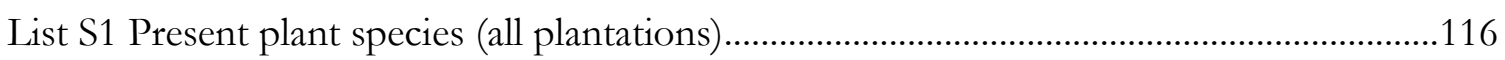

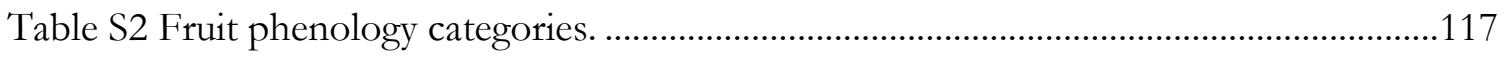

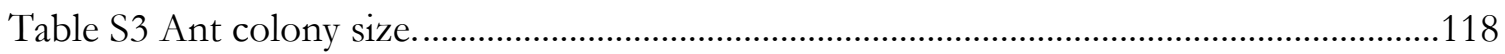

Description S4 Pre-experiment life cycle $D$. thoracicus and P. cf. cordata.................................118

Chapter 4: Multi-scale intensification changes trophic position of omnivores in tropical agroforestry ................................................................................. 119

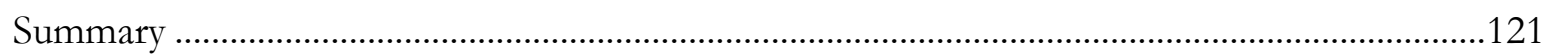

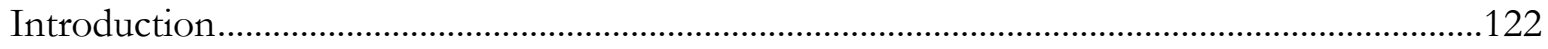

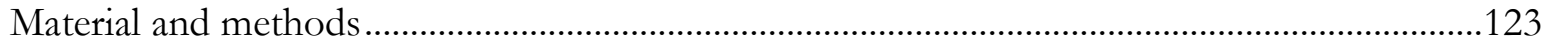

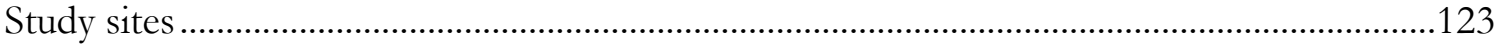

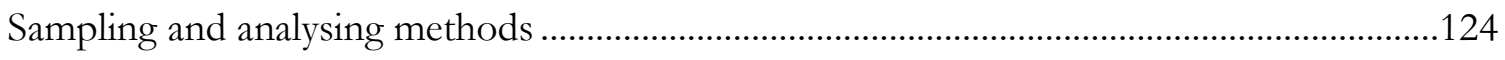

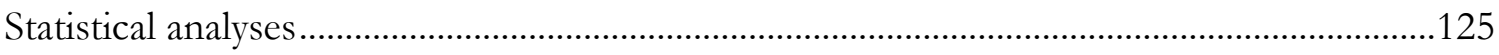


Results

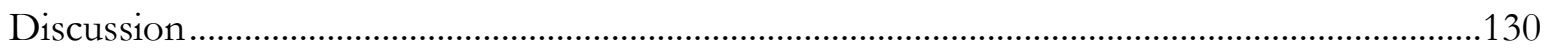

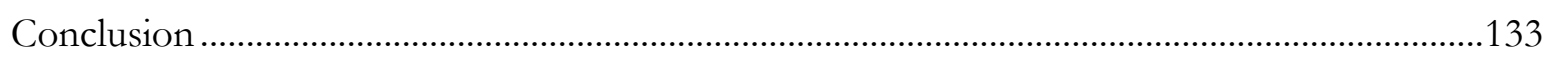

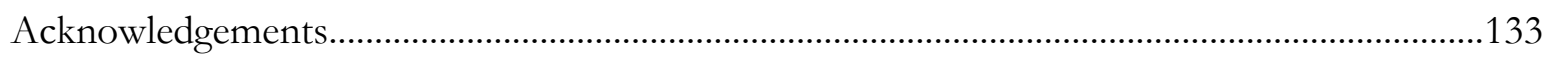

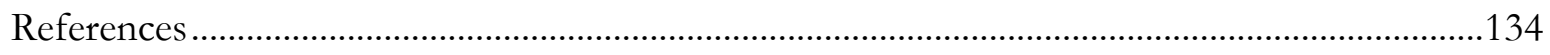

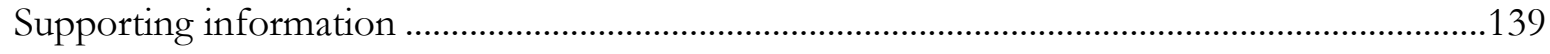

Supporting information - Chapter 4 .............................................................................................140

Fig. S1. Unstandardized $\delta 15 \mathrm{~N}$ values of all ant (morpho-) species........................................141

Fig. S2. Unstandardized $813 \mathrm{C}$ values of all ant (morpho-) species...........................................142

Chapter 5: Intra-specific plasticity in dietary resource-use maintains interspecific complementarity in natural ant communities ...................................145

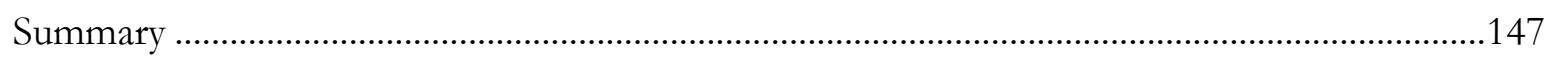

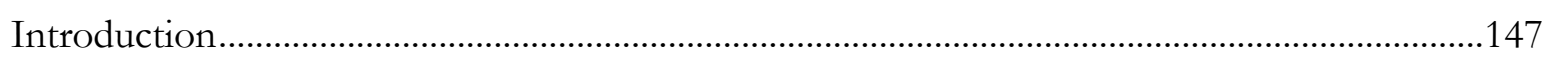

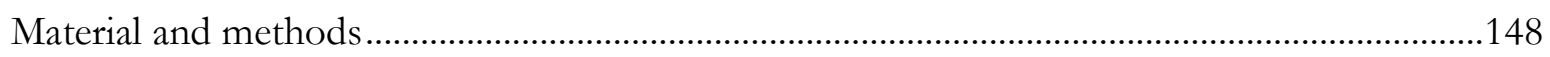

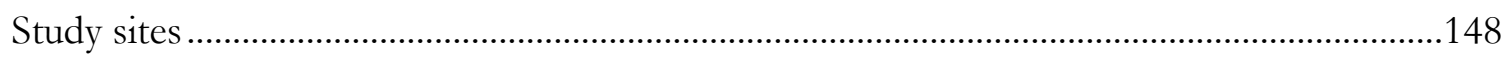

Sampling and analysing methods ............................................................................................149

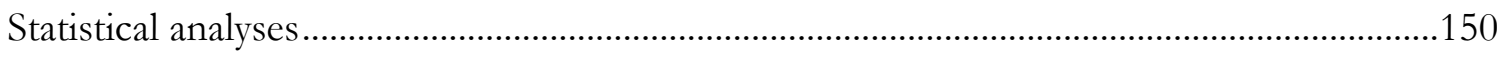

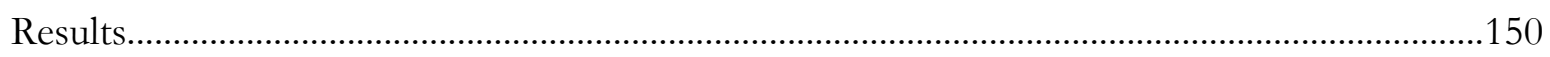

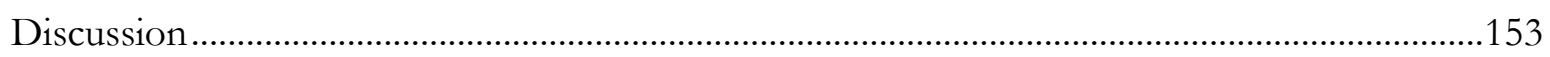

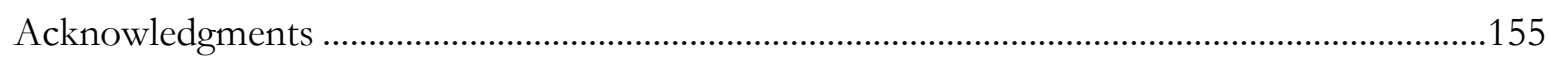

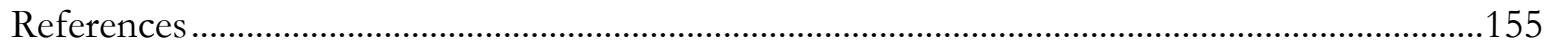

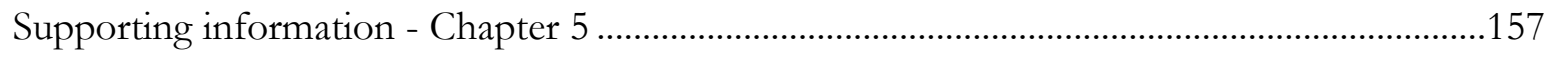

Thesis summary .........................................................................159

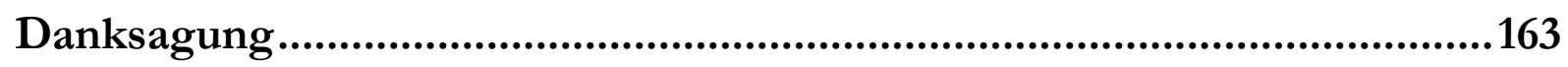

Curriculum vitae ..................................................................................165

Thesis declarations ...................................................................... 167 
8 / 168 
Chapter 1: The value of tropical agroforestry for ecosystem service provision, nature conservation and agricultural productivity

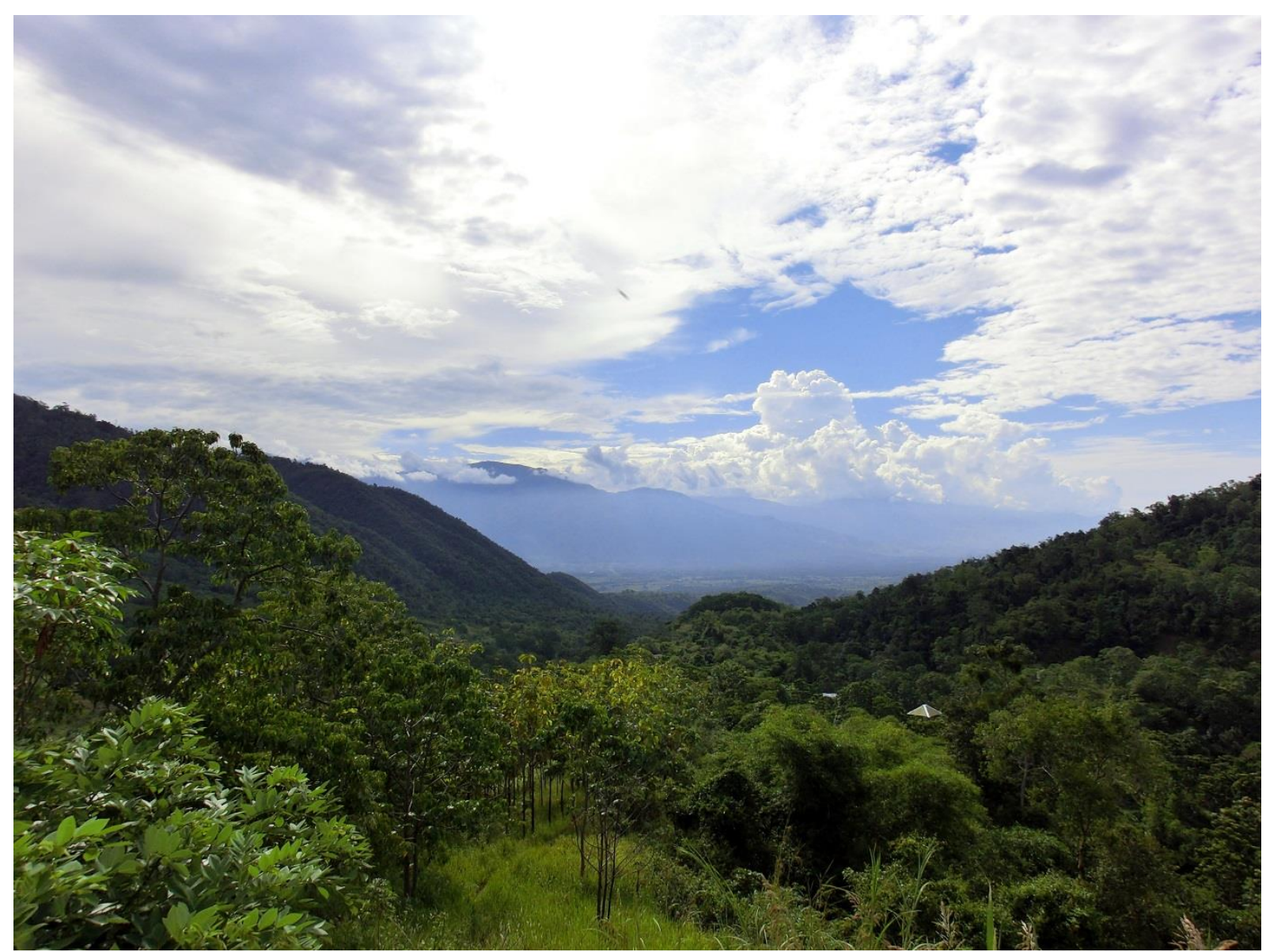

The Palolo valley harbours a hilly, agroforestry-dominated landscape with large paddy fields in the flat areas. 
$10 / 168$ 


\section{Introduction}

Agricultural landscapes cover 40 - $60 \%$ of the world's terrestrial surface and agricultural expansion is one major reason for deforestation in the tropics during the early $21^{\text {th }}$ century (Angelsen 1995, Geist and Lambin 2002, Achard et al. 2002, Millennium Ecosystem Assessment 2003, 2005, Naeem et al. 2009, Power 2010). Mostly, local economic decisions enhance deforestation, regardless of the effects on biodiversity, ecosystem functions, and ecosystem services (Geist and Lambin 2002, Balvanera et al. 2006, Hooper et al. 2012). For a long time, humans have benefited from natural ecosystems through services such as freshwater supply, erosion limitation, recreational value, or pest control and pollination. If pest control or pollination vanish, the production cost of crops will rise, given that these services alter crop yields and directly link nature to human well-being (Loreau and Hector 2001, Mooney et al. 2004, Costanza et al. 2007, Gallai et al. 2009, Power 2010, Robertson and Wainwright 2013). Consequently, sustainable land-use concepts, including ecosystem service provision, need to be developed in order to maintain agricultural production at affordable economic costs in the long term.

Many organisms living in natural and semi-natural ecosystems have been reported to provide ecosystem services (Costanza et al. 1997, Daily 1997, Millennium Ecosystem Assessment 2005, Fisher et al. 2009, Nelson et al. 2009, Power 2010, Cardinale et al. 2012). Tropical rainforests harbour many of these organisms, but agrarian landscapes can also house a diverse and abundant set of animals and plants (Perfecto et al. 1997, Myers et al. 2000, Cardinale et al. 2012). Moreover, tropical agricultural systems can provide a high quality matrix and facilitate animal movement between natural patches of vegetation (Perfecto and Vandermeer 2008). However, ongoing agricultural intensification threatens almost all kinds of biological communities in agroecosystems (Matson 1997, 2013, Tscharntke et al. 2005, Philpott and Armbrecht 2006, Fischer et al. 2008, Wanger et al. 2010, but see Marín et al. 2013).

Agricultural products from tropical countries such as cacao, coffee and palm oil are in highly demanded in the world markets, thus tropical countries - e.g. Indonesia - supply these goods in annually increasing amounts (Rice and Greenberg 2000, Geist and Lambin 2002, Franzen and Borgerhoff Mulder 2007, Germer and Sauerborn 2007, Clough et al. 2009b). To increase crop productivity and maximize the income of farmers, agricultural intensification is the most obvious solution. In cacao production systems, common intensification practices are shade tree removal, fertilizer application, and chemical applications (pesticides, herbicides, and fungicides). Moreover, freshly logged and burned rainforest areas have fertile soils demanded to create new cacao plantations (Rice and Greenberg 2000, Franzen and Borgerhoff Mulder 2007). Many cacao farmers grow intensively managed full-sun cacao, which harbours a low biodiversity due to shade tree removal and comprehensive chemical applications (Rice and Greenberg 2000, Franzen and 
Borgerhoff Mulder 2007). Compared to shade-cacao, sun-cacao plantations provide little canopy cover resulting in a hot and dry microclimate as well as fewer tree and herb species (Rice and Greenberg 2000b, personal observation). The removal of shade trees may decrease the nesting-site availability for ant species and the diversity of bird communities (Philpott and Foster 2005, Armbrecht et al. 2006, Clough et al. 2009a), leading to ant communities dominated by a few very abundant species, e.g. Anoplolepis gracilipes (SMITH, 1857) (Bos et al. 2008). In addition, microclimatic conditions and the dominance of ecologically important species were identified as major drivers of ant species diversity in agroforests (Wielgoss et al. 2010).

Ecosystem services and disservices such as pest control or disease distribution are the direct or indirect result of the supplier's trophic role (Tilman et al. 2009, Power 2010, Cardinale et al. 2012, Martin et al. 2013). Birds and bats fulfil many ecological roles as predators, pollinators, scavengers (only birds), parasites, seed dispersers, seed predators, ecosystem engineers and prey. However, they differ in their diurnal activity patterns and their prey spectrum (Whelan et al. 2008, van Bael et al. 2008, Wenny et al. 2011). In the tropics, ants can represent up to $80 \%$ of the insect biomass and conduct similar trophic functions as birds and bats, but at lower trophic levels (Hölldobler and Wilson 1990, Brown 2000). Furthermore, omnivorous ants can shift their feeding strategy between mainly cryptic-herbivorous and mainly predacious and vice versa (Brown 2000, Philpott and Armbrecht 2006). Omnivorous ants e.g. Dolichoderus thoracicus (SMITH, 1860) and Philidris cf. cordata (SMITH, 1859) can increase crop yield, through predation, or impair yield, through disease distribution and cryptic-herbivory (Wielgoss et al. 2014). The feeding strategy of these ants cannot be explained in general, since in order to account for a very high biomass in an ecosystem, ants have to be cryptic-herbivores to maintain their biomass (Hunt 2003, Davidson et al. 2003). Although ants act as very abundant and active predators shaping arthropod communities in the tropical forest canopy (Floren et al. 2002). Whether shifting between herbivorous and predatory foraging strategies is species-specific, environment-mediated or partly both is still unclear. Food webs in agroecosystems are complex, and researchers need to investigate multi-trophic interactions in detail to understand these ecosystems (Duffy 2002). Thus, the assessment of direct and indirect trophic interactions can be crucial to understand disease distribution and yield formation in tropical agroforestry (Vandermeer et al. 2002, 2010, Mooney et al. 2004, Wielgoss et al. 2012). Especially, predatory functions of ants are important services in tropical agricultural systems and, at least partly, functions change according to abiotic conditions and antagonistic, additive or synergistic interactions with other organisms.

The agricultural system discussed in this thesis are cacao agroforestry. We conducted the experiments within an anthropogenic landscape at the eastern edge of the Lore Lindu National Park (Central Sulawesi, Indonesia). The chosen regions, namely the Napu valley and the Palolo 
valley, are dominated by cacao agroforests, paddy fields and, especially in the Napu valley, vegetable production (e.g. tomato, cucumber, cabbage and chili). Within Indonesian cacao plantations the moth Conopomorpha cramerella (SNELLEN, 1904) (Gracillariidae) and the bug Helopeltis sulawesi (STONEDAHL, 1991) (Miridae) are supposed to be the major pests. H. sulawesi adults and nymphs pierce fruit or leaf surfaces to suck plant sap. Thereby the bug induces surface necrosis. The moth C. cramerella lays eggs on fruit surfaces and hatching larvae drill inside the fruits to feed on fruit pulp and developing seeds. Damage of C. cramerella is hardly visible before harvest (see Fig. 3). Beside these two major pests, various caterpillars feed on cacao flowers and fruits (see Fig. 4), other herbivores such as beetles, bugs, mealybugs and aphids are also frequent visitors of cacao trees (species selection see Fig 5). Mesopredators in cacao agroforests are very diverse as well. Common mesopredators are ants, earwigs, spiders, lacewings, lady beetles, assassin bugs, and various parasitoids (see Fig 6).

Here, we studied predator (birds and bats) and mesopredator (ants, partly spiders) mediated ecosystem services (biocontrol of pests), predator-mesopredator interactions and specific trophic roles of ants in cacao plantations (Fig. 2) along gradients of canopy cover (Fig. 1) and distance to forest margins (Chapter 2, Fig. 1).

We asked the following questions:

- Does the exclusion of ants, birds and bats affect cacao yields, and if so, do effects change with landscape context (distance to forest margins) or local context (shade management), and are these effects synergistic, additive or antagonistic?

- What are the likely processes explaining the impact of the exclusion of bats, ants or birds on yield (e.g. pest and disease infestation, fruit set or fruit abortion)?

- Do ants respond to spatiotemporal food resource distribution by density adaptations or adapted foraging strategies (e.g. switching from mainly predatory to mainly crypticherbivory), and is this response different between two ecologically dominant ant species (D. thoracicus and P. cf. cordata)?

- Does shade tree cover and distance to forest margins affect the community-level trophic position of omnivores, and if so, does species turnover or trophic plasticity leads to changes along these two gradients?

- Do ants adept resource-use in response to the presence of trophically similar species within a local community? 


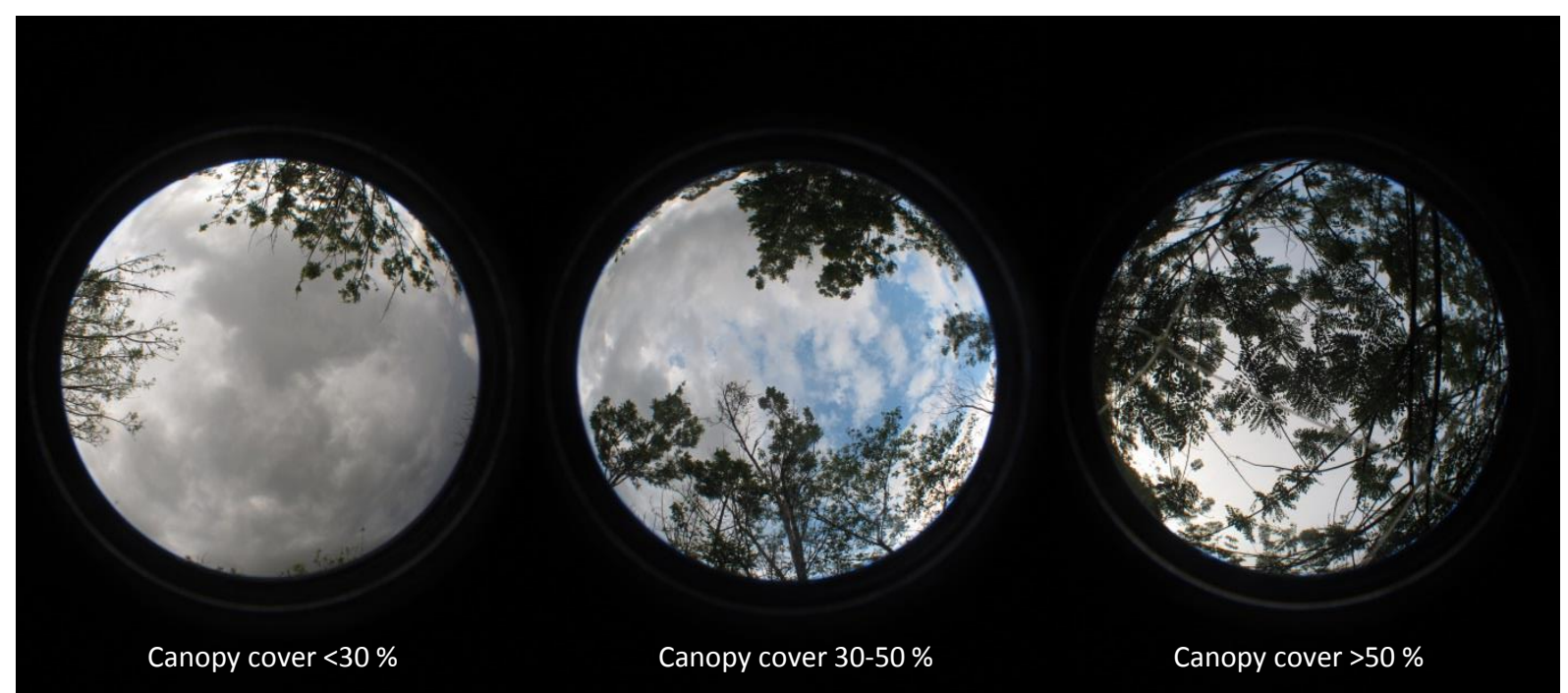

Fig. 1. Canopy cover gradient (fish-eye pictures).

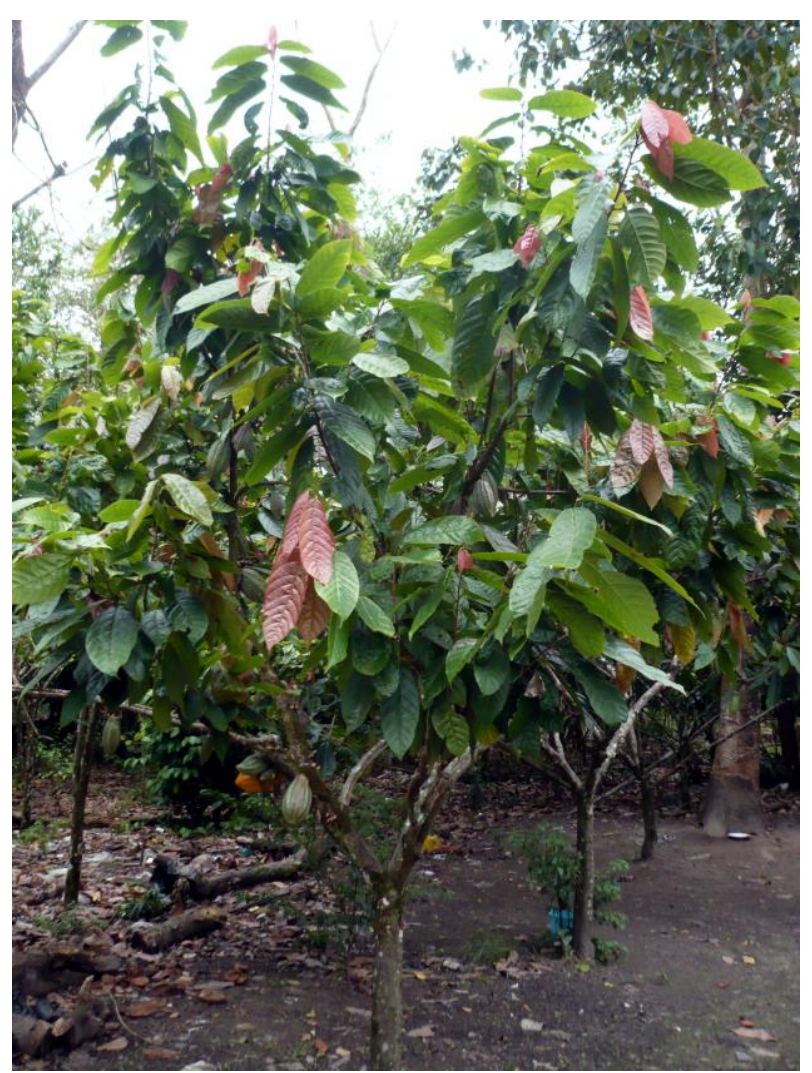

Fig. 2. Cacao trees (Theobroma cacao L.) recently fruiting, showing the typical branching point and flushing young leaves (red leaves). 


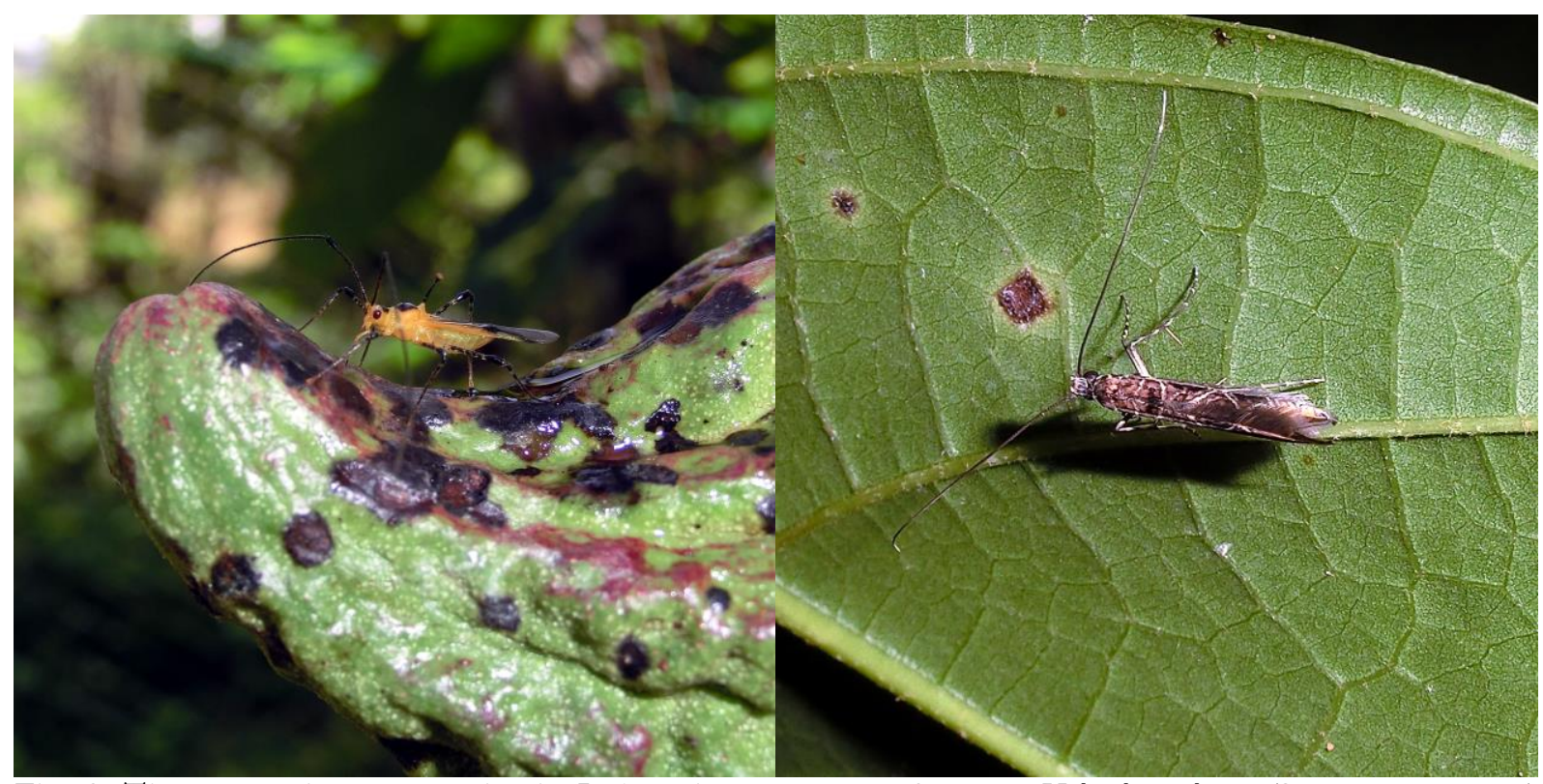

Fig. 3. The two main pest species in Indonesian cacao agroforestry. Helopeltis sulawesi (STONEDAHL, 1991) (Miridae) (left) and Conopomorpha cf. cramerella (SNELLEN, 1904) (Gracillariidae) (right).

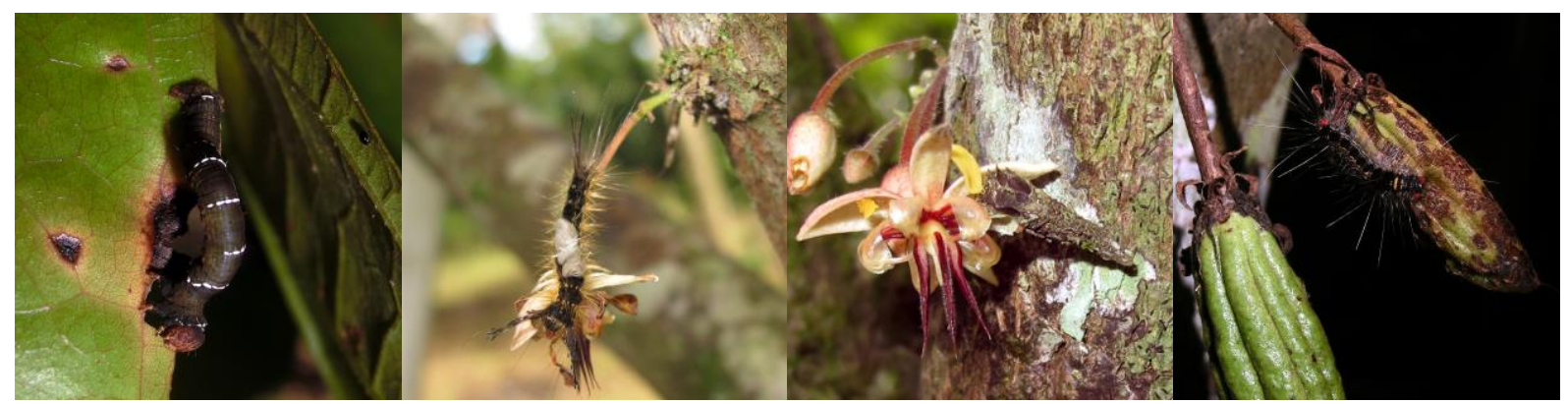

Fig. 4. Flower and fruit herbivores found in cacao agroforests in Central Sulawesi, Indonesia. Starting left: Geometridae sp., Lymantriidae sp., Psychidae sp., and Notodontidae sp..

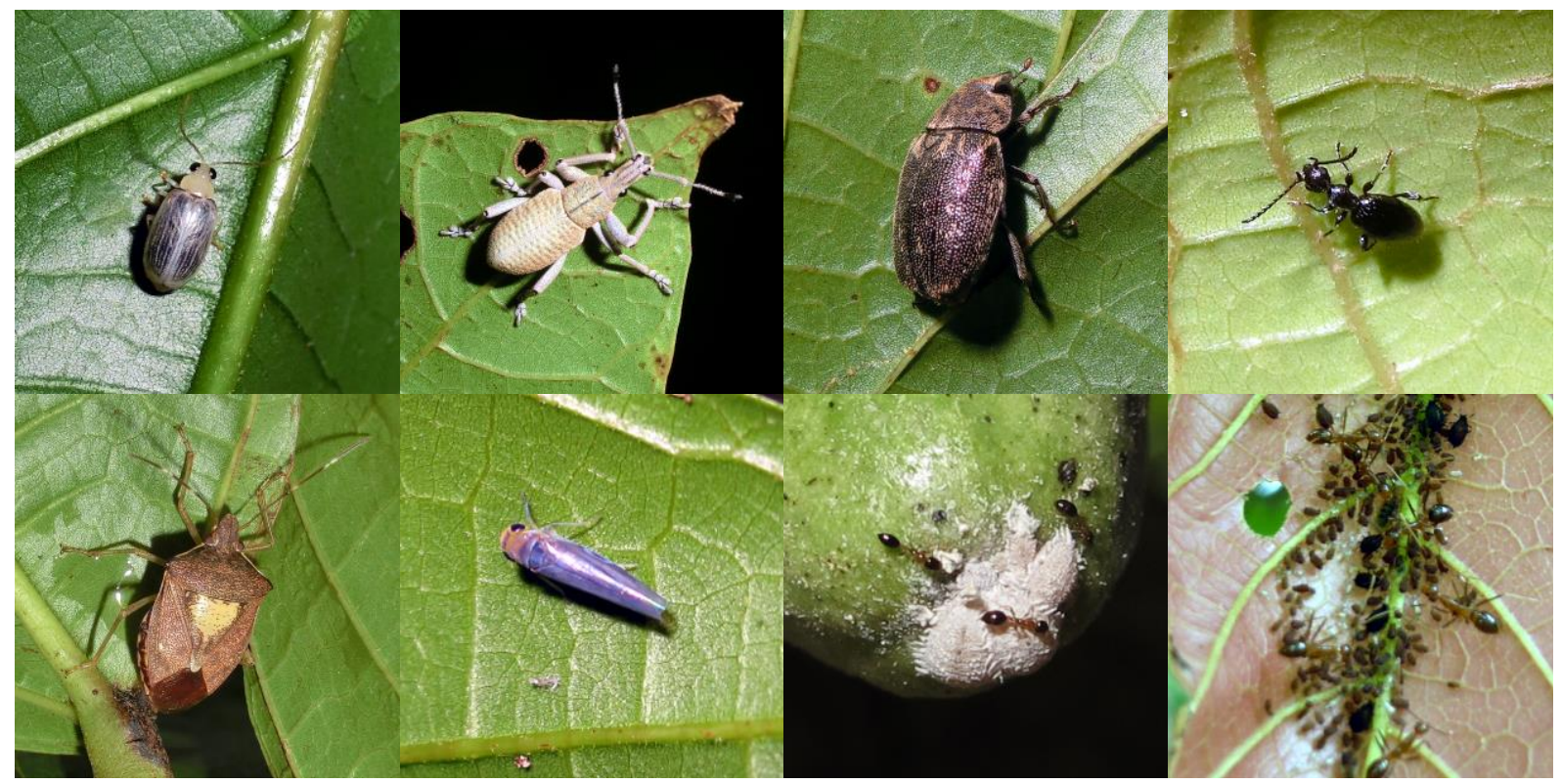

Fig. 5. Other herbivores found in cacao agroforests in Central Sulawesi, Indonesia. Starting top-left: Monolepta sp. (Chrysomelidae), Curculionidae sp., Apogonia sp. (Scarabidae), Anthicidae sp., Pentatomidae sp., Cicadellidae sp., Pseudococcidae sp., and Aphidoidea sp.. The two last mentioned taxa are the most common ant trophobionts. 


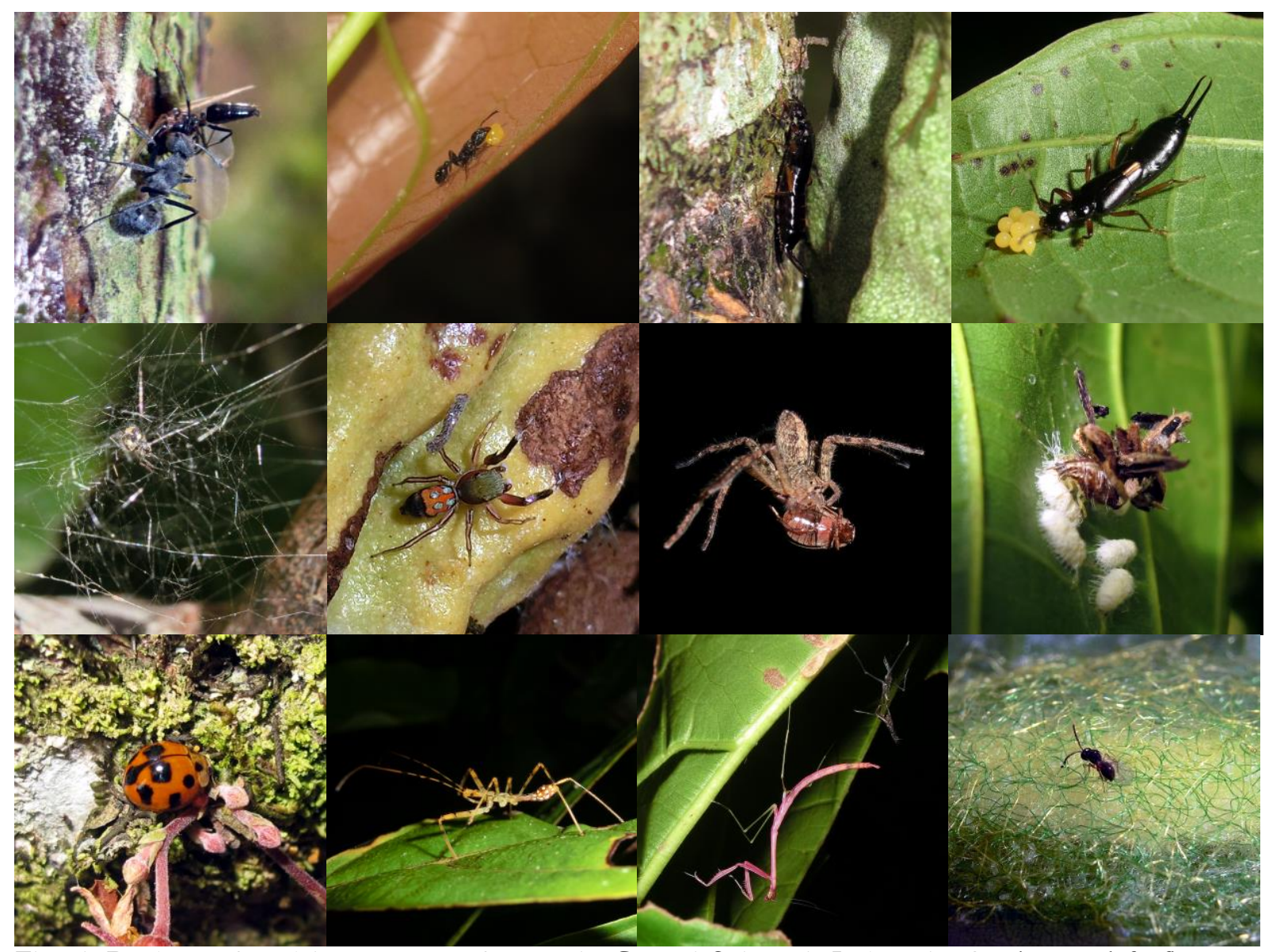

Fig. 6. Predators found in cacao agroforestry in Central Sulawesi, Indonesia. Starting top-left: first row: Polyrhachis cf. dives carries hymenoptera (SMITH, 1857), Tetramorium sp. feeding egg, Dermaptera spp. one hidden between fruits, the other feeding eggs, second row: small web spider in cacao branch triangle, jumping spider on cacao fruit, big hunting spider holds Scarabidae, Neuroptera sp. larvae feeding eggs, Coccinellidae sp. searching aphids close to flowers, Reduviidae sp., Mantodea nymph, parasitic hymenoptera on cocoon.

\section{Chapter outline}

Chapter 2: How ants, birds and bats affect crop yield along shade gradients in tropical agroforestry.

This chapter aims at investigating interactive effects of ants, birds and bats on cacao yield formation along canopy cover and forest distance gradients.

Using a large-scale predator exclusion experiment, we showed that optimal cacao yield occurred under 30 - $40 \%$ canopy cover. Exclusion of ants led to strong yield reductions, yet only under medium and low canopy cover $(<45 \%)$. Exclusions of birds resulted in reduced yields under high canopy cover $(>40 \%)$, but increased yield under low canopy cover $(<30 \%)$. Effects of ant and bird exclusions were additive, while bat exclusion did not affect yield. The absence of birds led to reduced numbers of harvested fruits, while exclusion of ants impaired fruit size as well as number of harvested fruits. The arthropod community changed due to predator exclusion suggesting that reduced yields were associated with increased pest damage. Mesopredator release (i.e. spider 
abundances) was stronger when birds rather than ants were excluded, explaining the enhanced pest pressure under bird exclusion.

Our results show high context dependency of yield determining effects. Such pattern suggests that general conclusions regarding top predator-mesopredator-herbivore-plant food webs even in tropical agroforestry are challenging and far from being fully understood. Although, we could show, that cacao farmers should refrain from disturbing ant communities and maintain $30-40 \%$ shade-tree canopy cover not only for ecophysiological reasons but also to buffer variability in predator communities.

Chapter 3: Resource distribution changes species-specific trophic positions and ecosystem service provision of omnivorous ants.

This chapter aims to determine how ants depend on spatiotemporal distributions of food sources, and how they respond to cacao tree phenology.

We studied changes in $\delta^{15} \mathrm{~N}$ signatures and $\mathrm{C}-\mathrm{N}$ ratios of ant larvae, ant colony size and intensity of prey foraging over time for two Dolichoderinae ants (D. thoracicus and $P$. cf. cordata) in Indonesian cacao agroforestry. We found that density adaptation and trophic plasticity are necessary to compensate spatiotemporal patterns of resource distribution in agricultural landscapes. If food was scarce, both species integrated more honeydew in their diets and colony sizes dropped. Increasing caterpillar abundances resulted in increasing trophic positions and colony sizes. The number of available trophobionts influenced colony sizes only. Amounts of accessible resources did not correlate with ants' hunting effort, which indicates that predatory services of ants are not directly driven by resource availability.

Our result suggests that studying intraspecific trophic plasticity and nutritional stoichiometry helps to identify the ecological role of omnivores and whether a species provides ecosystem services preying on herbivores, or disservices trough cryptic-herbivory and intra-guild predation.

Chapter 4: Multi-scale intensification changes trophic position of omnivores in tropical agroforestry.

This chapter aims to determine how shade cover and distance to forest margins changes the nitrogen and carbon resource-use of omnivorous ants.

We sampled ants from naturally occurring ant communities within 15 plantations along gradients of shade management (canopy cover) and distance to the rainforest margin, to identify and analyse stable isotope ratios of nitrogen $\left(\delta^{15} \mathrm{~N}\right)$ and carbon $\left(\delta^{13} \mathrm{C}\right)$. We found that trophic levels covered by ant communities decreased under high intensification (low shade tree cover and far 
from the forest margins). Trophic positions of particular omnivorous ants show similar patterns (Polyrhachis dives (SMITH, 1857), Camponotus reticulatus (ROGER, 1863), Monomorium floricola (JERDON, 1851) and Technomyrmex albipes (SMITH, 1861), while other species maintained stable tropic positions along both gradients (Anoplolepis gracilipes (SMITH, 1857), Tetramorium pacificum (MAYR, 1870), Odontomachus simillimus (SMITH, 1858). Intraspecific trophic positions - at least partly - influenced the spatial distribution of ant species in cacao agroforestry.

Our results suggest that both, trophic plasticity and species turnover, are mechanisms defining the trophic functions of omnivore communities. Moreover, strong disturbance at local and landscape scales can lower the predatory function of omnivore communities, whereby the magnitude depends on the locally present species. Thus, we infer that detailed ecological knowledge at species level is mandatory to identify the role of omnivores such as ants in ecosystem services provision.

Chapter 5: Intra-specific plasticity in dietary resource-use maintains inter-specific complementarity in natural ant communities.

This chapter aims to reveal whether ant communities show evidence of systematic trophic shifts to reduce interspecific interference, resulting in increased complementarity of ant species.

We sampled ants from naturally occurring ant communities within 15 plantations presenting distinct ant communities. Using the nitrogen and carbon stable isotopic ratios $\left(\delta^{15} \mathrm{~N}\right.$, $\delta^{13} \mathrm{C}$ ) as indicators for trophic position and basal food source, respectively, we test whether the pair-wise distances between values ants taken along these two axes are larger within a community than would be expected given the isotopic ratios of the same species take across communities.

Our results suggest that ant species in a community will exploit resources in a complementary way, most likely to minimize costs related to interspecific interference. The enhanced complementarity due to trophic shifts could mean that ecosystem functioning may be more stable than expected across heterogeneous, highly diverse communities. 


\section{References}

Achard, F., H. D. Eva, H.-J. Stibig, P. Mayaux, J. Gallego, T. Richards, and J.-P. Malingreau. 2002. Determination of Deforestation Rates of the World's Humid Tropical Forests. Science 297:999-1002.

Angelsen, A. 1995. Shifting cultivation and "deforestation": A study from Indonesia. World Development 23:1713-1729.

Armbrecht, I., I. Perfecto, and E. Silverman. 2006. Limitation of nesting resources for ants in Colombian forests and coffee plantations. Ecological Entomology 31:403-410.

Balvanera, P., A. B. Pfisterer, N. Buchmann, J.-S. He, T. Nakashizuka, D. Raffaelli, and B. Schmid. 2006. Quantifying the evidence for biodiversity effects on ecosystem functioning and services. Ecology letters 9:1146-56.

Bos, M. M., J. M. Tylianakis, I. Steffan-Dewenter, and T. Tscharntke. 2008. The invasive Yellow Crazy Ant and the decline of forest ant diversity in Indonesian cacao agroforests. Biological Invasions 10:1399-1409.

Brown, W. L. 2000. Diversity of ants. Pages 45-79 Ants standard methods for measuring and monitoring biodiversity.

Cardinale, B. J., J. E. Duffy, A. Gonzalez, D. U. Hooper, C. Perrings, P. Venail, A. Narwani, G. M. Mace, D. Tilman, D. A. Wardle, A. P. Kinzig, G. C. Daily, M. Loreau, J. B. Grace, A. Larigauderie, D. S. Srivastava, and S. Naeem. 2012. Biodiversity loss and its impact on humanity. Nature 486:59-67.

Clough, Y., D. Dwi Putra, R. Pitopang, and T. Tscharntke. 2009a. Local and landscape factors determine functional bird diversity in Indonesian cacao agroforestry. Biological Conservation 142:1032-1041.

Clough, Y., H. Faust, and T. Tscharntke. 2009b. Cacao cycles, sustainability, and conservation. Conservation Letters 2:197-205.

Costanza, R., R. D’Arge, R. de Groot, S. Farber, M. Grasso, B. Hannon, K. Limburg, S. Naeem, R. V O’Neill, J. Paruelo, R. G. Raskin, P. Sutton, and M. van den Belt. 1997. The value of the world's ecosystem services and natural capital. Nature 387:253-260.

Costanza, R., B. Fisher, K. Mulder, S. Liu, and T. Christopher. 2007. Biodiversity and ecosystem services: A multi-scale empirical study of the relationship between species richness and net primary production. Ecological Economics 61:478-491.

Daily, G. 1997. Nature's Services: Societal Dependence On Natural Ecosystems. Page 412 Corporate Environmental Strategy. Island Press.

Davidson, D. W., S. C. Cook, R. R. Snelling, and T. H. Chua. 2003. Explaining the abundance of ants in lowland tropical rainforest canopies. Science 300:969-972.

Duffy, J. E. 2002. Biodiversity and ecosystem function: the consumer connection. Oikos 99:201219. 
Fischer, J., B. Brosi, G. C. Daily, P. R. Ehrlich, R. Goldman, J. Goldstein, D. B. Lindenmayer, A. D. Manning, H. A. Mooney, L. Pejchar, J. Ranganathan, and H. Tallis. 2008. Should agricultural policies encourage land sparing or wildlife-friendly farming?

Fisher, B., R. K. Turner, and P. Morling. 2009. Defining and classifying ecosystem services for decision making. Ecological Economics 68:643-653.

Floren, A., A. Biun, and E. Linsenmair. 2002. Arboreal ants as key predators in tropical lowland rainforest trees. Oecologia 131:137-144.

Franzen, M., and M. Borgerhoff Mulder. 2007. Ecological, economic and social perspectives on cocoa production worldwide. Biodiversity \& Conservation 16:3835-3849.

Gallai, N., J.-M. Salles, J. Settele, and B. E. Vaissière. 2009. Economic valuation of the vulnerability of world agriculture confronted with pollinator decline. Ecological Economics 68:810-821.

Geist, H. J., and E. F. Lambin. 2002. Proximate Causes and Underlying Driving Forces of Tropical Deforestation. BioScience 52:143.

Germer, J., and J. Sauerborn. 2007. Estimation of the impact of oil palm plantation establishment on greenhouse gas balance. Environment, Development and Sustainability 10:697-716.

Hölldobler, B., and E. O. Wilson. 1990. The Ants. Page 746. Belknap Press of Harvard University Press, Massachusetts.

Hooper, D. U., E. C. Adair, B. J. Cardinale, J. E. K. Byrnes, B. A. Hungate, K. L. Matulich, A. Gonzalez, J. E. Duffy, L. Gamfeldt, and M. I. O’Connor. 2012. A global synthesis reveals biodiversity loss as a major driver of ecosystem change. Nature 000:1-5.

Hunt, J. H. 2003. Cryptic Herbivores of the Rainforest Canopy. Science 300:916-917.

Loreau, M., and A. Hector. 2001. Partitioning selection and complementarity in biodiversity experiments. Nature 412:72-76.

Marín, L., and I. Perfecto. 2013. Spider Diversity in Coffee Agroecosystems: The Influence of Agricultural Intensification and Aggressive Ants. Environmental Entomology 42:204-213.

Martin, E. A., B. Reineking, B. Seo, and I. Steffan-Dewenter. 2013. Natural enemy interactions constrain pest control in complex agricultural landscapes. PNAS 110:5534-5539.

Matson, P. A., W. J. Parton, A. G. Power, and M. J. Swift. 1997. Agricultural Intensification and Ecosystem Properties. Science 277:504-509.

Millennium Ecosystem Assessment. 2003. Ecosystems and Human Well-being: A Framework for Assessment. Pages 1-25 Millennium Ecosystem Assessment. Island Press.

Millennium Ecosystem Assessment. 2005. Ecosystems a human well-being: Sythesis. Island Press, Washington, DC.

Mooney, H. a, A. Cropper, and W. Reid. 2004. The millennium ecosystem assessment: what is it all about? Trends in Ecology \& Evolution 19:221-224. 
Myers, N., R. A. Mittermeier, C. G. Mittermeier, G. A. B. da Fonseca, and J. Kent. 2000. Biodiversity hotspots for conservation priorities. Nature 403:853-858.

Naeem, S., D. E. Bunker, A. Hector, M. Loreau, and C. Perrings. 2009. Biodiversity, Ecosystem Functioning, \& Human Wellbeing. An ecological and economic perspective. OXFORD University Press, Oxford.

Nelson, E., G. Mendoza, J. Regetz, S. Polasky, H. Tallis, D. R. Cameron, K. M. A. Chan, G. C. Daily, J. Goldstein, P. M. Kareiva, E. Lonsdorf, R. Naidoo, T. H. Ricketts, and M. R. Shaw. 2009. Modeling multiple ecosystem services, biodiversity conservation, commodity production, and tradeoffs at landscape scales. Frontiers in Ecology and the Environment 7:411.

Perfecto, I., and J. Vandermeer. 2008. A New Conservation Paradigm. Annals of the New York Academy of Sciences:173-200.

Perfecto, I., J. Vandermeer, P. Hanson, and V. Cartín. 1997. Arthropod biodiversity loss and the transformation of a tropical agro-ecosystem. Biodiversity and Conservation 6:935-945.

Philpott, S. M., and I. Armbrecht. 2006. Biodiversity in tropical agroforests and the ecological role of ants and ant diversity in predatory function. Ecological Entomology 31:369-377.

Philpott, S. M., and P. F. Foster. 2005. Nest-Site Limitation in Coffee Agroecosystems: Artificial Nests Maintain Diversity of Arboreal Ants. Ecological Applications 15:1478-1485.

Power, A. G. 2010. Ecosystem services and agriculture: tradeoffs and synergies. Philosophical Transactions of the Royal Society B 365:2959-2971.

Rice, R. A., and R. Greenberg. 2000. Cacao Cultivation Biological Diversity and the Conservation of Biological Diversity. Ambio 29:167-173.

Robertson, M. M., and J. D. Wainwright. 2013. The Value of Nature to the State. Annals of the Association of American Geographers 103:890-905.

Tilman, D., J. Fargione, L. Authors, F. S. Chapin, R. Dirzo, T. Kitzberger, B. Gemmill, M. Zobel, C. Mitchell, A. Wilby, G. C. Daily, M. Galetti, F. William, J. Pretty, R. Naylor, A. Power, D. Harvell, C. Authors, S. Potts, C. Kremen, T. Griswold, C. Eardley, R. Editors, G. Ceballos, S. Lavorel, G. Orians, S. Pacala, and J. Supriatna. 2009. Biodiversity Regulation of Ecosystem Services. Pages 298-329 in R. Hassan, R. Scholes, and N. Ash, editors. Ecosystem and Human Well-being: Current Sate and Trends. Island Press, Washington, DC.

Tscharntke, T., A. M. Klein, A. Kruess, I. Steffan-Dewenter, and C. Thies. 2005. Landscape perspectives on agricultural intensification and biodiversity - ecosystem service management. Ecology Letters 8:857-874.

Van Bael, S., S. M. Philpott, R. Greenberg, P. Bichier, N. a Barber, K. a Mooney, and D. S. Gruner. 2008. Birds as predators in tropical agroforestry systems. Ecology 89:928-34.

Vandermeer, J., I. Perfecto, G. I. Nuñez, S. Phillpott, and A. G. Ballinas. 2002. Ants (Azteca sp.) as potential biological control agents in shade coffee production in Chiapas, Mexico. Ecology 56:271-276. 
Vandermeer, J., I. Perfecto, and S. Philpott. 2010. Ecological Complexity and Pest Control in Organic Coffee Production: Uncovering an Autonomous Ecosystem Service. BioScience 60:527-537.

Wanger, T. C., D. T. Iskandar, I. Motzke, B. W. Brook, N. S. Sodhi, Y. Clough, and T. Tscharntke. 2010. Effects of Land-Use Change on Community Composition of Tropical Amphibians and Reptiles in Sulawesi, Indonesia. Conservation Biology 24:795-802.

Wenny, D. G., T. L. Devault, M. D. Johnson, D. Kelly, C. H. Sekercioglu, F. Tomback, Diana, and C. J. Whelan. 2011. The Need to Quantify Ecosystem Services Provided By Birds. The Auk 128:1-14.

Whelan, C. J., D. G. Wenny, and R. J. Marquis. 2008. Ecosystem Services provided by Birds. Annals of the New York Academy of Sciences 1134:25-60.

Wielgoss, A., Y. Clough, B. Fiala, A. Rumede, and T. Tscharntke. 2012. A minor pest reduces yield losses by a major pest: plant-mediated herbivore interactions in Indonesian cacao. Journal of Applied Ecology 49:465-473.

Wielgoss, A., T. Tscharntke, D. Buchori, B. Fiala, and Y. Clough. 2010. Temperature and a dominant dolichoderine ant species affect ant diversity in Indonesian cacao plantations. Agriculture, Ecosystems \& Environment 135:253-259.

Wielgoss, A., T. Tscharntke, A. Rumede, B. Fiala, H. Seidel, S. Shahabuddin, and Y. Clough. 2014. Interaction complexity matters: disentangling services and disservices of ant communities driving yield in tropical agroecosystems. Proceedings of the Royal Society B 281:20132144. 


\section{Chapter 2: How ants, birds and bats affect crop yield along shade}

\section{gradients in tropical agroforestry}

Pierre Gras, Teja Tscharntke, Bea Maas, Awal Hafsah, Aijen Tjoa, Yann Clough

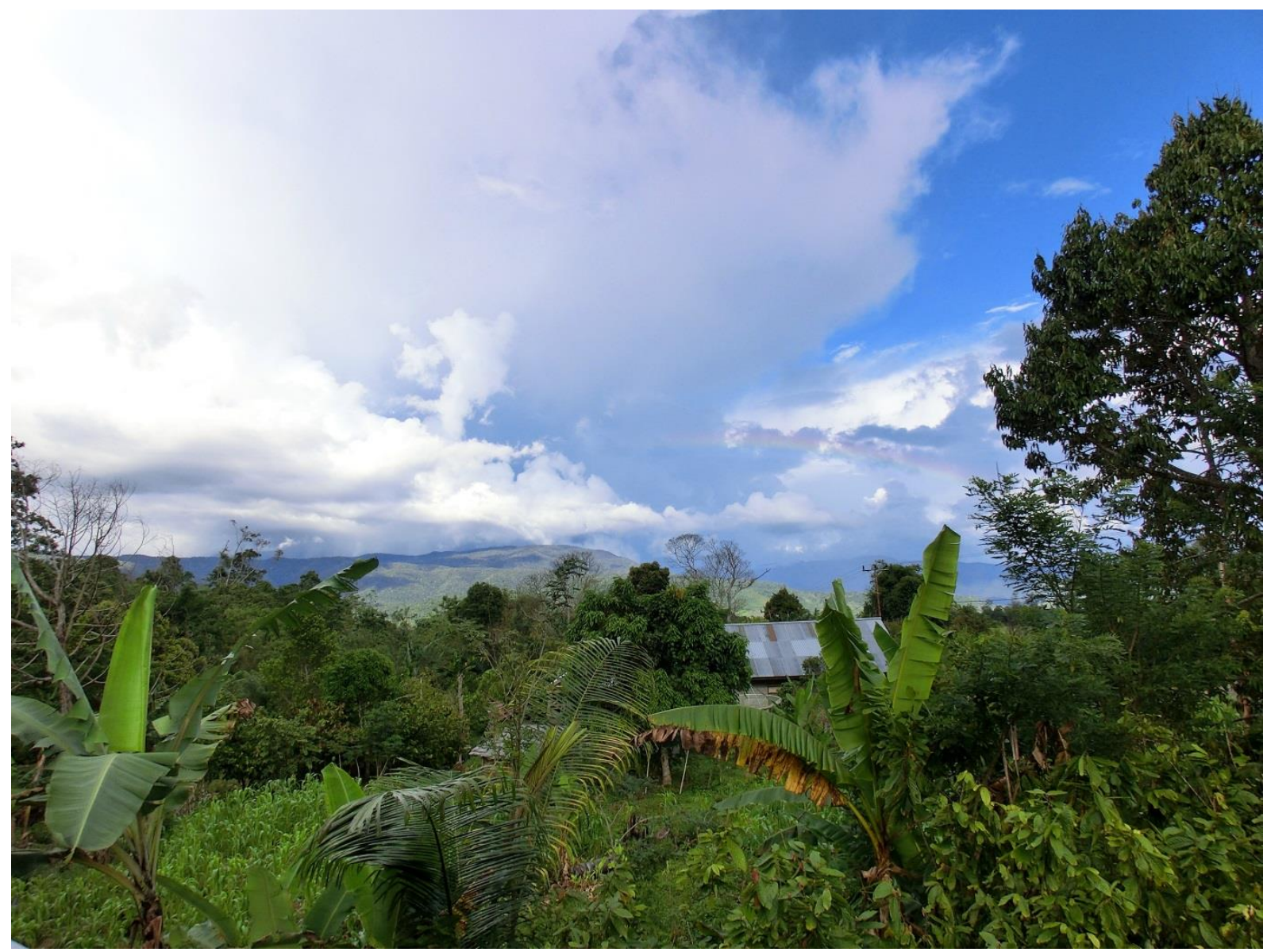

Wuasa is a village in the Napu valley close to the border of the Lore Lindu National Park. The picture shows the vegetation around Wuasa. The vegetation typically consists of forest tree remnants, cacao agroforests, some coffee and fruit trees, vegetable, maize and, paddy fields.

Manuscript submitted to: Journal of Applied Ecology (status: resubmitted minor revision) 


\section{Summary}

1. Tropical agroforests are diverse systems where several predator groups shape animal communities and plant-arthropod interactions. Ants, birds and bats in particular can reduce herbivores and thereby increase crop yield. However, the relative importance of these groups, whether they interact, and how this is affected by management and landscape context, is poorly understood.

2. We jointly manipulated access of ants, birds and bats in Indonesian smallholder cacao agroforestry across gradients of shade and distance to natural forest. We quantified arthropod abundance, pest damage and yield.

3. In control treatments, yield was highest under 30-40\% canopy cover. Ant exclusion strongly reduced yield (from 600 to $300 \mathrm{~kg} \mathrm{ha}^{-1}$ year $^{-1}$ ) at 15\% canopy cover. Bird exclusion impaired yield (from 400 to $250 \mathrm{~kg} \mathrm{ha}^{-1}$ year $^{-1}$ ) at $60 \%$ and enhanced yield (from 600 to $900 \mathrm{~kg} \mathrm{ha}^{-1} \mathrm{year}^{-1}$ ) at $15 \%$ canopy cover, while bats had no effect. Yield increased with forest proximity, a pattern not related to predator access.

4. No interactive effects among predator exclusions on yield, pest damage and arthropod communities were found. Ant exclusion increased numbers of herbivores below 30\% canopy cover, without reducing spider abundances. Bird exclusion reduced herbivore and increased spider abundances.

5. Synthesis and applications. The impact of ants and birds on cacao yield is economically relevant, and depends on shade-tree management. In all but the most shaded agroforests, ants were pivotal in supporting yields. Yields under lower canopy cover were strongly dependent on access by predator groups. Hence, cacao farmers should refrain from disturbing ant communities and maintain 30-40\% shade-tree canopy cover not only for ecophysiological reasons but also to buffer variability in predator communities. 


\section{Introduction}

Predators exert top-down control and can positively or negatively influence plant development through direct and indirect interactions affecting pests and diseases (Vandermeer $e t$ al. 2002; Vandermeer, Perfecto \& Philpott 2010). Detailed reviews covering effects of ants, birds and bats on arthropod communities and crop yield, often assessed using exclusion experiments, underline their importance for ecosystem service provision (Mooney et al. 2010, Philpott \& Armbrecht 2006; Whelan, Wenny \& Marquis 2008; Van Bael et al. 2008; Wenny et al. 2011; Kunz et al. 2011; Maas et al. 2015b).

Ants are effective biological control agents, especially in tropical agroforestry. For example, in Mexican coffee arboreal ants protect trees from colonisation by important pests (Gonthier et al. 2013). However, impacts of ants depend on the environmental context (e.g. temperature), ant species involved (Philpott \& Armbrecht 2006; Gove 2007; Wielgoss et al. 2014), potential counterproductive effects on mesopredators such as spiders (Eubanks 2001), mutualism, plantssucking trophobionts (i.e. Aphids and mealbugs) that are harmful to crops. Birds have also been shown to reduce abundances of pests (Holmes, Schultz \& Nothnagle 1979; Johnson et al. 2009), with yield losses up to $\$ 310 \mathrm{ha}^{-1}$ year $^{-1}$ prevented in coffee agroforestry, for example (Karp et al. 2013). Effects of birds on arthropod communities are not always that strong, can negatively affect mesopredators, and do not necessarily trickle down to plants (Maas, Clough \& Tscharntke 2013, Williams-Guillén, Perfecto \& Vandermeer 2008). Bats have received increased attention as biocontrol agents in recent years following several seminal reports from natural forests and cacao agroforests (Williams-Guillén, Perfecto \& Vandermeer 2008, Kalka, Smith \& Kalko 2008). Efforts to disentangle bird and bat effects have partly confirmed the potential of bats as biocontrol agents in agroforestry (Maas, Clough \& Tscharntke 2013, Maas et al. 2015b), and partly suggested that negative impacts on spiders can cancel out the effects that bats may have on herbivores (Karp \& Daily 2014).

Effects of these predator groups have usually been considered in isolation, using exclusion experiments conducted in only one or a handful of locations. In reality, density and diversity of predators, and thus probably also their biological control effect, depend on local management or landscape context (Clough et al. 2009, Karp et al. 2013). In addition, it is unclear whether impacts of the three predator groups are complementary or redundant. Few studies have tested interactive effects (but see Mestre et al. 2013b, Mooney 2007, Piñol et al. 2010, Philpott et al. 2004, Spiller \& Schoener 2001). Finally, few studies quantify impacts on crop yield, making economic assessments difficult since plants can often compensate for damage (but see Mooney et al. 2010; Wielgoss et al. 2014, Maas, Clough \& Tscharntke 2013; Karp \& Daily 2014). 
Here, we simultaneously investigate effects and management dependency of ant, bird and bat exclusions in 15 smallholder cacao plantations differing in \%-shade-tree canopy cover and distance to forest margins. In our study region Central Sulawesi (Indonesia) results from separate experiments revealed that both ant and combined bird-bat exclusions decreased yield by $\sim 30 \%$ (Maas, Clough \& Tscharntke 2013, Wielgoss et al. 2014). These effects may change along canopy cover gradients as local shade-tree management impacts productivity, fruit abortion, and arthropod and vertebrate communities (Bos, Steffan-Dewenter \& Tscharntke 2007a, Clough et al. 2009). Increasing distance from natural forest can also negatively affect the density and diversity of bird and bat communities, and therefore the degree of biological control (Clough et al. 2009, Estrada \& Coates-Estrada 2002). In the present study we addressed the following questions:

(1) Does exclusion of ants, birds and bats affect cacao yield, and if so, does this change with shade-tree canopy cover or distance to forest margin?

(2) Are effects of the three manipulated predator groups synergistic, additive or antagonistic?

(3) What are the likely processes explaining impacts of ant, bird, or bat exclusions on cacao crop yield, pest and disease infestation, and fruit set or fruit abortion?

(4) What are the management implications for cacao farmers in terms of canopy cover, landscape-scale forest preservation and predator conservation?

\section{Material and methods}

\section{Site selection and gradients}

Fifteen smallholder Theobroma cacao L. plantations were selected at the eastern border of the Lore Lindu National Park in Central Sulawesi, Indonesia (Fig. 1; 1²3'31.8"S, 120¹8'57.55"E, $1130 \mathrm{~m}$ a.s.l., 1990-3804 mm rainfall) to cover five distances to forest margin (Fig. S1, three agroforests each category: 0-250 m, 251-500 m, 501-1500 m, 1501-2500 m, 2501-3000 m) and three canopy cover types (Fig. S1, five agroforests each category: $<30 \%, 30-50 \%,>50 \%$ ). Later, both variables were measured in meters to forest margin or \%-shade-tree canopy cover. The latter was measured at the experimental treatment level (within-agroforest), but variability was larger between than within agroforests. Local farmers managed all agroforests and we incorporated pesticide, herbicide and fungicide free areas to minimize management differences. Nonetheless, each agroforest provided a unique habitat due to agroforest size, cacao tree age/abundance, previous/nearby land-use, topography, drainage, fertilizer-use (Table S18) or intercropped vegetables and fruits (Tables S2-3). The agroforests were separated by at least $500 \mathrm{~m}$ edge-to-edge distance. Two planted legume shade-tree species - Erythrina subumbrans Merr. and Gliricidia sepium 
Kunth - represented 30 - 91\% of all recorded non-crop trees (Table S2, herb species in Table S3). We trimmed the herb layers every two months using a motor scythe. In the course of the experiment we recorded 49 ant and 69 bird species (Tables S4-6).

\section{Exclusion treatments}

Bird and bat exclusions were implemented in 2010 (cf. Maas, Clough \& Tscharntke 2013), and ant exclusions were added in April 2011, both were maintained until June 2012. Exclusions consisted of eight randomly positioned treatments (two trees each) per study site: (1) no exclusion, (2) ant-exclusion, (3) bird-exclusion, (4) bat-exclusion, (5) ant-bird-exclusion, (6) ant-bat-exclusion, (7) bird-bat-exclusion and (8) ant-bird-bat-exclusion.

We used bamboo scaffolds covered with fishing nets ( $35 \times 35 \mathrm{~mm}$ mesh size) to exclude birds and/or bats. The bird-and-bat exclusion treatments had fixed nets. Bird exclusion nets were manually opened in the morning (5:30 am) and closed in the evening $(6: 30 \mathrm{pm})$, while bat exclusions were opened in the evening $(6: 30 \mathrm{pm})$ and closed in the morning $(5: 30 \mathrm{am})$. Controls were always open. Open nets (top and all sides) were bound tightly to the scaffolds (Fig. S7). To exclude ants, we used cone-shaped insect glue rings fitted to the tree trunks (Fig. S8), thereby reducing contact between ring and trunk and preventing trunks from getting mouldy - a problem encountered while planning a previous study (Wielgoss et al. 2014). Thus, ant exclusion effects are similar to conventional insect glue applications. We destroyed ant nests during establishment of ant exclusions, whenever discovered and during a monthly "search-and-destroy"-survey. Arthropod exchange through net-tree contact was avoided by regularly pruning braches to keep at least $30 \mathrm{~cm}$ between foliage and nets/scaffolds. Effectiveness of ant exclusion was quantified by counting ant individuals from canopy knock-down fogging samples; showing that ant abundance was strongly reduced (by $60-90 \%$, see results) in exclusions compared to control treatments.

\section{Sampling and surveying methods}

We obtained distances to forest margins and agroforest area using a Garmin Oregon 550 Global Positioning System device (Fig. 1). We took treatment-level digital hemispherical canopy photos and calculated \%-canopy cover using the CIMES-FISHEYE software (Walter 2009). We measured several potentially yield-related covariates: (1) cacao tree crown volume and (2) mean DBH to represent tree size, (3) daily mean temperature and (4) precipitation to represent abiotic conditions, (5) non-crop tree richness known to correlate with the bird community composition (Clough et al. 2009), (6) the average branch perimeter as indicator of fruit carrying capacity, and (7) the pod groove depth which indicates 'on-fruit' hiding opportunities for arthropods and can differ between agroforests depending on the cacao phenotype (Tables S9 \& S10). 
Every two weeks from April 2011 to May 2012, we surveyed all experimental trees counting and classifying cacao fruits by size/development and presence/absence of pest and disease symptoms and harvesting of ripe fruits (classification details, Tables S11 \& S12). We separately quantified the weight of marketable and damaged beans (i.e. due to the damage of the cacao pod borer (Conopomorpha cramerella)). Damage caused by the most economically important organisms (1) C. cramerella, (2) Helopeltis sulawesi and (3) Phytophthora palmivora was assessed by counting fruit with damage symptoms.
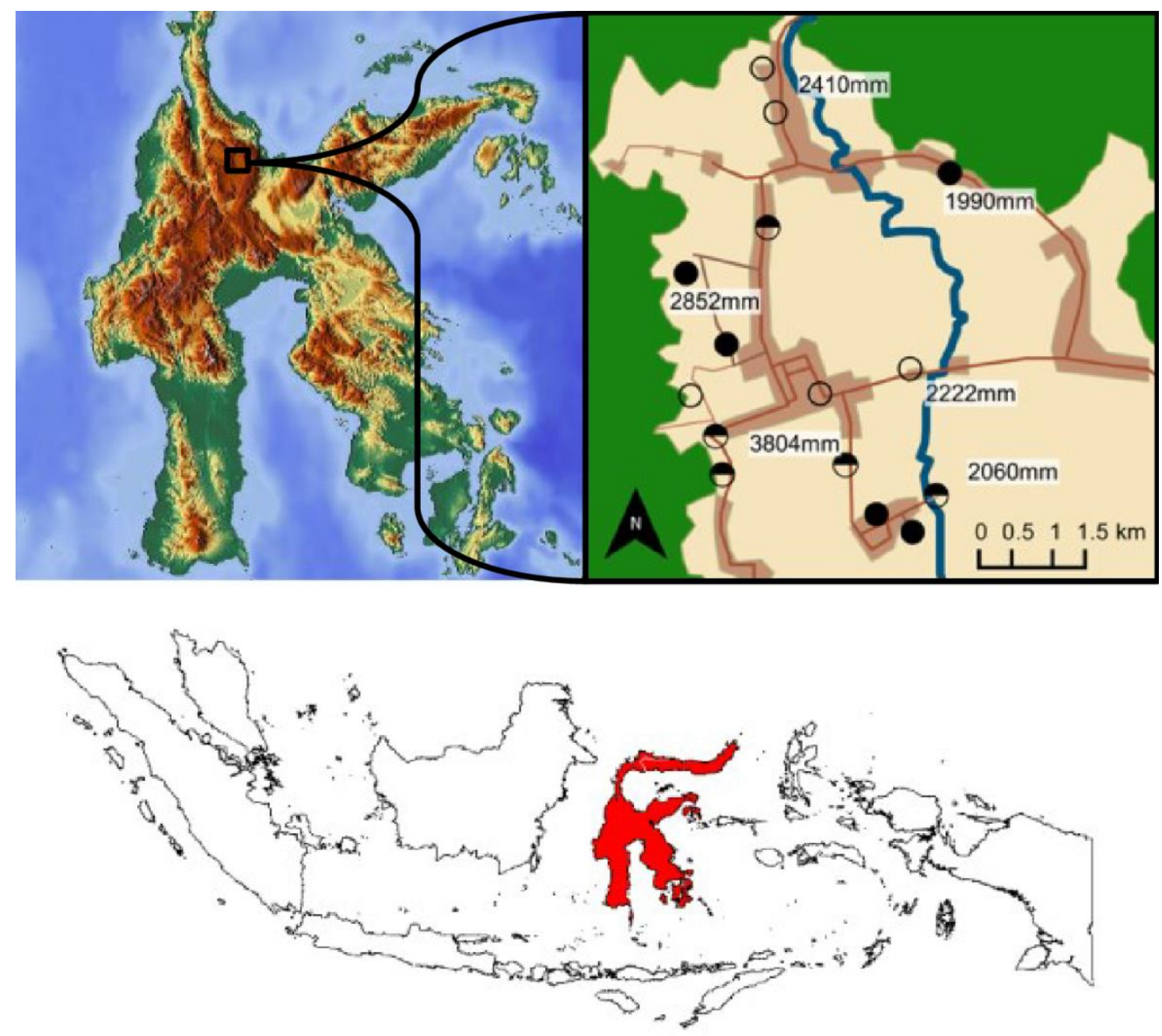

Fig. 1. Study area in the Napu valley of Central Sulawesi, Indonesia. Bottom: Map of Indonesia red area indicates Sulawesi. Top-left: Topography of Sulawesi and location of the study area (black square). Top-right: Cycles show experimental cacao agroforests, filling style indicates canopy cover (empty $<30 \%$, half $=30-50 \%$, filled $>50 \%$ ), white labels show rainfall in $\mathrm{mm}$, green area indicates rainforest, reddish areas houses, bright areas open land and lines streets.

We recorded other pests damaging leaves, flowers, and fruits (e.g. aphids, herbivorous bugs and caterpillars), as well as mesopredators (e.g. spiders, earwigs, and lacewing larvae) through 
canopy knock-down fogging with a mixture of $5 \%$ Malathion (Fumithion 1150 ULV) and diesel fuel (Bos, Steffan-Dewenter \& Tscharntke 2007b, Rizali et al. 2013) at the end of the experiment. We fogged all treatments (covered by plastic tents) for 5 minutes and left the tents closed for one hour. Fogging samples were used to test predator exclusion effects on arthropod orders.

Every four weeks (from May 2011 to March 2012), ants were baited on trees without ant exclusions to detect effects of shade/ forest distance on ant community abundance, richness and composition. We used protein (tin-tuna in brine) and carbohydrate (saturated sugar solution) baits. We identified ant/morpho-species using taxonomic literature (Bolton 1994; Fisher 2010) and regional ant collections (Wielgoss et al. 2014; Rizali et al. 2013).

Between September 2010 and June 2011, the bird community was recorded by repeated mist netting surveys and point count recordings on all 15 study sites (see Maas et al. 2015a for details).

\section{Statistical analyses}

Data from 28 harvests were summed for each agroforest and treatment. Response variables directly related to yield (total marketable yield, fruit abundance, and fruit weight), were analysed in three steps. First, we fitted a full model containing the random factor "experimental agroforest", and the terms of interest: (1) three binary exclusion variables and their interaction terms, (2) agroforest-level design variables (canopy cover, forest distance) and (3) their interaction with each exclusion treatment variable, (4) seven covariates: crown volume, mean $\mathrm{DBH}$, temperature, precipitation, non-crop tree richness, branch perimeter and pod groove depth. A second-order polynomial term was included for canopy cover, to allow for non-linearity (Tscharntke et al. 2011). Continuous explanatory variables were z-transformed. We fitted the model using maximum likelihood and Gaussian (marketable yield, yield per fruit, number of harvested fruit, leaf area, leaf damage), overdispersion-corrected Poisson (arthropod abundances), or binomial (proportions of infested fruits) distribution. For Gaussian models root- or log-transformed response variable were used to meet assumptions of homoscedasticity and (approximate) normality of the residuals. Models were assessed with variance inflation factors and diagnostic plots for residual normality, heteroscedasticity and leverage.

Second, retaining all variables of interest, the model was simplified by keeping those covariates that contributed to the model, as indicated by a multi-model inference procedure ("MuMIn" R-package, AIC based). The tree crown volume differed between treatments and negatively correlated with canopy cover $(\mathrm{F}=-2.092, P=0.04)$. Therefore, we included crown volume as permanent covariate during all statistical analyses. Third, the same procedure was used to identify non-significant interactions that were removed for model simplification and refitted a 
model using restricted maximum likelihood. In the results section, the variables of interest and their interactions were reported when relative variable importance values (proportion of models including the variable vs. models excluding the variable out of the best set of models, identified by delta AIC <2) exceeded 0.9 for main effect interactions and 0.7 for covariates. We used the lme4 package in R (Bates et al. 2014; R Core Team 2014) and reported statistical significance from the final model using ANOVA Type II, Wald Chi ${ }^{2}$ test (Kuznetsova, Brockhoff \& Christensen 2014).

To determine the mechanisms leading to differences in marketable yields, only predictors identified as important for marketable yield (see S13) were used on the following responses: (1) open flowers, (2) aborted small fruits, (3) leaf herbivory. To test patterns of herbivorous arthropod abundances, incidence of pests and disease symptoms, and beneficial arthropod abundances we used: (1) fruit infestation of H. sulawesi and C. cramerella, (2) Sternorrhyncha, (3) caterpillars $>10$ mm, (4) Coleoptera >10 mm, (5) Auchenorrhyncha, (6) Dermaptera, (7) Neuroptera larvae, (8) hunting-spiders $<4 \mathrm{~mm}$, (9) hunting-spiders 4-10 mm, (10) web spiders $<4 \mathrm{~mm}$, (11) web spiders 4-10 mm, (12) ants and (13) Diptera $<4 \mathrm{~mm}$.

Finally, changes in community composition were tested using species richness, diversity, evenness and composition of ants and birds along the gradients of canopy cover and forest distance. The measurements were rarefied to 737 individuals per site for ants and 122 individuals per site for birds. We conducted a redundancy analyses and visualized community composition using Minimum Convex Polygons (MCP) at the level of the agroforest (birds) and experimental treatment (ants), and, finally, tested for the influences of canopy cover, forest distance and treatment using a permutation test $(\mathrm{n}=999)$. Separate analyses were conducted for each variable, with both remaining variables and crown volume being set as conditions. Crown volume and treatment were not included for bird community analyses, as birds were recorded at the agroforest level.

\section{Results}

\section{Effects of predator exclusion, canopy cover and distance to forest on cacao yield}

Yields of control trees varied between $75 \%$ and $135 \%$ of the average productivity per hectare of $540 \mathrm{~kg}^{-1}$ year ${ }^{-1}$ for Indonesia in 2012 (Fig. 2, S13.1; yield Indonesia 2012: http:/ / faostat3.fao.org). In control trees without exclusions, marketable yield, proportion of fruits without pest infestation and the number of harvested fruits peaked under $30-40 \%$ shade-tree canopy cover (Figs. 2 \& 3G-L, S13.1-3). The yield was affected by ant and bird exclusion with the magnitude and direction of the effect depending on shade-tree canopy cover. Yield was not affected by bat exclusion (Fig. 2, S13.1). Interactions between exclusion treatments were not 
significant. Independently of predator exclusions, marketable yields and numbers of harvested fruits decreased with increasing forest distance (S13.1-3).

\section{Ant exclusion}

Ant exclusions reduced ant abundances by $60-90 \%$ (Fig. 5A; S13.11). Marketable yield, the number of harvested fruit and fruit weight were reduced under ant exclusion (Figs 2, 3J \& 3M; S13.1-3). Ant abundances were positively correlated with marketable yields while controlling for exclusion treatments $\left(\mathrm{X}^{2}=7.22, \mathrm{P}<0.01\right)$.

Below 30\% canopy cover, ant exclusions were associated with fewer flowers, and a trend towards fewer aborted small fruits compared to control treatments (Figs 3A \& 3D; S13.4-5). Ant exclusion did not affect pest and disease incidents at fruits (Fig. 3G; S13.6). Canopy cover above $50 \%$ reversed the differences in aborted small fruits, with higher numbers in ant exclusions than in controls (Fig. 3D). Losses of flowers and small fruits were reflected in reduced numbers of harvested fruits under ant exclusion (Fig. 3J; S13.3). Additionally, fruit weight was reduced in the absence of ants (Fig. 3M; S13.2).

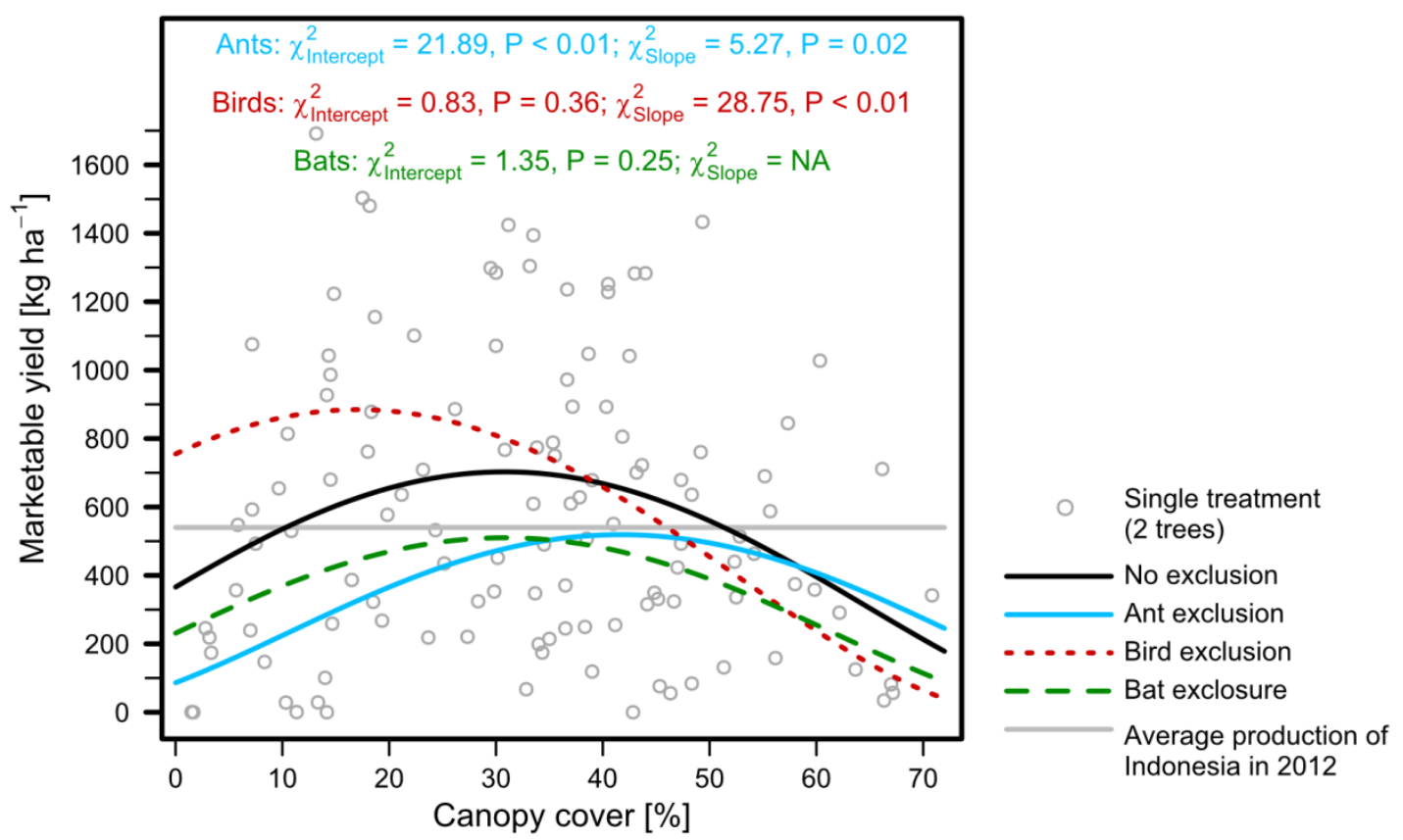

Fig. 2. Effects of predator exclusion on marketable cacao bean yield. Dry yield (y-axis) of exclusion treatments (control $=$ continuous-black, no ants $=$ blue $/$ grey, no birds $=$ dotted-red, no bats $=$ dashed-green $)$ dependent on $\%$ canopy cover (x-axis) in Indonesian cacao agroforests, thin-red line marks the average cacao production of Indonesia 2012 (http:/ / faostat3.fao.org), yield was standardised to 12 month for plotting but not for analyses. $\mathrm{X}^{2}$ Intercept indicates influences of predator exclusions, while $\mathrm{X}^{2}$ Slope indicates canopy cover dependency of predator exclusions. 
Abundances of lepidopteran caterpillars $(>10 \mathrm{~mm})$ and beetles significantly increased in response to ant exclusions while abundances of caterpillars $(0-4 \mathrm{~mm})$ and leafhoppers remained unaffected (Figs 4A-J; S13.7-10). Leaf damage was increased in ant exclusions below 30\% canopy cover $\left(\mathrm{X}^{2}=6.54, P=0.01 ; \mathrm{S} 13.16\right)$.

Spiders were largely unaffected by ant exclusion, with only a trend towards small webbuilding spiders being more frequent in ant exclusions below 30\% canopy cover. (Figs 5D \& 5G; S13.12-13). Below 30\% canopy cover, earwigs were absent, but when present, their abundance was reduced in ant exclusions (Fig. 5J; S13.14). Small beetles were more abundant under ant exclusion and with above $50 \%$ canopy cover (Fig. 5M; S13.15).

\section{Bird exclusion}

Compared to controls, marketable cacao yields were slightly reduced in bird exclusions when canopy cover was above 50\%. Canopy cover below 30\% lead to higher yields in bird exclusions $\left(500 \mathrm{~kg} \mathrm{ha}^{-1}\right.$ to $830 \mathrm{~kg} \mathrm{ha}^{-1}$ year $^{-1}$, Fig. 2; S13.1). Comparable patterns were found for flowers, aborted small fruits, fruits without damage by pests such as $H$. sulawesi and C. cramerella, and harvested fruits (Figs 3B-H; S13.4-7). Amount of non-infected and harvested fruits were significantly correlated $\left(\mathrm{Chi}^{2}=32.87, \mathrm{P}<0.01\right)$. The fruit weight remained unaffected by bird exclusion (Fig. 3N; S13.3).

Caterpillar and beetle abundances remained unaffected by bird exclusion, but abundances of Auchenorrhyncha were reduced (Figs. 4B-K, S13.7-10). Simultaneously, web-building spider abundance increased, although this effect was only significant for large individuals $(4-10 \mathrm{~mm})$, where it was stronger over 50\% canopy cover (Fig. 5H, S13.13). Increases in small web spiders $(<4 \mathrm{~mm}$ ) were marginally significant, and tended to be higher with canopy cover less $30 \%$ (Fig. 5E, S13.12). Small beetles $(<4 \mathrm{~mm})$ tended to be less abundant with more than $30 \%$ canopy cover (Fig. $5 \mathrm{~N}, \mathrm{~S} 13.15)$. Earwigs had lower densities due to bird exclusion, an effect restricted to canopy cover higher 40\% (Fig. 5K, S13.14). Ants were not significantly affected by bird exclusion (Fig. 5B, S13.11). Yield effects of bird exclusion persisted when adding ant abundance as model covariate. Small dipterans were less frequent under bird exclusions $\left(\mathrm{X}^{2}\right.$ Bird exclusion $=6.59, P=0.01$; S15.17).

\section{Bat exclusion}

Excluding bats had no significant effect on marketable yield. No effect could be detected on flowers, aborted small fruits, fruit infestation by pests and diseases, harvested fruits or fruit weight (Fig. 2, 3C-O; S15.1-6). Bat exclusion was associated with higher numbers of leaf hoppers and, for canopy cover over 50\%, higher numbers of large caterpillars (4-10mm) (Fig 4F \& 4L, S15.7 \& S15.10). Small caterpillars or beetles remained unaffected (Fig. 4A \& 4I, S15.7 \& S15.9). Bat 
exclusion did not affect mesopredators smaller four millimetre body length, but was associated with higher spider and earwig abundance (Fig. 5C-O, S15.11-15).

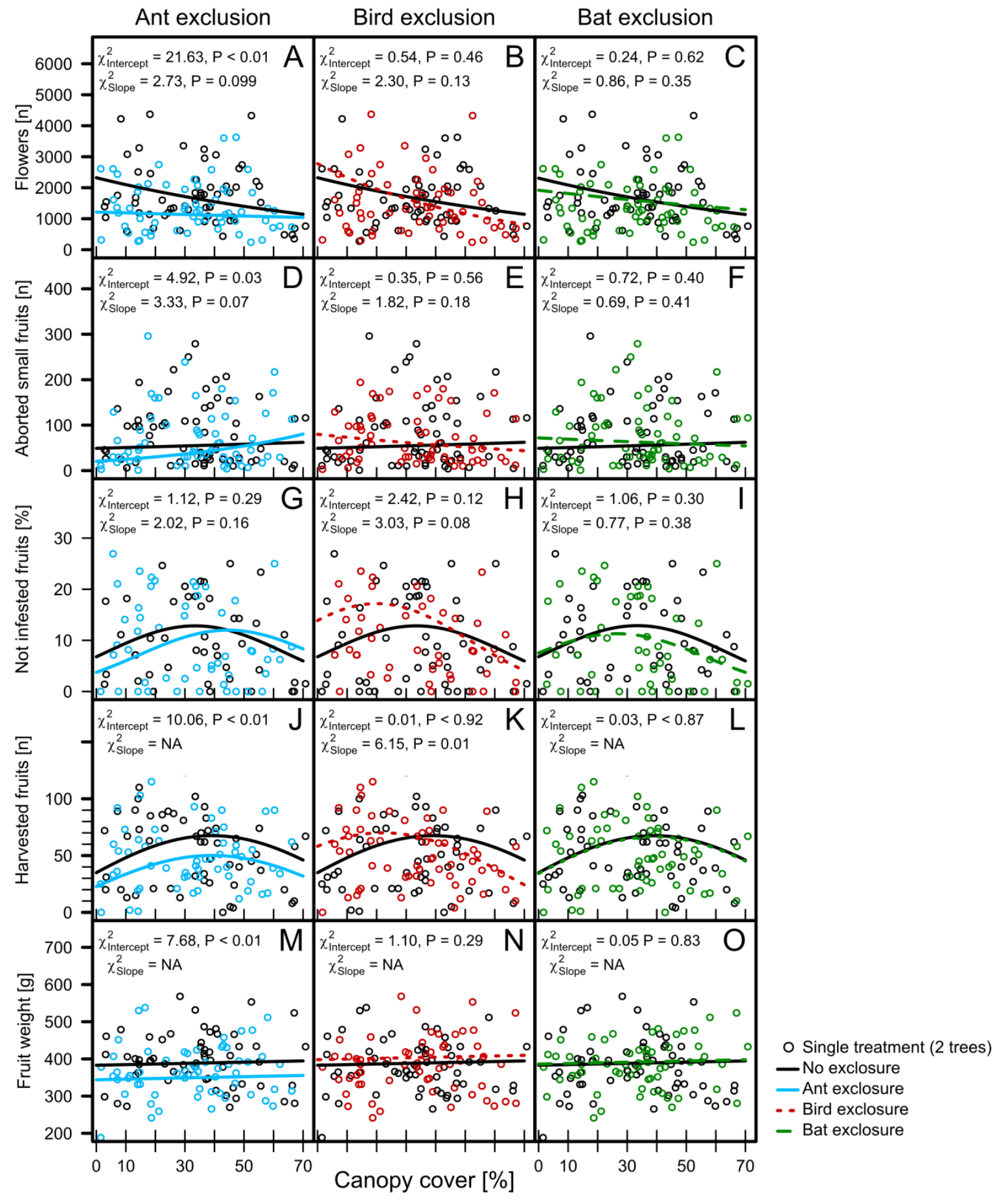

Fig. 3. Effects of predator exclusion on cacao tree phenology. Small figures shows the response of cacao trees to ant (left), bird (mid) or bat (right) exclusion related to canopy cover (x-axis), coloured circles correspond to predator of each small figure, line types: control $=$ continuous-black, no ants $=$ blue/grey, no birds $=$ dotted-red, no bats $=$ dashed-green. $\mathrm{X}^{2}$ Intercept indicates influences of predator exclusions, while $\mathrm{X}^{2}$ slope indicates canopy cover dependency of predator exclusions. 


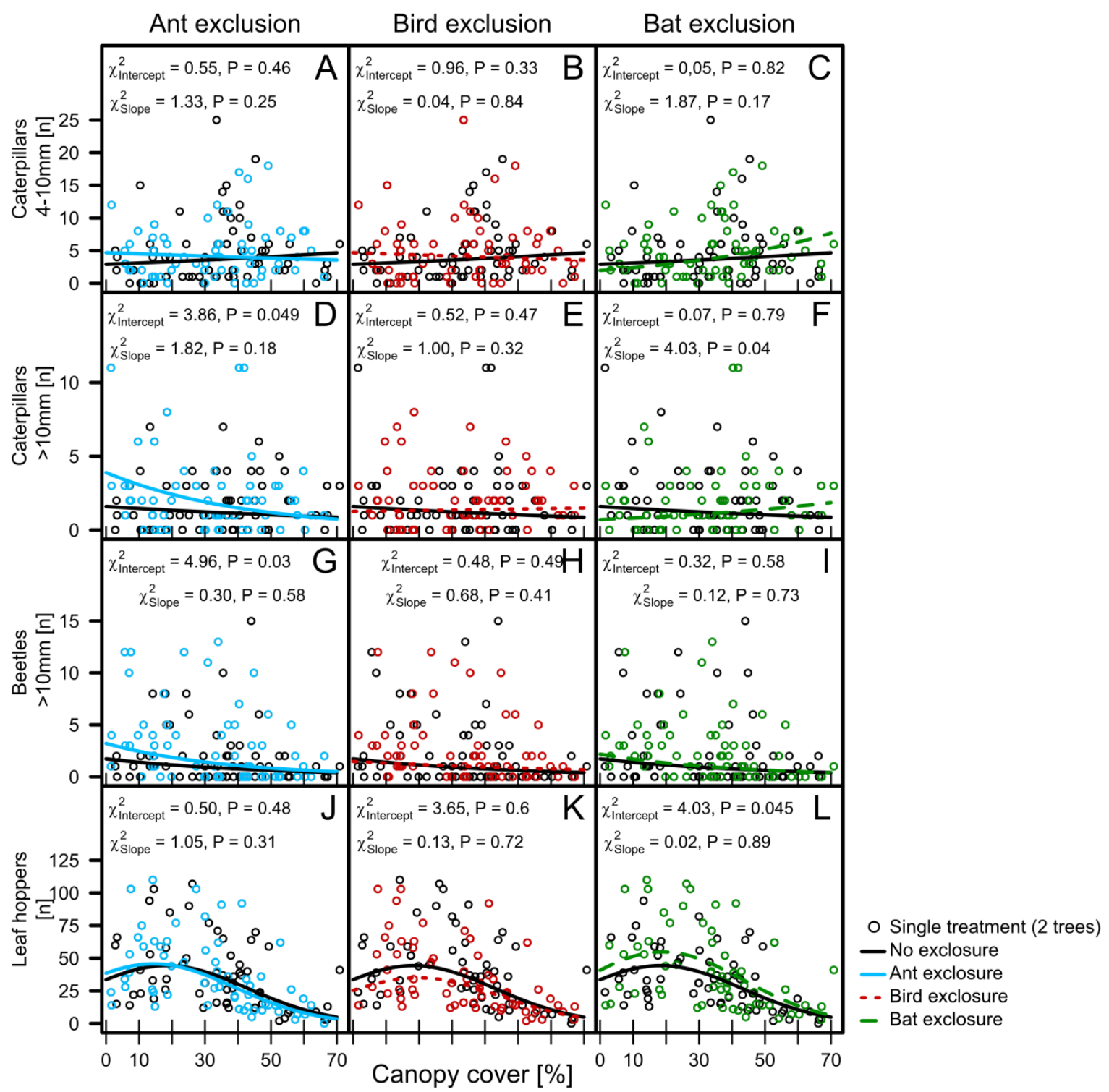

Fig. 4. Effects of predator exclusion on cacao tree herbivores. Small figures shows the response of herbivores to ant (left), bird (mid) or bat (right) exclusion related to canopy cover ( $\mathrm{x}$-axis), coloured circles correspond to predator of each small figure, line types: control = continuous-black, no ants $=$ blue/grey, no birds $=$ dotted-red, no bats $=$ dashed-green, $\mathrm{X}^{2}$ Intercept indicates influences of predator exclusions, while $\mathrm{X}^{2}$ slope indicates canopy cover dependency of predator exclusions.

\section{Ant and bird diversity along the canopy cover and forest distance gradient}

Ant species composition (RDA, Fig. S14) was similar across those treatments where ants were present $(\mathrm{F}=0.76, P=0.84)$, but ant species composition strongly changed with \%-canopy $\operatorname{cover}(\mathrm{F}=2.81, P=0.001)$ and forest distance $(\mathrm{F}=2.93, P=0.001)$. Species composition of the local bird assemblage (RDA, Fig. S15) changed due to forest distance ( $F=1.31, P=0.03)$, but did not respond to canopy cover $(F=1.12, P=0.33)$. Shannon index, species evenness and rarefied species richness of ants and birds where independent of canopy cover and forest distance (S16). 


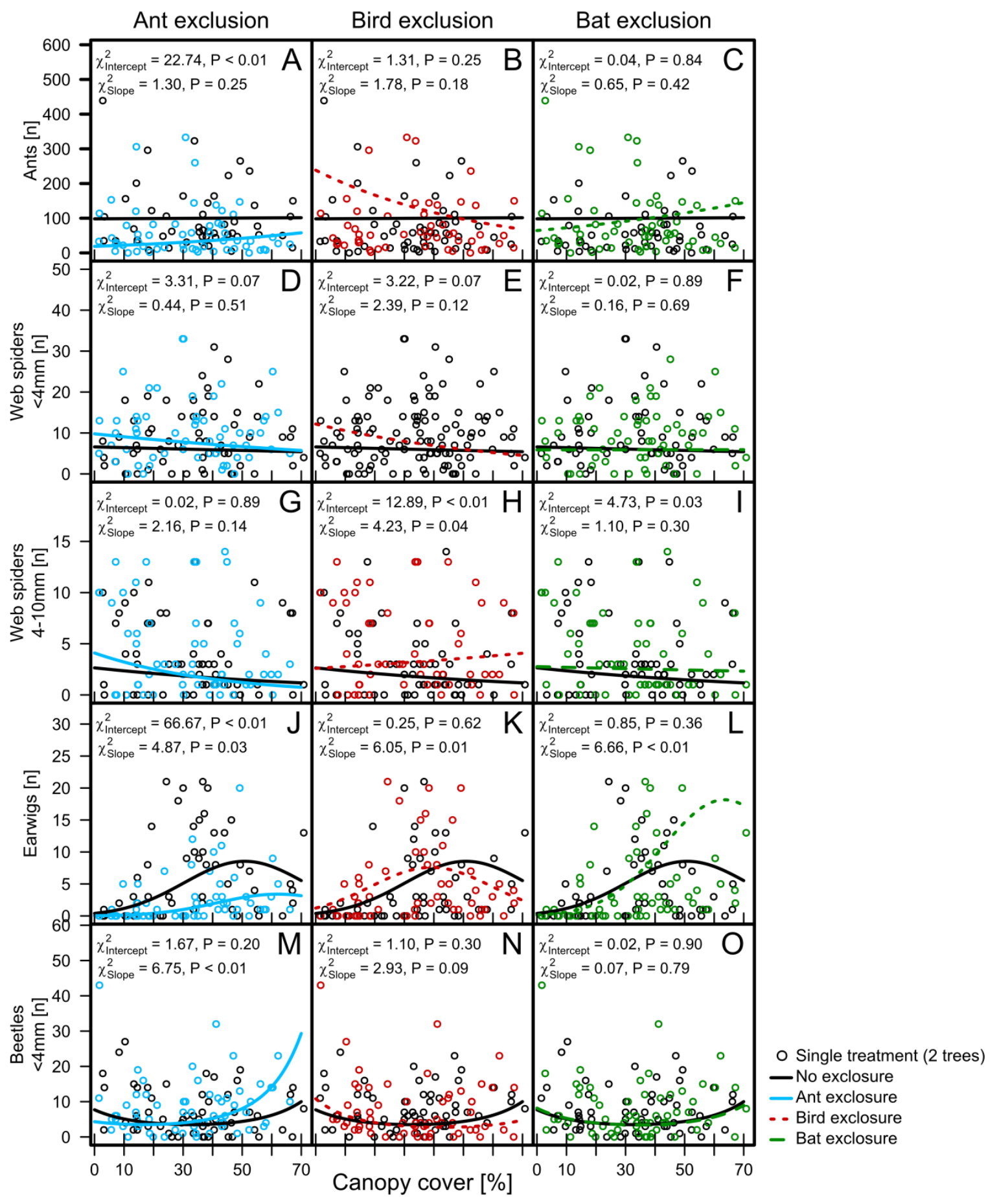

Fig. 5. Effects of predator exclusion on cacao tree mesopredators. Small figures shows the response of mesopredators to ant (left), bird (mid) or bat (right) exclusion related to canopy cover (x-axis), coloured circles correspond to predator of each small figure, line types: control $=$ continuous-black, no ants $=$ blue $/$ grey, no birds $=$ dotted-red, no bats $=$ dashed-green, $\mathrm{X}^{2}$ Intercept indicates influences of predator exclusions, while $\mathrm{X}^{2}$ Slope indicates canopy cover dependency of predator exclusions. 


\section{Discussion}

Access by predators, \%-shade-tree canopy cover and distance to forest margin had economically relevant impacts on cacao yield. While the negative effect of forest distance was independent of predator exclusion, \%-canopy cover affected the impact of predators. Yield peaked at $30-40 \%$ canopy cover. Canopy cover below $15 \%$ or above $55 \%$ resulted in less than half of the optimum yield. Ant exclusion reduced yields, at 15\% canopy cover from 600 to $300 \mathrm{~kg} \mathrm{ha}^{-1}$ year $^{-1}$. Bird exclusion decreased yields at $60 \%$ canopy cover from 400 to $250 \mathrm{~kg} \mathrm{ha}^{-1} \mathrm{year}^{-1}$. Unexpectedly, bird exclusion increased yields at $15 \%$ canopy cover from 600 to $900 \mathrm{~kg} \mathrm{ha}^{-1} \mathrm{year}^{-1}$. Bats had no effect on yield.

Canopy cover dependent effects of ant and bird exclusions in agroforests have never been shown previously, despite several recent studies targeting these groups in tropical agroforestry (Wielgoss et al. 2014, Maas et al. 2015b). Our results can partly be explained by patterns in yield formation, as well as impacts on herbivorous and predatory arthropods that were quantified by fogging the experimental trees at the end of the study period.

\section{Canopy-cover dependent effects of ants}

Ants affect plants by tending/defending or predating/displacing herbivores, but also predating/displacing predators (such as spiders) and pollinators, spreading propagules of plant pathogens (Wielgoss et al. 2014), pollinating flowers, predating/dispersing seeds, and changing soil conditions (Bartlett 1961; Way 1963; Mestre et al. 2013b, 2014). Their numeric and ecological dominance in tropical agroforestry systems likely affects trees in multiple ways (e.g. plant growth, leaf and fruit development). In our study, impacts of ant exclusion have their likely cause in the interaction of arboreal ants and other arboreal organisms such as herbivores: ants were excluded from trees and not the ground below, tent-building species able to spread pathogens were absent, and ants are not able to pollinate cacao (Leston 1970). Interference between ants and spiders has been reported from tree crops (Piñol, Espadaler \& Cañellas 2012; Mestre et al. 2012; Mestre, Bucher \& Entling 2014 but see Marín \& Perfecto 2013). Here, and in contrast to what was observed in response to bird exclusion, no significant change in spider abundance occurred under ant exclusion, suggesting that ants do not limit the abundance of spiders. Instead, ants reduced abundances of herbivores such as caterpillars and beetles that damage not only leaves but also cacao flowers (YC personal observation; Bos, Steffan-Dewenter \& Tscharntke 2007a; Maas, Clough \& Tscharntke). Increased herbivore abundance, parallel increased leaf damage, and fewer flowers under ant exclusion probability resulted in fewer fruits. Simultaneously, reinforcing negative effects on yield, fruit weight was reduced when ants were absent, which is associated with infestation by fruitdamaging pests such as $H$. sulawesi and C. cramerella (Wielgoss et al. 2014). Impacts of ants on crop 
yield, including their economic importance, are similar to those reported from sites situated in the same region at lower altitudes (Wielgoss et al. 2014), but our results demonstrate that ant predation effects were only economically relevant for agroforests providing less than $40 \%$ canopy cover. Generally, low-shade conditions can be more stressful for cacao trees, both in terms of ecophysiology, and in terms of herbivory (De Almeida \& Valle 2007; Tscharntke et al. 2011). These findings are in accordance with our results, showing that small beetles and caterpillar densities increased under lower shade-tree canopy cover. Besides this, we could show that there was significant ant species turnover across the gradient in canopy cover. It thus cannot be excluded that ant species more dominant under low-canopy cover conditions provide a more effective biological control of pests than species dominant under higher canopy cover.

\section{Bird exclusions can decrease or increase yields depending on canopy cover}

Against expectation, excluding birds led to more fruits and higher proportions of healthy fruit below 30\% canopy cover. Lowered or similar yields under bird exclusion would have been expected throughout the canopy cover gradient, but were observed only in relation to canopy cover above $40 \%$. We assume that the effects of excluding birds are caused by the absence of insectivorous birds, of which 31 species were recorded at cacao canopy level (Maas et al. 2015a). Indeed, none of the bird species recorded feed on cacao fruits. Woodpecker damage can occasionally be seen on fruit (YC personal observation), but is likely to only affect fruit with prior pest infestation. Pollination reduction due bird exclusion is unlikely, since cacao flowers are very small and pollinated by midges, not birds (Leston 1970). The bird community composition did not change with canopy cover, suggesting that shade-dependency of yields under bird exclusions may be due to observed differences in arthropod pest and mesopredator densities along the gradient. Generally, there was little response in herbivore abundance to bird exclusion. Leafhopper numbers were depressed and earwig numbers promoted under bird exclusion when shade tree canopy cover was below 30\%. This, together with lower numbers of damaged fruits under bird exclusion, points to indirect positive effects on herbivores by birds (e.g. mesopredator predation) under little shaded conditions where herbivory affects cacao trees more severely (Tscharntke et al. 2011). The exact mechanism is unclear, as the fogging data shows only a towards increases in predator densities at canopy cover below 30\% for ants and small web spiders with increases and decreases at canopy cover above 50\% for larger web spiders and earwigs, respectively. Generally, increased abundances of predators, such as ants, spiders and earwigs, under bird exclusions, are common (Gunnarsson 2007; Williams-Guillén , Perfecto \& Vandermeer 2008; Piñol et al. 2010; Mestre et al. 2013a; Maas, Clough \& Tscharntke 2013). Overall, increases in most predatory arthropods and decreases in individual herbivores due to bird exclusion suggest mesopredator release and may at least partly 
explain the ambivalent effects of birds on cacao yield in our study, although food-web data will be necessary to confirm this. The impact of lower abundances in small Dipterans $(<4 \mathrm{~mm})$, which includes cacao-pollinating Ceratopogonidae (Leston 1970) is unknown, yet could conceivable reduce pollination and thereby cause yield losses (Groeneveld et al. 2010).

\section{Bats}

In 2010, at least 16 insectivorous bat species were recorded in agricultural areas around the Lore Lindu National Park in two separate studies by Graf and Boonman (unpublished data, see S17). Several of the species glean insects from leaves or perch on plants to prey on arthropods associated with plants, and might therefore be relevant to our experimental exclusions. However, bat exclusion did not affect yield, or yield-related plant variables, but enhanced abundances of leafhoppers, large caterpillars, and earwigs and large spiders, i.e. both herbivorous and predatory arthropods. Bats have strong effects on arthropod communities in tropical forests and agricultural systems (Williams-Guillén, Perfecto \& Vandermeer 2008; Kalka, Smith \& Kalko 2008; Wanger et al. 2014). In coffee, bats can promote herbivorous arthropods by reducing spider densities, and as a result, do not necessarily provide biocontrol (Karp \& Daily 2014). A previous study from cacao in our study region, however, showed bats were instrumental in decreasing pests (Maas, Clough \& Tscharntke 2013). This suggests that the impact of bats could be strongly dependent on the net balance of arthropod herbivores and predators, with bat impacts on both groups cancelling out any top-down effect on the plants in the present study. Leaf-gleaning bats are less abundant in agricultural systems than in natural forest (Phommexay et al. 2011) suggesting natural forests are sources of bats for nearby agroforests, but we did not find any evidence for forest distance dependent effects of bats.

\section{Study limitations and caveats}

The extent to which we can causally underpin the detected significant effects is limited by the study design in some respects. First, the impact of forest distance on yield, while significant, cannot be fully elucidated, as it seems not to involve the manipulated predators. Secondly, while we tried to control for variables that could not be standardized, unwanted exclusion effects may have occurred. For instance, the reduction of earwig abundances under ant exclusion may have been due to the ant exclusion rings partly excluding crawling earwigs. While this may have led to an overestimation of the impact of ants under high canopy cover, impacts of ants were strongest below 30\% canopy cover, where earwigs were always scarce, so our conclusions on ant effects are robust. Moreover, earwig abundances that increased over 40\% canopy cover in bat exclusions did not affect cacao yield. Thirdly, we cannot exclude that the presence of nets deterred certain birds 
or bats from foraging inside the agroforests, due to the risk of collision. Finally, the drawback of a long study duration was the impossibility of adequately surveying arthropods during the whole course of the experiment. Parallels between herbivores and predators recorded through fogging, and cacao tree variables recorded over the whole study duration, allow us to draw hypotheses on mechanisms, but not to formally test them.

\section{Management recommendations}

Impacts of ants and birds on cacao yield are economically relevant, but depend on shadetree management. In all but most shaded agroforests, ants were pivotal in supporting yields, reducing populations of herbivorous insects rather than other beneficial organisms such as spiders. This confirms the importance of ants for the economic performance of cacao agroforestry in Central Sulawesi. Farmers should be aware of changes in ant communities due to shifts in management or invasion by other species, and avoid destroying ant nests, as it is often done for weaver ants in the region, for example (YC \& PG personal observation). Moreover, current cacao farming methods entail self-shaded or low-shaded ( $10 \%$ shade-tree canopy cover) plantations impairing arthropod and plant diversity (Rice \& Greenberg 2000). The observation that, on average, shade-tree canopy cover of $30-40 \%$ yielded most, suggests that farmers should maintain such intermediate canopy cover levels not only for ecophysiological reasons (Tscharntke et al. 2011) but also to buffer variability in predator communities. Our data suggests that beneficial effects of forest proximity on yield were unlikely to be related to pest limitation services by ants and birds. However, positive effects of forest proximity on predation of dummy caterpillars, mediated by higher abundances of a locally common white-eye species (Zosterops chloris), has been demonstrated in our study area (Maas et al. 2015a), and together these results suggest that farmers should have an interest in the stability of forest margins, which is threatened by encroachment.

\section{Acknowledgements}

We are grateful to partners at Tadulako University Palu, IPB Bogor and LIPI. We thank Arjan Boonman and Stefan Graf for providing bat survey data of our study region, Akhmad Rizali (ants), Hardianto Mangopo (trees) and Firdaus (herbs) for specimen identifications and Arno Wielgoss, Iris Motzke, Ilfianty Kasmundin and Pak Abdul Rauf for support. Special thanks we address to all research (Dadang Dwi-Putra, Edi Djismin, Alfianus Rumede), lab (Ivon, Abe and Fatma), and field assistants (Opu, Nimus, Anto, Tia, Papa Ifer, Idi, Soni, Wandi, Anki, Sardin and

Sony), all supportive farmers and project members, especially Wolfram Lorenz. The project was funded by DFG (ELUC CL-474/1-1 and CRC 990 EFForTS). 


\section{References}

Bartlett, B.R. (1961) The influence of ants upon parasites, predators, and scale insects. Annals of the Entomological Society of America, 54, 543-551.

Bates, D., Maechler, M., Bolker, B. \& Walker, S. (2014) lme4: linear mixed-effects models using S4 classes. R package version 1.1-6. $R$.

Bolton, B. (1994) Identification Guide to the Ant Genera of the World. Harvard University Press, Cambridge.

Bos, M.M., Steffan-Dewenter, I. \& Tscharntke, T. (2007a) Shade tree management affects fruit abortion, insect pests and pathogens of cacao. Agriculture, Ecosystems \& Environment, 120, 201-205.

Bos, M.M., Steffan-Dewenter, I., Tscharntke, T. (2007b) The contribution of cacao agroforests to the conservation of lower canopy ant and beetle diversity in Indonesia. Biodiversity and Conservation, 16, 2429-2444.

Clough, Y., Dwi-Putra, D., Pitopang, R. \& Tscharntke, T. (2009) Local and landscape factors determine functional bird diversity in Indonesian cacao agroforestry. Biological Conservation, 142, 1032-1041.

De Almeida, A.-A.F. \& Valle, R.R. (2007) Ecophysiology of the cacao tree. Brazilian Journal Of Plant Physiology, 19, 425-448.

Estrada, A., \& Coates-Estrada, R. (2002) Bats in continuous forest, forest fragments and in an agricultural mosaic habitat-island at Los Tuxtlas, Mexico. Biological Conservation, 103, 237-245.

Eubanks, M.D. (2001) Estimates of the direct and indirect effects of red imported fire ants on biological control in field crops. Biological Control, 21, 35-43.

Fisher, B.L. (2010) Ants of Borneo - Guide to the Ant Genera of Borneo (Ant course 2010).

Gonthier, D.J., Ennis, K.K., Philpott, S.M., Vandermeer, J. \& Perfecto, I. (2013) Ants defend coffee from berry borer colonization. BioControl, 58, 815-820.

Gove, A.D. (2007) Ant biodiversity and the predatory function (A response to Philpott and Armbrecht, 2006). Ecological Entomology, 32, 435-436.

Groeneveld, J.H., Tscharntke, T., Moser, G. \& Clough, Y. (2010) Experimental evidence for stronger cacao yield limitation by pollination than by plant resources. Perspectives in Plant Ecology, Evolution and Systematics, 12, 183-191.

Gunnarsson, B. (2007) Bird Predation On Spiders: Ecological Mechanisms And Evolutionary Consequences. Journal of Arachnology, 35, 509-529. 
Holmes, R.T., Schultz, J.C. \& Nothnagle, P. (1979) Bird predation on forest insects: an exclosure experiment. Science, 206, 462-463.

Johnson, M.D., Levy, N.J., Kellermann, J.L. \& Robinson, D.E. (2009) Effects of shade and bird exclusion on arthropods and leaf damage on coffee farms in Jamaica's Blue Mountains. Agroforestry Systems, 76, 139-148.

Kalka, M.B., Smith, A.R. \& Kalko, E.K.V. (2008) Bats limit arthropods and herbivory in a tropical forest. Science, 320, 71.

Karp, D.S. \& Daily, G.C. (2014) Cascading effects of insectivorous birds and bats in tropical coffee plantations. Ecology, 95, 1065-1074.

Karp, D.S., Mendenhall, C.D., Sandí, R.F., Chaumont, N., Ehrlich, P.R., Hadly, E.A. \& Daily, G.C. (2013) Forest bolsters bird abundance, pest control and coffee yield. Ecology letters, 16, 1339-1347.

Kunz, T.H., de Torrez, E.B., Bauer, D., Lobova, T. \& Fleming, T.H. (2011) Ecosystem services provided by bats. Annals of the New York. Academy of Sciences, 1223, 1-38.

Kuznetsova, A., Brockhoff, P.B. \& Christensen, H.B. (2014) lmer'Test: Tests for random and fixed effects for linear mixed effect models (lmer objects of lme4 package). $R$ package version, $\mathrm{R}$ package version 2.0-6.

Leston, D. (1970). Entomology of the cocoa farm. Annual Review of Entomology, 15, 273-294.

Maas, B., Clough, Y. \& Tscharntke, T. (2013) Bats and birds increase crop yield in tropical agroforestry landscapes. Ecology Letters, 16, 1480-1487.

Maas, B., Tscharntke, T., Saleh, S., Dwi-Putra, D. \& Clough, Y. (2015a) Avian species identity drives predation success in tropical cacao agroforestry. Journal of Applied Ecology, 52, 735-743.

Maas, B., Karp, D.S., Bumrungsri, S., Darras, K., Gonthier, D., Huang, J.C.C., et al. (2015b). Bird and bat predation services in tropical forests and agroforestry landscapes. Biological Reviews. DOI: 10.1111/brv.12211

Marín, L. \& Perfecto, I. (2013) Spider Diversity in Coffee Agroecosystems: The Influence of Agricultural Intensification and Aggressive Ants. Environmental Entomology, 42, 204 213.

Mestre, L., Bucher, R. \& Entling, M.H. (2014) Trait-mediated effects between predators: ant chemical cues induce spider dispersal. Journal of Zoology, 293, 119-125.

Mestre, L., Garcia, N., Barrientos, J.A., Espadaler, X. \& Piñol, J. (2013a) Bird predation affects diurnal and nocturnal web-building spiders in a Mediterranean citrus grove. Acta Oecologica, 47, 74-80. 
Mestre, L., Piñol, J., Barrientos, J.A., Cama, A. \& Espadaler, X. (2012) Effects of ant competition and bird predation on the spider assemblage of a citrus grove. Basic and Applied Ecology, 13, 355-362.

Mestre, L., Piñol, J., Barrientos, J.A. \& Espadaler, X. (2013b) Ant exclusion in citrus over an 8-year period reveals a pervasive yet changing effect of ants on a Mediterranean spider assemblage. Oecologia, 173, 239-248.

Mooney, K.A. (2007) Tritrophic effects of birds and ants on a canopy food web, tree growth, and phytochemistry. Ecology, 88, 2005-14.

Mooney, K.A., Gruner, D.S., Barber, N.A., Van Bael, S.A., Philpott, S.M. \& Greenberg, R. (2010) Interactions among predators and the cascading effects of vertebrate insectivores on arthropod communities and plants. PNAS, 107, 7335-40.

Philpott, S.M. \& Armbrecht, I. (2006) Biodiversity in tropical agroforests and the ecological role of ants and ant diversity in predatory function. Ecological Entomology, 31, 369-377.

Philpott, S.M., Greenberg, R., Bichier, P. \& Perfecto, I. (2004) Impacts of major predators on tropical agroforest arthropods: comparisons within and across taxa. Oecologia, 140, 140-149.

Phommexay, P., Satasook, C., Bates, P., Pearch, M., \& Bumrungsri, S. (2011). The impact of rubber plantations on the diversity and activity of understorey insectivorous bats in southern Thailand. Biodiversity and Conservation, 20, 1441-1456.

Piñol, J., Espadaler, X., Cañellas, N., Martínez-Vilalta, J., Barrientos, J.A. \& Sol, D. (2010) Ant versus bird exclusion effects on the arthropod assemblage of an organic citrus grove. Ecological Entomology, 35, 367-376.

Piñol, J., Espadaler, X. \& Cañellas N. (2012) Eight years of ant-exclusion from citrus canopies: effects on the arthropod assemblage and on fruit yield. Agricultural and Forest Entomology, 14, 49-57.

R Core Team. (2014) R: A language and environment for statistical computing. R Foundation for Statistical Computing, Vienna, Austria, URL http:/ /www.R-project.org/.

Rice, R.A. \& Greenberg R., (2000) Cacao Cultivation and the Conservation of Biological Diversity. Ambio, 29, 167-173.

Rizali, A., Clough, Y., Buchori, D., Hosang, M.L.A., Bos, M.M. \& Tscharntke, T. (2013) Long-term change of ant community structure in cacao agroforestry landscapes in Indonesia. Insect Conservation and Diversity, 6, 328-338.

Spiller, D.A., Schoener, T.W. (2001). An experimental test for predator-mediated interactions among spider species. Ecology 82, 1560-1570.

Tscharntke, T., Clough, Y., Bhagwat, S.A., Buchori, D., Faust, H., Hertel, D., Hölscher, D., Juhrbandt, J., Kessler, M., Perfecto, I., Scherber, C., Schroth, G., Veldkamp, E. \& 
Wanger, T. C. (2011) Multifunctional shade-tree management in tropical agroforestry landscapes-a review. Journal of Applied Ecology, 48, 619-629.

Van Bael, S.A., Philpott, S.M., Greenberg, R., Bichier, P., Barber, N.A., Mooney, K.A. \& Gruner, D.S. (2008) Birds as predators in tropical agroforestry systems. Ecology, 89, 928-34.

Vandermeer, J., Perfecto, I., Nuñez, G.I., Phillpott, S. \& Ballinas, A.G. (2002) Ants (Azteca sp.) as potential biological control agents in shade coffee production in Chiapas, Mexico. Ecology, 56, 271-276.

Vandermeer, J., Perfecto, I. \& Philpott, S. (2010) Ecological complexity and pest control in organic coffee production: uncovering an autonomous ecosystem service. BioScience, $60,527-537$.

Walter, J.-M. (2009) CIMES-FISHEYE. Hemispherical Photography of Forest Canopies.

Wanger, T.C., Darras, K., Bumrungsri, S., Tscharntke, T. \& Klein, A.-M. (2014) Bat pest control contributes to food security in Thailand. Biological Conservation, 171, 220-223.

Way, M.J. (1963) Mutualism Between Ants and Honeydew-Producing Homoptera. Annual Review of Entomology, 8, 307-344.

Wenny, D.G., Devault, T.L., Johnson, M.D., Kelly, D., Sekercioglu, C.H., Tomback, Diana, F. \& Whelan, C.J. (2011) The Need to Quantify Ecosystem Services Provided By Birds. The Auk, 128, 1-14.

Whelan, C.J., Wenny, D.G. \& Marquis, R.J. (2008) Ecosystem Services provided by Birds. Annals of the New York Academy of Sciences, 1134, 25-60.

Wielgoss, A., Tscharntke, T., Rumede, A., Fiala, B., Seidel, H., Shahabuddin, S. \& Clough, Y. (2014) Interaction complexity matters: disentangling services and disservices of ant communities driving yield in tropical agroecosystems. Proceedings of the Royal Society B, 281, 20132144.

Williams-Guillén, K., Perfecto, I. \& Vandermeer, J. (2008) Bats limit insects in a neotropical agroforestry system. Science, 320, 70. 
Supporting information - Chapter 2

$45 / 168$ 


\section{Fig. S1. Schematic experimental design.}

One box stands for one experimental site (cacao plantation). Each header names a site. The letter-number code indicates predator exclusion types as specified in the legend. Positions of the exclusion treatments were randomised within on site. Columns indicate distances to the forest margin. Rows show the estimated shade tree canopy cover of each site during site selection.

\begin{tabular}{|c|c|c|c|c|c|c|}
\hline \multirow{13}{*}{ 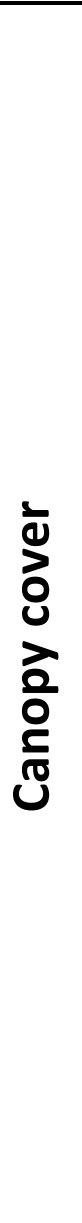 } & & \multicolumn{5}{|c|}{ Distance to forest margin } \\
\hline & & $0-250 m$ & $251-500 m$ & $501-1500 m$ & $1501-2500 m$ & 2501-3000m \\
\hline & \multirow{3}{*}{$0-30 \%$} & Febi & Jem & Ciko & Robi & Dedi \\
\hline & & $A B C D$ & F C E D & A F C D & A B C D & $A B C D$ \\
\hline & & E F G H & $A G H B$ & B E G H & G E F H & $\mathrm{E} \mathrm{GF} \mathrm{H}$ \\
\hline & & Banti & Dewa & Kiki & Toni & Main \\
\hline & & $B A C D$ & $\mathrm{FAGH}$ & $A B G D$ & $\mathrm{~F} \mathrm{E} \mathrm{C} \mathrm{H}$ & $A B C D$ \\
\hline & $31-50 \%$ & E F G H & $E B D C$ & E C F H & $A B G D$ & E F G H \\
\hline & \multirow{5}{*}{$51-80 \%$} & Ahmad & Bolai & Limba & Deni & Ponedi \\
\hline & & $B E C D$ & $A B C D$ & $\mathrm{FACH}$ & $B A C D$ & F G E H \\
\hline & & $\mathrm{F} \mathrm{G} \mathrm{A} \mathrm{H}$ & E F G H & G E B D & E G F H & $B$ A C D \\
\hline & & $A=$ No bats & $B=$ No birds & $C=$ No exclusion & \multicolumn{2}{|c|}{$D=$ No vertebrates } \\
\hline & & $E=$ No bats \& ants & $\mathrm{F}=$ No birds $\&$ ants & $\mathrm{G}=$ No ants & \multicolumn{2}{|c|}{$\mathrm{H}=$ No bats $\&$ birds $\&$ ants } \\
\hline
\end{tabular}


Table S2. Non-crop tree species within chemical application free area.

Names indicate the plantation identity. Column one and two show the taxonomic classification (Family, Species), columns 3 - 17 the site-specific species abundances and column 18 the sum of all sites. (4 pages)

\begin{tabular}{|c|c|c|c|c|c|c|c|c|c|c|c|c|c|c|c|c|}
\hline Family & Species & 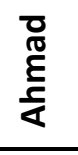 & 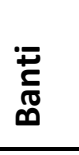 & 而 & 总 & $\begin{array}{l}\overline{\bar{\nu}} \\
\text { هั }\end{array}$ & 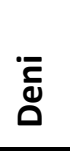 & 蛋 & 矛 & $\stackrel{\varepsilon}{\varrho}$ & 咅 & 疍 & 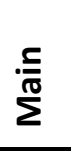 & $\begin{array}{l}\text { ¿̄ं } \\
\bar{\Xi} \\
\overline{0}\end{array}$ & $\begin{array}{l}\text { oे } \\
\text { वे }\end{array}$ & 흠 \\
\hline Anacardiaceae & Mangifera indica $\mathrm{L}$. & 2 & 1 & 1 & 0 & 0 & 0 & 0 & 0 & 3 & 0 & 0 & 0 & 0 & 1 & 0 \\
\hline Annonaceae & Annona muricata $\mathrm{L}$. & 0 & 1 & 0 & 0 & 0 & 0 & 0 & 0 & 0 & 0 & 0 & 0 & 0 & 0 & 0 \\
\hline Annonaceae & Cananga odorata (Lam.) Hook.f. \& Thomson & 0 & 0 & 2 & 0 & 0 & 0 & 0 & 0 & 0 & 0 & 0 & 0 & 0 & 0 & 0 \\
\hline Arecaceae & Arenga pinnata Merr. & 0 & 0 & 5 & 0 & 0 & 0 & 3 & 1 & 6 & 0 & 0 & 1 & 0 & 0 & 0 \\
\hline Arecaceae & Cocos nucifera $\mathrm{L}$. & 0 & 0 & 0 & 3 & 0 & 0 & 0 & 0 & 0 & 0 & 0 & 0 & 0 & 0 & 0 \\
\hline Arecaceae & Pigafetta filaris (Giseke) Becc. & 0 & 0 & 0 & 0 & 0 & 0 & 0 & 1 & 0 & 0 & 0 & 0 & 0 & 0 & 0 \\
\hline Asteraceae & Vernonia arborea Buch.-Ham. & 0 & 0 & 0 & 0 & 0 & 0 & 0 & 0 & 0 & 1 & 0 & 0 & 0 & 0 & 0 \\
\hline Bombacaceae & Ceiba pentandra (L.) Gaertn. & 0 & 0 & 1 & 0 & 0 & 0 & 0 & 0 & 0 & 0 & 0 & 0 & 0 & 0 & 0 \\
\hline Coniferae & Agathis dammara (Lamb.) Rich. & 0 & 1 & 1 & 0 & 0 & 0 & 0 & 0 & 0 & 0 & 0 & 0 & 0 & 0 & 0 \\
\hline Dracaenaceae & Dracaena angustifolia Roxb. & 0 & 0 & 0 & 0 & 0 & 0 & 0 & 1 & 0 & 0 & 0 & 0 & 0 & 0 & 0 \\
\hline Euphorbiaceae & Acalypha caturus Blume & 0 & 0 & 0 & 0 & 0 & 0 & 2 & 4 & 0 & 0 & 0 & 0 & 0 & 0 & 0 \\
\hline Euphorbiaceae & Aleurites moluccanus (L.) Willd. & 1 & 7 & 5 & 0 & 0 & 0 & 3 & 11 & 3 & 0 & 3 & 0 & 0 & 1 & 0 \\
\hline
\end{tabular}


Euphorbiaceae Bischofia javanica Blume.

Euphorbiaceae Glochidion rubrum Blume

Euphorbiaceae Glochidion spp.

Euphorbiaceae Homalanthus populneus Kuntze

Euphorbiaceae Macaranga hispida Müll. Arg.

Euphorbiaceae Macaranga Thouars sp.

Euphorbiaceae Mallotus Lour. Sp.

Euphorbiaceae Xemiti aleuritis

$\infty$

Euphorbiaceae Jatropha curcas L.

Lamiaceae Gmelina arborea Roxb.

Бे Lamiaceae Tectona grandis L.f.

Lauraceae Litsea Lam. Spp.

Lauraceae

Leguminosae

Persea americana Mill.

Leguminosae

Erythrina subumbrans Merr.

Tamarindus indica L.

Meliaceae

cf. Aglaia Lour. Spp.

Metiaceae

Lansium domesticum Jack

Moraceae

Arthocapus sp.2

Moraceae

Artocarpus heterophyllus Lam.

\begin{tabular}{rrrrrrrrrrrrrrr|r}
0 & 0 & 1 & 1 & 1 & 0 & 3 & 6 & 2 & 0 & 0 & 0 & 0 & 0 & 0 & 14 \\
0 & 0 & 0 & 0 & 0 & 0 & 1 & 0 & 0 & 0 & 0 & 0 & 0 & 0 & 0 & 1 \\
0 & 0 & 0 & 0 & 0 & 0 & 0 & 8 & 0 & 0 & 0 & 0 & 0 & 0 & 0 & 8 \\
0 & 0 & 0 & 0 & 0 & 0 & 0 & 2 & 0 & 0 & 1 & 0 & 0 & 0 & 0 & 3 \\
0 & 0 & 0 & 0 & 0 & 0 & 0 & 4 & 0 & 0 & 0 & 0 & 0 & 0 & 0 & 4 \\
0 & 0 & 0 & 0 & 0 & 0 & 7 & 31 & 0 & 0 & 0 & 0 & 0 & 0 & 0 & 38 \\
0 & 0 & 1 & 0 & 0 & 0 & 0 & 0 & 1 & 0 & 1 & 0 & 0 & 0 & 0 & 3 \\
0 & 0 & 0 & 0 & 0 & 0 & 0 & 1 & 0 & 0 & 0 & 0 & 0 & 0 & 0 & 1 \\
0 & 0 & 0 & 0 & 0 & 0 & 0 & 0 & 4 & 0 & 0 & 0 & 0 & 0 & 0 & 4 \\
0 & 1 & 0 & 0 & 0 & 0 & 0 & 0 & 0 & 10 & 0 & 0 & 0 & 3 & 7 & 21 \\
0 & 0 & 0 & 7 & 0 & 0 & 0 & 1 & 0 & 7 & 0 & 0 & 0 & 0 & 0 & 15 \\
0 & 0 & 1 & 0 & 0 & 0 & 0 & 1 & 0 & 0 & 0 & 0 & 0 & 0 & 0 & 2 \\
13 & 0 & 1 & 4 & 0 & 0 & 0 & 9 & 27 & 0 & 6 & 2 & 0 & 4 & 3 & 69 \\
5 & 0 & 15 & 4 & 0 & 0 & 120 & 29 & 36 & 37 & 0 & 33 & 10 & 41 & 1 & 331 \\
29 & 60 & 4 & 23 & 57 & 53 & 60 & 60 & 37 & 6 & 59 & 60 & 55 & 39 & 31 & 633 \\
0 & 0 & 0 & 0 & 0 & 0 & 0 & 0 & 0 & 2 & 0 & 0 & 0 & 0 & 0 & 2 \\
0 & 0 & 0 & 0 & 0 & 0 & 0 & 2 & 0 & 0 & 0 & 0 & 0 & 0 & 0 & 2 \\
0 & 1 & 0 & 1 & 0 & 0 & 0 & 0 & 0 & 0 & 0 & 0 & 0 & 0 & 0 & 2 \\
0 & 0 & 1 & 0 & 0 & 0 & 0 & 0 & 0 & 0 & 0 & 0 & 0 & 0 & 0 & 1 \\
1 & 0 & 0 & 2 & 0 & 0 & 0 & 0 & 0 & 0 & 2 & 0 & 0 & 0 & 0 & 5 \\
0 & 0 & 1 & 0 & 0 & 0 & 1 & 0 & 0 & 0 & 0 & 0 & 0 & 0 & 0 & 2 \\
0 & 0 & 2 & 0 & 0 & 0 & 1 & 0 & 0 & 0 & 0 & 0 & 0 & 0 & 0 & 3 \\
0 & 0 & 0 & 0 & 0 & 0 & 0 & 1 & 0 & 0 & 0 & 0 & 0 & 0 & 0 & 1 \\
\end{tabular}

Moraceae

Ficus L. sp1

Moraceae

Ficus L. sp2

Moraceae

Ficus L. spp. 


\section{Family}

Species

Moraceae Ficus septic Burm.f.

Moringaceae

Moringa oleifera Lam.

Musaceae

Musa L. spp.

Moraceae

Eucalyptus deglupta Blume

Myrtaceae

Myrtaceae

Piperaceae

Poaceae

sidium guajava $\mathrm{L}$.

Syzygium malaccense (L.) Merr. \& L.M.Perry

Piper sp.

Bambusa Schreb. spp.

Podocapaceae Podocarpus neriifolius D.Don

Rubiaceae Timonius DC.spp.

ڤิ Rubicaceae Anthocephalus cadamba Miq.

Rubicaceae Coffea L. spp.

Rubicaceae Unk_3

\section{Rutaceae}

Rutaceae

Sapindaceae

Sapindaceae

Sterculiaceae

Tiliaceae

Ulmaceae

Ulmaceae

Ulmaceae

Citrus aurantiifolia (Christm.) Swingle

Citrus spp.

Ganophyllum falcatum Blume

Nephelium lappaceum L.

Kleinhovia hospita L.

Grewia L. sp.

Trema orientalis (L.) Blume

Trema tomentosa (Roxb.) H.Hara

Unk_1

unkonwn

Peukilisperma suafiolens

\begin{tabular}{rrrrrrrrrrrrrrrr}
0 & 0 & 0 & 0 & 0 & 0 & 1 & 1 & 0 & 0 & 0 & 0 & 0 & 0 & 0 & 2 \\
1 & 0 & 0 & 0 & 0 & 0 & 0 & 0 & 0 & 0 & 0 & 0 & 0 & 0 & 0 & 1 \\
0 & 0 & 12 & 14 & 20 & 4 & 0 & 0 & 39 & 10 & 16 & 5 & 0 & 3 & 2 & 125 \\
0 & 0 & 0 & 0 & 0 & 0 & 20 & 0 & 0 & 0 & 2 & 0 & 0 & 0 & 0 & 22 \\
1 & 0 & 0 & 1 & 0 & 1 & 0 & 0 & 0 & 0 & 3 & 1 & 0 & 0 & 0 & 7 \\
0 & 1 & 0 & 0 & 0 & 0 & 0 & 0 & 0 & 0 & 0 & 0 & 0 & 0 & 0 & 1 \\
0 & 1 & 0 & 0 & 0 & 0 & 0 & 0 & 0 & 0 & 0 & 0 & 0 & 0 & 0 & 1 \\
0 & 2 & 0 & 2 & 0 & 0 & 0 & 0 & 2 & 0 & 0 & 0 & 0 & 0 & 0 & 6 \\
0 & 0 & 1 & 0 & 0 & 0 & 0 & 0 & 0 & 0 & 0 & 0 & 0 & 0 & 0 & 1 \\
0 & 0 & 1 & 0 & 0 & 0 & 0 & 0 & 0 & 1 & 0 & 0 & 0 & 0 & 0 & 2 \\
0 & 1 & 0 & 0 & 0 & 0 & 0 & 3 & 0 & 0 & 0 & 0 & 0 & 0 & 0 & 4 \\
0 & 0 & 0 & 0 & 0 & 0 & 0 & 2 & 0 & 0 & 0 & 0 & 60 & 0 & 0 & 62 \\
0 & 0 & 0 & 0 & 0 & 0 & 0 & 1 & 0 & 0 & 0 & 0 & 0 & 0 & 0 & 1 \\
0 & 1 & 0 & 0 & 0 & 0 & 0 & 1 & 0 & 0 & 0 & 0 & 0 & 0 & 0 & 2 \\
0 & 0 & 0 & 3 & 0 & 0 & 0 & 0 & 0 & 0 & 0 & 0 & 0 & 0 & 0 & 3 \\
0 & 0 & 1 & 0 & 0 & 0 & 0 & 0 & 0 & 0 & 0 & 0 & 0 & 0 & 0 & 1 \\
0 & 0 & 0 & 1 & 0 & 0 & 0 & 0 & 0 & 0 & 0 & 0 & 0 & 0 & 0 & 1 \\
0 & 0 & 0 & 0 & 0 & 0 & 1 & 2 & 0 & 0 & 1 & 0 & 0 & 0 & 0 & 4 \\
0 & 0 & 0 & 0 & 0 & 0 & 1 & 0 & 0 & 0 & 0 & 0 & 0 & 0 & 0 & 1 \\
0 & 0 & 1 & 0 & 1 & 0 & 8 & 22 & 0 & 0 & 0 & 0 & 0 & 1 & 0 & 33 \\
0 & 0 & 1 & 0 & 0 & 0 & 0 & 0 & 0 & 0 & 0 & 0 & 0 & 0 & 0 & 1 \\
0 & 0 & 0 & 0 & 0 & 0 & 1 & 0 & 0 & 0 & 0 & 0 & 0 & 0 & 0 & 1 \\
0 & 0 & 0 & 0 & 1 & 0 & 0 & 0 & 0 & 0 & 0 & 0 & 0 & 0 & 0 & 1 \\
\hline
\end{tabular}




\begin{tabular}{|c|c|c|c|c|c|c|c|c|c|c|c|c|c|c|c|c|c|}
\hline Family & Species & 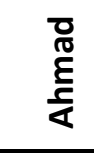 & 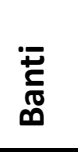 & 茂 & 总 & 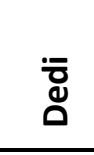 & 离 & 趸 & 矛 & $\underset{\mathrm{\Xi}}{\stackrel{\xi}{\sigma}}$ & 咅 & 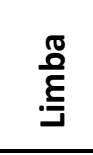 & 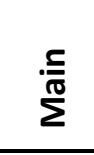 & 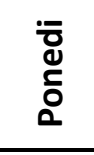 & 응 & $\overline{\check{c}}$ & $\begin{array}{l}\bar{\pi} \\
\stackrel{0}{0}\end{array}$ \\
\hline unkonwn & Policilus spermus & 0 & 0 & 0 & 0 & 0 & 0 & 0 & 1 & 0 & 0 & 0 & 0 & 0 & 0 & 0 & 1 \\
\hline unkonwn & Tersea & 0 & 0 & 0 & 0 & 0 & 0 & 0 & 0 & 0 & 0 & 0 & 5 & 0 & 0 & 0 & 5 \\
\hline unkonwn & Unk_3 & 0 & 0 & 1 & 0 & 0 & 0 & 0 & 0 & 0 & 0 & 0 & 0 & 0 & 0 & 0 & 1 \\
\hline unkonwn & Unk_4 & 0 & 0 & 0 & 0 & 0 & 0 & 0 & 0 & 1 & 0 & 0 & 0 & 0 & 0 & 0 & 1 \\
\hline unkonwn & Unk_5 & 0 & 0 & 0 & 4 & 0 & 0 & 0 & 0 & 0 & 0 & 0 & 0 & 0 & 0 & 0 & 4 \\
\hline Urticaceae & Pipturus argenteus (G.Forst.) Wedd. & 0 & 0 & 0 & 0 & 0 & 0 & 3 & 1 & 0 & 0 & 0 & 0 & 0 & 0 & 0 & 4 \\
\hline Urticaceae & Unk_2 & 0 & 0 & 0 & 0 & 0 & 0 & 0 & 4 & 0 & 0 & 0 & 0 & 0 & 0 & 0 & 4 \\
\hline Verbenaceae & Geunsia Blume spp. & 0 & 0 & 0 & 0 & 0 & 0 & 0 & 1 & 0 & 0 & 0 & 0 & 0 & 0 & 0 & 1 \\
\hline \multicolumn{2}{|c|}{ Number of all trees within one plantation } & 53 & 80 & 63 & 73 & 83 & 58 & 236 & 217 & 164 & 81 & 97 & 108 & 125 & 93 & 44 & 1575 \\
\hline & Proportion of Erythrina subumbrans. & 9,4 & 0 & 23,8 & 5,5 & 0 & 0 & 50,8 & 13,4 & 22 & 45,7 & 0 & 30,6 & 8 & 44,1 & 2,3 & 21 \\
\hline & Proportion of Gliricidia sepium & 54,7 & 75 & 6,3 & 31,5 & 68,7 & 91,4 & 25,4 & 27,6 & 22,6 & 7,4 & 60,8 & 55,6 & 44 & 41,9 & 70,5 & 40,2 \\
\hline & Proportion of both shade tree species & 64,2 & 75 & 30,2 & 37 & 68,7 & 91,4 & 76,3 & 41 & 44,5 & 53,1 & 60,8 & 86,1 & 52 & 86 & 72,7 & 61,2 \\
\hline
\end{tabular}


Table S3. Herbal plant species.

Names indicate the plantation identity. Column one and two show the taxonomic classification (Family, Species), columns 3 - 17 the site-specific species abundances and column 18 the sum of all sites ( 2 pages).

\begin{tabular}{|c|c|c|c|c|c|c|c|c|c|c|c|c|c|c|c|c|c|}
\hline Family & Species & 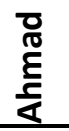 & 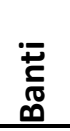 & $\frac{\bar{\pi}}{\overline{0}}$ & $\stackrel{\circ}{\frac{1}{0}}$ & $\begin{array}{l}\text { ர̄ } \\
\text { வ }\end{array}$ & $\begin{array}{l}\bar{\Sigma} \\
\text { อั } \\
\end{array}$ & 芆 & $\begin{array}{l}\overline{0} \\
\end{array}$ & 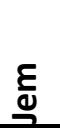 & 立 & 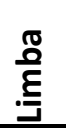 & $\frac{.5}{\sqrt{\pi}}$ & $\begin{array}{l}: \overline{0} \\
\stackrel{0}{0} \\
0 \\
0\end{array}$ & $\begin{array}{l}\text { 응 } \\
\text { 足 }\end{array}$ & 듬 & $\begin{array}{l}\bar{\pi} \\
\stackrel{0}{0}\end{array}$ \\
\hline Acanthaceae & Pseuderanthemum Radlk. spp. & 0 & 0 & 1 & 0 & 0 & 0 & 0 & 0 & 0 & 0 & 0 & 0 & 0 & 0 & 0 & 1 \\
\hline Armanthaceae & Armanthaceae spp. & 0 & 1 & 1 & 0 & 0 & 0 & 0 & 0 & 0 & 0 & 0 & 0 & 0 & 0 & 0 & 2 \\
\hline Aspleniaceae & Asplenium nidus & 0 & 0 & 0 & 1 & 0 & 0 & 0 & 0 & 0 & 0 & 0 & 0 & 0 & 0 & 0 & 1 \\
\hline Asteraceae & Ageratum conyzoides $\mathrm{L}$. & 1 & 1 & 1 & 1 & 1 & 1 & 1 & 1 & 1 & 1 & 1 & 1 & 1 & 1 & 1 & 15 \\
\hline Asteraceae & Bidens pilosa $\mathrm{L}$. & 1 & 1 & 1 & 1 & 1 & 1 & 0 & 1 & 0 & 1 & 1 & 1 & 1 & 1 & 0 & 12 \\
\hline Asteraceae & Crassocephalum crepidioides (Benth.) S.Moore & 1 & 1 & 1 & 0 & 1 & 0 & 1 & 0 & 0 & 1 & 0 & 0 & 1 & 1 & 1 & 9 \\
\hline Asteraceae & Elephantopus mollis Kunt. & 0 & 1 & 0 & 0 & 0 & 0 & 0 & 1 & 0 & 1 & 1 & 0 & 0 & 0 & 0 & 4 \\
\hline Asteraceae & Synedrella nodiflora Gaertn. & 1 & 0 & 0 & 1 & 0 & 1 & 0 & 0 & 1 & 0 & 1 & 0 & 1 & 1 & 0 & 7 \\
\hline Caryophyllaceae & Drymaria cordata (L.) Roem. \& Schult. & 0 & 0 & 0 & 0 & 1 & 0 & 1 & 0 & 0 & 1 & 1 & 0 & 0 & 0 & 1 & 5 \\
\hline Commelinaceae & Commelina nudiflora L. & 0 & 0 & 0 & 0 & 0 & 0 & 0 & 0 & 0 & 0 & 0 & 0 & 0 & 0 & 1 & 1 \\
\hline Convolvulaceae & Ipomoea batatas (L.) Lam. & 0 & 0 & 0 & 0 & 0 & 0 & 0 & 0 & 1 & 0 & 0 & 0 & 0 & 0 & 0 & 1 \\
\hline Cyperaceae & Cyperus cyperoides (L.) Kuntze & 1 & 0 & 0 & 0 & 1 & 1 & 0 & 0 & 0 & 0 & 0 & 1 & 1 & 0 & 0 & 5 \\
\hline Cyperaceae & Cyperus kyllingia Endl. & 0 & 0 & 0 & 0 & 1 & 0 & 1 & 0 & 0 & 1 & 1 & 1 & 1 & 1 & 1 & 8 \\
\hline Euphorbiaceae & Euphorbia heterophylla L. & 0 & 0 & 0 & 0 & 0 & 1 & 1 & 0 & 0 & 0 & 0 & 0 & 0 & 0 & 0 & 2 \\
\hline Limiaceae & Hyptis capitata Jaq. & 1 & 0 & 0 & 0 & 0 & 1 & 0 & 1 & 0 & 0 & 1 & 0 & 0 & 1 & 1 & 6 \\
\hline Loranthaceae Juss. & Loranthaceae Juss. Spp & 1 & 0 & 0 & 1 & 0 & 0 & 1 & 1 & 0 & 1 & 0 & 1 & 0 & 0 & 0 & 6 \\
\hline Lytraceae & Cuphea balsamona Cham. \& Schltdl. & 0 & 0 & 1 & 1 & 0 & 1 & 1 & 1 & 0 & 1 & 0 & 1 & 1 & 0 & 1 & 9 \\
\hline Oleanderceae & Nephrolepis Schott spp. & 0 & 0 & 1 & 0 & 0 & 0 & 0 & 0 & 0 & 0 & 0 & 0 & 0 & 0 & 0 & 1 \\
\hline other & Unknown Species 1 & 0 & 1 & 0 & 1 & 0 & 0 & 0 & 0 & 1 & 0 & 0 & 0 & 0 & 0 & 0 & 3 \\
\hline other & Unknown Species 2 & 0 & 0 & 0 & 0 & 0 & 0 & 0 & 0 & 1 & 0 & 0 & 0 & 0 & 0 & 0 & 1 \\
\hline Poaceae & Eleusine indica (L.) Gaertn. & 1 & 0 & 1 & 0 & 1 & 1 & 1 & 1 & 0 & 1 & 0 & 1 & 1 & 0 & 1 & 10 \\
\hline Poaceae & Imperata cylindrica (L.) P.Beauv. & 0 & 1 & 0 & 0 & 0 & 0 & 1 & 1 & 0 & 0 & 0 & 1 & 0 & 0 & 0 & 4 \\
\hline
\end{tabular}




\begin{tabular}{|c|c|c|c|c|c|c|c|c|c|c|c|c|c|c|c|c|c|}
\hline Family & Species & 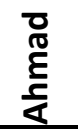 & $\begin{array}{l}\overline{\bar{c}} \\
\overline{\tilde{D}} \\
\end{array}$ & 元 & $\frac{8}{3}$ & 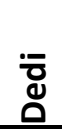 & פ̄ & בֶ & $\begin{array}{l}\overline{0} \\
\end{array}$ & $\underset{\mathrm{E}}{\mathrm{E}}$ & 咅 & 疍 & $\frac{\sqrt{\pi}}{\sum^{\pi}}$ & 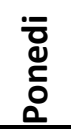 & 立 & 흠 & $\begin{array}{l}\overline{0} \\
\text { of }\end{array}$ \\
\hline Poaceae & Paspalum conjugatum P.J.Bergius & 0 & 1 & 0 & 0 & 1 & 1 & 0 & 1 & 1 & 0 & 1 & 1 & 0 & 1 & 1 & 9 \\
\hline Poaceae & Setaria palmifolia Stapf. & 0 & 0 & 1 & 0 & 0 & 0 & 0 & 0 & 1 & 0 & 0 & 0 & 0 & 1 & 0 & 3 \\
\hline Polygalaceae & Polygala sp. & 0 & 1 & 0 & 0 & 0 & 0 & 0 & 0 & 0 & 0 & 0 & 0 & 0 & 0 & 0 & 1 \\
\hline Rubiaceae & Borreria laevis Griseb. & 1 & 1 & 0 & 0 & 1 & 1 & 0 & 1 & 0 & 1 & 1 & 1 & 1 & 1 & 0 & 10 \\
\hline Thelypteridaceae & Christella parasitica (L.) H.Lev. & 0 & 0 & 0 & 0 & 0 & 0 & 1 & 0 & 1 & 0 & 0 & 0 & 0 & 0 & 0 & 2 \\
\hline Woodsiaceae & Diplazium esculentum (Retz.) Sw. & 1 & 0 & 1 & 0 & 1 & 0 & 0 & 0 & 0 & 0 & 1 & 0 & 1 & 1 & 0 & 6 \\
\hline Total & & 10 & 10 & 10 & 7 & 10 & 10 & 10 & 10 & 8 & 10 & 10 & 10 & 10 & 10 & 9 & 144 \\
\hline
\end{tabular}


Table S4. Discovered ant species.

Column one and two show the taxonomic classification (Family, Species), column three shows the abundances over all plantations derived from a quantitative ant baiting survey.

\begin{tabular}{|c|c|c|}
\hline Subfamily & Species & Abundance \\
\hline Dolichoderinae & Dolichoderus Sp. 3 & 210 \\
\hline Dolichoderinae & Tapinoma melanocephalum & 473 \\
\hline Dolichoderinae & Tapinoma Sp.1 & 1764 \\
\hline Dolichoderinae & Technomyrmex albipes & 3784 \\
\hline Formicinae & Anoplolepis gracilipes & 13868 \\
\hline Formicinae & Camponotus recticulatus & 260 \\
\hline Formicinae & Camponotus Sp. 1 & 2 \\
\hline Formicinae & Echinopla lineata & 1 \\
\hline Formicinae & Paratrechina Sp. 1 & 11 \\
\hline Formicinae & Paratrechina Sp. 4 & 248 \\
\hline Formicinae & Paratrechina Sp. 5 & 95 \\
\hline Formicinae & Paratrechina Sp. 6 & 33 \\
\hline Formicinae & Paratrechina Sp. 7 & 17 \\
\hline Formicinae & Paratrechina Sp. 8 & 782 \\
\hline Formicinae & Paratrechina Sp. 9 & 108 \\
\hline Formicinae & Polyrhachis abdominalis & 3 \\
\hline Formicinae & Polyrhachis dives & 22 \\
\hline Formicinae & Polyrhachis Sp. 4 & 19 \\
\hline Formicinae & Polyrhachis Sp. 10 & 3 \\
\hline Formicinae & Polyrhachis Sp. 13 & 9 \\
\hline Formicinae & Polyrhachis Sp. 5 & 11 \\
\hline Formicinae & Polyrhachis Sp. 8 & 15 \\
\hline Formicinae & Polyrhachis Sp. 9 & 1 \\
\hline Myrmicinae & Anillomyrma Sp. 2 & 5 \\
\hline Myrmicinae & Crematogaster Sp. 1 & 110 \\
\hline Myrmicinae & Crematogaster Sp. 3 & 1518 \\
\hline Myrmicinae & Monomorium floricola & 31 \\
\hline Myrmicinae & Monomorium Sp. 1 & 8335 \\
\hline Myrmicinae & Monomorium Sp. 2 & 191 \\
\hline Myrmicinae & Pheidole Sp. 10 & 6 \\
\hline Myrmicinae & Pheidole Sp. 11 & 23 \\
\hline Myrmicinae & Pheidole Sp. 2 & 4869 \\
\hline Myrmicinae & Pheidole Sp. 5 & 2344 \\
\hline Myrmicinae & Pheidole Sp. 9 & 246 \\
\hline Myrmicinae & Pheidologeton Sp. 1 & 52 \\
\hline Myrmicinae & Recurvidris Sp. 2 & 50 \\
\hline Myrmicinae & Solenopsis geminata & 2506 \\
\hline Myrmicinae & Tetramorium bicarinatum & 6 \\
\hline Myrmicinae & Tetramorium pasificum & 557 \\
\hline Myrmicinae & Tetramorium smithi & 9 \\
\hline Myrmicinae & Tetramorium Sp. 1 & 52 \\
\hline Myrmicinae & Tetramorium Sp. 12 & 171 \\
\hline
\end{tabular}




\begin{tabular}{llr}
\hline Subfamily & Species & Abundance \\
\hline Myrmicinae & Tetramorium Sp. 15 & 329 \\
Myrmicinae & Tetramorium Sp. 2 & 1465 \\
Myrmicinae & Tetramorium Sp. 5 & 3 \\
Myrmicinae & Tetramorium Sp. 6 & 12 \\
Myrmicinae & Tetramorium Sp. 8 & 1013 \\
Ponerinae & Odontomachus simillimus & 1 \\
Pseudomyrmecinae & Tetraponera Sp. 1 & 13 \\
\hline
\end{tabular}




\section{Table S5. Discovered bird species.}

Column one and two show the taxonomic classification (Family, Species), column three shows the abundances over all plantations derived from mist netting and point counts conducted by Bea Maas from September 2010 to June $2011^{1}$.

\begin{tabular}{|c|c|c|}
\hline Family & Species & Abundance \\
\hline Acanthizidae & Gerygone sulphurea & 23 \\
\hline Accipitriidae & Spizaetus lanceolatus & 1 \\
\hline Alcedinidae & Halcyon chloris & 46 \\
\hline Alcedinidae & Halcyon sancta & 1 \\
\hline Apodidae & Collocalia esculenta & 38 \\
\hline Apodidae & Collocalia vanikorensis & 9 \\
\hline Ardeidae & Ardea purpurea & 1 \\
\hline Ardeidae & Ardeola speciosa & 1 \\
\hline Ardeidae & Eggreta intermedia & 3 \\
\hline Arthamidae & Arthamus leucorhynchus & 6 \\
\hline Campephagidae & Coracina leucopygia & 12 \\
\hline Campephagidae & Coracina morio & 1 \\
\hline Campephagidae & Coracina temminckii & 1 \\
\hline Campephagidae & Lalage leucopygialis & 5 \\
\hline Columbidae & Chalcophaps indica & 3 \\
\hline Columbidae & Macropygia amboinensis & 1 \\
\hline Columbidae & Ptilinopus melanospila & 1 \\
\hline Columbidae & Ptilinopus superbus & 1 \\
\hline Corvidae & Corvus enca & 5 \\
\hline Cuculidae & Cacomantis sepulcralis & 4 \\
\hline Cuculidae & Centropus bengalensis & 5 \\
\hline Cuculidae & Chrysococcyx russatus & 2 \\
\hline Cuculidae & Cuculatus sparverioides & 1 \\
\hline Cuculidae & Cuculus saturatus & 5 \\
\hline Cuculidae & Phaenicophaeus calyorhynchus & 4 \\
\hline Dicaeidae & Dicaeum aureolimbatum & 175 \\
\hline Dicaeidae & Dicaeum celebicum & 609 \\
\hline Dicaeidae & Dicaeum nerhkorni & 33 \\
\hline Estrilididae & Lonchura malacca & 98 \\
\hline Estrilididae & Lonchura molucca & 25 \\
\hline Estrilididae & Lonchura punctulata & 10 \\
\hline Falconiidae & Falco molucensis & 1 \\
\hline Hemiprocnidae & Hemiprocne longipennis & 4 \\
\hline Hirundinidae & Hirundapus caudacutus & 2 \\
\hline Hirundinidae & Hirundo rustica & 2 \\
\hline Hirundinidae & Hirundo tahitica & 24 \\
\hline Meropidae & Merops ornatus & 2 \\
\hline Monarchidae & Hypothymis azurea & 1 \\
\hline Motacillidae & Motacilla cinerea & 4 \\
\hline
\end{tabular}




\begin{tabular}{|c|c|c|}
\hline Family & Species & Abundance \\
\hline Motacillidae & Motacilla flava & 1 \\
\hline Muscicapidae & Cyornis rufigastra & 12 \\
\hline Muscicapidae & Eumyias panayensis & 2 \\
\hline Muscicapidae & Ficedula hyperythra & 1 \\
\hline Nectariniidae & Aethopyga siparaja & 11 \\
\hline Nectariniidae & Antreptes malacensis & 18 \\
\hline Nectariniidae & Antreptes siparaja & 2 \\
\hline Nectariniidae & Nectarinia aspasia & 31 \\
\hline Nectariniidae & Nectarinia jugularis & 103 \\
\hline Oriolidae & Oriolus chinensis & 9 \\
\hline Pachycephalidae & Pachycephala sulfuriventer & 1 \\
\hline Passeridae & Passer montanus & 22 \\
\hline Petroicidae & Culicicapa helianthea & 1 \\
\hline Picidae & Dendrocopos temminckii & 4 \\
\hline Picnonotidae & Pycnonotus aurigaster & 2 \\
\hline Psittacidae & Loriculus exilis & 10 \\
\hline Psittacidae & Loriculus stigmatus & 11 \\
\hline Psittacidae & Trichoglossus flavoviridis & 12 \\
\hline Psittacidae & Trichoglossus ornatus & 5 \\
\hline Ralidae & Amaurornis isabellinus & 2 \\
\hline Ralidae & Gallirallus torquatus & 1 \\
\hline Rhipiduridae & Rhipidura teysmannii & 2 \\
\hline Strigidae & Ninox punctulata & 3 \\
\hline Sturnidae & Aplonis minor & 176 \\
\hline Sturnidae & Scissirostrum dubium & 119 \\
\hline Sylviidae & Cisticola exilis & 1 \\
\hline Timaliidae & Trichastoma celebense & 3 \\
\hline Turnicidae & Turnix suscitator & 3 \\
\hline Zosteropidae & Zosterops atrifrons & 150 \\
\hline Zosteropidae & Zosterops chloris & 1431 \\
\hline
\end{tabular}

${ }^{1}$ Maas, B. et al. (2015). Avian species identity drives predation success in tropical cacao agroforestry. Journal of Applied Ecology, 52(3), 735-743. 
Table S6. Ant and bird community.

Plantation: names indicate the plantation identity, SI: Shannon Index, SR(xxx): rarefied species richness with subsample size for rarefying the community in parenthesis, Ev: species evenness, Ab: sampled abundance.

\begin{tabular}{|c|c|c|c|c|c|c|c|c|}
\hline \multirow[b]{2}{*}{ Plantation } & \multicolumn{4}{|c|}{ Ants } & \multicolumn{4}{|c|}{ Birds } \\
\hline & SI & $S R(737)$ & Ev & $A b$ & SI & $S R(122)$ & Ev & $A b$ \\
\hline Ahmad & 1.49 & 12.87 & 0.58 & 3655 & 1.60 & 13.47 & 0.61 & 328 \\
\hline Banti & 1.63 & 12.00 & 0.66 & 737 & 1.95 & 13.78 & 0.74 & 167 \\
\hline Bolai & 1.29 & 13.00 & 0.50 & 2652 & 2.26 & 19.27 & 0.76 & 313 \\
\hline Ciko & 1.63 & 11.86 & 0.66 & 2720 & 2.00 & 14.15 & 0.75 & 258 \\
\hline Dedi & 1.60 & 11.28 & 0.66 & 2589 & 1.76 & 15.84 & 0.64 & 126 \\
\hline Deni & 1.47 & 12.07 & 0.59 & 2328 & 2.31 & 22.00 & 0.75 & 122 \\
\hline Dewa & 1.77 & 12.91 & 0.69 & 801 & 1.95 & 15.73 & 0.71 & 275 \\
\hline Febi & 0.31 & 4.93 & 0.20 & 5816 & 1.98 & 17.28 & 0.70 & 295 \\
\hline Jem & 1.18 & 13.06 & 0.46 & 2931 & 1.87 & 12.97 & 0.73 & 237 \\
\hline Kiki & 1.42 & 10.27 & 0.61 & 3961 & 1.03 & 8.48 & 0.48 & 232 \\
\hline Limba & 0.77 & 9.57 & 0.34 & 1867 & 1.96 & 14.63 & 0.73 & 199 \\
\hline Main & 1.60 & 12.42 & 0.63 & 2832 & 1.83 & 19.51 & 0.62 & 188 \\
\hline Ponedi & 1.48 & 11.84 & 0.60 & 3329 & 1.61 & 14.53 & 0.60 & 172 \\
\hline Robi & 0.98 & 11.08 & 0.41 & 3416 & 2.05 & 17.79 & 0.71 & 177 \\
\hline Toni & 0.31 & 6.48 & 0.16 & 6046 & 1.66 & 13.98 & 0.63 & 230 \\
\hline
\end{tabular}




\section{Fig S7. Open bat exclusion.}

We build bamboo scaffolds using locally distributed thick-walled bamboo with flat nodes. The diameter was between 15 and $7 \mathrm{~cm}$. Each scaffold builds up a rectangle with two long and two short sides. The top and both short sides were covered with one whole net. Each of the long sides was covered with a separate net.

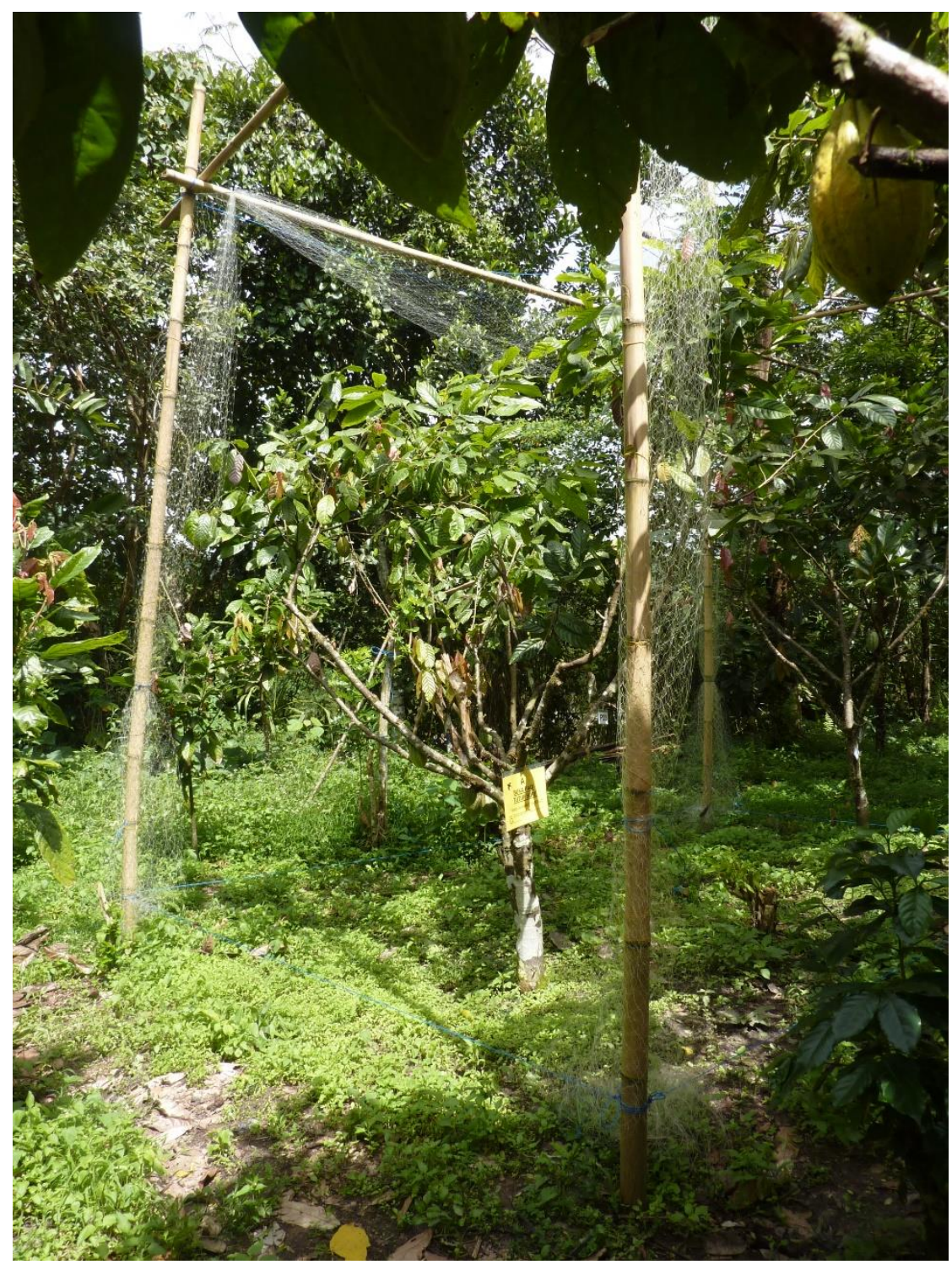




\section{Fig. S8. Conical-shaped ant exclusion rings (without insect glue).}

We measured the trunk diameter and cutted individual sized cone shaped exclusion rings for each tree. Contact area of trunk and plasik was sealed with foam material (light green). Plastic constructions were fixed with strips of the inner tube of motorcycle tyres. Positions of eclusion rings were shifted vertically for approximatly $5 \mathrm{~cm}$ every 6 month to avoid negativ effects on tree growth.

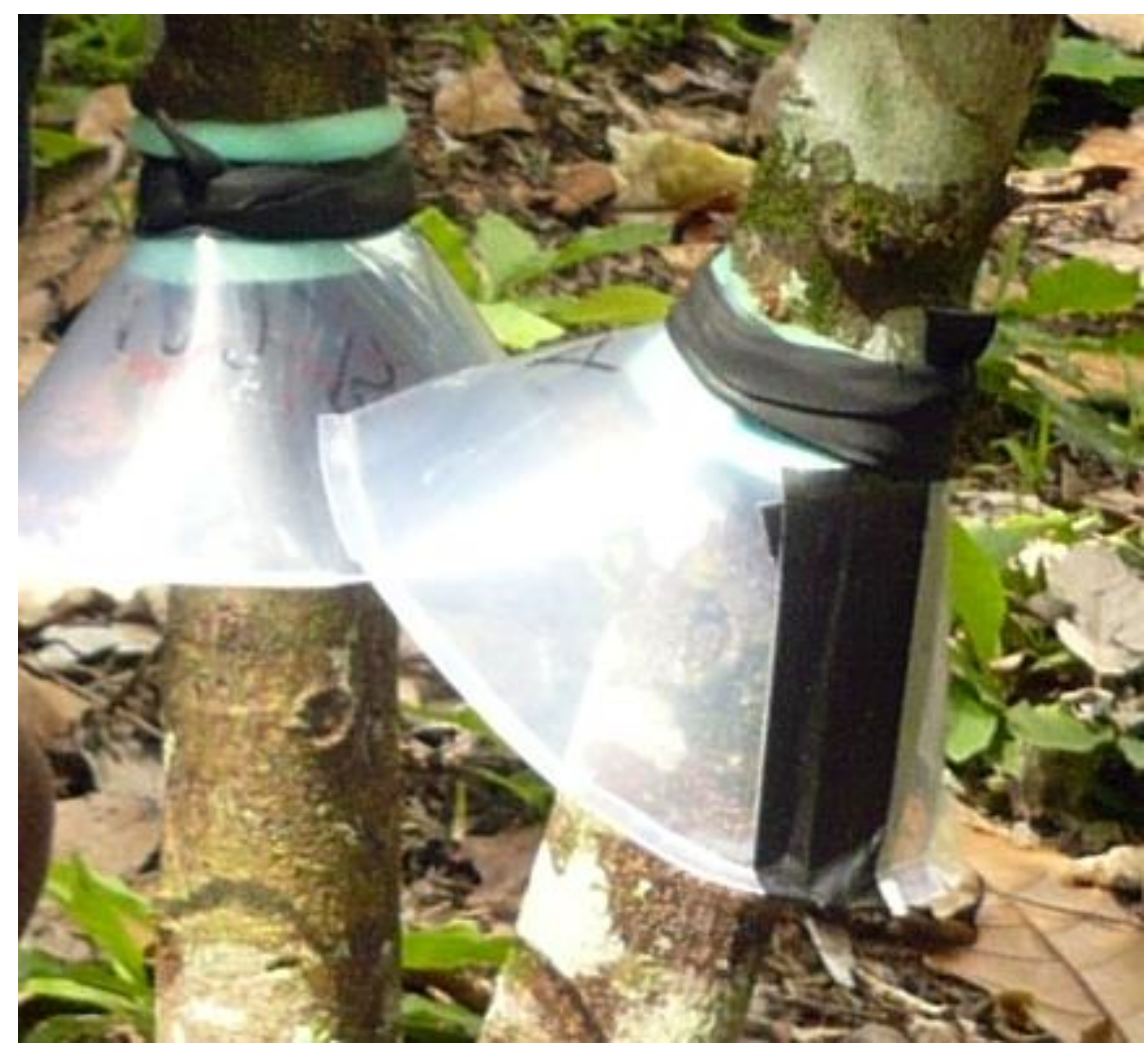


Table S9. Potentially yield influencing variables (additional to the predator exclusion).

Variables included in our full-model are indicated by a grey background colour. Despite not using them, other variables are reported to supply an overview of potential for further studies. Variable type: indicates the purpose of recording a particular variable, whereby design variables were manipulated through the experimental setting and covariates remained untouched but have been observed or measured. The column 'variable' names the each variable and category assigns a context the variable stands for. Recording level specifies the lowest level of measurement, sampling time the period when a measurement was done and recording interval and method specifies how long and details how a survey was conducted.

\begin{tabular}{|c|c|c|c|c|c|c|}
\hline $\begin{array}{l}\text { Variable } \\
\text { type }\end{array}$ & Variable & Category & Recording level & \begin{tabular}{|l|} 
Sampling \\
time
\end{tabular} & $\begin{array}{l}\text { Recording } \\
\text { Interval }\end{array}$ & Recording method \\
\hline Covariate & Temperature & Microclimate & Plantation & $\begin{array}{l}\text { May } 2011- \\
\text { June } 2012\end{array}$ & every 2 hours & $\begin{array}{l}\text { Automated electronic data logger, Thermochron }{ }^{\circledast} \text { iButton }{ }^{\circledast} \text { device } \\
\text { (DS1921G), } 2 \text { recorders per plantation }\end{array}$ \\
\hline Covariate & Leaf length & $\begin{array}{l}\text { Plantation } \\
\text { specific cacao } \\
\text { tree variety }\end{array}$ & Plantation & May 2012 & once & $\begin{array}{l}\text { We randomly selected } 80 \text { young and } 80 \text { old leaves ( } 40 \text { within the sun } \\
\text { and } 40 \text { in the shade) in each plantation ( } 1 \text { leaf }=1 \text { tree) and measured } \\
\text { the width at the widest part of the leaf with } 90^{\circ} \text { angle to the midrib. }\end{array}$ \\
\hline Covariate & Leaf weight & \begin{tabular}{|l|} 
Plantation \\
specific cacao \\
tree variety \\
\end{tabular} & Plantation & May 2012 & once & $\begin{array}{l}\text { We randomly selected } 80 \text { young and } 80 \text { old leaves ( } 40 \text { within the sun } \\
\text { and } 40 \text { in the shade) at each plantation ( } 1 \text { leaf }=1 \text { tree) and measured } \\
\text { the length along the midrib. }\end{array}$ \\
\hline Covariate & Leaf width & $\begin{array}{l}\text { Plantation } \\
\text { specific cacao } \\
\text { tree variety }\end{array}$ & Plantation & May 2012 & once & $\begin{array}{l}\text { We randomly selected } 80 \text { young and } 80 \text { old leaves ( } 40 \text { within the sun } \\
\text { and } 40 \text { in the shade) at each plantation ( } 1 \text { leaf }=1 \text { tree) and measured } \\
\text { its weight. (Micro scale } d=0.0001 \mathrm{~g} \text { ) }\end{array}$ \\
\hline Covariate & $\begin{array}{l}\text { pod } \\
\text { depth }\end{array}$ & $\begin{array}{l}\text { Plantation } \\
\text { specific cacao } \\
\text { tree variety }\end{array}$ & Plantation & June 2012 & once & $\begin{array}{l}\text { We selected } 80 \text { pods ( } 20 \text { small, } 20 \text { medium, } 20 \text { large, } 20 \text { ripe pods) } \\
\text { at each plantation and measured the biggest and the smallest of } \\
\text { each category for one tree. }\end{array}$ \\
\hline Covariate & pod length & $\begin{array}{l}\text { Plantation } \\
\text { specific cacao } \\
\text { tree variety }\end{array}$ & Plantation & June 2012 & once & $\begin{array}{l}\text { We selected } 80 \text { pods ( } 20 \text { small, } 20 \text { medium, } 20 \text { large, } 20 \text { ripe pods) } \\
\text { at each plantation and measured the biggest and the smallest of } \\
\text { each category for one tree. }\end{array}$ \\
\hline Covariate & pod surface area & $\begin{array}{l}\text { Plantation } \\
\text { specific cacao } \\
\text { tree variety }\end{array}$ & Plantation & June 2012 & once & calculated out of pod length and width \\
\hline
\end{tabular}




\begin{tabular}{|c|c|c|c|c|c|c|}
\hline $\begin{array}{l}\text { Variable } \\
\text { type }\end{array}$ & Variable & Category & Recording level & $\begin{array}{l}\text { Sampling } \\
\text { time }\end{array}$ & $\begin{array}{l}\text { Recording } \\
\text { Interval }\end{array}$ & Recording method \\
\hline Covariate & pod volume & \begin{tabular}{|l|} 
Plantation \\
specific cacao \\
tree variety
\end{tabular} & Plantation & June 2012 & once & calculated of pod length and width \\
\hline Covariate & pod width & \begin{tabular}{|l|} 
Plantation \\
specific cacao \\
tree variety
\end{tabular} & Plantation & June 2012 & once & $\begin{array}{l}\text { We selected } 80 \text { pods ( } 20 \text { small, } 20 \text { medium, } 20 \text { large, } 20 \text { ripe pods) } \\
\text { at each plantation and measured the biggest and the smallest of } \\
\text { each category for one tree. }\end{array}$ \\
\hline Covariate & $\begin{array}{l}\text { shadow tree } \\
\text { richness }\end{array}$ & $\begin{array}{l}\text { Plantation } \\
\text { specific habitat }\end{array}$ & Plantation & May 2012 & once & $\begin{array}{l}\text { All occurring individuals of all tree and shrub species at each } \\
\text { plantation were counted and identified by an Indonesian Botanist } \\
\text { (Hardianto Mangopo, IPB). Species richness was calculated in R. }\end{array}$ \\
\hline Covariate & shadow tree SI & $\begin{array}{l}\text { Plantation } \\
\text { specific habitat }\end{array}$ & Plantation & May 2012 & once & $\begin{array}{l}\text { All occurring individuals of all tree and shrub species at each } \\
\text { plantation were counted and identified by an Indonesian Botanist } \\
\text { (Hardianto Mangopo, IPB). Shannon index was calculated in R. }\end{array}$ \\
\hline Covariate & rain in $\mathrm{mm}$ & $\begin{array}{l}\text { Region within } \\
\text { the valley }\end{array}$ & $\begin{array}{l}\text { group of } \\
\text { plantations }\end{array}$ & $\begin{array}{l}\text { April } 2011 \\
\text { May } 2012 \\
\end{array}$ & $\begin{array}{l}\text { Daily in the } \\
\text { morning }\end{array}$ & $\begin{array}{l}\text { The daily precipitation was collected at } 5 \text { "regions" within the Napu } \\
\text { Valley. At each collection spot we used two } 20 \text { I water jerry can with } \\
\text { a funnel ( } 18.5 \mathrm{~cm} \text { diameter) fixed to the opening. }\end{array}$ \\
\hline Covariate & $\begin{array}{l}\text { distance to the } \\
\text { plantation border }\end{array}$ & Treatment cage & single tree & 2 times & $\begin{array}{l}\# 1) \quad \text { Jan-Feb } \\
2011 \quad \# 2) \text { Jan- } \\
\text { Feb } 2012\end{array}$ & $\begin{array}{l}\text { Distance between treatment cage centre and closest plantation } \\
\text { border measured with one flexible measuring tape. }\end{array}$ \\
\hline Covariate & $\begin{array}{l}\text { average branch } \\
\text { perimeter }\end{array}$ & Tree individual & single tree & 2 times & $\begin{array}{l}\# 1) \text { Jan-Feb } \\
2011 \quad \# 2 \text { ) Jan- } \\
\text { Feb } 2012\end{array}$ & $\begin{array}{l}\text { Perimeter measured with measuring tape at the branch base (direct } \\
\text { proximity to branching point) }\end{array}$ \\
\hline Covariate & crown volume & Tree individual & single tree & 2 times & $\begin{array}{l}\# 1) \quad J a n-F e b \\
2011 \quad \# 2 \text { ) Jan- } \\
\text { Feb } 2012\end{array}$ & $\begin{array}{l}\text { Measured with stiff measuring tape. } X=1 \text {. Measurement: Longest } \\
\text { possible horizontal crown diameter crossing the tree centre (trunk), } \\
Y=2 \text {. Measurement: Distance between tree centre and crown edge } \\
\text { with } 90^{\circ} \text { angle to the first measurement line. } Z=\text { tree height minus } \\
\text { height branching point. Used Equation: } V=(4 / 3)^{*} P I * X^{*} Y^{*} Z\end{array}$ \\
\hline
\end{tabular}




\begin{tabular}{|c|c|c|c|c|c|c|}
\hline $\begin{array}{l}\text { Variable } \\
\text { type }\end{array}$ & Variable & Category & Recording level & $\begin{array}{l}\begin{array}{l}\text { Sampling } \\
\text { time }\end{array} \\
\end{array}$ & $\begin{array}{l}\text { Recording } \\
\text { Interval }\end{array}$ & Recording method \\
\hline Covariate & DBH & Tree individual & single tree & 2 times & $\begin{array}{l}\text { \#1) Jan-Feb } \\
\text { 2011 \#2) Jan- } \\
\text { Feb 2009 }\end{array}$ & $\begin{array}{l}\text { Perimeter measured with measuring tape direct under the } \\
\text { branching point }\end{array}$ \\
\hline Covariate & height branching & Tree individual & single tree & 2 times & $\begin{array}{l}\# 1) \quad \text { Jan-Feb } \\
\text { 2011 \#2) Jan- } \\
\text { Feb 2011 }\end{array}$ & $\begin{array}{l}\text { Distance from the soil to the branching point (direct under) with } \\
\text { measuring tape }\end{array}$ \\
\hline Covariate & $\begin{array}{l}\text { number } \\
\text { branches }\end{array}$ & Tree individual & single tree & 2 times & $\begin{array}{l}\text { \#1) Jan-Feb } \\
2011 \text { \#2) Jan- } \\
\text { Feb 2012 } \\
\end{array}$ & Plain counted number of living branches \\
\hline Covariate & tree height & Tree individual & single tree & 2 times & $\begin{array}{l}\text { \#1) Jan-Feb } \\
\text { 2011 \#2) Jan- } \\
\text { Feb 2010 } \\
\end{array}$ & Measured with measuring tape till the tip of the highest branch \\
\hline $\begin{array}{l}\text { Design } \\
\text { variable }\end{array}$ & forest distance & Landscape type & Treatment* & $\begin{array}{l}\text { once, } \quad \text { gps } \\
\text { map update }\end{array}$ & $\begin{array}{l}\text { December } \\
2012\end{array}$ & $\begin{array}{l}\text { Recording of the GPS location of each experimental treatment, the } \\
\text { plantation border, and the rainforest edge. (GPS device: Garmin } \\
\text { Oregon 550t, Garmin International), }{ }^{*} \text { recorded for each } \\
\text { treatment individually, but the treatments are organized in } \\
\text { plantations. }\end{array}$ \\
\hline $\begin{array}{l}\text { Design } \\
\text { variable }\end{array}$ & High-level shade & $\begin{array}{l}\text { Plantation } \\
\text { management }\end{array}$ & Treatment & 2 times & $\begin{array}{l}\text { \#1) Mai } 2011 \\
\text { \#2) April } 2012 \\
\end{array}$ & $\begin{array}{l}\text { Digital hemispherical pictures direct above the cacao tree crown } \\
\text { (Canon G11 \& circular fish-eye lens), picture analyses conducted } \\
\text { with "CIMES-Fisheye"C } \quad 2009 \text { Jean-Michel Walter } \\
\text { Software (Package "GAP") http://jmnw.free.fr/. }\end{array}$ \\
\hline $\begin{array}{l}\text { Design } \\
\text { variable }\end{array}$ & Low-level shade & $\begin{array}{l}\text { Plantation } \\
\text { management }\end{array}$ & Treatment & 2 times & $\begin{array}{l}\# 1) \text { Mai } 2011 \\
\text { \#2) April } 2012 \\
\end{array}$ & $\begin{array}{l}\text { Digital hemispherical pictures under (at } 120 \mathrm{~cm} \text { ) the cacao tree } \\
\text { crown (Canon G11 \& circular fish-eye lens), Picture analyses } \\
\text { conducted with "CIMES-Fisheye"( } \bigcirc 2009 \text { Jean-Michel Walter } \\
\text { Software (Package "GAP") http://jmnw.free.fr/. }\end{array}$ \\
\hline
\end{tabular}


Table S10. Characteristics of experimental trees and plantations at treatment level.

Number: running number, plantation: site identity, treatment codes the predator exclusion treatment: $\mathrm{a}=$ bats excluded, $\mathrm{b}=$ birds excluded, $\mathrm{c}=$ birds and bats access, $\mathrm{d}=$ birds and bats excluded, $1=$ ants access, 2 = ants excluded, the other columns show covariates as used here. Covariate details are explained in Table S9. Canopy cover and Forest distance were measured at treatment level, daily mean temperature, non-crop tree richness, precipitation and fruit groove depth at plantation level, mean DBH and average branch perimeter at tree level. All values show averages at treatment or plot level. (4 Pages)

\begin{tabular}{|c|c|c|c|c|c|c|c|c|c|c|c|}
\hline 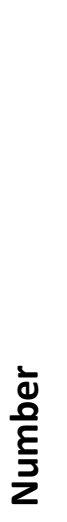 & $\begin{array}{l}\frac{5}{0} \\
\stackrel{0}{\pi} \\
\stackrel{0}{0} \\
\frac{\pi}{0}\end{array}$ & 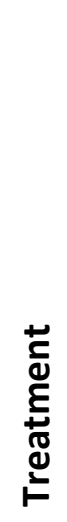 & 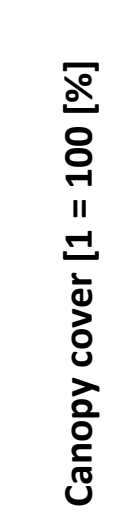 & 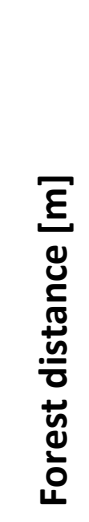 & 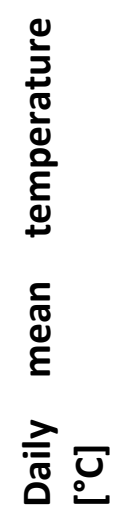 & 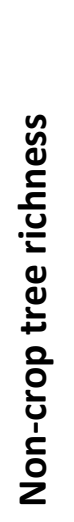 & 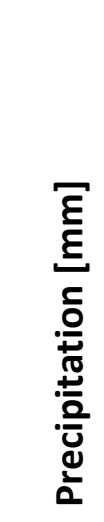 & 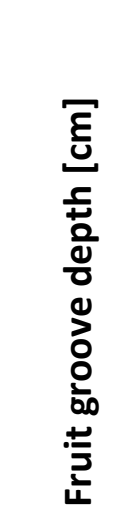 & 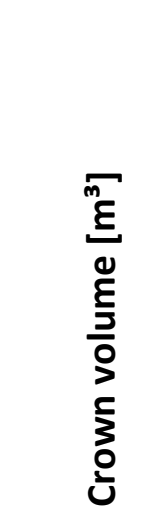 & 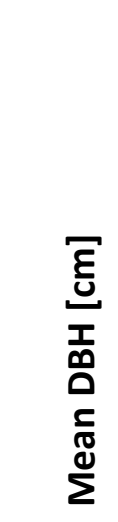 & 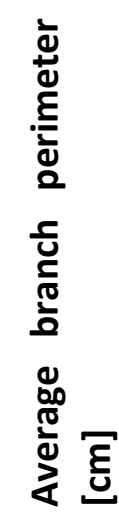 \\
\hline 1 & ahmad & a1 & 0.473 & 45 & 20.83 & 8 & 1281 & 0.395 & 8.026 & 22.15 & 13.81 \\
\hline 2 & ahmad & a2 & 0.603 & 26 & 20.83 & 8 & 1281 & 0.395 & 9.18 & 23.95 & 13.91 \\
\hline 3 & ahmad & b1 & 0.557 & 61 & 20.83 & 8 & 1281 & 0.395 & 9.958 & 23.25 & 15.69 \\
\hline 4 & ahmad & b2 & 0.298 & 63 & 20.83 & 8 & 1281 & 0.395 & 12.724 & 1.85 & 16.83 \\
\hline 5 & ahmad & c1 & 0.483 & 31 & 20.83 & 8 & 1281 & 0.395 & 12.301 & 26.49 & 16.38 \\
\hline 6 & ahmad & c2 & 0.302 & 74 & 20.83 & 8 & 1281 & 0.395 & 8.058 & 16.34 & 10.65 \\
\hline 7 & ahmad & $\mathrm{d} 1$ & 0.452 & 53 & 20.83 & 8 & 1281 & 0.395 & 6.4 & 18.83 & 12.38 \\
\hline 8 & ahmad & $d 2$ & 0.622 & 35 & 20.83 & 8 & 1281 & 0.395 & 8.827 & 19.36 & 13.45 \\
\hline 9 & & a1 & 0.405 & 205 & 20.48 & 14 & 1597 & 375 & 18.442 & 31.38 & 19.5 \\
\hline 10 & banti & a2 & 0.58 & 248 & 20.48 & 14 & 1597 & 0.375 & 12.639 & 22.59 & 16.86 \\
\hline 11 & banti & b1 & 0.637 & 239 & 20.48 & 14 & 1597 & 0.375 & 14.421 & 27.75 & 15.05 \\
\hline 12 & banti & b2 & 0.573 & 246 & 20.48 & 14 & 1597 & 0.375 & 16.637 & 32 & 22.58 \\
\hline 13 & banti & c1 & 0.663 & 254 & 20.48 & 14 & 1597 & 0.375 & 14.948 & 33.38 & 20.02 \\
\hline 14 & banti & c2 & 0.662 & 263 & 20.48 & 14 & 1597 & 0.375 & 19.257 & 26.73 & 18.18 \\
\hline 15 & banti & d1 & 0.67 & 255 & 20.48 & 14 & 1597 & 0.375 & 12.633 & 26.65 & 17.26 \\
\hline 16 & banti & $d 2$ & 0.562 & 266 & 20.48 & 14 & 1597 & 0.375 & 14.37 & 25.63 & 17.46 \\
\hline 17 & bolai & a1 & 0.232 & 224 & 20.4 & 24 & 1789 & 0.33 & 4.602 & 20.84 & 15.09 \\
\hline 18 & bolai & a2 & 0.35 & 241 & 20.4 & 24 & 1789 & 0.33 & 8.574 & 23.3 & 17.15 \\
\hline 19 & bolai & b1 & 0.355 & 232 & 20.4 & 24 & 1789 & 0.33 & 6.384 & 26.4 & 19.52 \\
\hline 20 & bolai & b2 & 0.237 & 208 & 20.4 & 24 & 1789 & 0.33 & 9.576 & 25.18 & 18.54 \\
\hline 21 & bolai & c1 & 0.44 & 223 & 20.4 & 24 & 1789 & 0.33 & 13.863 & 27.93 & 18.93 \\
\hline 22 & bolai & c2 & 0.39 & 213 & 20.4 & 24 & 1789 & 0.33 & 13.194 & 25.58 & 17.5 \\
\hline 23 & bolai & $\mathrm{d} 1$ & 0.378 & 225 & 20.4 & 24 & 1789 & 0.33 & 7.428 & 23.75 & 16.49 \\
\hline 24 & bolai & $d 2$ & 0.075 & 234 & 20.4 & 24 & 1789 & 0.33 & 9.339 & 26.83 & 19.48 \\
\hline
\end{tabular}




\begin{tabular}{|c|c|c|c|c|c|c|c|c|c|c|c|}
\hline 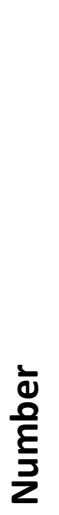 & 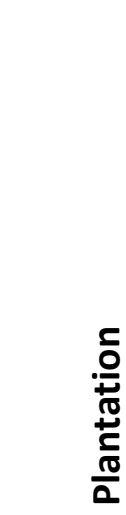 & 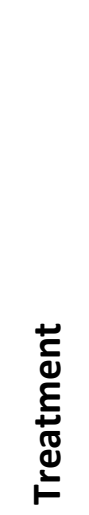 & 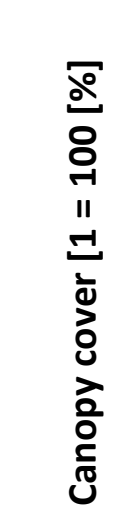 & 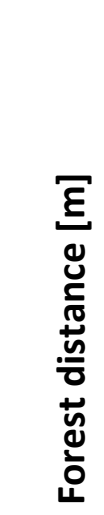 & 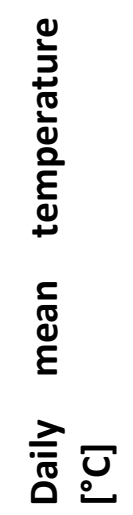 & 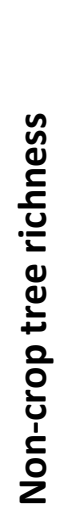 & 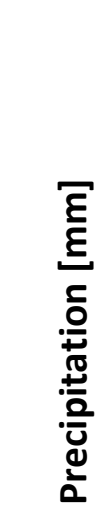 & 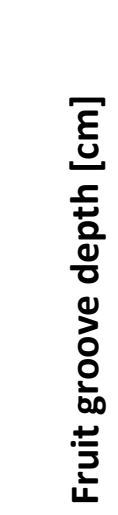 & 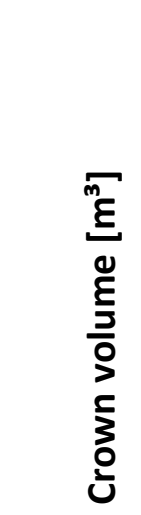 & 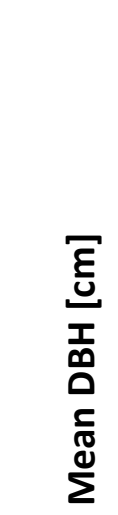 & 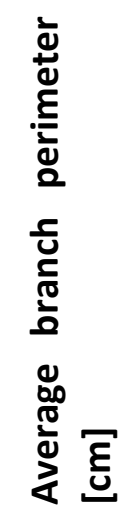 \\
\hline 25 & ciko & a1 & 0.312 & 861 & 21.09 & 16 & 1210 & 0.42 & 22.873 & 32.9 & 20.36 \\
\hline 26 & ciko & $a 2$ & 0.198 & 869 & 21.09 & 16 & 1210 & 0.42 & 14.838 & 31.13 & 19 \\
\hline 27 & ciko & b1 & 0.182 & 878 & 21.09 & 16 & 1210 & 0.42 & 18.269 & 39.4 & 27.83 \\
\hline 28 & ciko & b2 & 0.108 & 860 & 21.09 & 16 & 1210 & 0.42 & 13.408 & 37.88 & 21.75 \\
\hline 29 & ciko & c1 & 0.033 & 837 & 21.09 & 16 & 1210 & 0.42 & 12.454 & 32.7 & 20.56 \\
\hline 30 & ciko & c2 & 0.165 & 882 & 21.09 & 16 & 1210 & 0.42 & 19.778 & 26.73 & 16.94 \\
\hline 31 & ciko & d1 & 0.145 & 860 & 21.09 & 16 & 1210 & 0.42 & .546 & 35.3 & 22.28 \\
\hline 32 & ciko & $\mathrm{d} 2$ & 0.412 & 878 & 21.09 & 16 & 1210 & 0.42 & 13.222 & 29.59 & 21.19 \\
\hline 33 & dedi & a1 & 0.028 & 2705 & 21.07 & 7 & 1102 & 0.33 & 10.87 & 30.36 & 25.17 \\
\hline 34 & dedi & a2 & 0.142 & 2733 & 21.07 & 7 & 1102 & 0.33 & 12.816 & 37.03 & 27.8 \\
\hline 35 & dedi & b1 & 0.103 & 2714 & 21.07 & 7 & 1102 & 0.33 & 10.718 & 38.58 & 27.58 \\
\hline 36 & dedi & b2 & 0.14 & 2737 & 21.07 & 7 & 1102 & 0.33 & 18.567 & 40.7 & 28.55 \\
\hline 37 & dedi & c1 & 0.083 & 2747 & 21.07 & 7 & 1102 & 0.33 & 21.854 & 46.6 & 29.74 \\
\hline 38 & dedi & c2 & 0.015 & 2718 & 21.07 & 7 & 1102 & 0.33 & 497 & 6.93 & \\
\hline 39 & dedi & d1 & 0.133 & 2765 & 21.07 & 7 & 1102 & 0.33 & 9.792 & 35.58 & 25.06 \\
\hline 40 & dedi & $d 2$ & 0.017 & 2717 & 21.07 & 7 & 1102 & 0.33 & 9.906 & 34.88 & 26.33 \\
\hline 41 & deni & a1 & 0.365 & 2171 & 20.71 & 3 & 1072 & 0.325 & 8.686 & 17.83 & 11.61 \\
\hline 42 & deni & a2 & 0.442 & 2136 & 20.71 & 3 & 1072 & 0.325 & 10.201 & 23.03 & 15.38 \\
\hline 43 & deni & b1 & 0.525 & 2158 & 20.71 & 3 & 1072 & 0.325 & 12.261 & 20 & 12.61 \\
\hline 44 & deni & b2 & 0.448 & 2172 & 20.71 & 3 & 1072 & 0.325 & 9.922 & 17.29 & 11.97 \\
\hline 45 & deni & $\mathrm{c} 1$ & 0.365 & 2150 & 20.71 & 3 & 1072 & 0.325 & 14.988 & 26.13 & 17.1 \\
\hline 46 & deni & c2 & 0.473 & 2178 & 20.71 & 3 & 1072 & 0.325 & 17.209 & 24.98 & 16.61 \\
\hline 47 & deni & d1 & 0.542 & 2161 & 20.71 & 3 & 1072 & 0.325 & 14.619 & 26.7 & 14.92 \\
\hline 48 & deni & $d 2$ & 0.39 & 2147 & 20.71 & 3 & 1072 & 0.325 & 12.243 & 24.58 & 18.19 \\
\hline 49 & dewa & a1 & 0.335 & 102 & 21.25 & 18 & 1597 & 0.315 & 9.276 & 25.15 & 18.52 \\
\hline 50 & dewa & $a 2$ & 0.403 & 55 & 21.25 & 18 & 1597 & 0.315 & 16.285 & 33.5 & 22.15 \\
\hline 51 & dewa & b1 & 0.367 & 39 & 21.25 & 18 & 1597 & 0.315 & 11.199 & 34.13 & 22.39 \\
\hline 52 & dewa & b2 & 0.43 & 71 & 21.25 & 18 & 1597 & 0.315 & 18.616 & 31.73 & 21.64 \\
\hline 53 & dewa & c1 & 0.405 & 86 & 21.25 & 18 & 1597 & 0.315 & 16.812 & 27.53 & 20.57 \\
\hline 54 & dewa & c2 & 0.332 & 33 & 21.25 & 18 & 1597 & 0.315 & 19.334 & 33.13 & 23.19 \\
\hline 55 & dewa & d1 & 0.295 & 129 & 21.25 & 18 & 1597 & 0.315 & 16.083 & 30.05 & 28.46 \\
\hline 56 & dewa & $d 2$ & 0.492 & 42 & 21.25 & 18 & 1597 & 0.315 & 10.814 & 30.38 & 17.64 \\
\hline
\end{tabular}




\begin{tabular}{|c|c|c|c|c|c|c|c|c|c|c|c|}
\hline 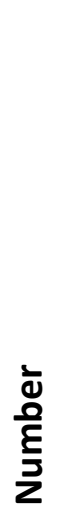 & $\begin{array}{l}\text { 음 } \\
\stackrel{2}{\pi} \\
\frac{1}{2} \\
\frac{\pi}{2}\end{array}$ & 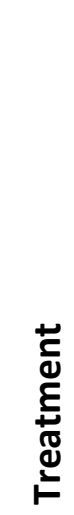 & 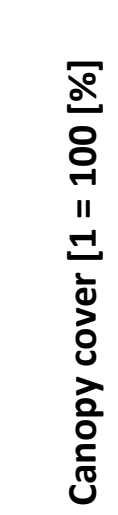 & 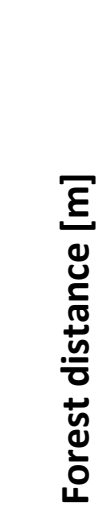 & 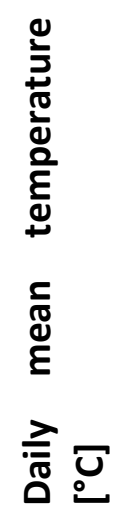 & 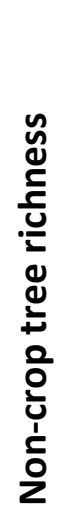 & 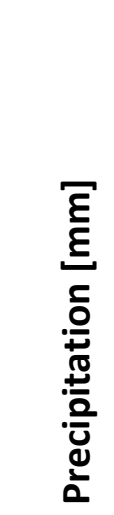 & 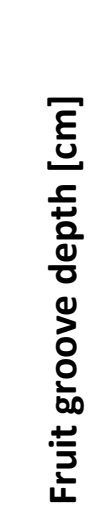 & 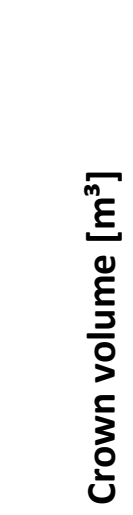 & 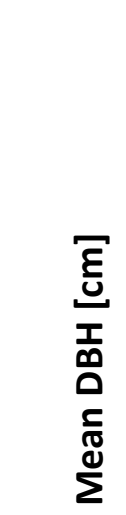 & 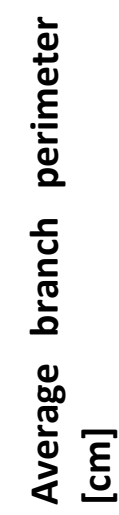 \\
\hline 57 & febi & a1 & 0.032 & 113 & 20.76 & 33 & 1597 & 0.44 & 14.41 & 28.88 & 18.81 \\
\hline 58 & febi & a2 & 0.113 & 75 & 20.76 & 33 & 1597 & 0.44 & 15.444 & 27.88 & 20.53 \\
\hline 59 & febi & b1 & 0.132 & 49 & 20.76 & 33 & 1597 & 0.44 & 19.978 & 32.08 & 23.79 \\
\hline 60 & febi & b2 & 0.148 & 124 & 20.76 & 33 & 1597 & 0.44 & 14.089 & 24.23 & 17.07 \\
\hline 61 & febi & c1 & 0.072 & 127 & 20.76 & 33 & 1597 & 0.44 & 16.386 & 23.73 & 15.33 \\
\hline 62 & febi & c2 & 0.07 & 141 & 20.76 & 33 & 1597 & 0.44 & 19.351 & 26.58 & 20.04 \\
\hline 63 & febi & d1 & 0.105 & 122 & 20.76 & 33 & 1597 & 0.44 & 887 & 29.93 & 21.06 \\
\hline 64 & febi & $d 2$ & 0.187 & 95 & 20.76 & 33 & 1597 & 0.44 & 15.356 & 27.45 & 20.95 \\
\hline 65 & jem & a1 & 0.262 & 317 & 20.45 & 14 & 1210 & 0.44 & 9.27 & 26.75 & 16.63 \\
\hline 66 & jem & a2 & 0.513 & 346 & 20.45 & 14 & 1210 & 0.44 & 9.636 & 23.4 & 14.89 \\
\hline 67 & jem & b1 & 0.183 & 309 & 20.45 & 14 & 1210 & 0.44 & 11.645 & 26.43 & 17.28 \\
\hline 68 & jem & b2 & 0.523 & 364 & 20.45 & 14 & 1210 & 0.44 & 7.492 & 21.6 & 14.96 \\
\hline 69 & jem & c1 & 0.552 & 321 & 20.45 & 14 & 1210 & 0.44 & 11.993 & 28.33 & 17.35 \\
\hline 70 & jem & c2 & 0.47 & 325 & 20.45 & 14 & 1210 & 0.44 & 23.188 & 33.35 & \\
\hline 71 & jem & d1 & 0.467 & 325 & 20.45 & 14 & 1210 & 0.44 & 11.469 & 27.38 & 16.56 \\
\hline 72 & jem & $d 2$ & 0.212 & 313 & 20.45 & 14 & 1210 & 0.44 & 13.433 & 28.63 & 17.13 \\
\hline 73 & kiki & a1 & 0.708 & 1102 & 20.7 & 10 & 1789 & 0.38 & 9.982 & 27.98 & 18.28 \\
\hline 74 & kiki & a2 & 0.437 & 1073 & 20.7 & 10 & 1789 & 0.38 & 15.092 & 31.23 & 21.17 \\
\hline 75 & kiki & b1 & 0.41 & 1062 & 20.7 & 10 & 1789 & 0.38 & 9.946 & 21.98 & 13.91 \\
\hline 76 & kiki & b2 & 0.425 & 1104 & 20.7 & 10 & 1789 & 0.38 & 15.192 & 25.55 & 20.11 \\
\hline 77 & kiki & c1 & 0.493 & 1055 & 20.7 & 10 & 1789 & 0.38 & 15.354 & 30.73 & 19.24 \\
\hline 78 & kiki & c2 & 0.3 & 1147 & 20.7 & 10 & 1789 & 0.38 & 26.168 & 30.23 & 21.46 \\
\hline 79 & kiki & d1 & 0.672 & 1122 & 20.7 & 10 & 1789 & 0.38 & 6.469 & 23.2 & 15.65 \\
\hline 80 & kiki & $\mathrm{d} 2$ & 0.528 & 1133 & 20.7 & 10 & 1789 & 0.38 & 15.187 & 30.78 & 20.52 \\
\hline 81 & limba & a1 & 0.367 & 563 & 20.49 & 13 & 1789 & 0.3 & 14.192 & 31.11 & 20.53 \\
\hline 82 & limba & $a 2$ & 0.37 & 582 & 20.49 & 13 & 1789 & 0.3 & 6.778 & 22.49 & 15.72 \\
\hline 83 & limba & b1 & 0.145 & 571 & 20.49 & 13 & 1789 & 0.3 & 7.783 & 25.43 & 17.6 \\
\hline 84 & limba & b2 & 0.598 & 567 & 20.49 & 13 & 1789 & 0.3 & 10.705 & 27.43 & 20.43 \\
\hline 85 & limba & c1 & 0.3 & 585 & 20.49 & 13 & 1789 & 0.3 & 15.077 & 34.41 & 23.01 \\
\hline 86 & limba & c2 & 0.143 & 584 & 20.49 & 13 & 1789 & 0.3 & 23.349 & 32.03 & 20.47 \\
\hline 87 & limba & d1 & 0.385 & 604 & 20.49 & 13 & 1789 & 0.3 & 9.71 & 26.8 & 17.32 \\
\hline 88 & limba & $d 2$ & 0.072 & 579 & 20.49 & 13 & 1789 & 0.3 & 11.287 & 30.3 & 19.85 \\
\hline
\end{tabular}




\begin{tabular}{|c|c|c|c|c|c|c|c|c|c|c|c|}
\hline 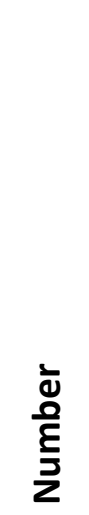 & $\begin{array}{l}\frac{5}{0} \\
\stackrel{0}{0} \\
\frac{0}{0} \\
\frac{\pi}{0} \\
\frac{\pi}{0}\end{array}$ & 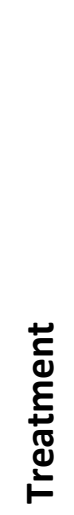 & 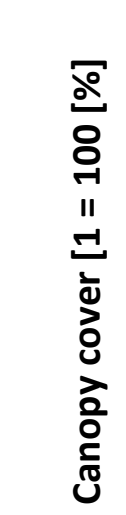 & 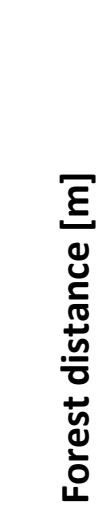 & 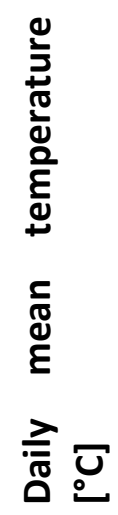 & 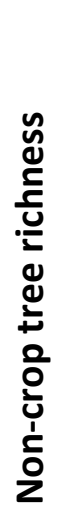 & 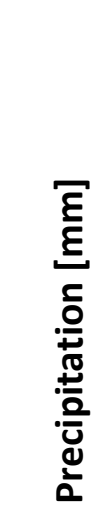 & 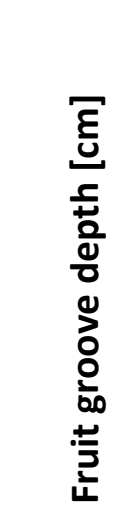 & 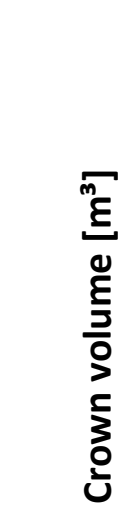 & 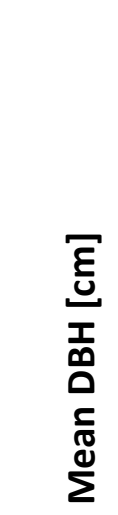 & 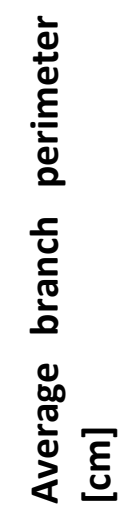 \\
\hline 89 & main & a1 & 0.252 & 2940 & 20.78 & 8 & 1072 & 0.435 & 11.345 & 26.28 & 19.72 \\
\hline 90 & main & a2 & 0.418 & 2984 & 20.78 & 8 & 1072 & 0.435 & 9.799 & 25.95 & 18.33 \\
\hline 91 & main & b1 & 0.463 & 2957 & 20.78 & 8 & 1072 & 0.435 & 5.704 & 15.74 & 11.41 \\
\hline 92 & main & b2 & 0.345 & 2977 & 20.78 & 8 & 1072 & 0.435 & 10.85 & 24.2 & 17.66 \\
\hline 93 & main & c1 & 0.387 & 2964 & 20.78 & 8 & 1072 & 0.435 & 21.484 & 27.98 & 18.82 \\
\hline 94 & main & $c 2$ & 0.057 & 2971 & 20.78 & 8 & 1072 & 0.435 & 20.399 & 33.2 & 22.45 \\
\hline 95 & main & $\mathrm{d} 1$ & 0.372 & 2975 & 20.78 & 8 & 1072 & 0.435 & 11.95 & 25.2 & 16.33 \\
\hline 96 & main & $\mathrm{d} 2$ & 0.308 & 2957 & 20.78 & 8 & 1072 & 0.435 & 10.13 & 24.05 & 17.93 \\
\hline 97 & ponedi & a1 & 0.428 & 2706 & 21.17 & 3 & 1072 & 0.255 & 4.973 & 18.35 & 12.83 \\
\hline 98 & & a2 & 0.273 & 2689 & 21.17 & 3 & 1072 & 255 & 6.02 & .13 & 14.08 \\
\hline 99 & ponedi & b1 & 0.283 & 2697 & 21.17 & 3 & 1072 & 0.255 & 7.025 & 21.2 & 15.6 \\
\hline 100 & ponedi & b2 & 0.185 & 2679 & 21.17 & 3 & 1072 & 255 & 963 & 1.35 & 14.84 \\
\hline 101 & ponedi & c1 & 0.453 & 2688 & 21.17 & 3 & 1072 & 0.255 & 5.913 & 23.53 & 15.63 \\
\hline 102 & ponedi & c2 & 0.328 & 2679 & 21.17 & 3 & 1072 & 0.255 & 10.317 & 22.85 & 16.6 \\
\hline 103 & ponedi & d1 & 0.383 & 2687 & 21.17 & 3 & 1072 & 0.255 & 9.205 & 24.93 & 15.4 \\
\hline 104 & ponedi & $\mathrm{d} 2$ & 0.147 & 2675 & 21.17 & 3 & 1072 & 0.255 & 10.74 & 22.58 & 15.94 \\
\hline 105 & robi & a1 & 0.193 & 1697 & 21.26 & 8 & 1102 & 0.315 & 6.56 & 20.68 & \\
\hline 106 & robi & $a 2$ & 0.34 & 1705 & 21.26 & 8 & 1102 & 0.315 & 13.412 & 27.83 & 20.04 \\
\hline 107 & robi & b1 & 0.243 & 1690 & 21.26 & 8 & 1102 & 0.315 & 10.862 & 26.95 & 19.09 \\
\hline 108 & robi & b2 & 0.097 & 1716 & 21.26 & 8 & 1102 & 0.315 & 13.198 & 21.88 & 15.45 \\
\hline 109 & robi & c1 & 0.142 & 1703 & 21.26 & 8 & 1102 & 0.315 & 13.606 & 23.26 & 16.26 \\
\hline 110 & robi & c2 & 0.175 & 1687 & 21.26 & 8 & 1102 & 0.315 & 22.339 & 35.13 & 24.18 \\
\hline 111 & robi & $\mathrm{d} 1$ & 0.18 & 1710 & 21.26 & 8 & 1102 & 0.315 & 9.383 & 22.78 & 16.47 \\
\hline 112 & robi & $d 2$ & 0.058 & 1703 & 21.26 & 8 & 1102 & 0.315 & 14.123 & 27.68 & 18.37 \\
\hline 113 & toni & a1 & 0.223 & 1723 & 20.48 & 5 & 1072 & 0.45 & 10.463 & 25.35 & 18.1 \\
\hline 114 & toni & a2 & 0.343 & 1748 & 20.48 & 5 & 1072 & 0.45 & 13.528 & 26.85 & 18.46 \\
\hline 115 & toni & b1 & 0.335 & 1748 & 20.48 & 5 & 1072 & 0.45 & 10.743 & 25.13 & 16.7 \\
\hline 116 & toni & b2 & 0.483 & 1717 & 20.48 & 5 & 1072 & 0.45 & 9.274 & 22.75 & 15.12 \\
\hline 117 & toni & c1 & 0.353 & 1731 & 20.48 & 5 & 1072 & 0.45 & 13.873 & 26.88 & 19.8 \\
\hline 118 & toni & c2 & 0.432 & 1736 & 20.48 & 5 & 1072 & 0.45 & 17.186 & 27.08 & 19.59 \\
\hline 119 & toni & $\mathrm{d} 1$ & 0.338 & 1747 & 20.48 & 5 & 1072 & 0.45 & 11.612 & 25.25 & 17.57 \\
\hline 120 & toni & $\mathrm{d} 2$ & 0.337 & 1726 & 20.48 & 5 & 1072 & 0.45 & 8.388 & 21.03 & 15.43 \\
\hline
\end{tabular}




\section{Table S11. Fruit development categories.}

Traits of cacao fruits assigned to four categories (small, medium, big and ripe) while conducting fruit surveys at 15 smallholder plantations in Central Sulawesi, Indonesia.

\begin{tabular}{|c|c|c|c|c|c|c|c|c|}
\hline \multirow[t]{2}{*}{ Fruit development classes } & \multicolumn{2}{|c|}{$\begin{array}{l}\text { Average } \\
\text { length }[\mathrm{cm}]\end{array}$} & \multicolumn{2}{|c|}{$\begin{array}{l}\text { Average } \\
\text { width }[\mathrm{cm}]\end{array}$} & \multicolumn{2}{|c|}{$\begin{array}{l}\text { Groove depth } \\
{[\mathrm{mm}]}\end{array}$} & \multirow[t]{2}{*}{ Colour } & \multirow[t]{2}{*}{$\begin{array}{l}\text { Surface } \\
\text { structure }\end{array}$} \\
\hline & mean & SD & mean & SD & mean & SD & & \\
\hline Small fruit & 5.3 & \pm 3.5 & 1.9 & \pm 1.2 & 0.1 & \pm 0.1 & green & Smooth \\
\hline Medium fruit & 13.1 & \pm 3.0 & 5.7 & \pm 1.2 & 0.3 & \pm 0.1 & green & rough, uneven \\
\hline Big fruit & 14.9 & \pm 3.3 & 7.5 & \pm 1.3 & 0.3 & \pm 0.1 & green, ripe spots & rough, uneven \\
\hline Ripe fruit & 15.4 & \pm 3.4 & 7.9 & \pm 1.2 & 0.4 & \pm 0.1 & ripe: red or yellow & smooth, even \\
\hline
\end{tabular}

\section{Table S12. Pest and disease categories.}

Diagnosis of pests and infections based on signs of fruits while conducting fruit surveys at 15 smallholder plantations in Central Sulawesi, Indonesia.

穴

\begin{tabular}{ll}
\hline Pest \& infection categories & Description \\
\hline Healthy & Fruits did not show any marks of pest or disease infection at the fruit surface. \\
Helopeltis sulawesi & Typical feeding damage necrosis are present at the fruit surface \\
Helopeltis sulawesi - strong & Typical feeding damage necrosis cover more than $50 \%$ the fruit surface. \\
Conopomorpha cramerella* & Typical, patchy ripening of the fruit. The pods show ripe and unripe spots, especially at the fruit tip. \\
& Fruits which are dead and have turned black. For small fruits often caused by "cherelle wilt". More mature \\
Dead / black fruit & fruits often die due to fungal infections.
\end{tabular}

Dead/black fruit Fungus fruit body (i.e. Phytophthora palmivora)

Dead fruits covered by fungus fruit bodies; white or greenish surface cover.

Fungus no fruit body (i.e. Phytophthora palmivora)

Dead fruits infected by fungus. Identified by brown mouldy-soft fruit parts.

Mammal (rat, squirrel, etc.)

Mechanical feeding damage

\footnotetext{
$*$ For harvested fruit CPB infestation was detected in opened fruits
} 


\section{S13 ANOVA Tables}

\section{Key to abbreviation:}

"Ant.excl" = Ant exclusion treatment

"Bird.excl" = Bird exclusion treatment

"Bat.excl" = Bat exclusion treatment

"dist_meter" = Distance to the forest margin

"ell_h" = Crown volume

"sh.hi" $\quad=\%$ shade-tree cover

"sh.hi.sq" = second order polynomial of $\%$ shade-tree cover

“:” = Interaction

".sc" = transformed to z-scores 


\section{Table S13.1 Total dry yield}

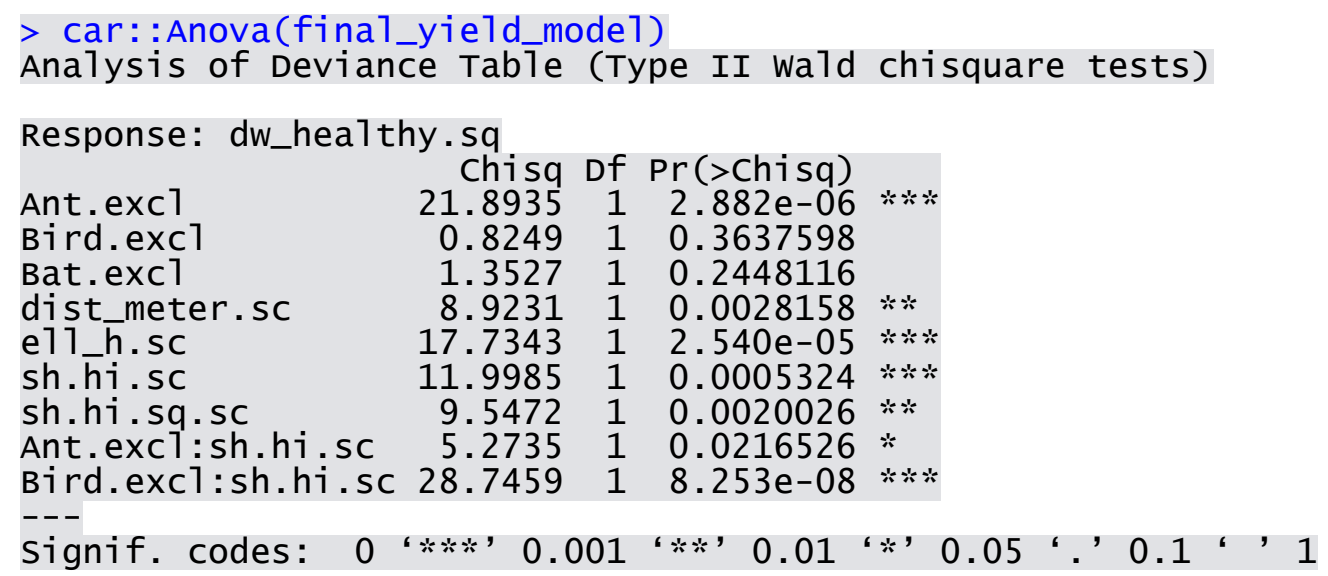

REML criterion at convergence: 1051.5

scaled residuals:

$\begin{array}{cccc}\text { Min } 1 \mathrm{Q} & \text { Median } & 3 \mathrm{Q} & \text { Max }\end{array}$

$\begin{array}{lllll}-2.03474 & -0.76691 & 0.07179 & 0.56739 & 2.30498\end{array}$

Random effects:

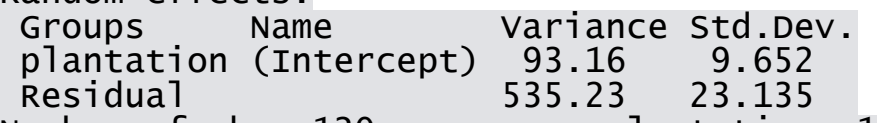

Number of obs: 120, groups: plantation, 15

Fixed effects:

\begin{tabular}{|c|c|c|c|c|c|}
\hline $\begin{array}{l}\text { (Intercept) } \\
\text { Ant.excl } \\
\text { Bird.excl } \\
\text { Bat.excl } \\
\text { dist_meter.sc } \\
\text { e11.h.sc } \\
\text { sh.hi.sc } \\
\text { sh.hi.sq.sc } \\
\text { Ant.excl:sh.hi.sc } \\
\text { Bird.exci:sh.hi.sc }\end{array}$ & $\begin{array}{r}\text { Estimate std. } \\
66.929 \\
-20.391 \\
4.028 \\
-5.265 \\
-10.101 \\
11.464 \\
31.429 \\
-28.294 \\
10.114 \\
-23.207\end{array}$ & $\begin{array}{l}\text { Error } \\
5.013 \\
4.325 \\
4.420 \\
4.527 \\
3.381 \\
2.722 \\
9.972 \\
9.157 \\
4.404 \\
4.328\end{array}$ & $\begin{array}{r}\mathrm{df} \\
53.320 \\
99.680 \\
100.670 \\
102.500 \\
13.130 \\
102.200 \\
92.090 \\
97.250 \\
102.810 \\
100.260\end{array}$ & $\begin{array}{r}t \text { value } \\
13.352 \\
-4.715 \\
0.911 \\
-1.163 \\
-2.987 \\
4.211 \\
3.152 \\
-3.090 \\
2.296 \\
-5.362\end{array}$ & $\begin{array}{r}\operatorname{Pr}(>|\mathrm{t}|) \\
<2 \mathrm{e}-16 \% \\
7.87 \mathrm{e}-06 \\
0.36437 \\
0.24751 \\
0.01040 * \\
5.48 \mathrm{e}-05 * \\
0.00219 * \\
0.00261 * \\
0.02368 * \\
5.30 \mathrm{e}-07 *\end{array}$ \\
\hline
\end{tabular}

Signif. codes: 0 “***; 0.001 “**, 0.01 ‘ $* 0.05$ ' 0.1 ' 0.1

Correlation of Fixed Effects:

Ant.exc1 $\quad-0.374$

Bird.exc1 $-0.461-0.058$

Bat.exc1 $-0.466-0.072$

e11_h.sc $\quad-0.201-0.200 \quad 0.289$

$\begin{array}{llll}\text { sh.h̆i.sc } & -0.029 & 0.021 & 0.028\end{array}$

$\begin{array}{llllll}\text { sh.hi.sq.sc } & 0.026 & -0.026 & -0.030 & 0.007 & 0.083\end{array}$

$\begin{array}{lllllllllll}\text { Brd.xcl:s.. } & -0.040 & 0.047 & -0.001 & 0.031 & -0.033 & -0.004 & -0.129 & -0.094 & -0.086\end{array}$ 


\section{Table S13.2 Fruit weight}

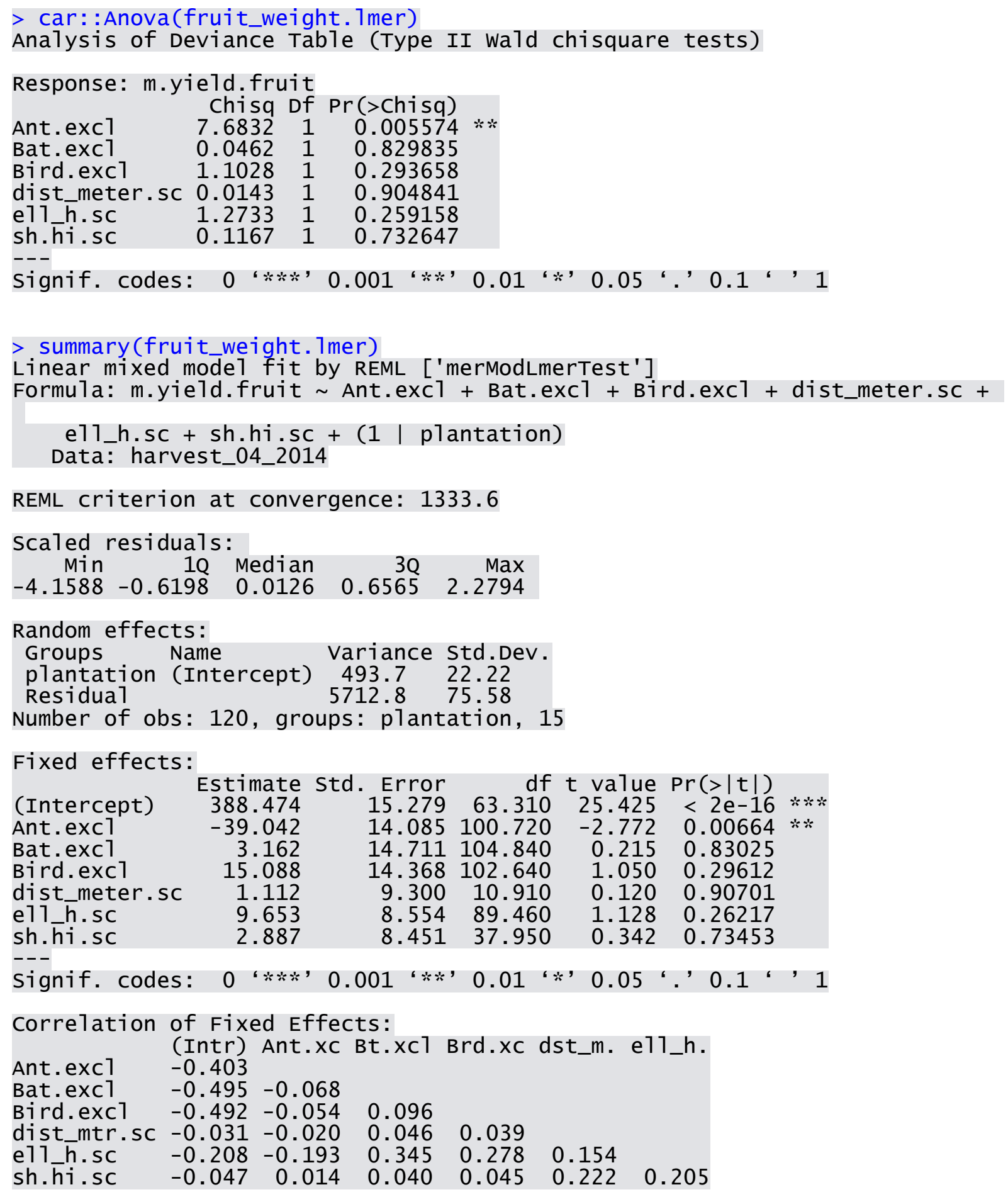




\section{Table S13.3 Number of fruits}

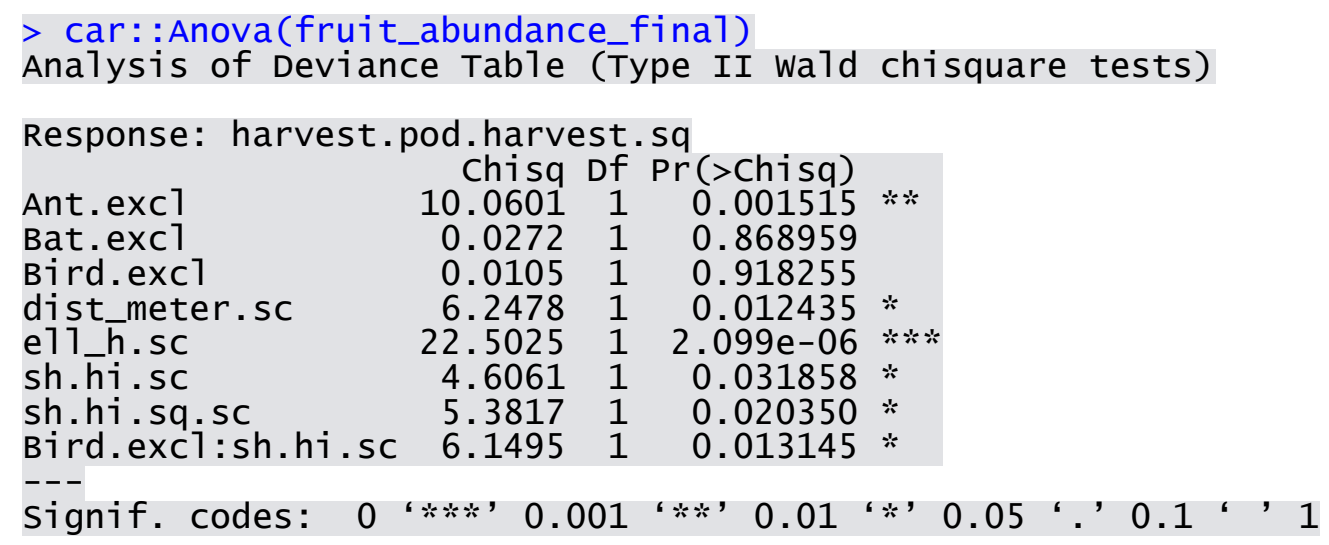




\section{Table S13.4 Flowers}

car: : Anova(flower1)
Analysis of Deviance Table (Type II wald chisquare tests)

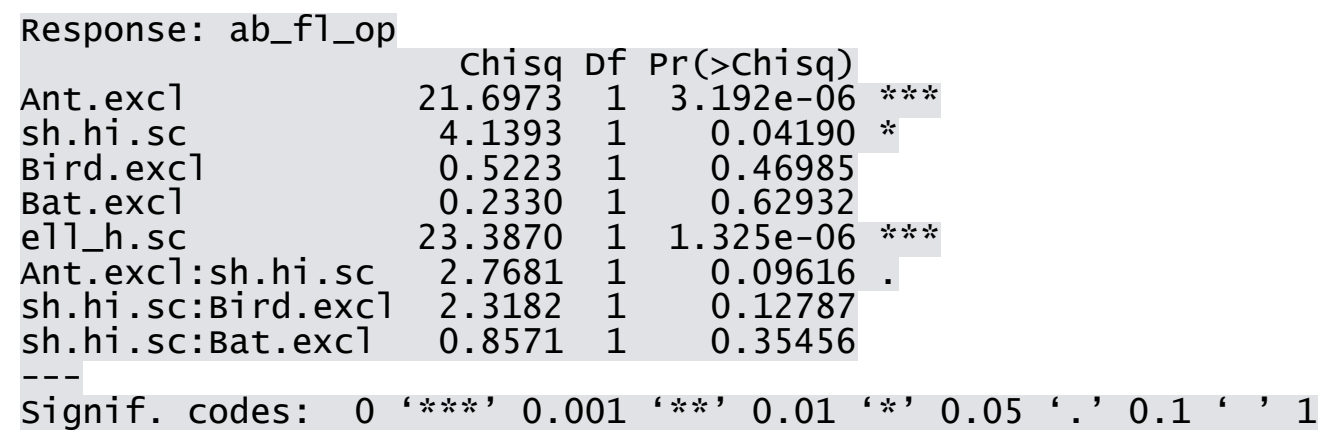

$>$ summary (flower 1$)$

Generalized linear mixed model fit by maximum likelihood (Laplace Approxima tion) ['glmerMod']

Family: poisson ( 1 og )

Formula: ab_f1_op Ant.excl * sh.hi.sc + Bird.excl * sh.hi.sc + Bat.excl * sh.hi.sc + el1_h.sc + (1 | plantation $)+(1 \mid$ obs $)$

Data: harvest_04_2014

Contro1: glmercontro1 (optimizer = "bobyqa")

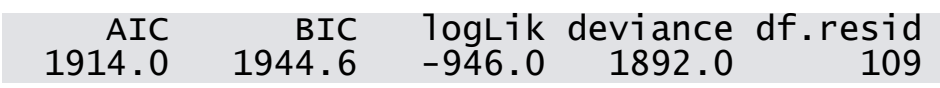

Scaled residuals:

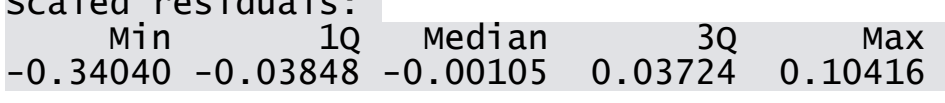

Random effects:

$\begin{array}{llll}\text { Groups } & \text { Name } & \text { Variance } & \text { Std.Dev. } \\ \text { obs } & \text { (Intercept) } & 0.1941 & 0.4406 \\ \text { plantation (Intercept) } & 0.1276 & 0.3572 \\ \text { Number of obs: 120, groups: obs, } & \text { 120; plantation, } 15\end{array}$

Fixed effects:

\begin{tabular}{|c|c|c|c|c|c|}
\hline $\begin{array}{l}\text { (Intercept) } \\
\text { Ant.exc1 } \\
\text { sh.hi.sc } \\
\text { Bird.exc1 } \\
\text { Bat.exc1 } \\
\text { e11_h.sc } \\
\text { Ant.excl:sh.hi.sc } \\
\text { sh.hi.sc:Bird.exc1 } \\
\text { sh.hi.sc:Bat.excl }\end{array}$ & $\begin{array}{r}\text { Estimate } \\
7.41600 \\
-0.38843 \\
-0.17291 \\
-0.06161 \\
-0.04301 \\
0.26951 \\
0.13844 \\
-0.12714 \\
0.07765\end{array}$ & $\begin{array}{r}\text { Std. Error } \\
0.12430 \\
0.08292 \\
0.09475 \\
0.08494 \\
0.08736 \\
0.05573 \\
0.08321 \\
0.08350 \\
0.08388\end{array}$ & $\begin{array}{r}\text { z value } \\
59.66 \\
-4.68 \\
-1.82 \\
-0.73 \\
-0.49 \\
4.84 \\
1.66 \\
-1.52 \\
0.93\end{array}$ & $\begin{array}{r}\operatorname{Pr}(>|z|) \\
<2 \mathrm{e}-16 \\
2.81 \mathrm{e}-06 \\
0.0680 \\
0.4683 \\
0.6225 \\
1.32 \mathrm{e}-06 \\
0.0962 \\
0.1279 \\
0.3546\end{array}$ & $\left\{\begin{array}{l}* * * \\
* * * \\
* * * \\
.\end{array}\right.$ \\
\hline
\end{tabular}

signif. codes: 0 “***, 0.001 ‘**, $0.01 ، *, 0.05 ،, 0.1 ،, 1$

Correlation of Fixed Effects:

Ant.excl (Intr) Ant.xc sh.h.s Brd.xc Bt.xcl e11_h. An.:.. sh.h.sc:Br.

sh.hi.sc $\quad-0.003 \quad 0.028$

Bird.exc1 $\quad-0.359-0.068-0.022$

$\begin{array}{llll}\text { Bat.exc1 } & -0.364-0.083-0.001\end{array}$

$\begin{array}{llll}\text { e11_h.sc } & -0.167 & -0.215 & 0.067\end{array}$

$\begin{array}{lrrr}\text { Ant.xCl:s.. } & 0.004 & -0.016 & -0.444 \\ \text { sh.h.sC:Br. } & -0.033 & 0.042 & -0.384\end{array}$

sh.h.sC:Bt. $-0.003-0.032-0.375$

0.116

$0.309 \quad 0.380$

$\begin{array}{lll}0.061 & -0.011 & 0.045\end{array}$

$\begin{array}{llll}0.002 & 0.042 & 0.025 & -0.064\end{array}$

$\begin{array}{lllll}0.025 & -0.010 & -0.017 & -0.001 & -0.139\end{array}$ 


\section{Table S13.5 Aborted small fruits}

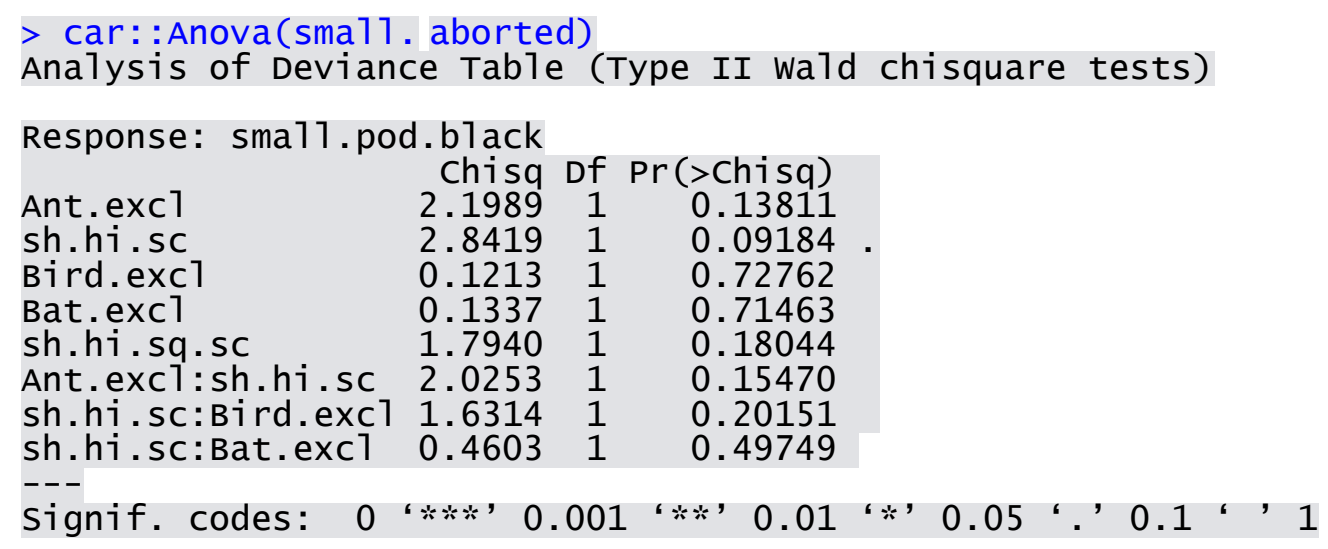




\section{Table S13.6 Not infested fruits}

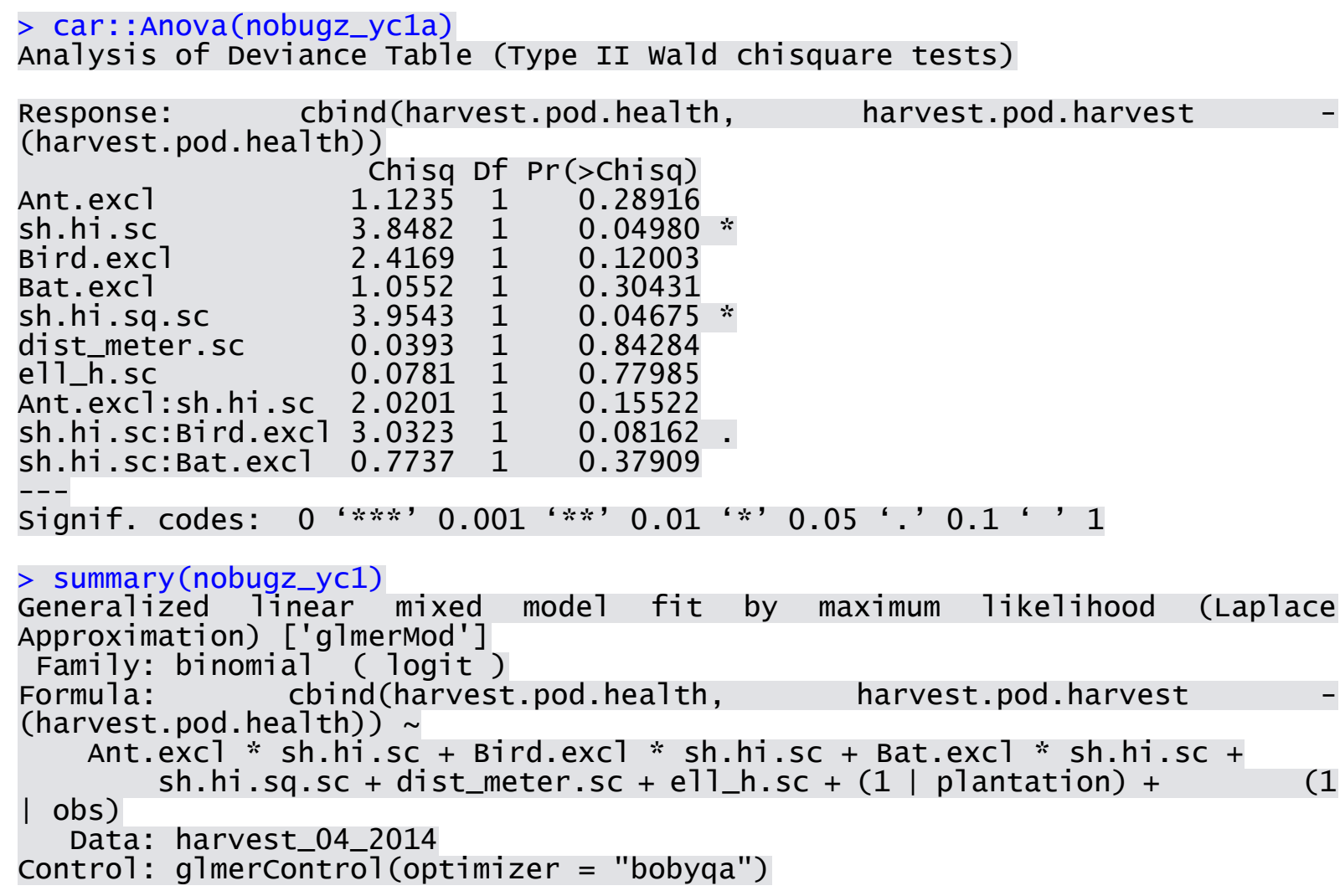

$\begin{array}{lrrrr} & \text { Estimate } & \text { Std. Error } & \text { value } & \operatorname{Pr}(>|z|) \\ \text { (Intercept) } & -2.10403 & 0.27236 & -7.725 & 1.12 \mathrm{e}-14 \\ \text { Ant.exc1 } & -0.16274 & 0.15265 & -1.066 & 0.2864 \\ \text { sh.hi.sc } & 0.73253 & 0.41011 & 1.786 & 0.0741 \\ \text { Bird.excl } & 0.23771 & 0.15909 & 1.494 & 0.1351 \\ \text { Bat.excl } & -0.17060 & 0.15951 & -1.070 & 0.2848 \\ \text { sh.hi.sq.sc } & -0.75849 & 0.38143 & -1.989 & 0.0468 * \\ \text { dist_meter.sc } & 0.04760 & 0.24008 & 0.198 & 0.8428 \\ \text { e11_h.sc } & -0.02846 & 0.10181 & -0.280 & 0.7799 \\ \text { Ant.excl:sh.hi.sc } & 0.24389 & 0.17159 & 1.421 & 0.1552 \\ \text { sh.hi.sc:Bird.exc1 } & -0.29387 & 0.16876 & -1.741 & 0.0816 . \\ \text { sh.hi.sc:Bat.excl } & -0.14981 & 0.17032 & -0.880 & 0.3791\end{array}$




\section{Table S13.7 Caterpillars 4-10mm}

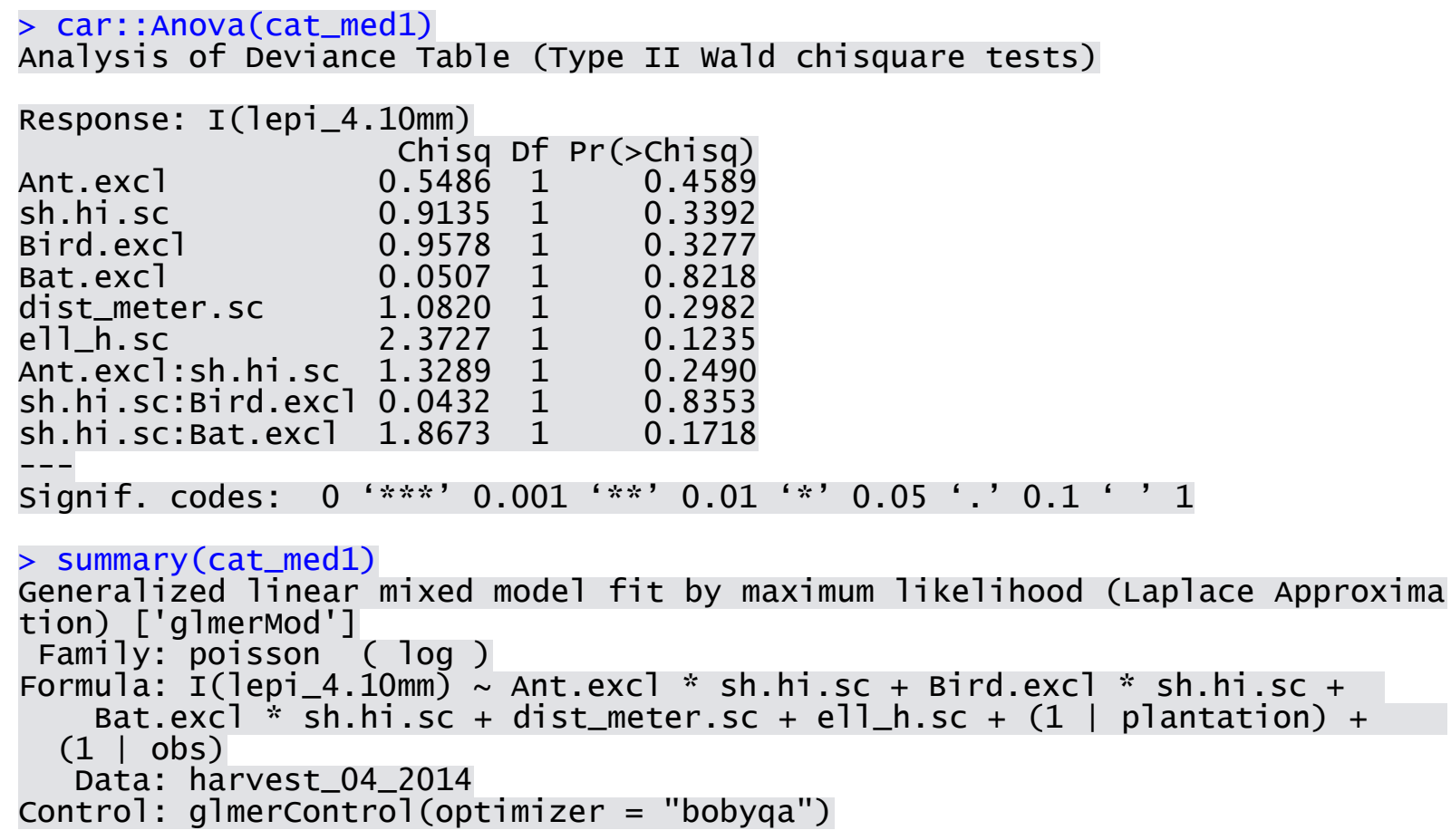

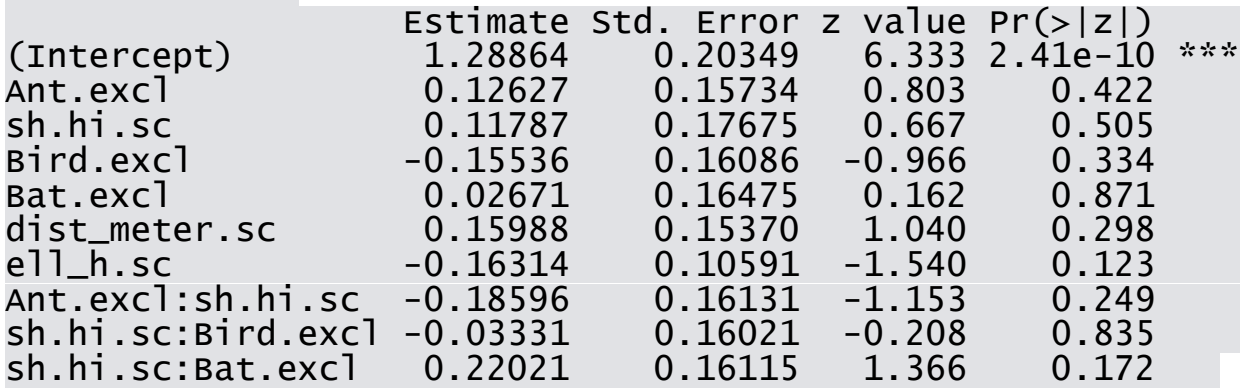




\section{Table S13.9 Coleoptera $>10 \mathrm{~mm}$}

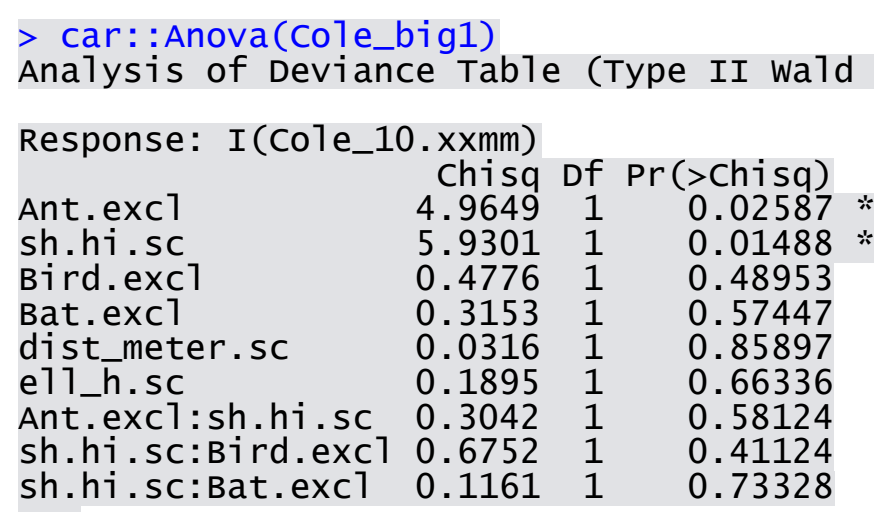

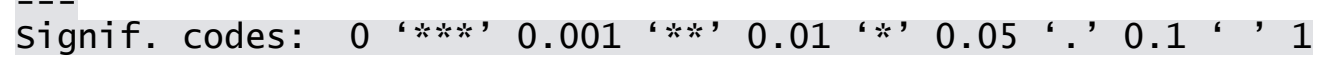

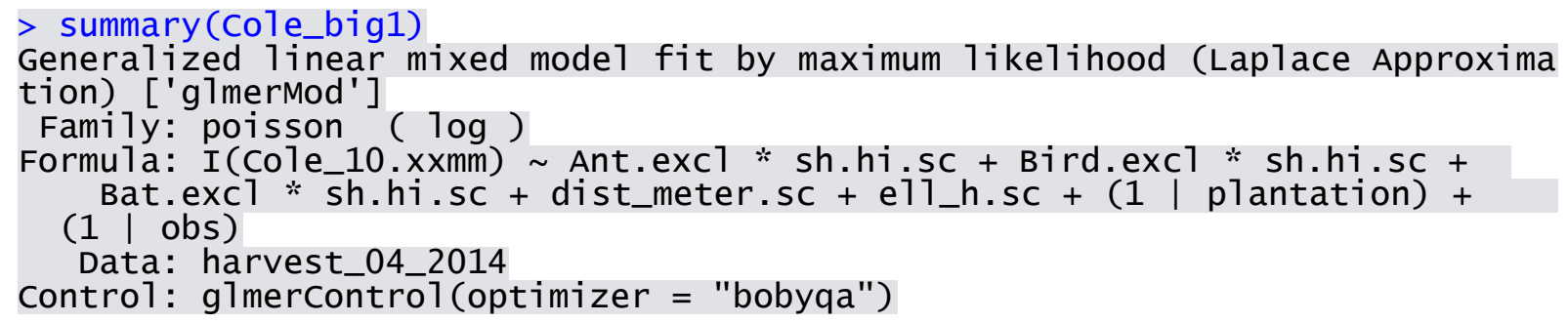

$\begin{array}{rrrrr}\text { AIC } & \text { BIC } & \text { logLik deviance } & \text { df.resid } \\ 472.1 & 505.5 & -224.0 & 448.1 & 108\end{array}$

Scaled residuals:

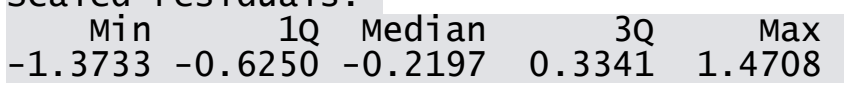

Random effects:

$\begin{array}{llll}\text { Groups } & \text { Name } & \text { Variance } & \text { Std.Dev. } \\ \text { obs } & \text { (Intercept) } & 0.3896 & 0.6242\end{array}$

plantation (Intercept) $1.0974 \quad 1.0475$

Number of obs: 120, groups: obs, 120; plantation, 15

Fixed effects:

$\begin{array}{lrrrr} & \text { Estimate } & \text { Std. Error } & \text { value } & \operatorname{Pr}(>|z|) \\ \text { (Intercept) } & -0.13883 & 0.35014 & -0.396 & 0.6917 \\ \text { Ant.exc1 } & 0.41021 & 0.19165 & 2.140 & 0.0323 \\ \text { sh.hi.sc } & -0.36036 & 0.23270 & -1.549 & 0.1215 \\ \text { Bird.excl } & 0.16127 & 0.19697 & 0.819 & 0.4129 \\ \text { Bat.exc1 } & 0.10499 & 0.20364 & 0.516 & 0.6061 \\ \text { dist_meter.sc } & 0.05170 & 0.29098 & 0.178 & 0.8590 \\ \text { e11_h.sc } & -0.05763 & 0.13241 & -0.435 & 0.6634 \\ \text { Ant.exc1:sh.hi.sc } & -0.11035 & 0.20006 & -0.552 & 0.5812 \\ \text { sh.hi.sc:Bird.exc1 } & 0.16662 & 0.20277 & 0.822 & 0.4112 \\ \text { sh.hi.sc:Bat.exc1 } & -0.06739 & 0.19775 & -0.341 & 0.7333\end{array}$




\section{Table S13.10 Auchenorrhyncha}

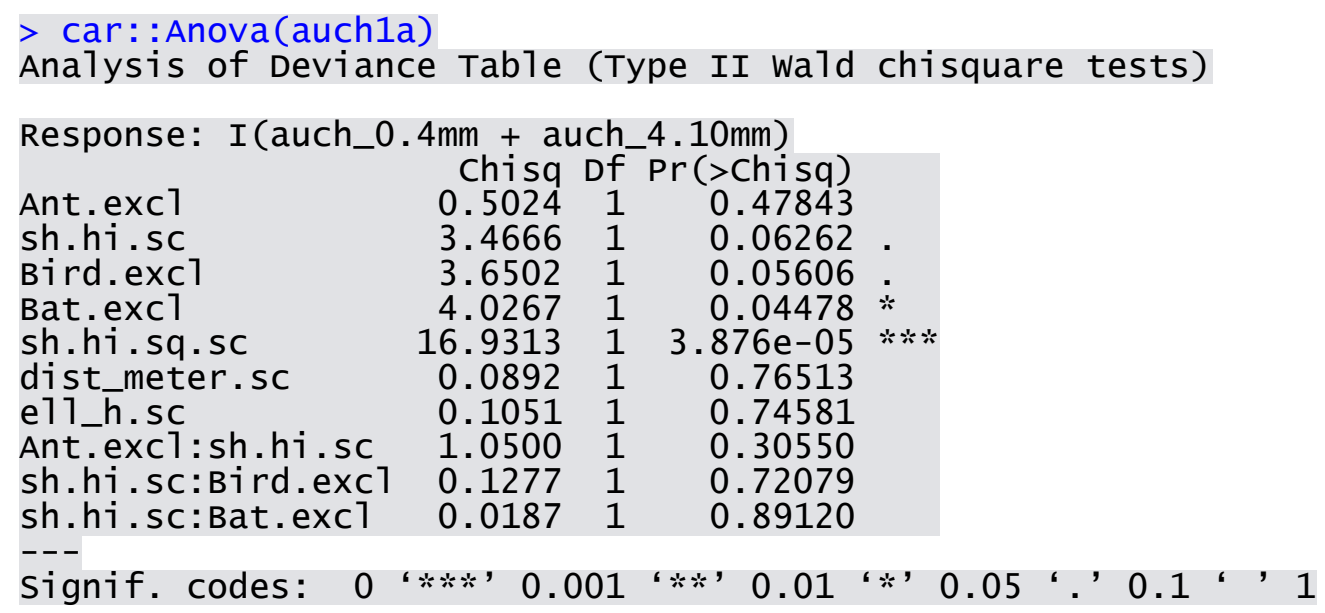

Fixed effects:

\begin{tabular}{|c|c|c|c|c|c|}
\hline $\begin{array}{l}\text { (Intercept) } \\
\text { Ant.exc1 } \\
\text { sh.hi.sc } \\
\text { Bird.exc1 } \\
\text { Bat.exc1 } \\
\text { sh.hi.sq.sc } \\
\text { dist_meter.sc } \\
\text { e11_h.sc } \\
\text { Ant.exc1:sh.hi.sc } \\
\text { sh.hi.sc:Bird.exc1 } \\
\text { sh.hi.sc:Bat.exc1 }\end{array}$ & $\begin{array}{r}\text { Estimate } \\
3.36925 \\
-0.08088 \\
0.52790 \\
-0.20471 \\
0.22533 \\
-0.99461 \\
-0.03288 \\
-0.02249 \\
-0.11577 \\
0.03975 \\
0.01521\end{array}$ & $\begin{array}{r}\text { std. Error } \\
0.14273 \\
0.10665 \\
0.26130 \\
0.10900 \\
0.11205 \\
0.24172 \\
0.11007 \\
0.06938 \\
0.11297 \\
0.11122 \\
0.11117\end{array}$ & $\begin{array}{r}\text { z value } \\
23.605 \\
-0.758 \\
2.020 \\
-1.878 \\
2.011 \\
-4.115 \\
-0.299 \\
-0.324 \\
-1.025 \\
0.357 \\
0.137\end{array}$ & $\begin{array}{r}\operatorname{Pr}(>|z|) \\
<2 \mathrm{e}-16 \\
0.4482 \\
0.0434 \\
0.0604 \\
0.0443 \\
3.88 \mathrm{e}-05 \\
0.7651 \\
0.7458 \\
0.3055 \\
0.7208 \\
0.8912\end{array}$ & $\begin{array}{l}* \\
* \\
* * *\end{array}$ \\
\hline
\end{tabular}




\section{Table S13.11 Ants}

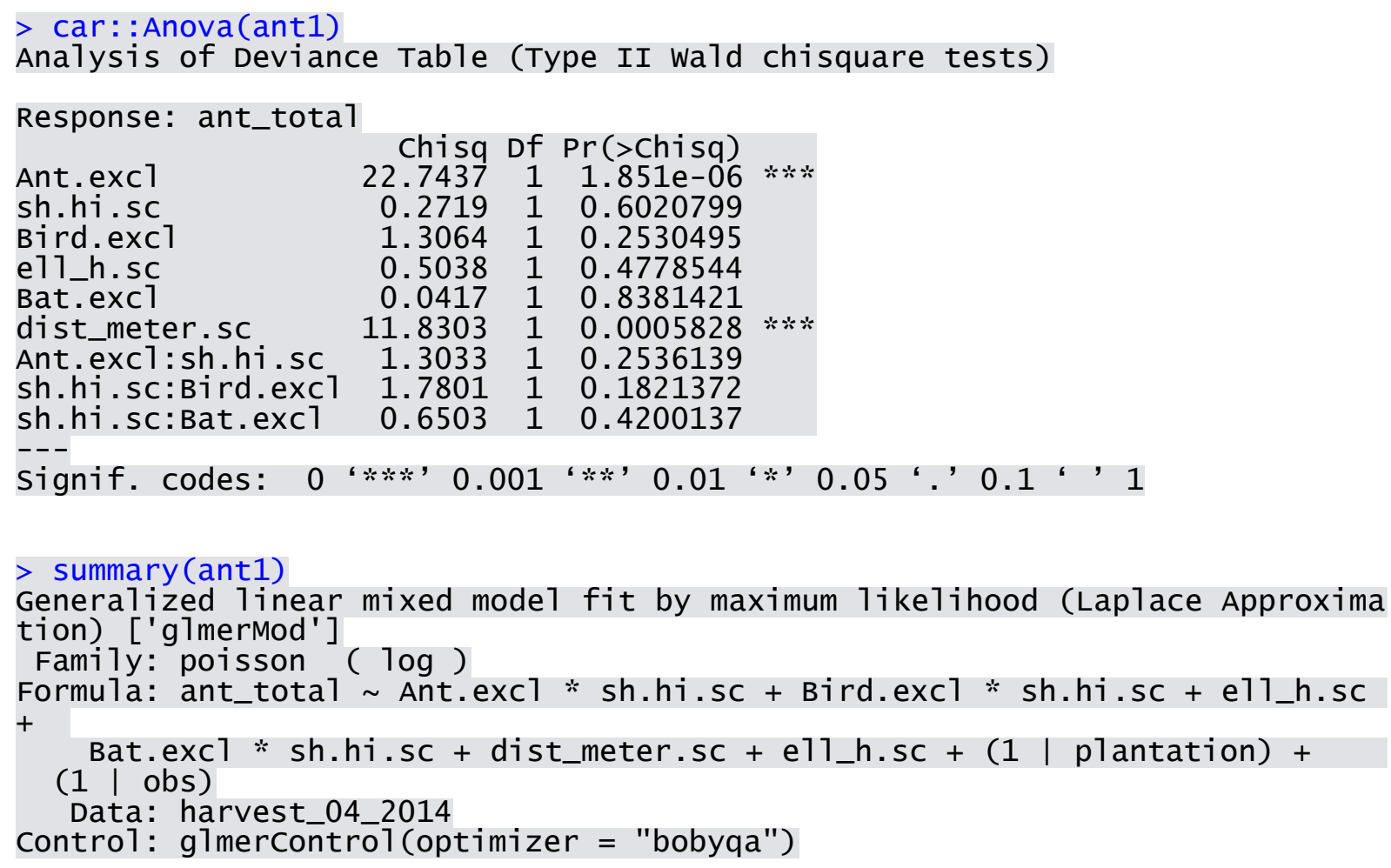

\begin{tabular}{|c|c|c|c|c|c|}
\hline $\begin{array}{l}\text { (Intercept) } \\
\text { Ant.exc1 } \\
\text { sh.hi.sc } \\
\text { Bird.exc1 } \\
\text { e11_h.sc } \\
\text { Bat.exc1 } \\
\text { dist_meter.sc } \\
\text { Ant.excl:sh.hi.sc } \\
\text { sh.hi.sc:Bird.exc1 } \\
\text { sh.hi.sc:Bat.excl }\end{array}$ & $\begin{array}{r}\text { Estimate } \\
4.60181 \\
-1.13614 \\
0.00774 \\
0.28084 \\
0.10100 \\
-0.05397 \\
0.50521 \\
0.27089 \\
-0.31930 \\
0.19342\end{array}$ & $\begin{aligned} \text { Std. Error } \\
0.25016 \\
0.23772 \\
0.24244 \\
0.24177 \\
0.14230 \\
0.24782 \\
0.14688 \\
0.23729 \\
0.23932 \\
0.23986\end{aligned}$ & $\begin{array}{r}\text { z value } \\
18.396 \\
-4.779 \\
0.032 \\
1.162 \\
0.710 \\
-0.218 \\
3.440 \\
1.142 \\
-1.334 \\
0.806\end{array}$ & $\begin{array}{l}\operatorname{Pr}(>|z|) \\
<2 \mathrm{e}-16 \\
1.76 \mathrm{e}-06 \\
0.974533 \\
0.245401 \\
0.477854 \\
0.827607 \\
0.000583 \\
0.253614 \\
0.182137 \\
0.420014\end{array}$ & $\begin{array}{l}* * * \\
* * *\end{array}$ \\
\hline
\end{tabular}




\section{Table S13.12 Web-spiders $<4 \mathrm{~mm}$}

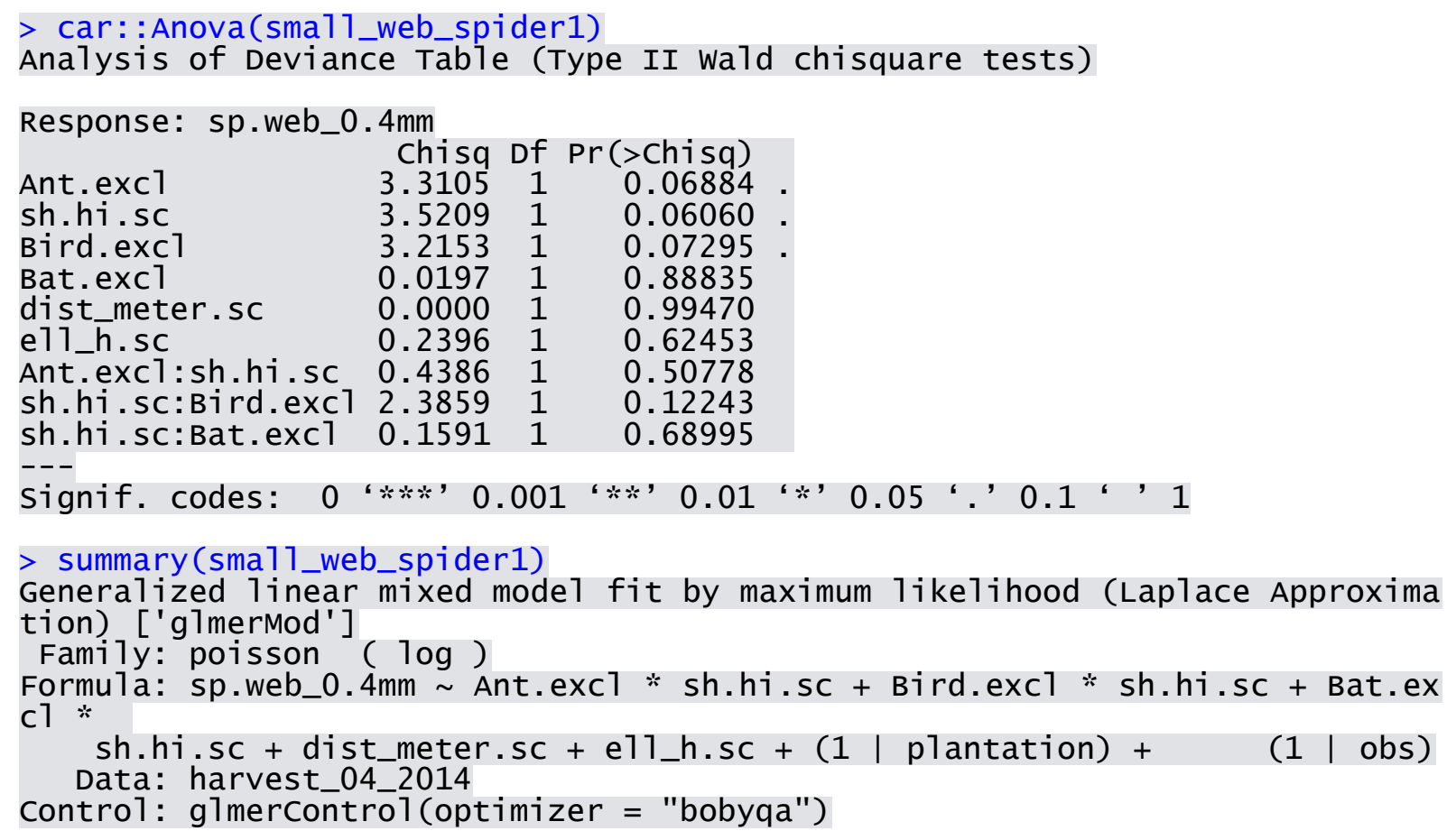

$\begin{array}{lrrrr} & \text { Estimate } & \text { Std. Error } & \text { value } & \operatorname{Pr}(>|z|) \\ \text { (Intercept) } & 1.794300 & 0.217497 & 8.250 & <2 \mathrm{e}-16 \\ \text { Ant.exc1 } & 0.234556 & 0.126486 & 1.854 & 0.0637 \\ \text { sh.hi.sc } & -0.048639 & 0.151235 & -0.322 & 0.7477 \\ \text { Bird.excl } & 0.239042 & 0.128472 & 1.861 & 0.0628 \\ \text { Bat.exc1 } & -0.020935 & 0.134008 & -0.156 & 0.8759 \\ \text { dist_meter.sc } & -0.001254 & 0.188731 & -0.007 & 0.9947 \\ \text { e11_h.sc } & -0.043703 & 0.089291 & -0.489 & 0.6245 \\ \text { Ant.exc1:sh.hi.sc } & -0.084699 & 0.127886 & -0.662 & 0.5078 \\ \text { sh.hi.sc:Bird.exc1 } & -0.197519 & 0.127873 & -1.545 & 0.1224 \\ \text { sh.hi.sc:Bat.exc1 } & 0.050875 & 0.127532 & 0.399 & 0.6900\end{array}$




\section{Table S13.13 Web-Spiders 4-10mm}

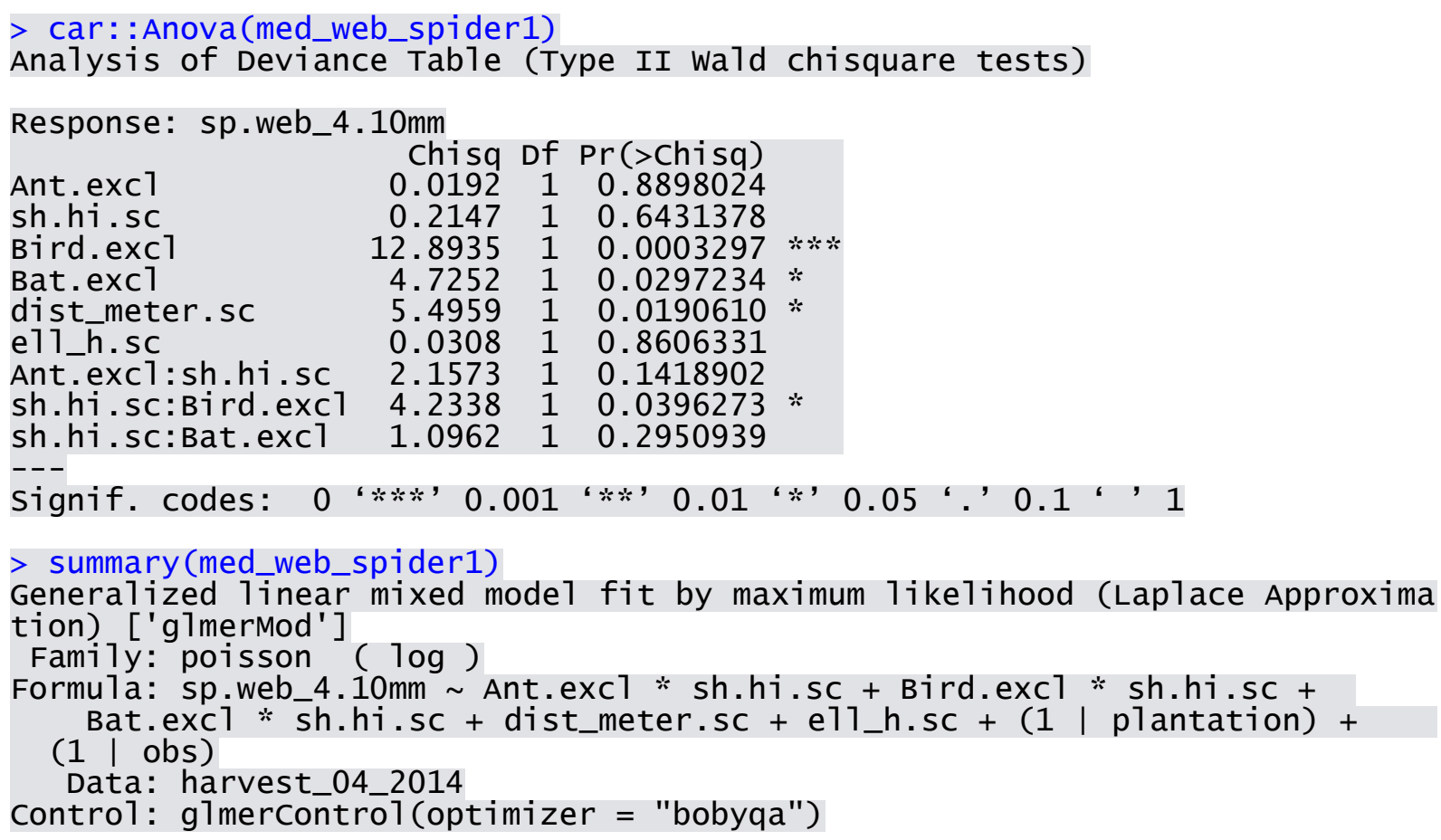

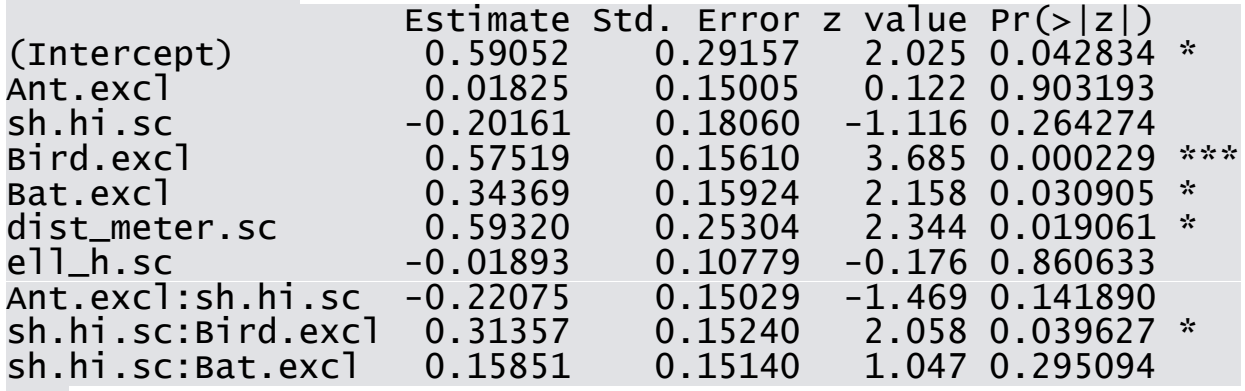




\section{Table S13.14 Dermaptera}

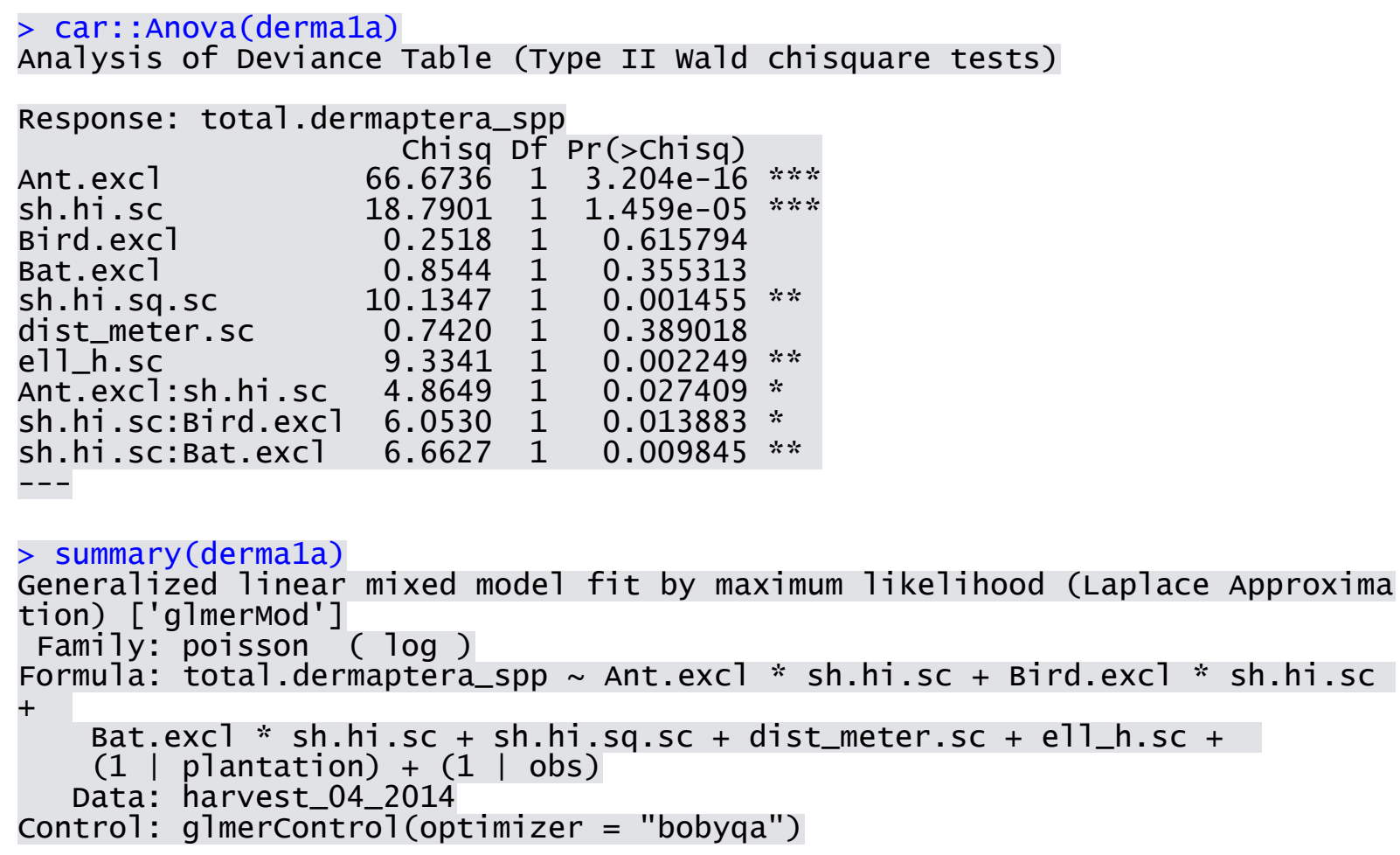

$\begin{array}{lrrrrr} & \text { Estimate } & \text { Std. Error } & \text { value } & \operatorname{Pr}(>|z|) & * * \\ \text { (Intercept) } & 1.40983 & 0.30013 & 4.697 & 2.63 \mathrm{e}-06 & * * * \\ \text { Ant.exc1 } & -1.54878 & 0.18419 & -8.408 & <2 \mathrm{e}-16 & * * * \\ \text { sh.hi.sc } & 2.10088 & 0.53942 & 3.895 & 9.83 \mathrm{e}-05 & * * * * \\ \text { Bird.exc1 } & 0.21609 & 0.17743 & 1.218 & 0.22327 & \\ \text { Bat.exc1 } & 0.02741 & 0.17866 & 0.153 & 0.87808 & \\ \text { sh.hi.sq.sc } & -1.43176 & 0.44974 & -3.183 & 0.00146 * * \\ \text { dist_meter.sc } & 0.22540 & 0.26166 & 0.861 & 0.38902 & \\ \text { e11_h.sc } & 0.35325 & 0.11562 & 3.055 & 0.00225 * * \\ \text { Ant.exc1:sh.hi.sc } & 0.46485 & 0.21075 & 2.206 & 0.02741 * \\ \text { sh.hi.sc:Bird.exc1 } & -0.48305 & 0.19634 & -2.460 & 0.01388 * \\ \text { sh.hi.sc:Bat.exc1 } & 0.52208 & 0.20226 & 2.581 & 0.00985 * *\end{array}$




\section{Table S13.15 Coleoptera $<4 \mathrm{~mm}$}

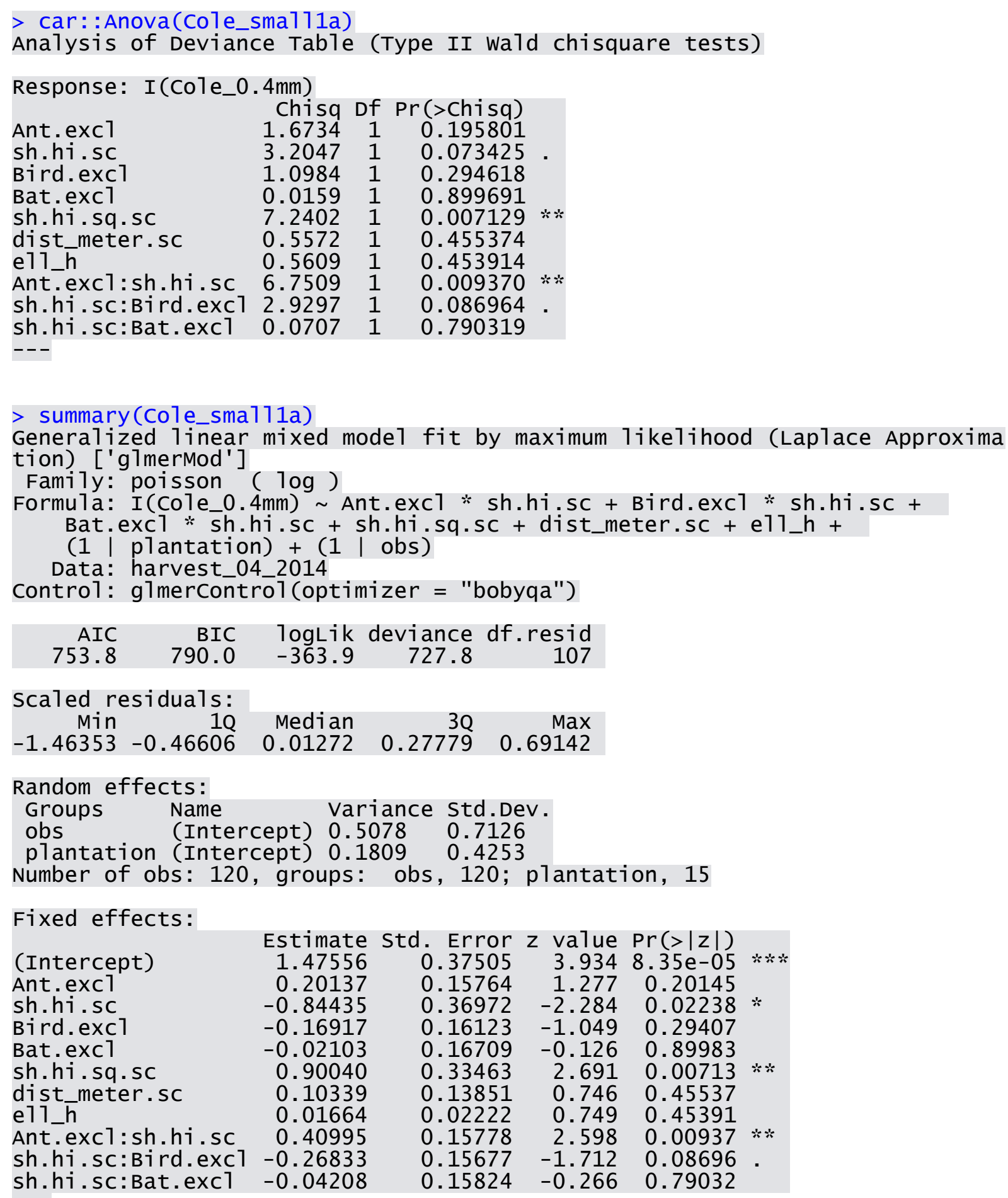




\section{Table S13.16 Leaf herbivory}
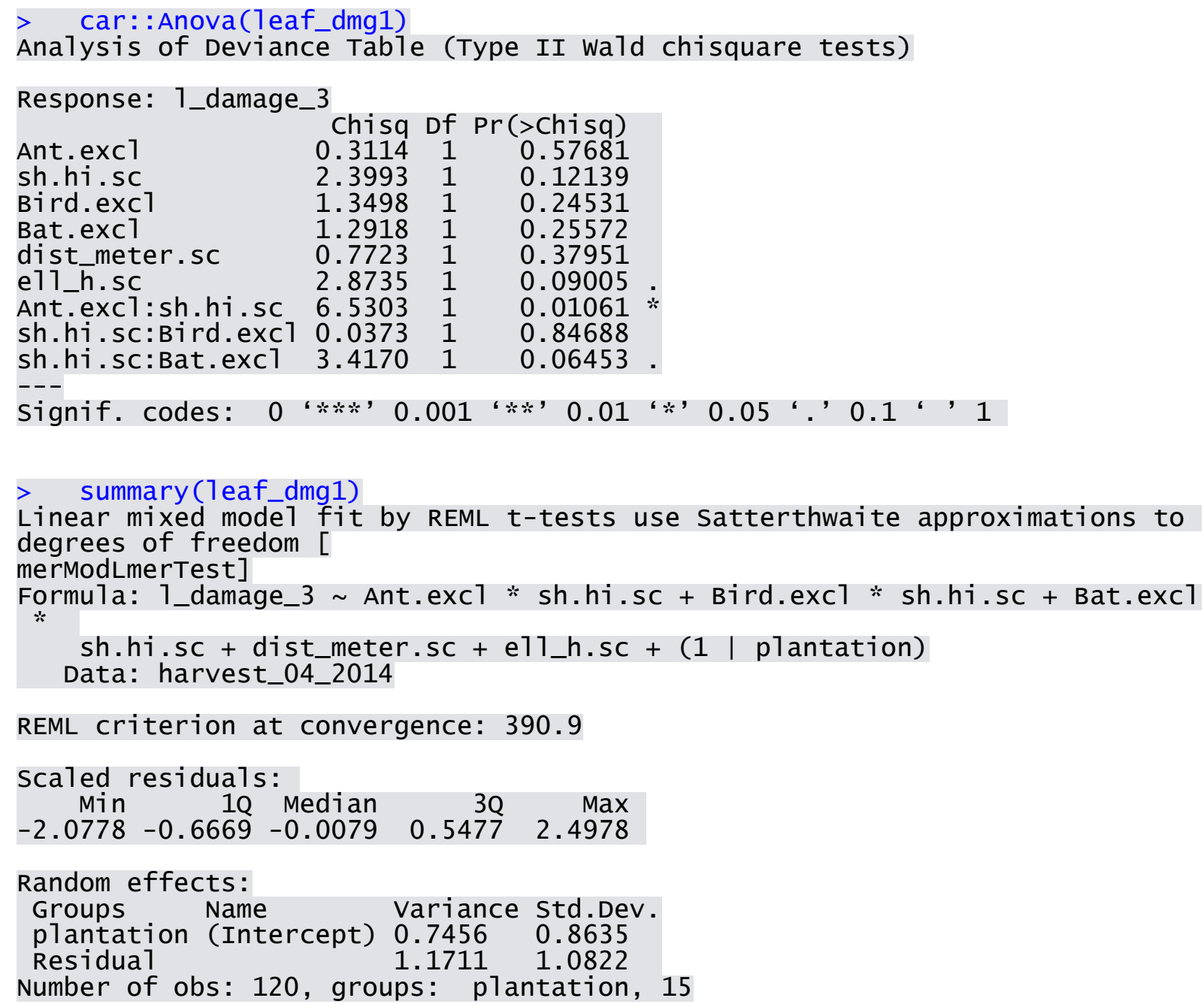

Fixed effects:

\begin{tabular}{|c|c|c|c|c|c|}
\hline $\begin{array}{l}\text { (Intercept) } \\
\text { Ant.exc1 } \\
\text { sh.hi.sc } \\
\text { Bird.exc1 } \\
\text { Bat.excl } \\
\text { dist_meter.sc } \\
\text { el1_h.sc } \\
\text { Ant.excl:sh.hi.sc } \\
\text { sh.hi.sc:Bird.exc1 } \\
\text { sh.hi.sc:Bat.exc1 }\end{array}$ & $\begin{array}{r}\text { Estimate } \\
2.73917 \\
0.12179 \\
0.72401 \\
0.24157 \\
0.24713 \\
0.21788 \\
-0.22987 \\
-0.52087 \\
-0.03949 \\
-0.37966\end{array}$ & $\begin{array}{r}\text { Std. Error } \\
0.30229 \\
0.20293 \\
0.22990 \\
0.20794 \\
0.21362 \\
0.24793 \\
0.13561 \\
0.20383 \\
0.20450 \\
0.20539\end{array}$ & $\begin{array}{r}\mathrm{df} \\
26.75000 \\
97.23000 \\
109.99000 \\
98.04000 \\
98.85000 \\
12.29000 \\
109.07000 \\
98.15000 \\
97.59000 \\
98.13000\end{array}$ & $\begin{array}{r}\text { t value } \\
9.061 \\
0.600 \\
3.149 \\
1.162 \\
1.157 \\
0.879 \\
-1.695 \\
-2.555 \\
-0.193 \\
-1.849\end{array}$ & $\begin{array}{l}\operatorname{Pr}(>|t|) \\
1.23 \mathrm{e} \mid 09 \\
0.54979 \\
0.00211 \\
0.24816 \\
0.25011 \\
0.39637 \\
0.09290 \\
0.01214 \\
0.84728 \\
0.06754\end{array}$ \\
\hline
\end{tabular}




\section{Table S13.17 Diptera $<4 \mathrm{~mm}$}

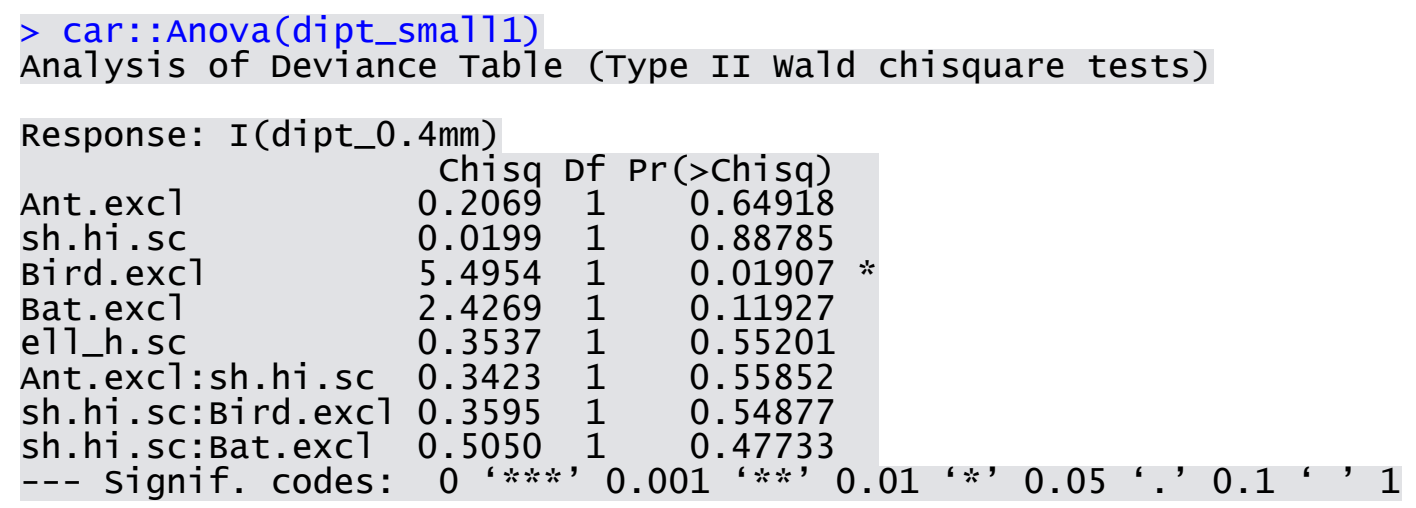

> summary(dipt_sma111)

Generalized Tinear mixed model fit by maximum likelihood (Laplace Approxima tion) ['g7merMod']

Family: poisson ( $10 \mathrm{~g}$ )

Formula: I(dipt_0.4mm) $\sim$ Ant.excl $*$ sh.hi.sc + Bird.excl $*$ sh.hi.sc + Bat.excl *sh.hi.sc + e11_h.sc + (1 | plantation $)+(1 \mid$ obs $)$

Data: harvest_04_2014

Contro1: g7merControl(optimizer = "bobyqa")

$\begin{array}{rrrrr}\text { AIC } & \text { BIC } & \text { logLik deviance } & \text { df.resid } \\ 1037.3 & 1068.0 & -507.6 & 1015.3 & 109\end{array}$

Scaled residuals:

$\begin{array}{rrrrr}\text { Min } & 1 \mathrm{Q} & \text { Median } & 3 \mathrm{Q} & \text { Max } \\ -1.89456 & -0.22411 & 0.00548 & 0.19967 & 0.61684\end{array}$

Random effects:

$\begin{array}{llll}\text { Groups } & \text { Name } & \text { Variance } & \text { Std.Dev. } \\ \text { obs } & \text { (Intercept) } & 0.2775 & 0.5268 \\ \text { plantation (Intercept) } & 0.2758 & 0.5252 \\ \text { Number of obs: 120, groups: obs, } 120 ; \text { plantation, } 15\end{array}$

Fixed effects:

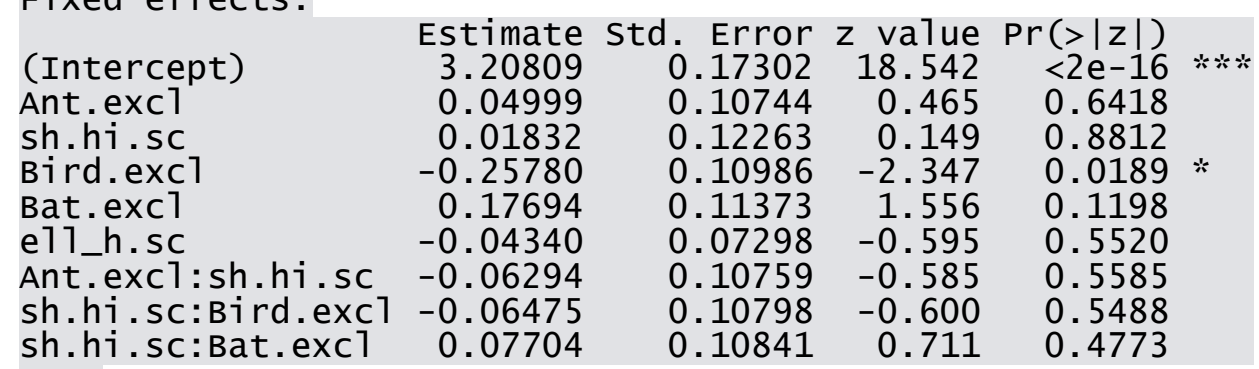



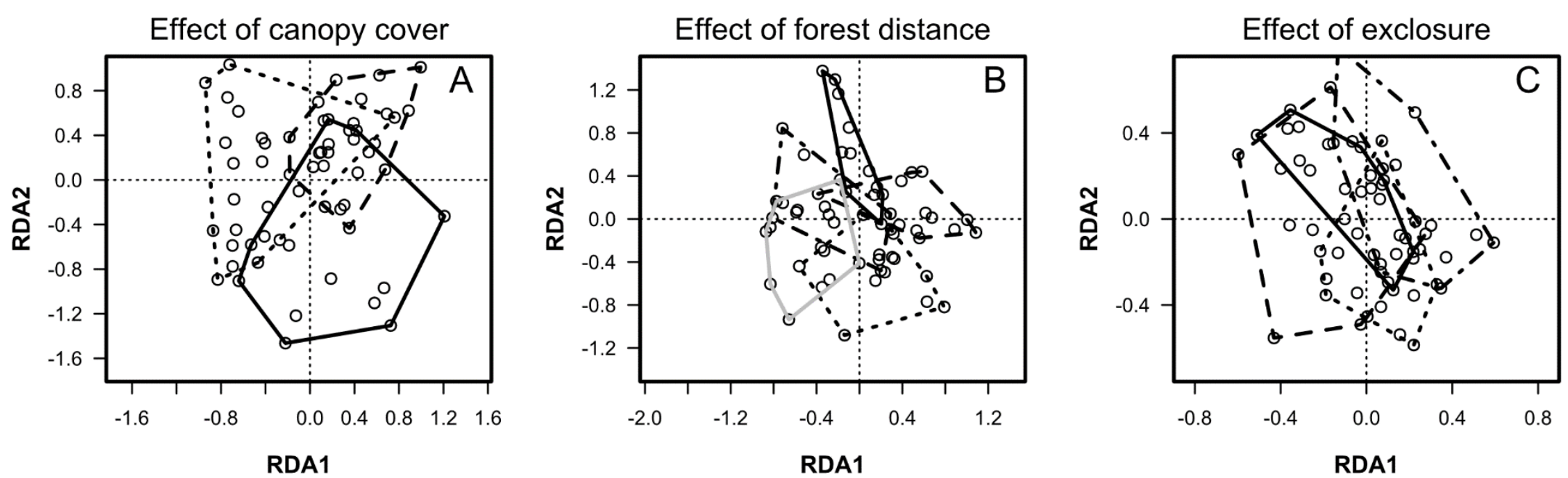

$\stackrel{\infty}{2}$

Fig. S14. RDA - Management, landscape, and predator access manipulation influences on the ant community composition (morpho species level).

Figure A: canopy cover: continuous line $=>50 \%$ canopy cover, dashed line $=30-50 \%$ canopy cover, dotted line $=<30 \%$ canopy cover; figure B: forest distance: black continuous line $=0-250 \mathrm{~m}$, black dashed line $=251-500 \mathrm{~m}$, black dash-dotted line $=501-1500 \mathrm{~m}$, black dotted line $=1501-2500$ $\mathrm{m}$, grey-continuous line $=2500-3000 \mathrm{~m}$; figure C: treatments: black $=$ control, dotted line $=$ bird exclusion, dashed line $=$ bat exclusion, dot-dashed line $=$ bird and bat exclusion. 

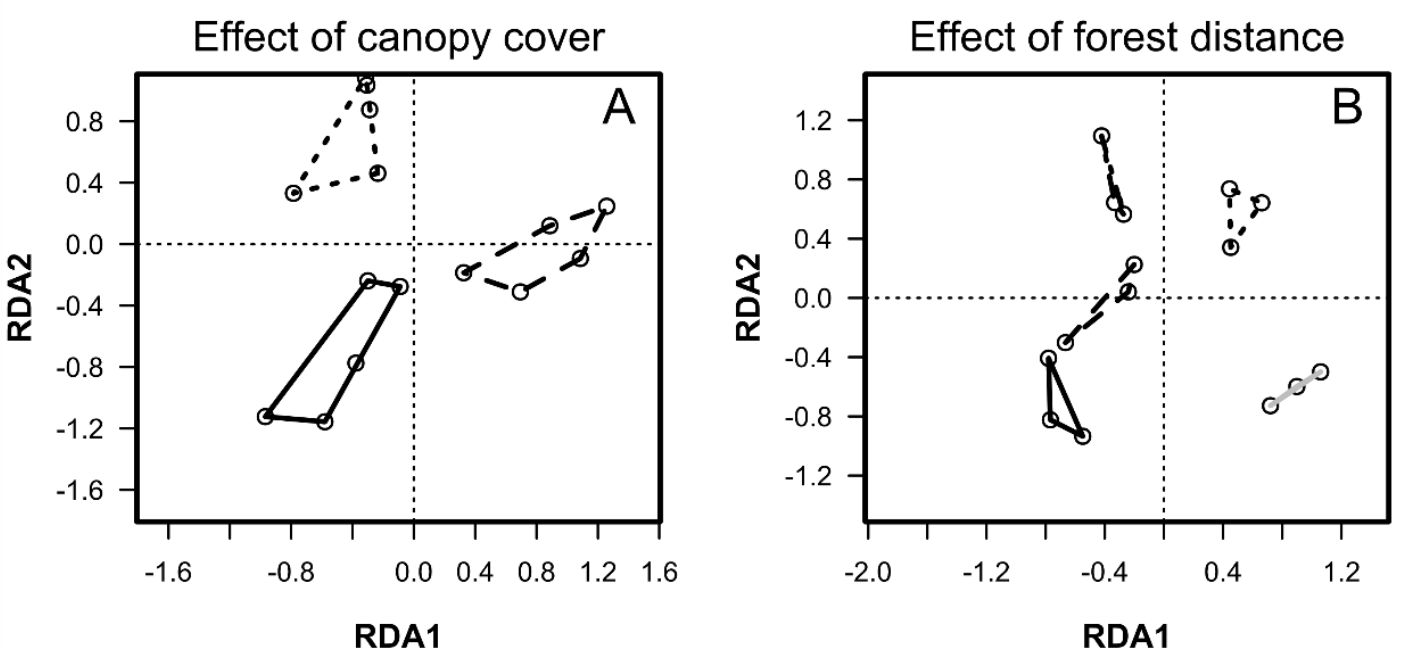

Fig. S15. RDA - Management and landscape effects on the bird community (species level).

Figure A: canopy cover: continuous line $=>50 \%$ canopy cover, dashed line $=30-50 \%$ canopy cover, dotted line $=<30 \%$ canopy cover; Figure B: forest distance: black continuous line $=0-250 \mathrm{~m}$, black dashed line $=251-500 \mathrm{~m}$, black dash-dotted line $=501-1500 \mathrm{~m}$, black dotted line $=1501-2500 \mathrm{~m}$, grey-continuous line $=2500-3000 \mathrm{~m}$. 
Fig. S16. Visualization of bird and ant community composition related to shade cover and forest distance.

Data see Table S6. Red circles (birds) and blue dots (ants) indicate site specific diversity estimators of rarefied species richness, Shannon Index and Species evenness. Bird data was collected via mist-netting and point counts and the ant diversity was recorded using bait-trapping at 15 cacao plantations in Central Sulawesi, Indonesia.
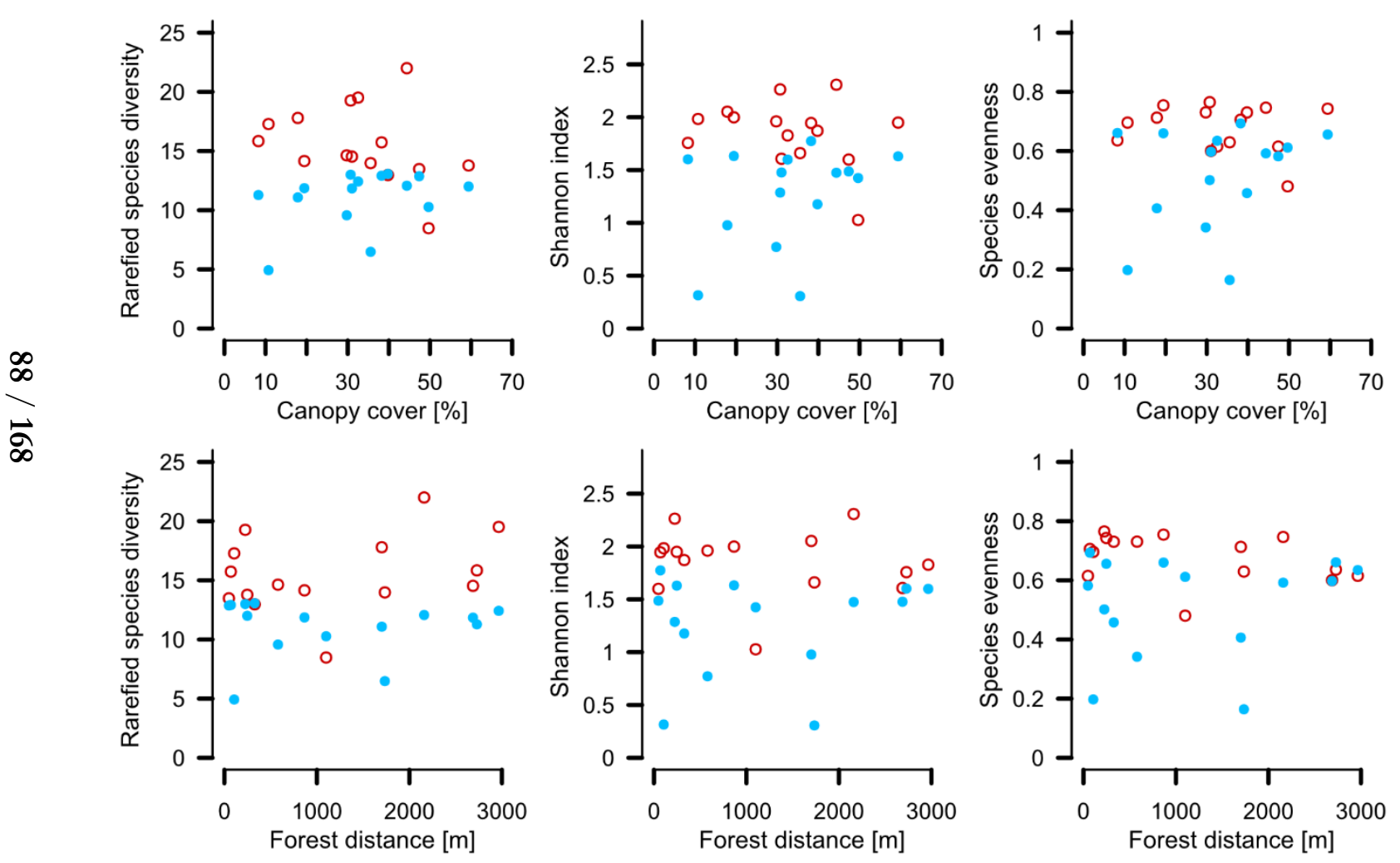

- Birds
- Ants 


\section{Table S17. Bat species}

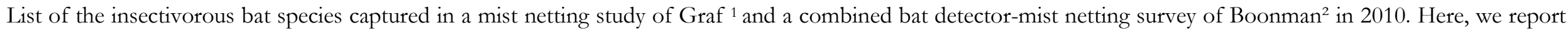

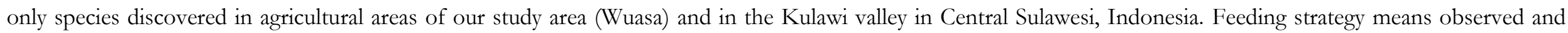
reported behaviour reported data bases or was inferred from reported habitat, flying behaviour and prey: approximations are italic and superscripts indicates the source.

Variation in feeding behaviour of bats is common. The reported strategy seems likely, but other feeding strategies cannot be excluded.

\begin{tabular}{|c|c|c|c|c|c|c|c|c|c|}
\hline $\begin{array}{l}\text { Feeding } \\
\text { strategy }\end{array}$ & Obs. & Loc. & Family & Species & Weight (g) & FA (mm) & Roosting & Foraging space & Food items \\
\hline $\begin{array}{l}\text { Aerial-hawking / } \\
\text { Hovering } 2^{2}\end{array}$ & B & W & Vespertilionidae & $\begin{array}{ll}\text { Harphiocephalus } & \text { barpia } \\
\text { (Temminck, 1840) } & \end{array}$ & ca. 12.4 & $44.1-50.1$ & NA & $\begin{array}{l}\text { Fields, villages, } \\
\text { plantation, ponds }\end{array}$ & Coleoptera \\
\hline Aerial_hawking 1,2 & B / G & $\mathrm{W} / \mathrm{K}$ & Verspertilionidae & $\begin{array}{l}\text { Myotis } \\
\text { (Gray, 1864) }\end{array}$ & $3.9-5.5$ & $32.3-36.4$ & $\begin{array}{l}\text { Rolled banana leaves, } \\
\text { caves, tree hollows }\end{array}$ & $\begin{array}{l}\text { Agricultural landscape, } \\
\text { along streams, roads in } \\
\text { open and semi-open } \\
\text { places, several metres } \\
\text { above ground level }\end{array}$ & Insects \\
\hline Aerial-bawking 1,2 & B & W & Verspertilionidae & $\begin{array}{l}\text { Scotophilus } \\
\text { (Leach, 1821) }\end{array}$ & $16.8-24.9$ & $44.0-56.4$ & $\begin{array}{l}\text { Temples, caves, } \\
\text { hollow trees, palm } \\
\text { fronds, roofs, old } \\
\text { houses, dry leaves of } \\
\text { trees }\end{array}$ & $\begin{array}{l}\text { Agricultural areas, } \\
\text { villages, primary and } \\
\text { secondary habitats }\end{array}$ & Hymenoptera, Diptera \\
\hline $\begin{array}{l}\text { Aerial-hawking/ } \\
\text { hovering } \\
\text { perching } 1,4\end{array}$ & B / G & $\mathrm{W} / \mathrm{K}$ & Hipposideridae & $\begin{array}{l}\text { Hipposideros } \\
\text { (Gould, 1863) }\end{array}$ & $5.6-9.0$ & $45.0-47.0$ & $\begin{array}{l}\text { Caves, abandoned } \\
\text { mines, occasionally } \\
\text { sheds and buildings }\end{array}$ & $\begin{array}{l}\text { Between trees and } \\
\text { shrubs, rainforest, } \\
\text { gallery forest, open } \\
\text { savannah woodland. }\end{array}$ & $\begin{array}{l}\text { Medium-sized airborne } \\
\text { insects (i.e. Lepidoptera } \\
\text { and Coleoptera) }\end{array}$ \\
\hline $\begin{array}{l}\text { Aerial-hawking } \\
\text { hovering } 1,2\end{array}$ & B & W & Emballonuridae & $\begin{array}{l}\text { Taphozous saccolaimus } \\
\text { (Temminck, 1838) }\end{array}$ & $31.0-60.0$ & $63.0-75.0$ & $\begin{array}{l}\text { Hollows of old and } \\
\text { decaying trees, old } \\
\text { buildings,rocky } \\
\text { crevices }\end{array}$ & $\begin{array}{l}\text { Agricultural areas, } \\
\text { villages, all vertical } \\
\text { strata }\end{array}$ & $\begin{array}{l}\text { Isoptera, Coleoptera, } \\
\text { other insects }\end{array}$ \\
\hline Hovering ${ }^{1}$ & B & W & Verspertilionidae & $\begin{array}{l}\text { Hypsugo imbricatus } \\
\text { (Horsfield, 1824) }\end{array}$ & NA & $32.5-36.0$ & Buildings, trees & $\begin{array}{l}\text { Plantations, gardens, } \\
\text { bamboo areas }\end{array}$ & Insects \\
\hline Hovering $1,2,3$ & B & W & Verspertilionidae & $\begin{array}{l}\text { Kerivoula } \\
\text { (i.e. hardwickii) }\end{array}$ & $3.1-4.2$ & $<30.1$ & Buildings, trees & $\begin{array}{l}\text { Plantations, vilages, } \\
\text { between trees and } \\
\text { shrubs }\end{array}$ & Insects \\
\hline
\end{tabular}




\begin{tabular}{|c|c|c|c|c|c|c|c|c|c|}
\hline $\begin{array}{l}\text { Hovering } \\
\text { perching }{ }^{2}\end{array}$ & G & $\mathrm{K}$ & Megadermatidae & $\begin{array}{l}\text { Megaderma } \\
\text { (Linnaeus, 1758) }\end{array}$ & $13.0-28.0$ & $54.0-63.0$ & $\begin{array}{l}\text { Empty buildings, } \\
\text { Caves }\end{array}$ & $\begin{array}{l}\text { Between trees and } \\
\text { shrubs, rocky spaces }\end{array}$ & $\begin{array}{l}\text { Orthoptera, } \\
\text { Lepidoptera, Coleoptera, } \\
\text { large flying insects }\end{array}$ \\
\hline Perching ${ }^{2}$ & G & $\mathrm{K}$ & Rhinolophidae & $\begin{array}{l}\text { Rbinolophus } \\
\text { (Peters, 1861) }\end{array}$ & $8.0-10.9$ & $41.0-47.0$ & $\begin{array}{l}\text { Hollow bamboos, } \\
\text { bananas leaves , } \\
\text { hollow trees, rock } \\
\text { crevices, caves }\end{array}$ & $\begin{array}{l}\text { Understory of } \\
\text { primary and secondary } \\
\text { forest }\end{array}$ & Insects \\
\hline Perching ${ }^{3}$ & B & W & Hipposideridae & $\begin{array}{l}\text { Hipposideros diadema } \\
\text { (É. Geoffroy, 1813) }\end{array}$ & $33.0-45.0$ & $74.0-96.0$ & Caves, hollow trees & $\begin{array}{l}\text { Hanging from twigs, } \\
\text { woodlands }\end{array}$ & $\begin{array}{l}\text { Coleoptera, Lepidoptera, } \\
\text { Orthoptera, rarely small } \\
\text { birds and spiders }\end{array}$ \\
\hline $\begin{array}{l}\text { Surface gleaning / } \\
\text { Aerial-bawking } 2\end{array}$ & B / G & $\mathrm{W} / \mathrm{K}$ & Verspertilionidae & Myotis ater (Peters, 1866) & $4.4-5.1$ & $35.0-37.1$ & Village areas & $\begin{array}{l}\text { Agricultural areas, } \\
\text { villages }\end{array}$ & Insects \\
\hline $\begin{array}{l}\text { Surface gleaning }{ }^{2} \\
\text { (e.g. water, leaves) }\end{array}$ & G & $\mathrm{K}$ & Verspertilionidae & $\begin{array}{l}\text { Myotis borsfieldii } \\
\text { (Temminck, 1840) }\end{array}$ & $5.0-12.5$ & $33.0-36.8$ & $\begin{array}{l}\text { Caves, tunnels, } \\
\text { bridges, palms, old } \\
\text { buildings }\end{array}$ & $\begin{array}{l}\text { Agricultural areas, } \\
\text { primary and secondary } \\
\text { forests }\end{array}$ & Insects \\
\hline NA 1,2 & B & W & Emballonuridae & $\begin{array}{l}\text { Mosia } \\
\text { (Gray, 1843) }\end{array}$ & NA & NA & $\begin{array}{l}\text { Palm leaves, caves, } \\
\text { rock overhangs, } \\
\text { house roofs }\end{array}$ & $\begin{array}{l}\text { Primary and secondary } \\
\text { habitats, plantations, } \\
\text { gardens, villages }\end{array}$ & Insects \\
\hline $\mathrm{NA}^{1,2}$ & B & W & Rhinolophidae & $\begin{array}{l}\text { Rbinolophus celebensis } \\
\text { (Anderson, 1905) }\end{array}$ & $<7.5$ & $38.0-45.0$ & Cave & $\begin{array}{l}\text { Primary and secondary } \\
\text { forest }\end{array}$ & Insects \\
\hline $\mathrm{NA}^{1,2}$ & B / G & $\mathrm{W} / \mathrm{K}$ & Rhinolophidae & $\begin{array}{l}\text { Rbinolophus euryotis } \\
\text { (Temminck, 1835) }\end{array}$ & ca. 21.5 & $54.0-73.0$ & $\begin{array}{l}\text { Caves, old mining } \\
\text { shafts, tunnels }\end{array}$ & NA & Insects \\
\hline $\mathrm{NA}^{1,2}$ & $\mathrm{~B}$ & W & Verspertilionidae & $\begin{array}{ll}\text { Scotophilus } & \text { celebensis } \\
\text { (Simmons 2005) }\end{array}$ & NA & NA & $\begin{array}{l}\text { Caves, } \\
\text { houses }\end{array}$ & $\begin{array}{l}\text { Open areas, above the } \\
\text { canopy. }\end{array}$ & $\begin{array}{l}\text { Airborne insects, } \\
\text { Hymenoptera, } \\
\text { Diptera,Lepidoptera, } \\
\text { Coleoptera }\end{array}$ \\
\hline
\end{tabular}

${ }^{1}$ Graf, S. (2010). Diversity and habitat use of understorey bats in forest and agroforestry systems at the margin of Lore Lindu National Park (Central Sulawesi, Indonesia). Diploma thesis, University of Vienna. (The study was conducted in the Kulawi valley, situated at the western border of the Lore Lindu National Park in Central Sulawesi, approximately $23 \mathrm{~km}$ apart from our study area in Napu valley. Insectivorous bat species (Microchiroptera) were captured along a babitat gradient (natural forests, selectively logged forests and agroforestry systems) and belonged to 4 families and 7 species.).

${ }^{2}$ Boonman, A. (2010). Report of an unpublished bat survey in Central Sulawesi using mist netting and bat detectors. Bats (Microchiroptera) were surveyed around the Lore Lindu national Park at 9 different sites. Here we report species discovered at sites in our study region (Wuasa, 4 sites) and the Kulawi valley (1 site) 


\section{Table S18 Agroforest description}

Plantation: site identity, age $=$ age of cacao trees in 2011, avg. tree height $=$ mean height of all 16 experimental trees in one agroforest, size $=$ area of one plantation, number of cacao trees $=$ estimated tree abundance, land use system before $=$ what was there before planting cacao, land use systems around $=$ crops in proximity, slope $=$ slope inclination in degree, last 5 columns indicate use of drainage or chemicals $(\mathrm{y}=$ yes used, $\mathrm{n}=\mathrm{not}$ used).

\begin{tabular}{|c|c|c|c|c|c|c|c|c|c|c|c|c|c|}
\hline 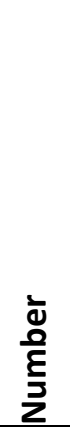 & $\begin{array}{l}\frac{5}{0} \\
\stackrel{0}{\pi} \\
\stackrel{0}{\frac{1}{2}} \\
\frac{\pi}{\alpha}\end{array}$ & 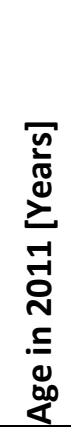 & 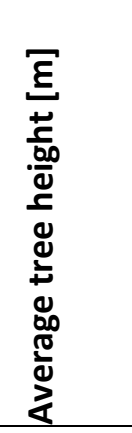 & 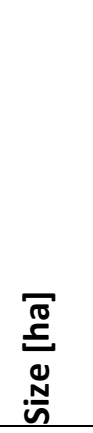 & 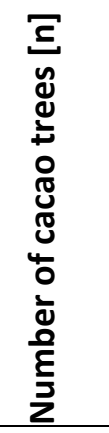 & 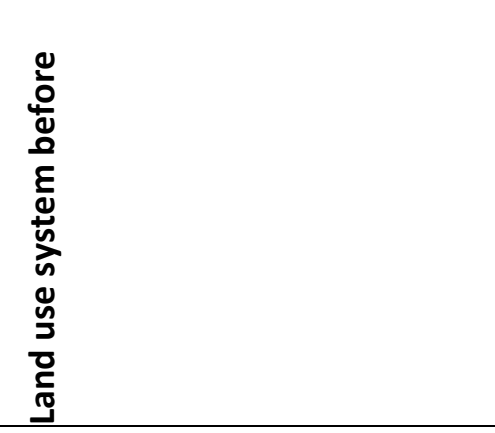 & 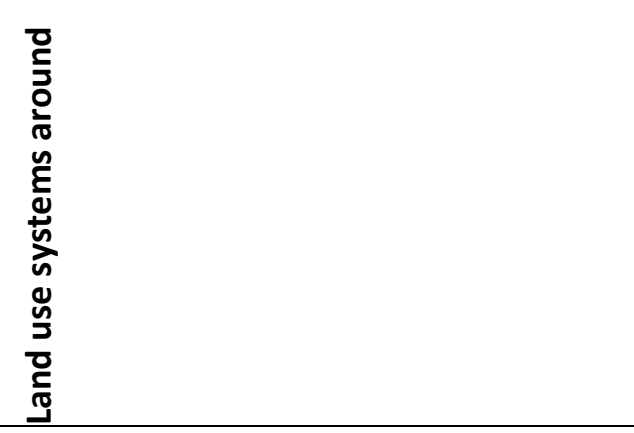 & $\begin{array}{l}\frac{\sigma}{\circ} \\
\frac{0}{0} \\
\frac{0}{n}\end{array}$ & 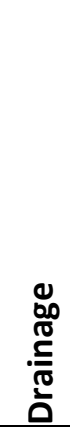 & 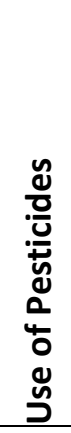 & 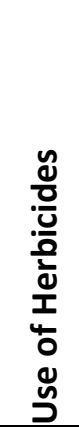 & 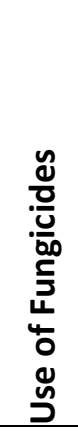 & 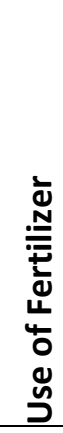 \\
\hline 2 & Banti & 9 & 331.88 & 0.5 & 400 & maize & maize & 0 & $\mathrm{n}$ & $\mathrm{n}$ & y & $\mathrm{n}$ & $\mathrm{n}$ \\
\hline 3 & Bolai & 6 & 346.25 & 0.8 & 500 & forest & cacao, tomato & 0 & $\mathrm{n}$ & $\mathrm{n}$ & y & $\mathrm{n}$ & $\mathrm{n}$ \\
\hline 4 & Ciko & 7 & 324.38 & 1 & 650 & maize & cacao & 0 & $\mathrm{n}$ & $y$ & y & $\mathrm{n}$ & $\mathrm{n}$ \\
\hline 5 & Dedi & 12 & 392.13 & 2 & 1300 & coffee, vanilla & cacao, vanilla, orange & 0 & $\mathrm{n}$ & $\mathrm{n}$ & y & $\mathrm{n}$ & $\mathrm{n}$ \\
\hline 6 & Deni & 4 & 255 & 0.75 & 575 & maize & cacao, vegetables, maize & 0 & $\mathrm{n}$ & $\mathrm{n}$ & y & $\mathrm{n}$ & y \\
\hline 7 & Dewa & 5 & 309.38 & 1.25 & 1000 & vegetables (Tomato, cabbage) & cacao, vegetables & 0 & $\mathrm{n}$ & y & y & $\mathrm{n}$ & $\mathrm{n}$ \\
\hline 12 & Main & 5 & 274.38 & 3 & 2400 & maize & cacao, vegetables, maize, chilli, banana & 0 & $\mathrm{n}$ & $y$ & y & $y$ & y \\
\hline 13 & Ponedi & 6 & 253.13 & 0.75 & 560 & maize & cacao, coffee, vagetable, onion & 0 & y & $\mathrm{n}$ & y & $\mathrm{n}$ & $\mathrm{n}$ \\
\hline 14 & Roby & 7 & 271.25 & 0.5 & 200 & maize & maize & 0 & $\mathrm{n}$ & $y$ & $y$ & $\mathrm{n}$ & $y$ \\
\hline 15 & Toni & 7 & 313.75 & 1 & 400 & vegetables & cacao, vagetables, maize & 0 & $\mathrm{n}$ & $\mathrm{y}$ & $y$ & $\mathrm{n}$ & $y$ \\
\hline
\end{tabular}


$92 / 168$ 


\section{Chapter 3: Resource distribution changes species-specific trophic}

positions and ecosystem service provision of omnivorous ants

Pierre Gras, Teja Tscharntke, Christoph Scherber, Aijen Tjoa, Alfianus Rumede, Yann Clough

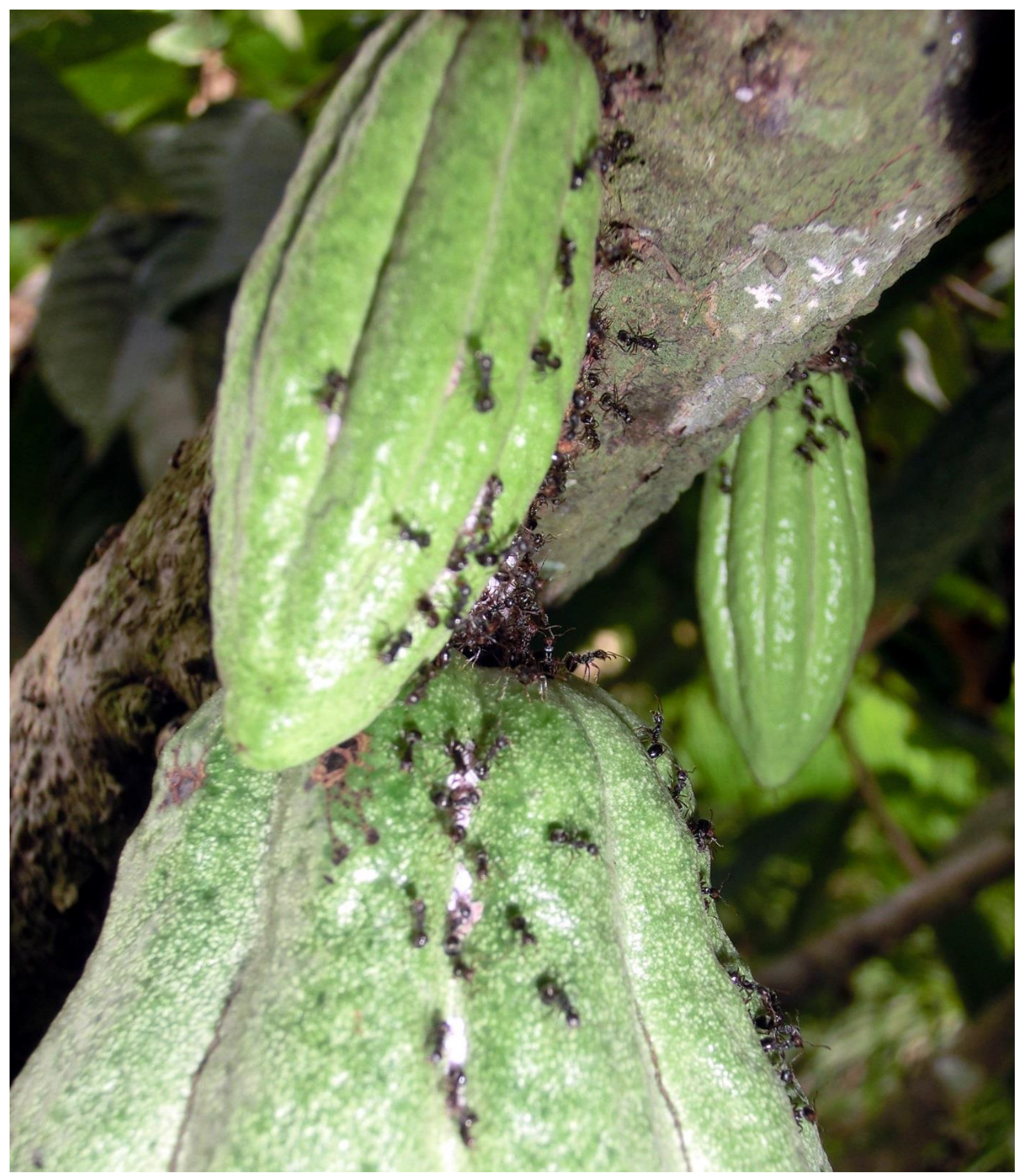

Dolichoderus thoracicus (SMITH, 1860) obtains honeydew of trophobionts (Cataenococcus hispidus Morrison (Hemiptera: Pseudococcidae)) at small, young medium, and big sized cacao fruits. Typically, the trophobionts colonising the fruit grooves first.

Manuscript prepared for: Ecology (status: not yet submitted) 


\section{Summary}

Omnivores play a plastic and ambivalent trophic role, feeding both plant and animal based diets. Feeding strategies of omnivorous tropical ants are ecologically important, shaping arthropod and plant communities. We investigated the relationship of temporal trophic shifts and resource availability of omnivores in Indonesian cacao agroforestry. We experimentally established 32 colonies of two arboreal, omnivorous ant species in cacao agroforests. Between cacao flowering and harvest, we surveyed all plantations over five months to estimate colony size, tree phenology, prey availability and to assess natural abundance of stable nitrogen isotopes. Our study shows that density adaptation and trophic plasticity are necessary to compensate spatiotemporal patterns of resource distribution in agricultural landscapes. If food was scarce, both species integrated more honeydew in their diets and colony sizes dropped. Increasing caterpillar abundances resulted in increasing trophic positions and colony sizes, but the amount of accessible trophobionts influenced colony sizes only. The amount of accessible resources did not correlate with ants' hunting effort, which indicates that predatory services of ants are not directly driven by resource availability. Other influences such as interspecific competition or predator presence may be similarly important. Finally, trophic plasticity and nutritional stoichiometry help to explain why $D$. thoracicus provides ecosystem services preying on herbivores, but $P$. cf. cordata mainly provides disservices trough cryptic-herbivory and intra-guild predation.

\section{Introduction}

Omnivorous ants profoundly shape terrestrial ecosystems, simultaneously affecting a wide variety of ecosystem services, acting as cryptic herbivores, predators or scavengers in natural and agricultural habitats (Hölldobler and Wilson 1990, Folgarait 1998, Philpott and Armbrecht 2006, Philpott et al. 2008). The spatiotemporal distribution of ants depends on abiotic conditions, nesting site availability, competition, and availability of food resources such as arthropod prey, extrafloral nectaries, myrmecophytes, and trophobionts tended to obtain honeydew (Floren et al. 2002, Davidson et al. 2003). It has been hypothesized that ants adapt colony sizes to the amount of exploitable resources, with foraging efforts and diets depending on biotic and abiotic seasonality of the habitat (Chamberlain and Holland 2008, Rudolph and Palmer 2013, Warren and Chick 2013). Experiments manipulating food resources have shown ambivalent, species-specific responses to lacks in the availability of carbohydrates: (1) Ants either intensify foraging for protein sources, enabling maintenance of colony size and reproduction (Rudolph and Palmer 2013), or (2) ants reduce foraging for protein sources, increase protection of trophobionts in accordance with "resource imbalance" or "metabolic fuel" theory (Davidson 1997, Grover et al. 2007). Such 
adaptive foraging processes have often been neglected in ecological studies, although of potentially great importance for ecosystem functions such as predation and herbivory.

In agroecosystems, ants provide both ecosystem services and disservices as they facilitate piercing-sucking herbivores to obtain honeydew (cryptic herbivory), but also control major pests species such as Conopomorpha cramerella known to destroy up to $80 \%$ of cacao yields (Way and Heong 2009, Mollot et al. 2012, Offenberg et al. 2013, Gonthier et al. 2013, Wielgoss et al. 2014). Consequently, the balance of herbivory versus predation distinguishes biocontrol agents and pests. In general, tropical agroforestry provides high quality matrix habitats, but cacao agroforests with characteristic leaf flushing, flowering, and fruiting periods are highly dynamic (de Almeida and Valle 2007, Perfecto and Vandermeer 2008). In dynamic habitats, sap-feeding, honeydew-providing insects (e.g. aphids or mealybugs), and animal prey (e.g. caterpillar or beetles) underlie temporal boom and burst cycles related to host plant phenology. Mealybugs usually aggregate at fruits close to the fruit peduncle, that is why harvests change their abundances (personal observation, Ho and Khoo 1997). Till this day, it has rarely been investigated if spatiotemporal resource distribution alters species-specific food-mixing and, therefore, ecosystem service or disservice provision.

In a well-studied cacao agroforestry system in Central Sulawesi, Indonesia, we investigated such effects for a biocontrol agent, Dolichoderus thoracicus (SMITH, 1860), and an invasive pest species, Philidris cf. cordata (SMITH, 1859) (Ho 1994, Ho and Khoo 1997, Wielgoss et al. 2014). Both species hunt arthropods and collect honeydew of mealybugs such as Cataenococcus hispidus MORRISON (Hemiptera: Pseudococcidae) and other trophobionts (Ho and Khoo 1997, Wielgoss et al. 2014). We expect food mix, colony size, stoichiometric C:N balance, and foraging intensity of both species to be species-specific and to change with resource distribution. We tested the following hypotheses: (1) When animal prey is abundant, omnivorous ants incorporate additional prey in their food mix to increase colony growth, and thus $\delta^{15} \mathrm{~N}$ signatures, colony sizes, and foraging intensities increase, while C:N ratios drop. (2) Due to trophic plasticity, omnivorous ants compensate resource scarcity by adapting the food-mix (animal vs. herbal resource), hence maintaining stable colony sizes. (3) Biocontrol agents such as D. thoracicus incorporate more animal tissue in larval diets than pest species such as $P$. cf. cordata.

\section{Material and methods}

\section{Study sites and ant colony establishment.}

Between September 2011 and April 2012, we established 16 colonies of D. thoracicus within each of two plantations situated in the villages Wuasa (Napu valley, $1130 \mathrm{~m}$ a.s.l., 2000 - $3800 \mathrm{~mm}$ rainfall) and Sintuwu (Palolo valley, $620 \mathrm{~m}$ a.s.l., 2000 - $3000 \mathrm{~mm}$ rainfall) in Central Sulawesi, 
Indonesia. P. cf. cordata was absent in the Napu valley, most likely due to its preference for high temperatures (Wielgoss et al. 2010). We thus implemented 16 colonies in one agroforest of the Palolo valley. The agroforests were insecticide-free since March 2011 and contained some other trees (e.g. Erythrina subumbrans), intercrops (e.g. vanilla, manioc), and herbs (List S1). We introduced one ant colony to each of nine cacao trees in all four plots of each agroforest. One colony occupied four to six nesting tubes. We observed translocation of ant larvae between nesting tubes but not between trees. Cacao trees within one plot were three meters apart and the plots at least 10 meters. Branches were connected within a plot, but connections to the surrounding were pruned every 2 weeks. This ensured foraging was concentrated within the area we monitored. To obtain ant colonies, we added empty artificial nests to trees in different cacao plantations where the focal ant species were present (Fig.1). Fully occupied nests containing stable colonies (at least three months occupied, queens and brood present) were translocated into experimental plantations (Fig. 2, ant colony with cacao fruits and mealybugs). During the transport, all openings of the bamboo tube were closed with plastic sheets (not hermetic, but ant proof). The translocation was done within one hour. During the first two months, we supplemented with saturated sugar solution for carbohydrates and tinned tuna in salt water as protein source. Prior sampling, ant colonies were independent of additional food for at least three months. Thus, isotopic signatures of collected ant larvae were unaffected by supplementary feeding. Translocation and establishment processes were identical for both ant species.

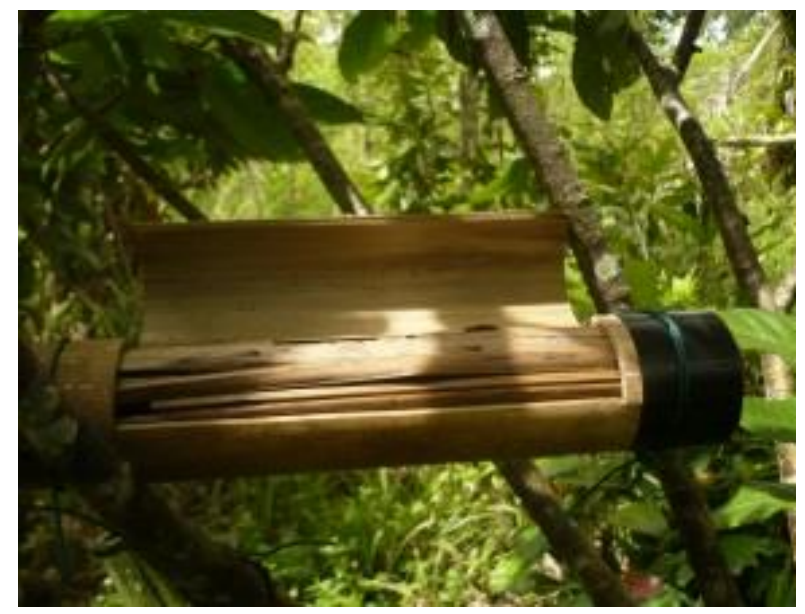

Fig. 1. Bamboo nest -introduced, not yet colonised

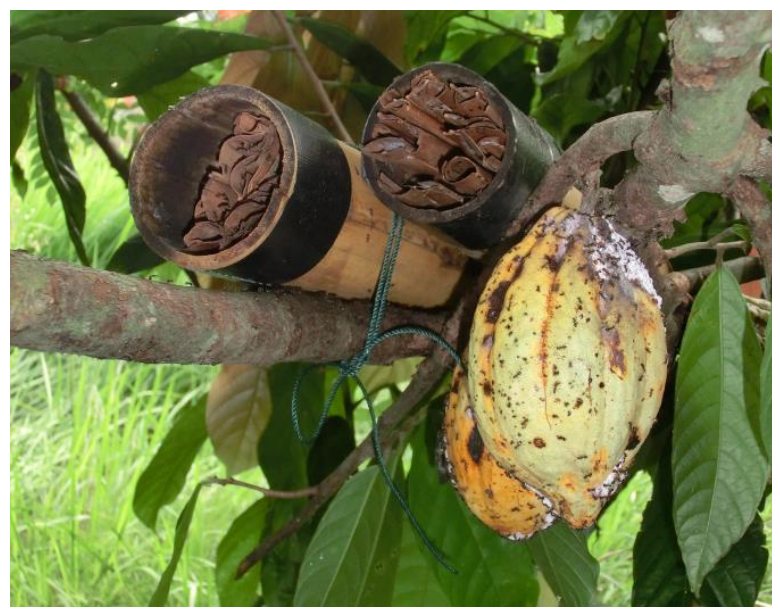

Fig. 2. Bamboo nests - colonised by D. thoracicus

\section{Sampling and survey methodology}

Field surveys: arthropods and plants. Within all three agroforests, we collected plant and arthropod samples, counted food sources, estimated the colony sizes, and conducted prey capture tests for each ant colony. At regular intervals, we monitored cacao tree phenology, ant colony size, and sampled ant larvae (younger three weeks), other arthropods, and plants for stable isotope analyses (Fig. 3). The assessment of cacao tree phenology started simultaneously with the 
ant colony establishment, and all nine trees of one plot were surveyed every two weeks. We counted flowers (open / closed) and fruits (Table S2; small, medium, big, ripe) and harvested ripe fruits. To avoid ant colony crashes due to trophobiont extraction, we sliced off pieces of fruit shell covered by trophobionts and placed it next to non-occupied fruits during harvest. We conducted four consecutive sampling sessions in the Napu valley and five in Palolo valley (Fig. 3). Each survey took one week. Prior to the first sampling, we randomly selected four ant colonies within each plot to be sampled consecutively every four weeks. If a colony disappeared (e.g. due to migration, or raids by other ants) we continued sampling another colony of the same plot instead. We estimated the abundance of ant workers, larvae, and queens while searching through the nest material to sample ant larvae tissue. During this process, we assigned each bamboo tube to one of the following categories: (1) few adults and few larvae, (2) moderate number of adults and few larvae, (3) many workers and moderate numbers of larvae, and (4) many workers and many larvae. Despite the disturbance, examined ant colony never abandoned the nesting tube. At the end of the experiment, we collected five nests of each category and plantation, kept it frozen for five days, and counted all individuals (see Table S3). We surveyed arthropods at four trees of each plot for 25 minutes. The abundances of Aphidoidea, Pseudococcidae and caterpillars were recorded by consecutively scanning the leafless trunks and branches $(5 \mathrm{~min}$ ) and tree crown (branches and leaves, totally 20 min) from each (five minutes) of four different, pairwise opposing directions. We measured the prey capture time using a maggot exposure experiment between two consecutive sampling dates (starting between second and third survey, Fig. 3).

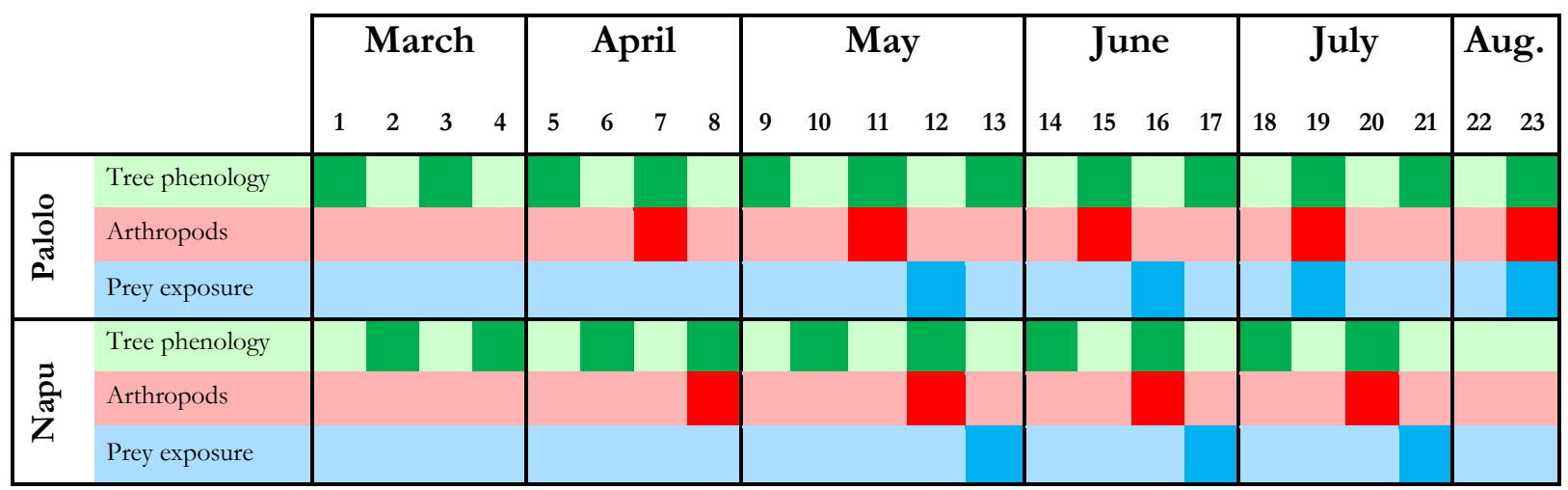

Fig. 3. Sampling scheme. Columns indicate the month and the experimental week (numbers) when the sampling was completed; rows indicate the valley: Napu valley $=1$ plantation, Palolo valley $=2$ plantations, and the type of survey: green $=$ cacao tree phenology, red $=$ arthropod sampling and counting (incl. ants), blue $=$ prey exposure experiment; dark coloured squares indicate the week in which the survey was done.

The maggots ( $\sim 4 \mathrm{~mm}$ length) were pinned at fruits of each tree consecutively three times. Freshly pinned maggots were observed till the first ant arrived or maximal 30 minutes. The observation was done by steadily walking between all exposure points. To standardize diurnal conditions, the 
experiment was conducted from 9:00 to 12:00 am. Maggots were reared open air using raw fish for approximately 5 days.

Stable isotope analyses: sampling tissues. We sampled three week-old ant larvae to link larval development and recent patterns of cacao phenology. While the life span of adult ants varies greatly, workers of $D$. thoracicus complete their larval development within 30 days under laboratory conditions (Indrarto 1993). In a pre-experiment, we showed a similar development pattern for $D$. thoracicus and P. cf. cordata within our study region (Description S4). Most collected ant larvae were younger than 3 weeks. We sampled locally abundant plants (T. cacao, shade trees, intercrops, and herbaceous plants, see List S1) by collecting $4 \mathrm{~cm}^{2}$ leaf tissues of two sun-growing and shadegrowing mature leaves, all originating from different shoots. Flower samples originated from eight different, randomly selected flower clusters (four closed and four opened flowers). We sampled cacao fruits by cutting out approximately $1 \mathrm{~cm}^{3}$ sized pieces at the fruit tip for four trees of each plot. If a tree did not harbor large, unripe fruits, we sampled small fruits instead. Arthropods were collected using canopy sweep netting and searching through the crown as well as on the stem of each tree. Within one plot, we searched for one hour. We took a subset (max 50\%) of aggregated individuals (Aphidoidea or Pseudococcidae). Samples of Conopomorpha cf. cramerella (SNELLEN, 1904) (Gracillariidae) - the cacao pod borer - were collected from harvested fruits, by storing all fruits of one plot in a sack and collecting hatching larvae over the course of three nights.

Stable isotope analyses: sample processing and analyses. Collected samples were placed in $2 \mathrm{ml}$ Eppendorf tubes and stored in a freezer $\left(-18^{\circ} \mathrm{C},<5 \mathrm{~h}\right.$ after sampling in the field). We transported frozen samples to Tadulako University, where we kept all samples frozen until drying in a ventilated drying cabinet. Dry samples were then stored in Eppendorf tubes, placed in plastic bags, and embedded in silica gel within hermetically sealed plastic boxes. In December 2012, the samples arrived at the Georg-August University Göttingen, were the silica gel (still dry in all cases) was replaced. All samples were kept in a dark, dry environment with a stable temperature of $16-18^{\circ} \mathrm{C}$. For stable isotope analyses, we used heads of individuals, except for ant larvae, trophobionts, and caterpillars which were analyzed complete bodied, since separating head and body was impossible. We milled leaf tissues using an electronic mill (Janke \& Kunkel, tabletop hammer mill type: MFC, $5000 \mathrm{r} / \mathrm{min}$ ), and pestled animal tissue. Depending on expected nitrogen contents, we measured 0.5 to $1 \mathrm{mg}$ animal tissue, and 1 - $2 \mathrm{mg}$ plant tissue using a micro scale (Sartorius micro, $0.001-3.000 \mathrm{mg}, \mathrm{d}<0.001 \mathrm{mg}$ ) to fill in tin capsules $(5 \times 8 \mathrm{~mm}$ ). Measurements of stable nitrogen isotope and C:N ratios were conducted by isotope mass spectrometry (Delta+, TermoFinnigan) coupled to an elemental analyzer (NA1110, CEE - Instruments). Stable isotope 
analyzes were conducted at the Centre for Stable Isotope Research and Analysis, Georg-August University Göttingen.

\section{Statistical analyses}

Data were analyzed using R 3.03 with the packages nlme 3.1.120, car 2.0.25, MASS 7.3.37, splines 3.0.2, and effects 3.0.3 (Wood 2000, Fox and Weisberg 2002, Venables and Ripley 2002, Fox 2003, Pinheiro and Bates 2014, R Core Team 2014).

Preparatory analyses. First, we modelled unstandardized $\delta^{15} \mathrm{~N}$ values of cacao to test the variation in the baseline isotope signal, using a linear mixed effects model. We included a compound-symmetry correlation structure to address temporal autocorrelation (model specification see below). To ensure comparability between different locations, we then standardized $\delta^{15} \mathrm{~N}$ values of arthropods by subtracting means of corresponding measurements of cacao leaves measured at the same plot.

Temporal patterns. We used linear mixed models (LME) and generalized linear mixed models fitted via PQL (glmmPQL) to model effects on the response variables: (1) ant larvae $\delta^{15} \mathrm{~N}$ signatures, (2) prey capture time, (3) C:N ratio, (4) colony size and all recorded, potential food sources namely: (5) cacao fruits, (6) cacao flowers, (7) young leaves, (8) caterpillars, and (9) trophobionts. To test the effects on the first four response variables, we used the following predictors: (1) ant species, (2) valley, (3) time, and (4) the interaction of time and species. We used normal distributed LME (responses 1-3) and Poisson distributed GLMMPQL-models (responses 4-9). We modeled temporal patterns by including the "time"-predictor (number of the consecutive survey 1-5) as B-spline basis matrix for a polynomial spline (bs function of the "splines" R package) and allowed 3 knots. The factors "ant species" and "valley" had two levels. The random effects included random slopes for "time", and ant colony nested within experimental plot nested within plantation (Plantations: D. thoracicus in Palolo, P. cf. cordata in Palolo, and D. thoracicus in Napu). If necessary, we used an exponential variance structure to meet assumptions of heteroscedasticity ("weights" function). To account for temporal autocorrelation we used an "autocorrelation-moving average correlation". In case of colony size we applied a compound symmetry correlation structure. We selected the best correlation structure by examining an AFC plot. Significance of predictors and interactions was assessed using likelihood-ratio tests using Type 2 sums of squares (R-package “car"). To test development of cacao fruits, cacao flowers, young cacao leaves, caterpillars, and trophobionts in the experimental plots, we used the same approach described above, but changed the predictors "ant species" and "valley" into the grouping variable "plantation". 
Correlations of habitat variables and ant colony responses. Finally, to test direct correlations of ant colony and habitat traits, we added plot-level estimators of cacao fruits, cacao flowers, young leaves, caterpillars, and trophobionts as linear predictors to final models of: (1) $\delta^{15} \mathrm{~N}$ signatures, (2) colony size, (3) C:N ratio, (4) prey capture time.

\section{Results}

Preparatory analysis. Prior to tackling the hypothesis, we tested the $\delta^{15} \mathrm{~N}$ baseline signatures (cacao leaves). Signatures varied between different plantations, but not over time (cacao leaves: $\left.\mathrm{F}_{\text {alley }}=11.21, \mathrm{P}<0.01, \mathrm{~F}_{\text {Time }}=1.11, \mathrm{P}=0.35\right)$. Hence, we applied small scale standardization of $\delta^{15} \mathrm{~N}$-meassurements by subtracting the local mean values of cacao leaves (Woodcock et al. 2012). Following, all mentioned isotopic signatures refer to standardized values.

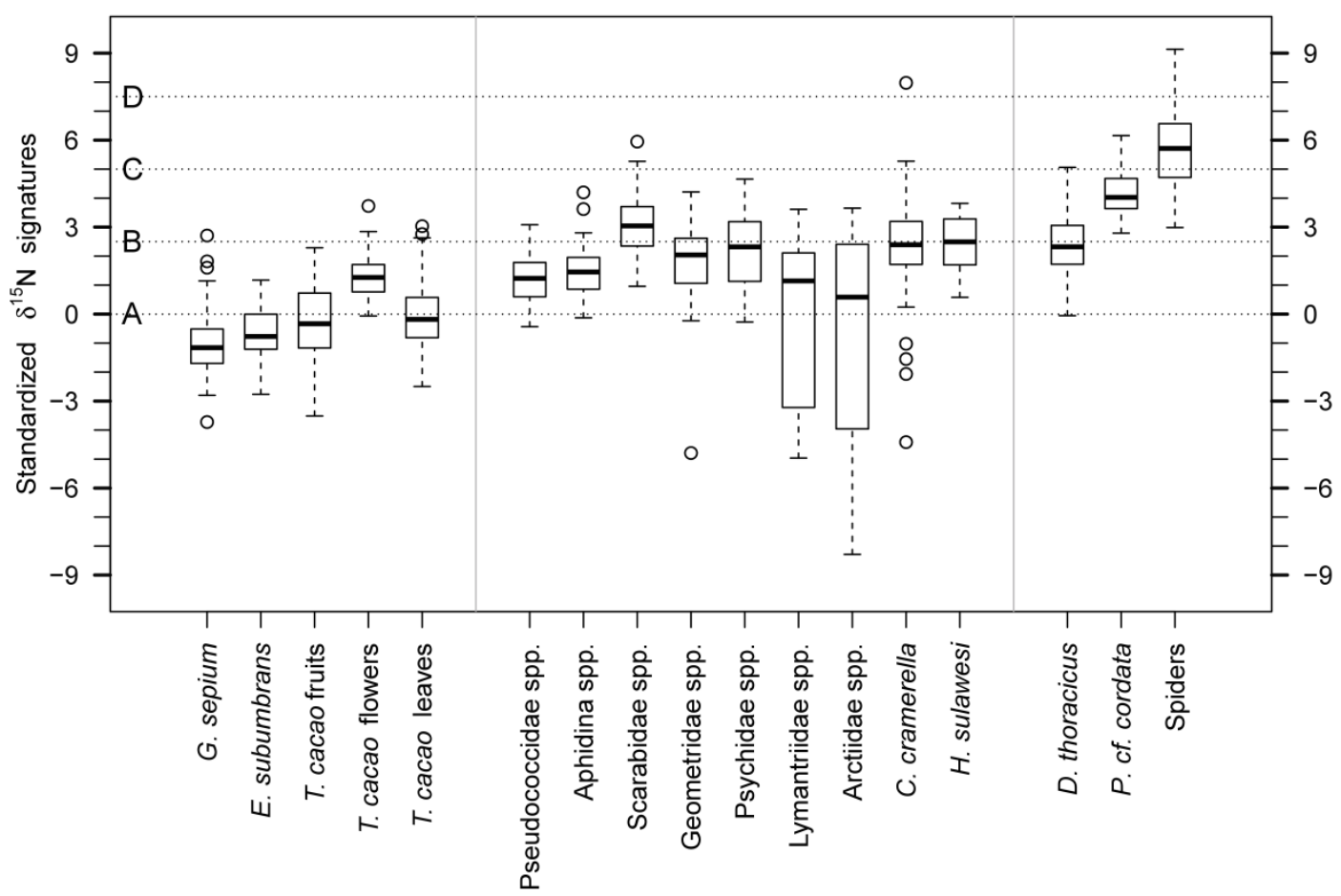

Fig. 4. Trophic positions of all recorded taxa in cacao plantations in Central Sulawesi, Indonesia. Boxes: bold lines are medians, hinges indicate first and second quartiles, whiskers extend to the most extreme data point which is no more than 1.5 times the length of the box away from the box, more extreme points are plotted as open circles. Dotted-horizontal lines indicate mean (range: \pm 1.25 - 1.25) value of one trophic level $\left(\mathrm{A}=\right.$ primary producer, $\mathrm{B}=1^{\mathrm{st}}$ order consumer (herbivores), $\mathrm{C}=2^{\text {nd }}$ order consumer (predators), $\mathrm{D}=3^{\text {rd }}$ order consumer (intra-guild predators).

Trophic positions. The overall trophic positions of all taxa were within the expected trophic range (Fig. 4). Leguminous shade trees Gliricidia sepium KUNTH and Erythrina subumbrans MERR. showed $\delta^{15} \mathrm{~N}$ values lower than cacao trees. Different cacao tree components varied in isotopic signatures, with flowers showing signatures $1.5 \delta^{15} \mathrm{~N}$ above leaves and fruits $\left(-0.5\right.$ to $\left.0 \delta^{15} \mathrm{~N}\right)$. Mean values of all plants were within the expected range of primary producers, ranging from -1.25 to $1.25 \delta^{15} \mathrm{~N}$. 
Mealybugs (Pseudococcidae spp.), aphids (Aphidina spp.), scarab beetles (Scarabidae spp.), spanworms (Geometridae spp), bagworms (Psychidae spp), tussock moths (Lymantriidae spp), and erebid moths (Arctiidae spp.) were classified as first order consumers (herbivores), showing $\delta^{15} \mathrm{~N}$ signatures between 1.25 and 3.75. Some samples of Lymantriidae and Arctiidae showed extreme isotope signatures. D. thoracicus larvae showed $\delta^{15} \mathrm{~N}$ signatures between 0 and 5.5, covering trophic positions of herbivores and predators. $P$. cf. cordata showed an average value of $4 \delta^{15} \mathrm{~N}$ with minima around 3.0 and maxima at 6.2. Hence, $P$. cf. cordata was classified as first and second order predator. Spiders occupied the top of the analyzed trophic web, and covered trophic positions of first, second, and third order predators $\left(3-9 \delta^{15} \mathrm{~N}\right)$.
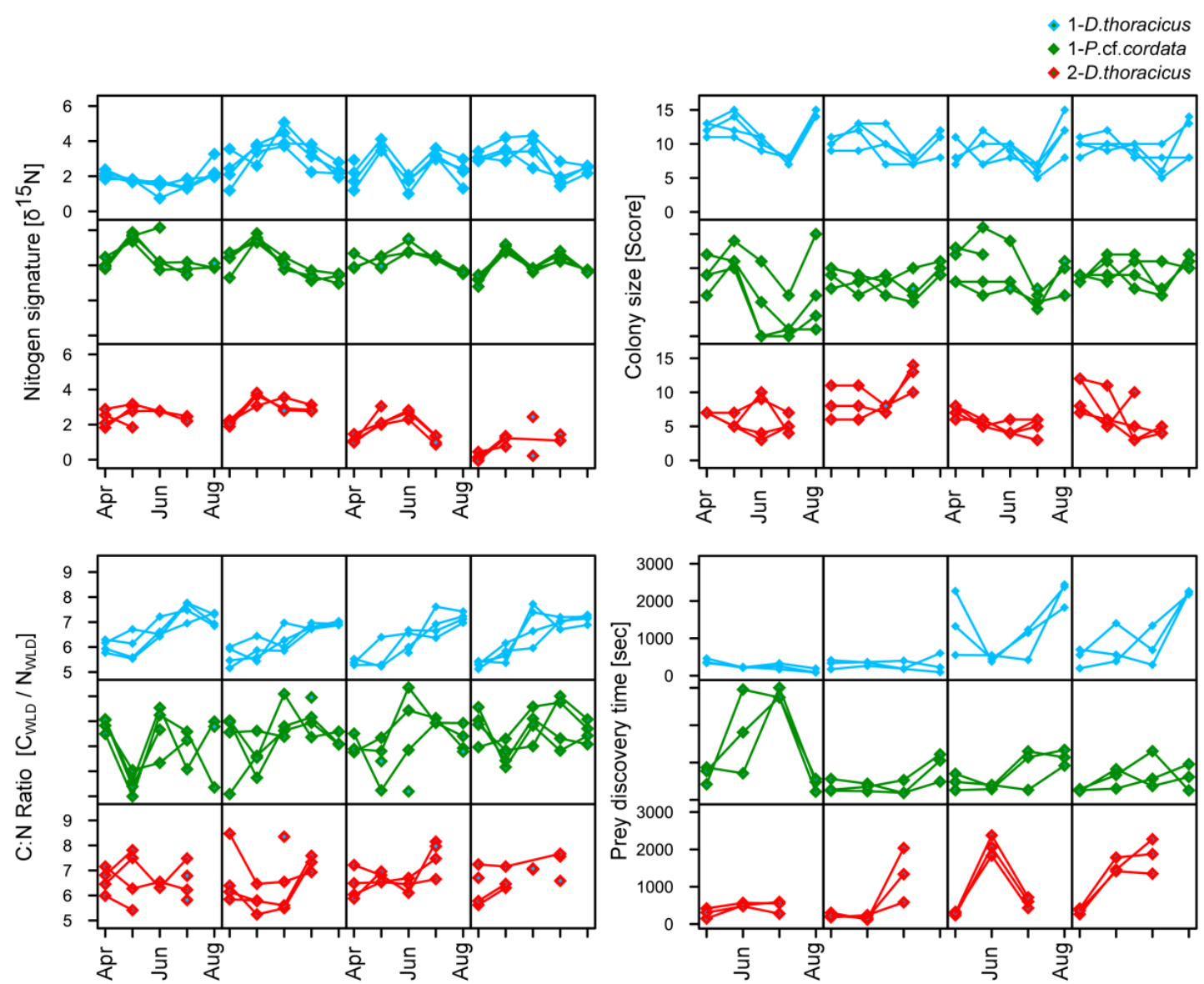

Fig.

5. Isotopic nitrogen signatures of ant colonies. (top-left), colony size (top-right), C:N ratio (bottom-left), and prey discovery time (bottom-right). X-axis: experimental month, Y-axis: response measurements as named, diamonds represent single colony values, lines connect colony specific values, different colors indicate different agroforests, numbers before ant species names indicate valleys containing the experimental agroforest $(1=$ Palolo, $2=\mathrm{Napu})$, figure sections with similar colored lines indicate 4 different plots of one agroforest.

Spatiotemporal and species-specific patterns of ant colonies. The $\delta^{15} \mathrm{~N}$ signatures of ant larvae were species-specific and reached colony-specific maxima between April and June (Fig 5, top-left). The signatures of most colonies dropped for approximately $2 \delta^{15} \mathrm{~N}$ till August. $P$. cf. 
cordata occupied trophic positions $2 \delta^{15} \mathrm{~N}$ higher than $D$. thoracicus. The temporal shift was similar in both species, and, for D. thoracicus, in both valleys. All colonies behaved similar within, but slightly different between plots of one plantation. ( $\mathrm{F}$ species $=9.94, \mathrm{Df}=1, \mathrm{P}<0.01 ; \mathrm{F}$ Time $=71.28$, $\mathrm{Df}=3, \mathrm{P}<0.01$; Chi species:Time $=3.73, \mathrm{Df}=3, \mathrm{P}=0.29$; Chi valley $=0.67, \mathrm{Df}=1, \mathrm{P}=0.42$; “:” indicates interactions; " $\mathrm{n}$ " = ungrouped number of observations). The colony size tended to be species-specific and decreased non-linear for approximately 2 units over time (Fig 5, top-right). The reduction tended to be species-specific, with $P$. cf. cordata being more resilient and the colonies being larger for $D$. thoracicus in the Palolo valley (Chi ${ }_{\text {species }}=2.96, \mathrm{Df}=1, \mathrm{P}=0.09$; $\mathrm{Chi}_{\text {Time }}=70.06$, $\mathrm{Df}=3, \mathrm{P}<0.01$; Chi species:Time $=6.85, \mathrm{Df}=3, \mathrm{P}=0.08$; Chi valley $=22.99, \mathrm{Df}=1, \mathrm{P}<0.01)$.
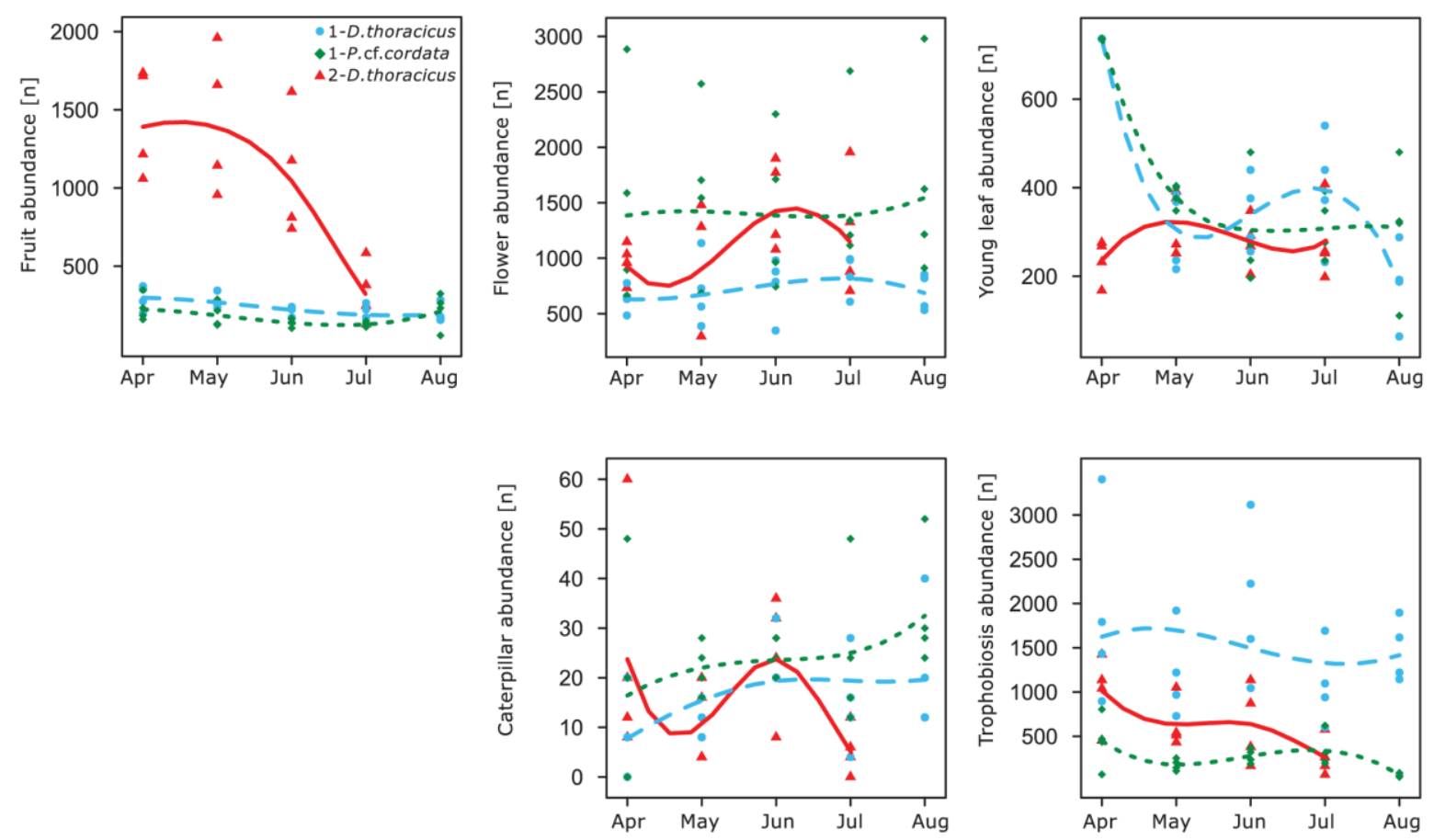

Fig. 6. Temporal patterns of resource availability. $\mathrm{x}$ axis: experimental month, $\mathrm{y}$ axis: counted abundance of cacao fruits, cacao flowers, young cacao leaves, caterpillars and trophobionts. Lines show model predictions, diamonds show counted values of one plot, different colors indicate different agroforests, numbers before ant species names indicate valleys containing the experimental agroforest $(1=$ Palolo, $2=\mathrm{Napu})$.

The C:N ratio of $P$. cf. cordata colonies showed a high deviation (span: 5-8) between different colonies, and moderately increased over the course of the experiment (Fig 5, bottom-left). While $D$. thoracicus colonies showed patterns similar to $P$. cf. cordata in the Napu valley, C:N ratios of $D$. thoracicus colonies in Palolo linearly increased from five to seven units between April and August $\left(\right.$ Chi $_{\text {species }}=37.25, \mathrm{Df}=1, \mathrm{P}<0.01 ; \mathrm{Chi}_{\text {Time }}=33.58, \mathrm{Df}=3, \mathrm{P}<0.01$, Chi species:Time $=18.95, \mathrm{Df}$ $=3, \mathrm{P}<0.01$, Chi valley $=22.99, \mathrm{Df}=1, \mathrm{P}<0.01)$. The prey discovery time increased for both species and locations during the experiment (Fig 5, bottom-right). P. cf. cordata discovered prey faster than D. thoracicus. Colonies of D. thoracicus were the slowest in Napu (Chi species $=4.42, \mathrm{Df}=1$, 
$\mathrm{P}=0.03$; Chi $_{\text {Valley }}=15.14, \mathrm{Df}=1, \mathrm{P}<0.01$ Chi $_{\text {Time }}=11.48, \mathrm{Df}=1, \mathrm{P}<0.01$, Chi species:Time $=$ 0.002 , Df $=1, \mathrm{P}=0.97)$. The colony size and $\delta^{15} \mathrm{~N}$ signatures of ant larvae were independent of each other $(\mathrm{Chi}$ ant abundance $=0.17, \mathrm{Df}=1, \mathrm{P}=0.68)$.

Table 1 Correlation of habitat phenology and ant colony response (ANOVA Type II).

\begin{tabular}{|c|c|c|c|c|c|}
\hline Response & Added Predictor & Chisq & Df1, Df2 & $\operatorname{Pr}(>$ Chisq $)$ & Effect direction \\
\hline \multicolumn{6}{|c|}{ S15N signature } \\
\hline & Young leaves & 0.68 & 1,150 & 0.41 & \\
\hline & Flowers & 0.82 & 1,150 & 0.36 & \\
\hline & Fruits & 2.12 & 1,150 & 0.15 & \\
\hline & Caterpillars & 3.52 & 1,150 & 0.06 & positive \\
\hline & Trophobionts & 0.52 & 1,150 & 0.47 & \\
\hline \multicolumn{6}{|l|}{ Colony size } \\
\hline & Young leaves & 4.95 & 1,160 & 0.03 & negative \\
\hline & Flowers & 0.16 & 1,160 & 0.69 & \\
\hline & Fruits & 3.20 & 1,160 & 0.07 & negative \\
\hline & Caterpillars & 6.91 & 1,160 & $<0.01$ & positive \\
\hline & Trophobionts & 5.08 & 1,160 & 0.02 & positive \\
\hline \multicolumn{6}{|l|}{ C:N Ratio } \\
\hline & Young leaves & 0,11 & 1,150 & 0.75 & \\
\hline & Flowers & 1.35 & 1,150 & 0.25 & \\
\hline & Fruits & 0.01 & 1,150 & 0.91 & \\
\hline & Caterpillars & 0.03 & 1,150 & 0.86 & \\
\hline & Trophobionts & 1.45 & 1,150 & 0.23 & \\
\hline \multicolumn{6}{|c|}{ Prey discovery time } \\
\hline & Young leaves & 0.35 & 1,93 & 0.55 & \\
\hline & Flowers & 4.03 & 1,93 & 0.045 & positive \\
\hline & Fruits & 11.42 & 1,93 & $<0.01$ & Negative \\
\hline & Caterpillars & 0.02 & 1,93 & 0.88 & \\
\hline & Trophobionts & 0.81 & 1,93 & 0.37 & \\
\hline
\end{tabular}

Spatiotemporal resource distribution. During the experiment, cacao tree phenology and food availability significantly changed (Fig. 6). The number of recorded cacao fruits moderately decreased from $\sim 350$ to $\sim 250$ fruits in both plantations of the Palolo valley. In the D. thoracicus plantation of the Napu valley, fruit abundances dropped suddenly from $\sim 1500$ to $\sim 500$. The P. cf. cordata plantation in Palolo contained the fewest fruits $($ Chi Plantation $=162.2, \mathrm{Df}=2, \mathrm{P}<0.01$; Chi $_{\text {Time }}=91.73, \mathrm{Df}=3, \mathrm{P}<0.01 ;$ Chi Plantation:Time $\left.=74.95, \mathrm{Df}=6, \mathrm{P}<0.01\right)$. Flower abundances moderately increased in both plantations of the Palolo valley, but steeply increased from 1000 to 1500 flowers in the Napu valley. In Palolo, plots of P. cf. cordata ( 1500 flowers) contained 3 times more flowers than D. thoracicus plots (Chi Plantation $=7.33, \mathrm{Df}=2, \mathrm{P}=0.06$ Chi $_{\text {Time }}=9.13, \mathrm{Df}=3$, $\mathrm{P}=0.01$; Chi Plantation:Time $=15.74, \mathrm{Df}=6, \mathrm{P}=0.02)$. Abundances of young leaves fluctuated nonlinear, moderately increasing in the Napu valley (220 to 300 leaves), but steeply decreasing ( 700 to 300 leaves) in Palolo (Chi Plantation $=26.89, \mathrm{Df}=2, \mathrm{P}<0.01$; Chi ${ }_{\text {Time }}$ 114.97, $\mathrm{Df}=3, \mathrm{P}<0.01$; 
Chi Plantation:Time $=44.90, \mathrm{Df}=6, \mathrm{P}<0.01)$. Numbers of caterpillars doubled in both plantations of the Palolo valley from 10 to 20 individuals. Caterpillar abundances in D. thoracicus plots of the Napu valley showed a maximum between April and June, but, overall, decreased from over 40 to 5 individuals $\left(\right.$ Chi Plantation $=1.98, \mathrm{Df}=2, \mathrm{P}=0.37$; Chi ${ }_{\text {Time }} 4.07, \mathrm{Df}=3, \mathrm{P}=0.25$; Chi Plantation:Time $=18.45, \mathrm{Df}=6, \mathrm{P}<0.01)$. Amounts of trophobionts moderately decreased $(\sim 5 \%)$ in both Palolo valley plantations, but steeply dropped from 1000 to 300 individuals in the Napu valley $D$. thoracicus plantation $\left(\right.$ Chi Plantation $=51.96, \mathrm{Df}=2, \mathrm{P}<0.1$; Chi Time $_{\text {7.15, }} \mathrm{Df}=3, \mathrm{P}=0.07$; Chi Plantation:Time $=17.58, \mathrm{Df}=6, \mathrm{P}<0.01)$.

Correlation of habitat variables and ant colony responses. Finally, we correlated habitat phenology and food resource abundance with all four ant colony responses: (1) isotopic nitrogen signature, (2) colony size, (3) C:N ratio, and (4) prey discovery time (Table 1). The $\delta^{15} \mathrm{~N}$ signature of ant larvae tended to positively correlate with amount of available prey (caterpillar abundance). The ant colony size was negatively correlated with numbers of young leaves and fruits, but positively with abundances of caterpillars and trophobionts. The C:N ratio was not influenced by any of the predictors. Both species needed more time to discover prey when many flowers were present, but less when many fruits were available.

\section{Discussion}

Trophic positions. D. thoracicus and $P$. cf. cordata showed high trophic plasticity, although P. cordata acted as intra-guild predator showing $\delta^{15} \mathrm{~N}$ signatures similar to spiders. When fruits became scarce, both species used more plant-derived resources, colony sizes decreased, and C:N ratios as well as prey discovery time increased. These patterns were most likely caused by differences in availability of potential prey and mealybugs, which are tended by ants to produce honeydew. Plant phenology had an impact on the detection time of exposed prey, which confirms our expectation, namely that shifts in tree phenology and associated changes in food sources affect the trophic function and predatory effectiveness of ants.

As expected, both ant species occupied different trophic levels, but responded similarly to the spatiotemporally changing environment. We assumed a $2.5 \delta^{15} \mathrm{~N}$ inter-level distance for distinct trophic positions of primary producers, herbivores, and predator. Such an inter-level distance is comparable to other studies presenting different consumers separated by $2.3-3.3 \delta^{15} \mathrm{~N}$ (Davidson et al. 2003, Blüthgen et al. 2003, Hood-Nowotny and Knols 2007, International Atomic Energy Agency 2009). The trophic separation was clear, hence, we assigned all taxa to expected guilds with producers (e.g. cacao, legume shade trees) at the bottom, herbivores (e.g. mealybugs, aphids, and caterpillars) at medium levels, and first as well as second order predators (e.g. ants and spiders) at 
the top. The $\delta^{15} \mathrm{~N}$ signatures of the plant tissue are typical for legume dominated habitats, and previous studies found similar signatures for cacao and shade trees (Anhar 2005). Lymantriidae and Arctiidae had low $\delta^{15} \mathrm{~N}$ signatures, as these orders contain species feeding on lichens (e.g. Lithosiinae among the Arctiidae) with naturally very low $\delta^{15} \mathrm{~N}$ signatures. Increased isotopic signatures of Scarabidae could have been caused by feeding on actively growing, young leaf tissue (e.g. flowers, young leaves, and small fruits). Such tissue shows naturally high $\delta^{15} \mathrm{~N}$ signatures (Anhar 2005). Outlying values of $C$. cramerella can be due to recycling resources during metamorphose (Doi et al. 2007). Variation in $\delta^{15} \mathrm{~N}$ signatures of mealybugs and aphids can be caused by consumption of saps of different plant parts in different growth periods (e.g. cacao flowers, fruits, leaves). We are confident that measured $\delta^{15} \mathrm{~N}$ signatures reflect the adequate trophic positions, as we corrected for the isotopic habitat baseline signal using cacao leaves, the most abundant and continuously distributed food resource (Woodcock et al. 2012, Pfeiffer et al. 2013). Isotopic signatures of analyzed ant larvae represent diets obtained before sampling, as isotopic signatures of whole bodied samples are determined by most recently digested foods (Blüthgen et al. 2003, Tillberg et al. 2006). Consequently, presented isotopic values should fit to counted resource abundances.

Environmental influence on ants. Here, intraspecific shifts of trophic positions were mediated by availability of prey. More animal prey led to increasing trophic positions of both species. An expected mechanism, as foraging behavior of ants depend on biotic and abiotic seasonality of the habitat and omnivores use most abundant resources (Eubanks and Denno 1999, Rudolph and Palmer 2013, Warren and Chick 2013). In our study, increasing prey availability was correlated with increasing colony sizes, a pattern underlining the capability of omnivores to incorporate additional prey to facilitate colony growth. On the other hand, decreasing numbers of trophobionts lowered colony sizes, but did not alter trophic positions. Consequently, tested ants do not compensate carbohydrate scarcity by increasing prey foraging to maintain stable numbers of brood (but see Rudolph and Palmer 2013). Ant workers demand regular carbohydrate intake to maintain their metabolism (Haack et al. 1995, Blüthgen et al. 2003). A recent study has shown that worker mortality increases dramatically when ants are fed a protein-rich diet, with strong effects on colony size within a few days from the diet shift (Dussutour and Simpson 2012). Consequently, ant worker survivability (i.e. colony size) is closely related to the obtained honeydew.

In our study, trophic positions and colony sizes of the ants decreased, while the stoichiometric balance of larval diets shifted towards higher proportions of carbohydrate. We analyzed whole bodied ant larvae, hence we obtained stoichiometric signatures partly influenced by recently fed, undigested food (Tillberg et al. 2006). Usually, animal prey is limited and scattered 
distributed, that is why numerical abundant arboreal ants depend on herbaceous diets to maintain worker biomass (Tobin 1991, Davidson et al. 2003). A decline of trophobiosis partner can induce ants to focus plant-derived, easy defendable and stationary resources such as mealybug aggregations (Davidson 1998). Consequently, the proportion of nitrogen derived of resources with high C:N ratios, such as plants or arthropods of lower trophic levels increases. Simultaneously, density adaptation can take place, as we observed $D$. thoracicus and $P$. cf. cordata killing nest-mates and producing smaller bodied workers when starving (personal observation). Small individuals with flimsy exoskeletons need little nitrogen during larval growth, and are typical for arboreal ants living of nitrogen poor resources (Davidson and Patrell-Kim 1996, Cook and Davidson 2006). Such a speculative resource-dependent density adaptation process could explain the shown positive correlations of colony size with caterpillar or trophobiont abundance. Shown negative correlations of ant colony size and fruit or young leave abundances support the theory described above.

Ants and predators, such as spiders, live antagonistic. Thereby, ants serve as prey of jumping and web spiders, but prey on spiders as well (Sanders and Platner 2007, Pathummal Beevi and Mahapatro 2008). Abundances of fruits and young leave indicate predator hiding opportunities due to microhabitat structures. Especially, spider webs live close to stems of young leaves, shoots, buds, fruiting and flowering pedicels - the preferred, nitrogen-rich feeding sites of aphids and mealybugs (Nixon 1951, Wielgoss et al. 2014, personal observation). Increasing abundances of fruits and leaves could have provided additional cover for ambush predators such as predatory bugs, spiders, and earwigs, but not necessarily provided additional feeding sites for ant trophobionts. Under high predation pressure, ants focus foraging on easy accessible and defendable resources to lower investment in resource discovery (Davidson 1998). Increasing predation risk explains decreasing ant colony sizes and simultaneously increasing prey discovery time. A trade-off between resource discovery and resource defense can be species as well as habitat specific (Wilson 1971).

The temporal patterns of trophic plasticity reported here emphasize the ants' trophic variability (predation vs. cryptic herbivory) in agricultural habitats due to resource shortages. Such resource boom-and-burst cycles are typical in cacao agroforestry and further enhanced by seasonal harvesting (de Almeida and Valle 2007). Abundances of mealybugs and aphis fluctuate due to resource distribution, reproduction patterns, and predator presence (Caasi-Lit et al. 2012). Trophic plasticity among ant colonies of a single species can cover $6 \delta^{15} \mathrm{~N}$, in other words two trophic levels (DeNiro and Epstein 1981, Cabana and Rasmussen 1994, Mooney and Tillberg 2005, Tillberg et al. 2007). Consequently, a combination of trophic plasticity and density adaptation are most likely the reasons for the ants' ability to live in and adapt to highly dynamic, anthropogenic shaped 
ecosystems. Thereby, particular ants can switch between animal-derived and plant-derived resources, impacting species-specific ecosystem service provision.

Nutritional strategies of native and invasive ants in agroforestry. $D$. thoracicus and $P$. cordata belong to the Dolichoderinae subfamily, which is one of the most exudate-depending subfamilies of ants (Cook and Davidson 2006). Moreover, the Dolichoderinae represent a phylogenetic unclear species complex, including a group of species classified as Dolichoderus thoracicus being native to Sulawesi, Indonesia (Maschwitz et al. 1991, Shattuck 1994). The Genus Philidris is distributed from eastern India to northern Australia including Indonesia (Shattuck 1992). The native distribution of Philidris cordata is unknown, but most samples are known from Papua and Northern-Australia ("AntWeb” 2015). In Sulawesi, P. cordata colonized the Palolo valley during the last decades and is absent in the remote located Napu valley (pers. communication Arno Wielgoss, unpublished data PG).

Both species colonize the canopy of cacao plantations and depend on trophobiosis (Wielgoss et al. 2014). High C:N ratios and strong chemical weaponry of $P$. cordata indicates a strong demand of trophobiosis partners to obtain honeydew (Davidson and Patrell-Kim 1996, Davidson 1998). High proportions of liquid food are common in tropical canopy ants and European Formicinae, both deriving large fractions of nitrogen from nectar or honeydew, but the importance of trophobionts is species-specific (Floren et al. 2002, Fiedler et al. 2007, Seifert 2007). The relatively low nitrogen content of honeydew can be equalized by relatively high amounts of individual honeydew consumption, due to long life-spans (Davidson and Patrell-Kim 1996). The digestive modifications (Eisner 1957) to efficiently process large quantities of liquid food with low nitrogen contents is absent in the genus Dolichoderus, but present in other Dolichoderinae (Davidson and Patrell-Kim 1996). Increased honeydew intake might explain why P. cordata showed much higher C:N ratios in larval tissues compared with $D$. thoracicus. Such, high C:N ratios are common for ants using nitrogen-free chemical weaponry (Davidson and Patrell-Kim 1996), being highly territorial (Vepsäläinen and Pisarski 1982), and conduct high tempo activities such as dynamic worker allocation or rapid resource discovery (Oster and Wilson 1978). Such a behavior is common for P. cf. cordata (personal observation).

Honeydew consumption might influence the isotopic nitrogen signature, as nitrogen upgrading bacteria live in the guts of ants favoring plant-derived resources such as many Formicinae and Dolichoderinae (Cook and Davidson 2006). These bacteria could discriminate nitrogen in favor of $\delta^{15} \mathrm{~N}$. If so, ants using honeydew as primary resource, show very low $\delta^{15} \mathrm{~N}$ signatures (Davidson and Patrell-Kim 1996). Associated endosymbiotic bacteria are suspected to lower isotopic signatures, making the analyzed organisms theoretically more herbivorous (Cook and Davidson 2006). These endosymbiotic bacteria do upgrade nitrogen to provide essential amino 
acids (Feldhaar et al. 2007). For D. thoracicus and P. cordata it is still unknown whether or not they harbor endosymbiotic bacteria. Nevertheless, many Dolichoderinae (including $D$. thoracicus and $P$. cordata) store fed honeydew in their abdomen where endosymbiotic gut bacteria could synthesize essential amino acids, available for nets mates through anal trophallaxis (Cook and Davidson 2006). An explanation for increased $\delta^{15} \mathrm{~N}$ signatures could be ants feeding on fungi, which have higher nitrogen signatures than plants of the habitat (Gebauer and Taylor 1999), but neither D. thoracicus nor P. cordata are known to be fungivory. Other supplementary nitrogen sources of arboreal ants are bird feces (Roche and Wheeler 1997). We did not observe ants feeding feces, but the consumption could alter isotopic signatures in any direction, due to bird diets. Accordingly, ant diets are influenced by evolutionary, species-specific adaptations to habitats providing specific sets of usable resources.

How to distinguish ecosystem service and disservice providers? The "resource balance model" related to "foraging tempo theory", as discussed in Davidson (1997), may serve as valid explanation of species-specific nutritional needs resulting in ecosystem service or disservice provision. Ants of the subfamily Dolichoderus are usually "low-tempo foragers" - some species might be "high-tempo foragers" - and, therefore, require different stoichiometric balances of carbon and nitrogen derived of food resources (Hölldobler and Wilson 1990, Davidson 1997). Species-specific variation of foraging patterns due to food item stoichiometry finds support in our study, as $P$. cf. cordata showed a higher C:N ratio, discovered prey faster, and showed higher $\delta^{15} \mathrm{~N}$ signatures than $D$ thoracicus. We infer, that $D$. thoracicus relies on honeydew to fuel workers and preys on herbivores to feed the larvae, while P. cf. cordata uses honeydew to fuel workers and to derive nitrogen for larval diets. Additionally, P. cf. cordata supplements larval diets with animal tissue of high trophic levels due to intra-guild predation. The morphological incapability of D. thoracicus to derive sufficient amounts of nitrogen from honeydew, due to stomach morphology, as discussed above, might be essential for conducted biocontrol. Such a behavioral response and species-specific diets may help to explain different impacts on yield, herbivorous pests of cacao reported previously (Wielgoss et al. 2014). We conclude that trophobionts and arthropod prey are necessary to maintain stable colony size, but access to arthropod prey is mandatory to avoid crypticherbivory due to focusing trophobionts when resources are generally scarce.

\section{Acknowledgements}

We are grateful to our Indonesian collaborates at Tadulako University, Agricultural University Bogor (IPB), and all administrative bodies. We thank A. Rizali for ant identification, and H. Mangopo (trees) as well as Firdaus (herbs) for plant identification. A. Wielgoss and I. Motzke 
we thank for support and guidance during field work. We thank J. Dyckmans (KOSI, Göttingen) for advices regarding isotope analyses and N. Holm for sample preparation. For the great encouragement we thank all assistants Ivon, Abe, Fatma, Nimus, Idi, Soni, and Sardin. We appreciate the work of the German coordination teams of the DFG projects ELUC (CL-474/1-1) and Collaborative research center 990 (EFForTS), especially W. Lorenz. Finally, we acknowledge the DFG founding of the projects ELUC (CL-474/1-1) and collaborative research center 990 (EFForTS).

\section{References}

Anhar, A. 2005. The role of biological nitrogen fixation in the cacao agroforestry system in Central Sulawesi Indonesia. Dissertation. Göttingen.

AntWeb. 2015. https://www.antweb.org/description.do?genus=philidris\&species=cordata\& rank $=$ species\&project $=$ atolants.

Blüthgen, N., G. Gebauer, and K. Fiedler. 2003. Disentangling a rainforest food web using stable isotopes: dietary diversity in a species-rich ant community. Oecologia 137:426-435.

Caasi-Lit, M. T., I. L. Lit Jr., and A. R. Larona. 2012. Expansion of Local Geographic and Host Ranges of Nipaecoccus nipae ( Maskell) (Pseudococcidae, Hemiptera) in the Philippines with New Records of Predators and Attending Ants. Philippine Journal of Crop Science $37: 47-56$.

Cabana, G., and J. Rasmussen. 1994. Modelling food chain structure and contaminant bioaccumulation using stable nitrogen isotopes. Nature 372:255-257.

Cook, S. C., and D. W. Davidson. 2006. Nutritional and functional biology of exudate-feeding ants. Entomologia Experimentalis et Applicata 118:1-10.

Davidson, D. W. 1997. The role of resource imbalances in the evolutionary ecology of tropical arboreal ants. Biological Journal of the Linnean Society 61:153-181.

Davidson, D. W. 1998. Resource discovery versus resource domination in ants: a functional mechanism for breaking the trade-off. Ecological Entomology 23:484-490.

Davidson, D. W., S. C. Cook, R. R. Snelling, and T. H. Chua. 2003. Explaining the Abundance of Ants in Lowland Tropical Rainforest Canopies. Science 300:969-972.

Davidson, D. W., and L. Patrell-Kim. 1996. Chapter 7 Tropical Arboreal Ants : Why So Abundant? Pages 127-140 in A. C. Gibson, editor. Neotropical Biodiversity and Conservation,. University of California, Los Angeles.

de Almeida, A.-A. F., and R. R. Valle. 2007. Ecophysiology of the cacao tree. Brazilian Journal Of Plant Physiology 19:425-448. 
DeNiro, M. J., and S. Epstein. 1981. Influence of diet on the distribution of nitrogen isotopes in animals. Geochimica et Cosmochimica Acta 45:341-351.

Doi, H., E. Kikuchi, S. Takagi, and S. Shikano. 2007. Changes in carbon and nitrogen stable isotopes of chironomid larvae during growth, starvation and metamorphosis. Rapid Communications in Mass Spectrometry 21:997-1002.

Dussutour, A., and S. J. Simpson. 2012. Ant workers die young and colonies collapse when fed a high-protein diet. Proceedings of the Royal Society B: Biological Sciences 279:2402-2408.

Eisner, T. 1957. A comparative morphological study of the proventriculus of ants (Hymenoptera: Formicidae). Bulletin of The Museum of Comparative Zoology 116:437-490.

Eubanks, M. D., and R. F. Denno. 1999. The ecological consequences of variation in plants and prey for an omnivorous insect. Ecology 80:1253-1266.

Feldhaar, H., J. Straka, M. Krischke, K. Berthold, S. Stoll, M. J. Mueller, and R. Gross. 2007. Nutritional upgrading for omnivorous carpenter ants by the endosymbiont Blochmannia. BMC biology 5:48.

Fiedler, K., F. Kuhlmann, F. M. Steiner, G. Gebauer, B. C. Schlick-Steiner, F. M. Steiner, and G. Gebauer. 2007. Stable N-isotope signatures of central European ants - Assessing positions in a trophic gradient. Insectes Sociaux 54:393-402.

Floren, A., A. Biun, and E. Linsenmair. 2002. Arboreal ants as key predators in tropical lowland rainforest trees. Oecologia 131:137-144.

Folgarait, P. J. 1998. Ant biodiversity and its relationship to ecosystem functioning: a review. Biodiversity and Conservation 7:1221-1244.

Fox, J. 2003. Effect Displays in $\{R\}$ for Generalised Linear Models. Journal of Statistical Software 8:1-27.

Fox, J., and S. Weisberg. 2002. An \{R\} Companion to Applied Regression. Sage Publications.

Gebauer, G., and a. F. S. Taylor. 1999. $\delta 15 \mathrm{~N}$ natural abundance in fruit bodies of different functional groups of fungi in relation to substrate utilization. New Phytologist 142:93-101.

Gonthier, D. J., K. K. Ennis, S. M. Philpott, J. Vandermeer, and I. Perfecto. 2013. Ants defend coffee from berry borer colonization. BioControl 58:815-820.

Grover, C. D., A. D. Kay, J. A. Monson, T. C. Marsh, and D. A. Holway. 2007. Linking nutrition and behavioural dominance: carbohydrate scarcity limits aggression and activity in Argentine ants. Proceedings. Biological sciences / The Royal Society 274:2951-2957.

Haack, K. D., S. B. Vinson, and J. K. Olson. 1995. Food distribution and storage in colonies of Monomorium pharaonis (L.) (Hymenoptera: Formicidae). J. Entomol. Sci. 30:70-81.

Ho, C. 1994. Methods Towards Efficient Establishment of Introduced Black cocoa Ant , 
Dolichoderus thoracicus for Natural Control of Helopelfis theivora Damage in Cocoa. The planter 70:487-495.

Ho, C., and K. Khoo. 1997. Partners in biological control of cocoa pests: mutualism between Dolichoderus thoracicus (Hymenoptera: Formicidae) and Cataenococcus hispidus (Hemiptera: Pseudococcidae). Bulletin of Entomological Research 87:461-470.

Hölldobler, B., and E. O. Wilson. 1990. The Ants. Belknap Press of Harvard University Press, Massachusetts.

Hood-Nowotny, R., and B. G. J. Knols. 2007. Stable isotope methods in biological and ecological studies of arthropods. Entomologia Experimentalis et Applicata 124:3-16.

Indrarto, I. 1993. Life history of the black ant (Dolichoderus thoracicus Smith) in association with the mealybug (Planococcus lilacinus (Ckll.)) and its effects on regulating Helopeltis bakeri Poppius population. University of the Philippines at Los Baños.

International Atomic Energy Agency. 2009. Manual for the Use of Stable Isotopes in Entomology. Vienna.

Maschwitz, U., K. Dumpert, T. Botz, and W. Rohe. 1991. A silk-nest weaving Dolichoderine ant in a Malayan rain forest. Insectes Sociaux 38:307-316.

Mollot, G., P. Tixier, F. Lescourret, S. Quilici, and P.-F. Duyck. 2012. New primary resource increases predation on a pest in a banana agroecosystem. Agricultural and Forest Entomology $14: 317-323$.

Mooney, K. A., and C. V. Tillberg. 2005. Temporal and spatial variation to ant omnivory in pine forests. Ecology 86:1225-1235.

Nixon, G. E. J. 1951. The Association of Ants with Aphids and Coccids. Commonwealth Institute of Entomology, London.

Offenberg, J., N. Thi Thu Cuc, and D. Wiwatwitaya. 2013. The effectiveness of weaver ant ( Oecophylla smaragdina) biocontrol in Southeast Asian citrus and mango. ASIAN MYRMECOLOGY 5:139-149.

Oster, G. F., and E. O. Wilson. 1978. Caste and ecology in the social insects. Monographs in population biology 12 .

Pathummal Beevi, S., and G. K. Mahapatro. 2008. Species-spectrum and inter-relationship between ant and spider fauna in cashew agro- ecosystem Species-spectrum and inter-relationship between ant and spider fauna in cashew agro-ecosystem. Journal of Plantation Crops 36:0000 .

Perfecto, I., and J. Vandermeer. 2008. Biodiversity Conservation in Tropical Agroecosystems A New Conservation Paradigm. Annals of the New York Academy of Sciences 1134:173-200.

Pfeiffer, M., D. Mezger, and J. Dyckmans. 2013. Trophic ecology of tropical leaf litter ants 
Hymenoptera : Formicidae ) - a stable isotope study in four types of Bornean rain forest Myrmecological News. 19:31-41.

Philpott, S. M., and I. Armbrecht. 2006. Biodiversity in tropical agroforests and the ecological role of ants and ant diversity in predatory function. Ecological Entomology 31:369-377.

Philpott, S. M., I. Perfecto, and J. Vandermeer. 2008. Effects of predatory ants on lower trophic levels across a gradient of coffee management complexity. Journal of Animal Ecology 77:505511.

Pinheiro, J., and D. Bates. 2014. Linear and Nonlinear Mixed Effects Models R-Package nlme.

R Core Team. 2014. R: A language and environment for statistical computing. R Foundation for Statistical Computing, Vienna, Austria:URL http://www.R-project.org/.

Rudolph, K. P., and T. M. Palmer. 2013. Carbohydrate as Fuel for Foraging, Resource Defense and Colony Growth - a Long-term Experiment with the Plant-ant Crematogaster nigriceps. Biotropica 45:620-627.

Sanders, D., and C. Platner. 2007. Intraguild interactions between spiders and ants and top-down control in a grassland food web. Oecologia 150:611-624.

Seifert, B. 2007. Die Ameisen Mittel- und Nordeuropas. lutra Verlags- und Vertriebsgesellschaft, Görlitz/Tauer.

Shattuck, S. O. 1992. Review of the Dolichoderinae ant genus Iridomyrmex Mayr with descriptions of three new gena (Hymenoptera: Formicidae). J. Aust. ent. Soc. 31:13-18.

Shattuck, S. O. 1994. Taxonomic catalog of the ant subfamilies Aneuretinae and Dolichoderinae (Hymenoptera: Formicidae). Univ. Calif. Publ. Entomol. 112:1-241.

Tillberg, C. V, D. a Holway, E. G. Lebrun, and A. V Suarez. 2007. Trophic ecology of invasive Argentine ants in their native and introduced ranges. Proceedings of the National Academy of Sciences of the United States of America 104:20856-20861.

Tillberg, C. V., D. P. McCarthy, A. G. Dolezal, and A. V. Suarez. 2006. Measuring the trophic ecology of ants using stable isotopes. Insectes Sociaux 53:65-69.

Tobin, J. E. 1991. A neotropical rainforest canopy, ant community: some ecological considerations. Pages 536-538 in C. R. Huxley and D. F. Cutler, editors. Ant-plant interactions. Oxford University Press, Incorporated, New York, NY.

Venables, W. N., and B. D. Ripley. 2002. Modern Applied Statistics with S. Springer, New York.

Vepsäläinen, K., and B. Pisarski. 1982. Assembly of island ant communities. Ann. Zool. Fennici 19:327-335.

Warren, R. J., and L. Chick. 2013. Upward ant distribution shift corresponds with minimum, not maximum, temperature tolerance. Global change biology 19:2082-8. 
Way, M. J., and K. L. Heong. 2009. Significance of the tropical fire ant Solenopsis geminata (hymenoptera: formicidae) as part of the natural enemy complex responsible for successful biological control of many tropical irrigated rice pests. Bulletin of Entomological Research research 99:503-512.

Wielgoss, A., T. Tscharntke, D. Buchori, B. Fiala, and Y. Clough. 2010. Temperature and a dominant dolichoderine ant species affect ant diversity in Indonesian cacao plantations. Agriculture, Ecosystems \& Environment 135:253-259.

Wielgoss, A., T. Tscharntke, A. Rumede, B. Fiala, H. Seidel, S. Shahabuddin, and Y. Clough. 2014. Interaction complexity matters: disentangling services and disservices of ant communities driving yield in tropical agroecosystems. Proceedings of the Royal Society B 281:20132144.

Wilson, E. O. 1971. The Insect Societies. Belknap of Harvard University Press. Cambridge.

Wood, S. 2000. Modelling and smoothing parameter estimation with multiple quadratic penalties. Journal of the Royal Statistical Society: Series B (Statistical Methodology) 62:413-428.

Woodcock, P., D. P. Edwards, R. J. Newton, F. A. Edwards, C. V. Khen, S. H. Bottrell, and K. C. Hamer. 2012. Assessing trophic position from nitrogen isotope ratios : effective calibration against spatially varying baselines. Naturwissenschaften 99:275-283. 
Supporting information - Chapter 3

$115 / 168$ 


\section{List S1 Present plant species (all plantations)}

Trees: Theobroma cacao (main crop), Gliricidia sepium (Leguminosae; shade and nitrogen fixation), Erythrina subumbrans (Leguminosae; shade and nitrogen fixation), Aleurites moluccana (Euphorbiaceae; candlenut, spice), Tectona grandis (Lamiaceae; wood production), Psidium guajava (Myrtaceae; common guava, fruits), Persea americana (Lauraceae; avocado, fruits), Nephelium lappaceum (Sapindaceae; rambutan, fruits), Mangifera odorata (Anacardiaceae; kuwini mango, fruits), Mangifera indica (Anacardiaceae; indian mango, fruits), Kaulero (local name, rope production), Durio zibethinus (Malvaceae; durian, fruits), Cocos nucifera (Arecaceae; coconut, fruits),Cinnamomum burmannii (Lauraceae; Indonesian cinnamon, spice), Bischofia javanica (Phyllanthaceae; bishop wood, wood production).

Intercrops: Arenga pinnata (Arecaceae; feather palm, sugar and wine production), Ananas comosus (Bromeliaceae; pineapple, fruits), Capsicum spp. (Solanaceae; chili, spice), Manihot esculenta (Euphorbiaceae; manioc, root), V anilla planifolia (Orchidaceae; vanilla, spice), Zea mays (Poaceae; maize, grain production).

Herbs: Ageratum conyzoides (Asteraceae; chick weed, weed), Spilanthes paniculata (Asteraceae; daungetang, weed), Paspalum conjugatum (Poaceae; buffalo grass, weed). 
Table S2 Fruit phenology categories.

\begin{tabular}{lllllllll}
\hline & \multicolumn{2}{c}{$\begin{array}{c}\text { Average } \\
\text { Fruit development classes }\end{array}$} & \multicolumn{2}{c}{$\begin{array}{c}\text { Average } \\
\text { width [cm] }\end{array}$} & $\begin{array}{c}\text { Groove depth } \\
{[\mathbf{m m}]}\end{array}$ & Colour & $\begin{array}{l}\text { Surface } \\
\text { structure }\end{array}$ \\
\hline & mean & SD & mean & SD & mean & SD & & \\
Small fruit & 5.3 & \pm 3.5 & 1.9 & \pm 1.2 & 0.1 & \pm 0.1 & green & Smooth \\
Medium fruit & 13.1 & \pm 3.0 & 5.7 & \pm 1.2 & 0.3 & \pm 0.1 & green & rough, uneven \\
Big fruit & 14.9 & \pm 3.3 & 7.5 & \pm 1.3 & 0.3 & \pm 0.1 & green, ripe spots & rough, uneven \\
Ripe fruit & 15.4 & \pm 3.4 & 7.9 & \pm 1.2 & 0.4 & \pm 0.1 & ripe: red or yellow & smooth, even \\
\hline
\end{tabular}




\section{Table S3 Ant colony size.}

(Categories and sums of individual of all 5 counted nests).

\begin{tabular}{llllll}
\hline Category & Species & Location & Workers & Queens & Larvae \\
\hline \multirow{4}{*}{1} & D. thoracicus & Napu & 1047 & 8 & 56 \\
& D. thoracicus & Palolo1 & 703 & 14 & 63 \\
& $P$. cf. cordata & Palolo2 & 708 & 3 & 33 \\
\hline \multirow{3}{*}{3} & D. thoracicus & Napu & 1470 & 12 & 431 \\
& D. thoracicus & Palolo1 & 1850 & 41 & 418 \\
& P. cf. cordata & Palolo2 & 1474 & 16 & 281 \\
\hline \multirow{3}{*}{4} & D. thoracicus & Napu & 3756 & 24 & 907 \\
& D. thoracicus & Palolo1 & 4945 & 74 & 1470 \\
& P. cf. cordata & Palolo2 & 4569 & 19 & 3354 \\
\hline & D. thoracicus & Napu & 46996 & 1315 & 15751 \\
& D. thoracicus & Palolo1 & 20455 & 196 & 8890 \\
& P. cf. cordata & Palolo2 & 15266 & 95 & 7609 \\
\hline
\end{tabular}

\section{Description S4 Pre-experiment life cycle $D$. thoracicus and $P$. cf. cordata}

We observed colony developments in plastic boxes with plaster ground and measured the days till the majority of eggs were hatched and no new workers appeared. The ant colonies were collected from the rearing plantations and a subset of separated ant nests was transferred to each of five boxes per species. Despite trying different approaches (colored diet, direct application of food dye, vanish, oil paint) the coloration failed or individually marked ant larvae disappeared (larvae predation or cleansing larvae). Therefore, observation of emerging new workers and disappearance of eggs was the only estimation opportunity. The transmigrated ant queens did not produce new eggs, but transferred eggs hatched and newly emerged ant workers were colored lighter than the transferred ones. After four weeks, all eggs were hatched. We observed a similar development span for $D$. thoracicus and $P$. cf. cordata. 
Chapter 4: Multi-scale intensification changes trophic position of omnivores in tropical agroforestry

Pierre Gras, Teja Tscharntke, Yann Clough

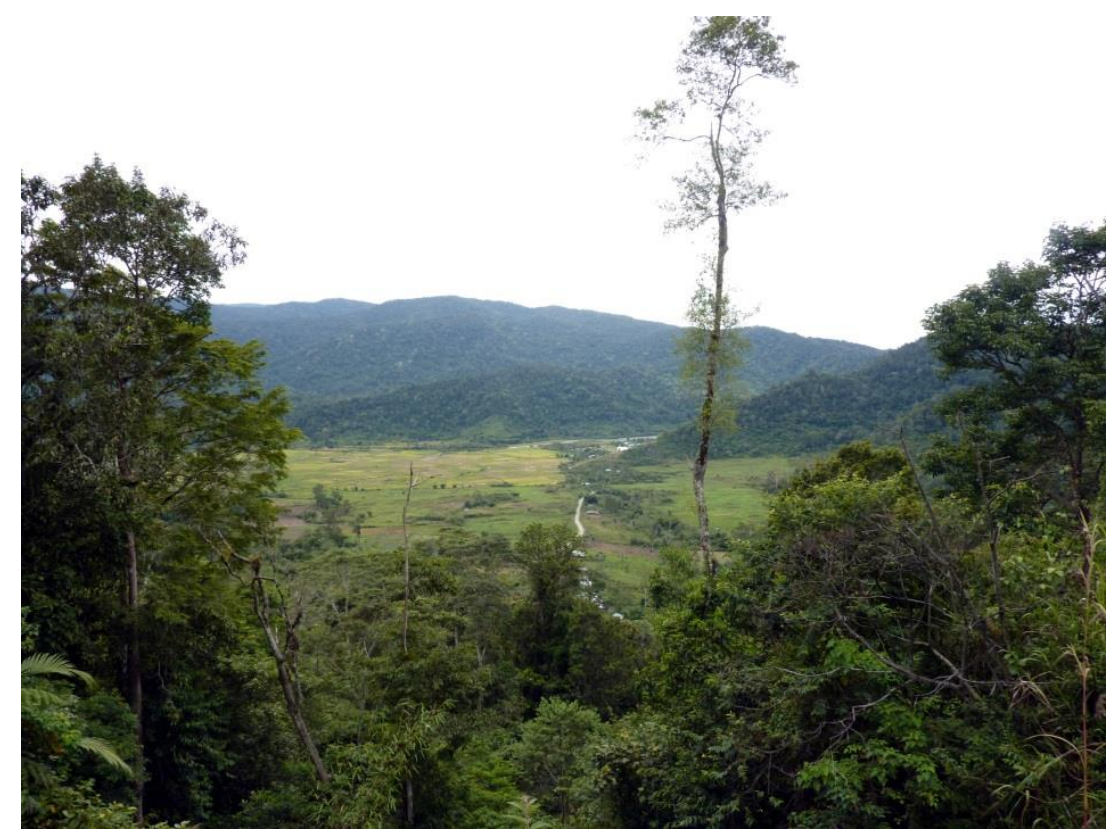

The Napu valley (picture from the forest margin).

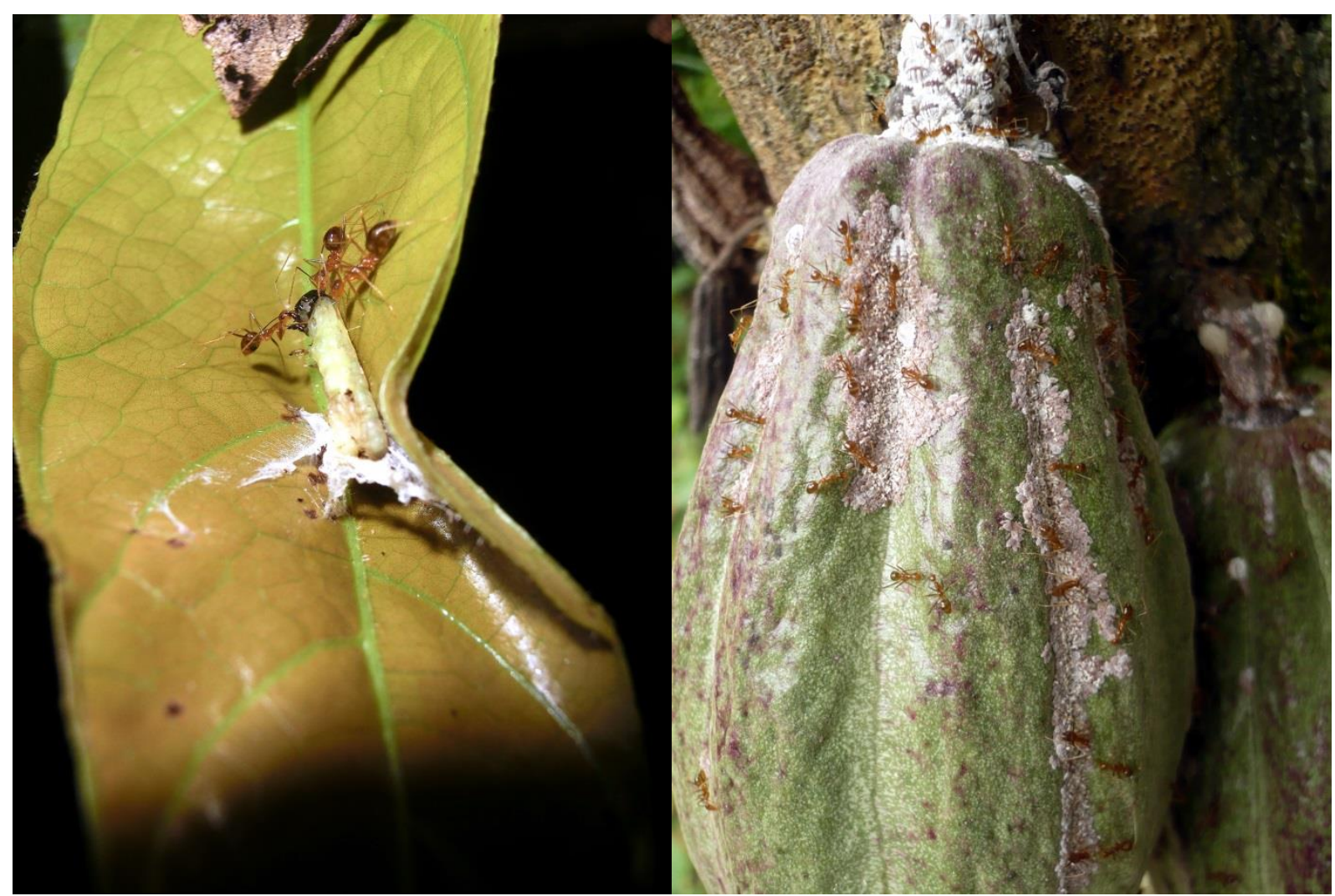

Anoplolepis gracilipes captures a freshly pupated, leaf-rolling caterpillar (left) and $A$. gracilipes obtains honeydew of trophobionts (Cataenococcus hispidus Morrison (Hemiptera: Pseudococcidae)).

Manuscript prepared for: Oecologia (status: not yet submitted) 


\section{$120 / 168$}




\section{Summary}

Omnivores can stabilise trophic webs through preferential consumption of the more abundant resources. However, it is unclear how species-turnover and trophic plasticity contributing to the variability, or conversely the stability, in the trophic role of communities of omnivores across environmental gradients.

Here, we test whether local vegetation structure (shade tree cover) and landscape context influence the trophic position of ants in agroforests, and whether these changes occur within species or due to a change in community composition. We sampled ants in 15 cacao agroforests, differing in shade and distance to natural forest, and used stable isotope analyses ( $\delta 15 \mathrm{~N}$ and $\delta 13 \mathrm{C}$ ) to quantify resource-use. We found that trophic levels occupied by ant communities decreased under high intensification (low shade tree cover and far from forest margins). Trophic positions of particular omnivorous ants show similar patterns (P. dives, C. reticulatus, M. floricola and T. albipes), while other species maintained stable tropic positions along both gradients (A. gracilipes, T. pacificum, O. simillimus). Intraspecific trophic positions - at least partly - influenced the spatial distribution ant species in cacao agroforestry.

We infer that both, trophic plasticity and species turnover, are mechanisms defining the predatory functions of ant communities. As some ant species adapt tropic positions depending on local management and landscape context, while others maintain stable trophic levels. Despite that, strong disturbance at both scales can lower the predatory function of ant communities. Thus, we infer detailed ecological knowledge at species level is mandatory to identify the role of mesopredators such as ants in ecosystem services provision. 


\section{Introduction}

Omnivory is a major determinant of food web stability and population dynamics (Singer and Bernays 2003; Thompson et al. 2007; Pocock et al. 2012). It is unclear, however, how speciesturnover and trophic plasticity contribut to the variability, or conversely the stability, in the trophic role of communities of omnivores across environmental gradients. This question is not only of general ecological interest, but also of applied interest since omnivores are often important in regulating pest organisms. Progress in studying dietary changes of omnivores with community or environmental context has been slow for methodological limitations, but the assessment of trophic positions by stable isotope analysis has emerged as powerful tool to study trophic elasticity in terrestrial ecosystems (Ponsard and Arditi 2000; Davidson et al. 2003; Ottonetti et al. 2008; Woodcock et al. 2013).

Ants cover a broad foraging spectrum ranging from cryptic herbivory to predation (Hölldobler and Wilson 1990; Folgarait 1998; Philpott and Armbrecht 2006; Philpott et al. 2008). Many ant species both tend plant-sucking trophobionts (such as aphids or scale insects) to collect carbohydrate-rich honeydew, and prey or scavenge on arthropods and other animal resources to access nitrogen-rich resources (Floren et al. 2002). The trophic levels of ants vary between species, with strong differences between subfamilies (Davidson et al. 2003). Ant species turnover, as has been shown to occur in response to land-use change (Rizali et al. 2012, see Chapter 2) could thus potentially be associated with community-level shift in trophic position (Pfeiffer et al. 2003). However, one may also expect significant variability in trophic position within species or local populations (Tillberg et al. 2006, Blüthgen et al. 2003), as ant colonies adapt to shifts in the relative availability of different resources.

In tropical agroforests, ants play an important role in protecting plants from pests (Vandermeer et al. 2002; Way and Heong 2009; Mollot et al. 2012; Offenberg et al. 2013; Gonthier et al. 2013; Wielgoss et al. 2014). Management practices of agroforests vary greatly and structure different microhabitats, while the diversity of insect communities' declines with land-use intensification (Armbrecht et al. 2005; Philpott et al. 2006). For example, intensively managed suncacao plantations provide a hot and dry microclimate with few nesting-sites or food resources leading to insect communities dominated by few competitively dominant species including $A$. gracilipes (Rice and Greenberg 2000b; Philpott and Foster 2005; Armbrecht et al. 2006; Bos et al. 2008, personal observation PG). Shade tree removal negatively affects arboreal ants in particular, since they depend on intact canopy structures (Floren et al. 2002; Kone et al. 2012; Floren et al. 2014). Further, increasing distance from rain forest patches affect the composition of arthropod communities (Klein et al 2006, Clough 2010). 
We used stable isotope analysis to examine the relationship of omnivores' resource-use and land-use change in a well-studied cacao agroforestry system in Sulawesi, Indonesia. We tested patterns of worker abundance, species diversity and carbon and nitrogen isotope signatures of ant communities, and in addition, seven omnivorous ant species: We conducted stable isotope based analyses, as the natural isotopic composition of nitrogen $\left(\delta^{15} \mathrm{~N}\right)$ or carbon $\left(\delta^{13} \mathrm{C}\right)$ - a ratio of heavier to lighter isotopes - varies between organisms due to fractionation against heavier isotopes in respiratory processes. In trophic web research, this mechanism is utilized to track down food energy flow and source dependency in habitat-specific trophic webs, since heavy carbon and nitrogen isotopes accumulate within the body, depending on the digested diets (Blüthgen et al. 2003; Ottonetti et al. 2008; International Atomic Energy Agency 2009).

We ask the following questions:

1. Do differences in shade tree cover and distance to forest margins affect the community-level trophic position of omnivores?

2. Is the variability - or stability - observed at community-level due to species turnover or changes in species-specific diets along these two gradients?

\section{Material and methods}

\section{Study sites}

In June 2012, we sampled ants, spiders and cacao leaves in 15 smallholder cacao agroforests at the eastern border of the Lore Lindu National Park in Central Sulawesi, Indonesia $\left(1^{\circ} 25^{\prime} 31.8^{\prime \prime}\right.$ $\left.\mathrm{S}, 120^{\circ} 18^{\prime} 57.55^{\prime \prime} \mathrm{E}\right)$. The agroforests were located in the northern part of the Napu valley at $\sim 1130 \mathrm{~m}$ a.s.l. and rainfall between $1990 \mathrm{~mm}$ and $3804 \mathrm{~mm}$ (Fig. 1). Each agroforest covered 0.5 to 2 ha and we established an experimental core plot $\left(\sim 225 \mathrm{~m}^{2}\right.$, five-times-five tree grid, $3 \mathrm{~m}$ intertree distance) covering 25 cacao trees in the centre. Local farmers managed all agroforests, but we implemented pesticide free areas $(>15 \mathrm{~m}$ radius, connected with 2 agroforest edges) to minimize management differences. All plantations were separated by at least $500 \mathrm{~m}$ (edge-to-edge distance). The non-crop plant community mainly consisted of two planted legume shade trees (Erythrina subumbrans MERR and Gliricidia sepium KUNTH), representing 30 - $91 \%$ of all non-crop trees (Table S1, herbal plants see Table S2). The herb layer was trimmed every two months with a motor scythe. 


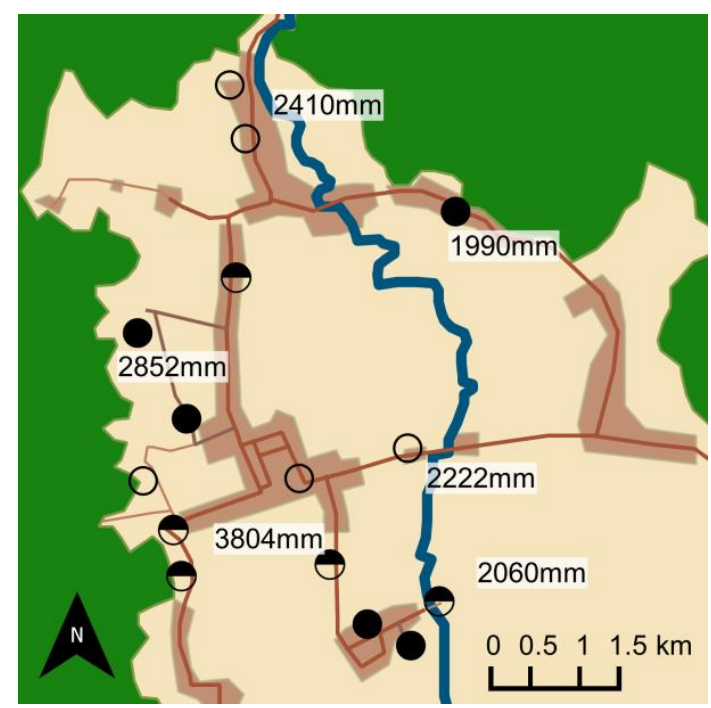

Fig. 1. Study area in the Napu valley of Central Sulawesi, Indonesia. Cycles show the location of the agroforests, filling style shows the canopy cover (empty $<30 \%$, half $=30-50 \%$, filled $>50 \%$ ), white labels show regional rainfall in $\mathrm{mm}$, green areas at the map borders indicate rainforest, reddish areas houses, bright areas open land and lines streets.

\section{Sampling and analysing methods}

General approach. All 15 plantations situated along the canopy cover and the forest distance gradient were sampled once between May 2012 and June 2012. We sampled ants, spiders and cacao leaves within each established 25 tree plot in two hours. One person sampled ants and two person spiders as well as cacao leaves.

Sampling of arthropods for stable isotope analysis. We sampled ants (omnivores) and spiders (exclusive predators) using manual searching and canopy sweep netting at cacao tree surfaces (leaves, flowers, fruits). Each tree was scanned from the top to the ground, whereby we started in the centre of each plot extending the search towards the edges until each tree as well as the ground below each tree was scanned. To handle samples latex gloves were obligatory. Each captured individual was put into a two millilitre Eppendorf tube and killed by freezing less than five hours after sampling. Ant samples were identified with taxonomic literature and regional collections of Arno Wielgoss and Akhmad Rizali (Bolton 1994; Fisher 2010; Wielgoss et al. 2010; Rizali et al. 2010; Rizali et al. 2013). If available, we arranged ten ants of each taxon in a master collection deposited and verified by Akhmad Rizali at the Bogor Agricultural University.

Sampling of plant material for stable isotope analysis. We sampled leafs of three individuals of Theobroma cacao by cutting $4 \mathrm{~cm}^{2}$ leaf tissue from each of two mature shade-growing leaves, all originating from different shoots.

Processing of samples for stable isotope analysis. To handle samples, not powdered latex gloves were obligatory. In the study region, all collected samples were stored in a freezer (-18 ${ }^{\circ} \mathrm{C}$ ) and transported to Tadulako University (Palu, Central Sulawesi). We kept all samples frozen 
until drying in a ventilated drying cabinet. Dry samples were stored in Eppendorf tubes, put in plastic bags and embedded in silica gel within hermetic proofed plastic boxes. In December 2012, the samples arrived at the Georg-August Universität Göttingen. We replaced the silica gel despite still dry and stored all samples in a dark and dry environment at $16-18^{\circ} \mathrm{C}$.

Stable isotope analyses. We used the heads of ant individuals, milled the leaf tissue using an electronic mill (Janke \& Kunkel, tabletop hammer mill type: MFC, $5000 \mathrm{r} / \mathrm{min}$ ), and pestled whole-bodied spiders. We filled 0.5 to $1 \mathrm{mg}$ of animal tissue or $1-2 \mathrm{mg}$ of plant tissue (Sartorius micro, $0.001-3.000 \mathrm{mg}, \mathrm{d}<0.001 \mathrm{mg})$ in tin capsules $(5 \times 8 \mathrm{~mm})$. This was based on the expected and pretested order of magnitude of the nitrogen content. Stable isotope measurements were conducted by isotope mass spectrometry (Delta+, Termo Finnigan) coupled to an elemental analyser (NA1110, CEE - Instruments). The stable isotope analyses were conducted at the Centre for Stable Isotope Research and Analysis (KOSI) at the University of Göttingen. We received ready-to-use data tables reporting the $\delta^{15} \mathrm{~N}$ and $\delta^{13} \mathrm{C}$ signatures.

Selection of ant species. To test trophic shifts of particular ant species, we selected ant species present on more than 8 agroforests at different levels of canopy cover and forest distance. (1) Monomorium floricola (JERDON, 1851) 35 samples from 15 plantations, (2) Odontomachus simillimus (SMITH, 1858) 12 samples from 23 plantations, (3) Tetramorium pacificum (MAYR, 1870) 22 samples from 12 plantations, (4) Technomyrmex albipes (SMITH, 1861) 35 samples from 15 plantations, (5) Anoplolepis gracilipes (SMITH, 1857) 15 samples from 8 plantations, (6) Polyrhachis dives (SMITH, 1857) 16 samples from 9 plantations, and (7) Camponotus reticulatus (ROGER, 1863) 16 samples from 11 plantations. All these seven species have been recorded at more than 8 plantations, and were selected to represent intraspecific variability of stable nitrogen isotopes (average value over all samples of all sites of one ant species) over the gradients.

Ant abundance estimation. To estimate the amount of ants present at each plantation, we used presence/absence values of all found ant species distributed over all plantations.

\section{Statistical analyses}

To analyse the data, we proceeded in three steps. First, we conducted a pre-analysis framing the variation of isotopic signatures using the baseline signal (cacao leaves) and samples of exclusive predators (spiders) along the canopy cover and forest distance gradients. Second, - to test the first hypothesis - we applied the method described below to analyse a data set containing all present ant taxa (48 morpho species) to testing pattern for the whole community. Third, - to test the second hypothesis - we used the same method testing patterns for the seven most common omnivorous ant species (A. gracilipes, T. albipes, O. simillimus, P. dives, C. reticulatus, T. pacificum, and M. floricola) recorded in more than 8 agroforests along both gradients. 
Step 1 pre-analysis. We fitted linear mixed-effects (LME) models containing the following variables: (1) $\delta^{15} \mathrm{~N}$ or $\delta^{13} \mathrm{C}$ signatures of cacao leaves as response variable, (2) a categorical predictor for shade showing three levels of canopy cover (low $=0-30 \%$, med $=31-50 \%$, high $=>50 \%$ ), (3) the calculated shortest distance between plantation centre and rainforest margin, (4) the squared distance from the plantation centre to the rainforest margin to model non-linearity, (5) interaction of shade and forest distance, (6) plantation as random effect. We fitted the same models to the isotope values of the spiders. We conducted standard model validation (Pinheiro and Bates 2014), and compared the information content (AIC) of models with and without the variance function. If necessary, we included a variance function (power-function) to meet assumptions of heteroscedasticity. Additionally, the model fit was evaluated by graphically examining variance inflation factors, residuals vs. fitted values, and normal QQ-plots. Finally, we conducted backwards stepwise model selection based on AIC (R-function "stepAIC"), and reported the model with the lowest AIC. Additionally, the unstandardized $\delta^{15} \mathrm{~N}$ and $\delta^{13} \mathrm{C}$ signatures of ant species, spiders and cacao leaves were inspected graphically (Fig S3, S4 and S5).

Step 2 Ant community analyses of stable-isotope signatures. To correct for spatial variation of the isotopic baseline signal due to not manipulated, plantation-specific attributes (e.g. soil type or humidity, fertilization), we standardised $\delta^{15} \mathrm{~N}$ and the $\delta^{13} \mathrm{C}$ signatures of ants by subtracting mean values of cacao leaves. We applied small scale standardisation (see Woodcock et al. 2012) and the sampling was restricted to a 12 times $12 \mathrm{~m}$ plot within homogeneous agroforests. We then used the same LME modelling approach described above, but used standardised $\delta^{15} \mathrm{~N}$ as well as standardised $\delta^{13} \mathrm{C}$ signatures of ant individuals as response variable and the species was nested in plantation as random structure.

Step 3 Omnivorous ant species analyses of stable isotope-signatures. Here we repeated the analysis done in step two restricted to the selected seven omnivorous ants (see above).

Step 4 Analyses of the relationship of ant species identity, stable isotope-signatures, shade and forest distance. In a separate modelling approach, we tested how the presence of different ant species relates to the isotopic signature, canopy cover and forest distance. To do so, logistic regressions were conducted separately for all ant species and for omnivorous ants only. The models contain the following variables: (1) a binomial vector coding ant species presence/absence as response variable, (2.1) intraspecific $\delta^{15} \mathrm{~N}$ signatures (mean value of all analysed individuals of one species) or (2.2) intraspecific $\delta^{13} \mathrm{C}$ signatures (mean value of all analysed individuals of one species), (3) a categorical predictor for shade showing three levels of canopy cover (low $=0-30 \%$, med $=31-50 \%$, high $=>50 \%)$, (4) the calculated shortest distance between plantation centre and rainforest margin, (5) the squared distance from the plantation centre to the rainforest margin to model non-linearity, (6) interaction of shade, forest distance and intraspecific $\delta^{15} \mathrm{~N}$ signatures, (7) 
interaction of shade, forest distance and intraspecific $\delta^{13} \mathrm{C}$ signatures, and (8) plantation as random effect. Both models have been generalized linear mixed models fitted via PQL, we graphically examined the model fit and computed p-values (ANOVA Type 2) as indicator for significant interactions and relationships.

All statistical analyses were performed in R 3.03 (R Core Team 2014). We used the packages: nlme, MASS, splines, and effects (Wood 2000; Venables and Ripley 2002; Fox 2003; Oksanen et al. 2013; Pinheiro and Bates 2014; R Core Team 2014).

\section{Results}

We recorded 48 different ant (morpho-) species (Table S4) in all 15 agroforests.

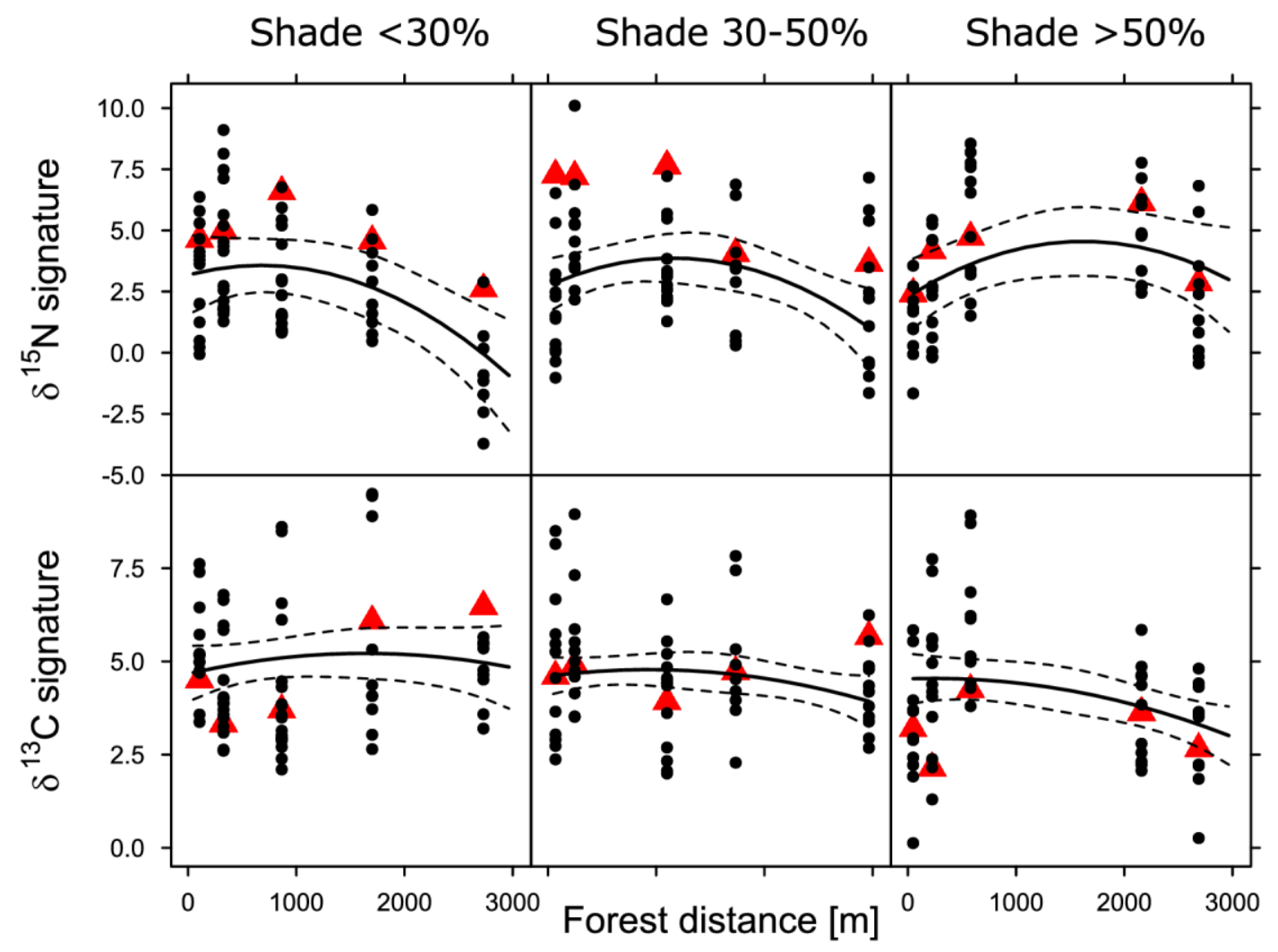

Fig. 2. Standardised $\delta^{15} \mathrm{~N}$ and $\delta^{13} \mathrm{C}$ signature of all analysed ant species, spiders and cacao samples in response to forest distance and shade cover. Black dots $=$ ants, red triangles $=$ spiders. Statistics: $\delta^{15} \mathrm{~N}$ : Optimum shape of forest distance $(\mathrm{P}=0.02)$ and interaction shade-forest distance $(\mathrm{P}=0.02)$ were significant different, $\delta^{13} \mathrm{C}$ : different levels of shade tended to be different $(\mathrm{P}=0.09)$.

The distance between primary producers (T. cacao, isotopic signature: $0 \delta^{15} \mathrm{~N}$ ) and ants varied between 9 deltas within one local community (Fig. 2), a distance reflecting three to four trophic levels as different consumer levels are separated by $2.3-3.0 \delta^{15} \mathrm{~N}$ (Hood-Nowotny and Knols 2007; International Atomic Energy Agency 2009). The standardised $\delta^{15} \mathrm{~N}$ signatures of ant communities peaked at medium distance to the forest margin and dropped far from the forest 
margins under less than 30\% canopy cover $\left(\mathrm{F}\right.$ shade $(1,10)=0.87, \mathrm{P}=0.37 ; \mathrm{F}_{\text {Forest distance }(1,10)}=1.88$, $\left.\mathrm{P}=0.20 ; \mathrm{F}_{\text {Forest distance } 2(1,10)}=4.97, \mathrm{P}=0.05 ; \mathrm{F}_{\text {Interaction-shade-forest distance }(1,10)}=4.90, \mathrm{P}=0.05\right)$. The standardised $\delta^{13} \mathrm{C}$ signatures of the ant communities tended to differ between shade cover $(\mathrm{F}$ shade $\left.{ }_{(1,13)}=3.46, \mathrm{P}=0.09\right)$. Standardised $\delta^{15} \mathrm{~N}$ values of spiders peaked under medium shade, but forest distance did not matter $\left(\mathrm{F}_{\text {Shade }(1,11)}=7.96, \mathrm{P}=0.02 ; \mathrm{F}_{\text {Forest distance }(1,11)}=0.23, \mathrm{P}=0.64 ; \mathrm{F}_{\text {Interaction }(1,11)}\right.$ $=2.45, \mathrm{P}=0.15)$. The standardised $\delta^{13} \mathrm{C}$ signatures dropped linear with increasing shade and tended to increase with forest distance $\left(\mathrm{F}_{\text {Shade }}(1,12)=11.14, \mathrm{P}<0.01 ; \mathrm{F}_{\text {Forest distance }(1,12)}=15.37, \mathrm{P}<0.01\right)$.

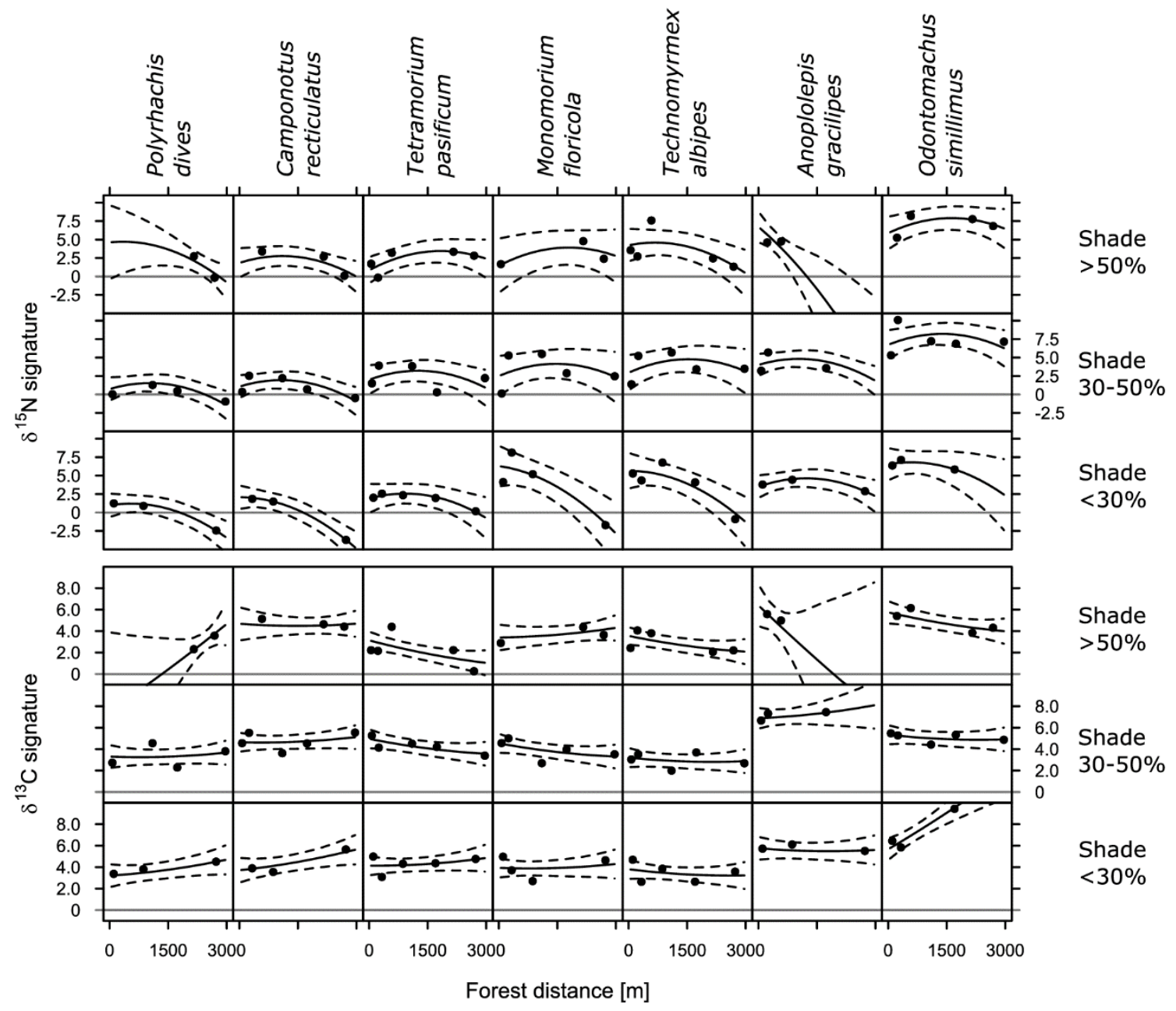

Fig. 3. Interactive effect of canopy cover and forest distance on $\delta^{15} \mathrm{~N}$ and $\delta^{13} \mathrm{C}$ signatures of omnivorous ants. The continuous lines show the estimated mean effect size, the dashed lines indicate $95 \%$ confidence intervals, the grey line indicates 0 .

Analysing the standardised isotopic signatures of seven omnivorous ant species A. gracilipes, T. albipes, O. simillimus, P. dives, C. reticulatus, T. pacificum, and M. floricola revealed that (Fig. 3): (1) standardised $\delta^{15} \mathrm{~N}$ variation was species-specific, each interspecific trophic position was modelled by interactive effects of shade and forest distance and tended to show the highest values at intermediate forest distances $\left(\mathrm{F}_{\text {Species }(6,31)}=362.74, \mathrm{P}<0.01 ; \mathrm{F}_{\text {Forest distance }(1,8)}=1.99, \mathrm{P}=0.20\right.$; 
$\mathrm{F}_{\text {Shade }(2,8)}=0.45, \mathrm{P}=0.66 ; \quad \mathrm{F}_{\text {Forest distance }^{\wedge}(1,8)}=3.64, \quad \mathrm{P}=0.09 ; \quad \mathrm{F}_{\text {Species-forest distance }(6,31)}=28.20$, $\mathrm{P}<0.01 ; \mathrm{F}_{\text {Species-shade }(12,31)}=4.73, \mathrm{P}<0.01 ; \mathrm{F}_{\text {Forest distance-shade }(2,8)}=0.37, \mathrm{P}=0.64 ; \mathrm{F}_{\text {Species-forest distance- }}$ shade $(12,31)=8.44, \mathrm{P}<0.01)$. (2) Similarly, the variation in standardised $\delta^{13} \mathrm{C}$ was species-specific, shade dependent and species-specific carbon signatures were modelled by interactive effects of shade and forest distance $\left(\mathrm{F}_{\text {species }(6,31)}=56.14, \mathrm{P}<0.01 ; \mathrm{F}_{\text {Forest distance }(1,9)}=0.86, \mathrm{P}=0.38 ; \mathrm{F}_{\text {Shade }}\right.$ ${ }_{(2,9)}=7.56, \quad \mathrm{P}=0.01 ; \quad \mathrm{F}$ Species-forest distance $(6,31)=1.79, \mathrm{P}=0.14 ; \quad \mathrm{F}$ Species-shade $(12,31)=6.3, \mathrm{P}<0.01 ;$ $\left.\mathrm{F}_{\text {Forest distance-shade (2,9) }}=4.14, \mathrm{P}=0.05 ; \mathrm{F}_{\text {Species-forest distance-shade (12,31) }}=4.59, \mathrm{P}<0.01\right)$.

In detail: (1) Nitrogen signatures (Fig. 3, top half): A. gracilipes and O. simillimus showed the highest values with 5-7 $\delta^{15} \mathrm{~N}$. The signatures remained relatively stable throughout all levels of shade and all forest distances showing highest values for intermediate forest distances. Shown signatures of ants covered tropic levels typical for herbivores, predators and intra-guild predators. P. dives, $C$. reticulatus, T. pacificum, $M$. floricola, and T. albipes showed intermediate nitrogen signatures (between 0 and $5 \delta^{15} \mathrm{~N}$, increasing left to right), whereby the values dropped for approximately three deltas with increasing forest distance under low-canopy cover. (2) Carbon signatures (Fig. 3, bottom half): A. gracilipes and O. simillimus showed the highest values with $6-9 \delta^{13} \mathrm{C}$. P. dives, C. reticulatus, T. pacificum, M. floricola, and T. albipes showed values between 3 and $5 \delta^{13} \mathrm{C}$. The signatures remained stable for all forest distances, but decreased with increasing shade for about 1 delta. Variation of the species-specific nitrogen signature was shown for $P$. dives and $A$. gracilipes under low shade as well as for O. simillimus under high shade cover. In all cases, only two or three samples were present instead of five.

Finally, we tested how presence/absence of ant species in different agroforests related to isotopic signatures, shade cover and forest distance (Table 1 and 2). The fitted logistic regression indicated that species rich ant communities showed higher intraspecific $\delta^{15} \mathrm{~N}$ signatures than species poor ant communities (Table $1, F=7.72 \mathrm{P}=0.01$ ). The same analysis restricted to omnivorous ant species illustrated an interactive influence of forest distance, as only for sites close to the forest margin increasing numbers of omnivorous ant species resulted in higher intraspecific $\delta^{15} \mathrm{~N}$ signatures $\left(\mathrm{F}_{\text {Forest distance- int. } 815 \mathrm{~N} \text { signature }}=3.43, \mathrm{P}=0.06\right)$. The intraspecific $\delta^{13} \mathrm{C}$ signatures were not related to the number of locally presence ant species (Table 2 ).

The isotopic base line signature (cacao leaves) showed low $\delta^{15} \mathrm{~N}$ values close to forest margins and under high shade cover, most likely due to the presence of legume shade trees $(\mathrm{F}$ shade ${ }_{(2,9)}=4.26, \mathrm{P}=0.05 ; \mathrm{F}_{\text {Forest distance }(1,9)}=10.40, \mathrm{P}=0.01 ; \mathrm{F}$ Shade-forest distance $\left.(2,9)=4.14, \mathrm{P}=0.05\right)$. The $\delta^{13} \mathrm{C}$ values showed a strong variation for each plantation, but not for shade or forest distance $\left(\mathrm{F}_{\text {Shade }(2,9)}=2.0, \mathrm{P}=0.19 ; \mathrm{F}_{\text {Forest distance }(1,9)}=0.73, \mathrm{P}=0.41 ; \mathrm{F}_{\text {Shade-forest distance }(2,9)}=2.23, \mathrm{P}=0.16\right)$. 
Table 1 ANOVA type II relationship of ant species presence, shade, forest distance, intraspecific $\delta 15 \mathrm{~N}$ signatures. '-' = interacting with, '*' $=$ statistical significant and $'$ ' = marginal statistical significant.

\begin{tabular}{|c|c|c|c|c|c|c|c|}
\hline & \multicolumn{3}{|c|}{ Ant community } & & \multicolumn{3}{|c|}{ omnivorous ant species } \\
\hline & Chisq & Df & $\operatorname{Pr}(>$ Chisq $)$ & & Chisq & Df & $\operatorname{Pr}(>$ Chisq $)$ \\
\hline Shade & 1.24 & 2 & 0.54 & & 4.04 & 2 & 0.13 \\
\hline Forest distance & 0.01 & 1 & 0.92 & & 0.07 & 1 & 0.79 \\
\hline Intraspecific (int.) $\delta^{15} \mathrm{~N}$ signature & 7.72 & 1 & 0.01 & $*$ & 0.89 & 1 & 0.35 \\
\hline Forest distrance^2 & 0.51 & 1 & 0.48 & & 0.64 & 1 & 0.43 \\
\hline Shade-forest distance & 1.59 & 2 & 0.45 & & 3.38 & 2 & 0.18 \\
\hline Shade- int. $\delta 15 \mathrm{~N}$ signature & 0.88 & 2 & 0.65 & & 0.47 & 2 & 0.79 \\
\hline Forest distance- int. $\delta 15 \mathrm{~N}$ signature & 1.03 & 1 & 0.31 & & 3.43 & 1 & 0.06 \\
\hline Shade- F. distance- int. $\delta 15 \mathrm{~N}$ signature & 3.11 & 2 & 0.21 & & 0.38 & 2 & 0.83 \\
\hline
\end{tabular}

Table 2 ANOVA type II relationship of ant species presence, shade, forest distance, intraspecific $\delta^{13} \mathrm{C}$ signatures. '-' indicates an interaction, '*' indicates statistical significant, '. indicates marginal statistical significant.

\begin{tabular}{|c|c|c|c|c|c|c|}
\hline & \multicolumn{3}{|c|}{ Ant community } & \multicolumn{3}{|c|}{ omnivorous ant species } \\
\hline & Chisq & Df & $\operatorname{Pr}(>$ Chisq $)$ & Chisq & Df & $\operatorname{Pr}(>$ Chisq $)$ \\
\hline Shade & 1.27 & 2 & 0.53 & 4.75 & 2 & 0.09 \\
\hline Forest distance & 0.02 & 1 & 0.88 & 0.10 & 1 & 0.76 \\
\hline Intraspecific (int.) $\delta^{13} \mathrm{C}$ signature & 1.86 & 1 & 0.17 & 1.26 & 1 & 0.26 \\
\hline forest distrance ${ }^{\wedge} 2$ & 0.44 & 1 & 0.51 & 1.91 & 1 & 0.17 \\
\hline Shade-forest distance & 1.53 & 2 & 0.47 & 2.59 & 2 & 0.27 \\
\hline Shade- int. $813 \mathrm{C}$ signature & 0.24 & 2 & 0.89 & 0.22 & 2 & 0.90 \\
\hline forest distance- int. $\delta 13 \mathrm{C}$ signature & 0.02 & 1 & 0.90 & 2.37 & 1 & 0.12 \\
\hline Shade-F. distance- int. $\delta 13 \mathrm{C}$ signature & 0.53 & 2 & 0.77 & 2.67 & 2 & 0.26 \\
\hline
\end{tabular}

\section{Discussion}

Within ant communities, the resource-use of omnivorous ants differed compared to the whole ant community. The $\delta^{15} \mathrm{~N}$ signature of the ant community dropped with increasing distance to the forest margin at low shaded shade plantations. The same pattern was true for the omnivores P. dives, C. reticulatus, M. floricola, and T. albipes, but A. gracilipes, T. pacificum and O. simillimus showed stable signatures at all sites. Increasing numbers of locally present ant species were positively correlated with the intraspecific nitrogen signature. Interestingly, the intraspecific nitrogen signatures explained the number of present particular ant species at plantations close to the rainforest margin only. The spatial distribution of ant species was independent of intraspecific carbon signatures.

As the $\delta^{15} \mathrm{~N}$ values of cacao leaves decreased with increasing shade cover, small-scale standardisation was necessary to reveal changes, concealed due to high variation in isotopic signatures at different locations (Woodcock et al. 2012; Pfeiffer et al. 2013). Beside environmental 
influences (e.g. drought or soil type), the changing baseline signature could be caused by increasing amounts of legume shade tree litter with increasing canopy cover, as shade trees (G. sepium and E. subumbrans) showed values approximately $2 \delta^{15} \mathrm{~N}$ lower than cacao leaves (unpublished data PG).

Ant communities exhibit high stability in nitrogen and carbon resource-use across environmental gradients, potentially due to changes of community composition and intraspecific resource-use. For example, Bornean leaf litter ants occupy trophic niches as herbivores, omnivores and true predators in a wide range of different rain forest types, with species-specific trophic functions (Pfeiffer et al. 2013). Truly predacious and abundant arboreal ants are restricted to rainforests or forest-like, diverse agroforests, while abundant and omnivorous ant species are evenly distributed (Kone et al. 2012). In our study, nitrogen signatures of ant communities dropped with increasing forest distance at low shaded plantations only. Such a pattern indicates that dense canopies provided either more arthropod prey or ants preyed at higher trophic levels. Both meets expectations as arthropods are more abundant in diverse, well-shaded or richly structured agroecosystems (Bos et al. 2007; Meijer et al. 2010; Mitchell et al. 2014; Kone et al. 2014). Thus, increased prey availability could have caused higher $\delta^{15} \mathrm{~N}$ signatures of larvae and adults as well as numbers of feeding on body tissue of non-ant predators such as spiders, wasps, earwigs (Bisseleua et al. 2013; Kone et al. 2014). In agroforests far away from forest margins and with low similarity to natural forests, abundances of arthropods generally decrease (Bos et al. 2007; Clough et al. 2010 ; Meijer et al. 2010; Mitchell et al. 2014). Thus, diets of ants might be protein poor when little shade was available and distance to rainforests was high, a pattern indicated by community wide low nitrogen signatures.

The carbon signature of ant communities increased when shade decreased. Arboreal ants depend on carbohydrates derived from plant sources (nectar, honeydew) to maintain metabolism and biomass (Tobin 1991). Dropping values with increasing shade cover can indicate use of resources such as maize or $\mathrm{C} 4$ grasses having $\delta^{13} \mathrm{C}$ signatures above cacao (Fry 2006; Girard et al. 2011; Madeira et al. 2014). A very likely explanation, as such plants directly benefit of light-flooded canopies.

At ant community level, we could show that for the community the average of intraspecific trophic levels $\left(\delta^{15} \mathrm{~N}\right)$ increased when more ant species were present. A result well supported by literature as high ant diversity is often accompanied by increasing functional diversity (Philpott and Armbrecht 2006; Wielgoss 2014). Interestingly, evenly spread ant species showed only in well-shaded agroforests close to the rainforest margins a similar pattern. Such close-to-nature habitats are known to be rich in biodiversity and provide many herbal and animal resources (Bos et al. 2007; Clough et al. 2010; Meijer et al. 2010; Mitchell et al. 2014). While the intraspecific trophic 
level was strongly related to the spatial ant distribution, the intraspecific carbon $(813 \mathrm{C})$ signatures were not.

Omnivores are generally supposed to exceed stabilising effects on species communities and trophic webs (Singer and Bernays 2003; Pocock et al. 2012). For our study system, we infer that omnivores exhibit species-specific trophic roles. Omnivorous ants such as $P$. dives, C. reticulatus, $M$. floricola and T. albipes, adapt their food mix in highly anthropogenic habitats providing little shade and being far from the forest margins. On the other hand, A. gracilipes, T. pacificum and O. simillimus maintained stable tropic positions at all sites. The nitrogen signatures of adult ants are determined by protein-rich larval diets (Vinson 1968; Haack et al. 1995). Omnivores are supposed to favour abundant resources switching between resources when one becomes scarce (Naranjo and Gibson 1996; Fagan 1997; Eubanks and Denno 1999) or capture prey of different trophic levels (Rosenheim 2001). With less herbivores available ant species such as O. simillimus $\left(\sim 7 \delta^{15} \mathrm{~N}\right)$ could maintain a high trophic level by either preying on other predators (including ants), or exclude competitors from resources by for example territorial behaviour (Arnan et al. 2011). Relatively high $\delta^{15} \mathrm{~N}$ signatures of $O$. simillimus are typical for second order predators. Moreover, this species is equipped with very strong mandibles and known for cannibalism and egg predation (Van Walsum et al. 1998). A. gracilipes can forage aggressively, but - according to its isotopic signature - captured prey similar to first-order predators $\left(5 \delta^{15} \mathrm{~N}\right)$. This is not surprising as $A$. gracilipes is known to dominate insect communities of agroforests, displacing other species, maintaining trophobiosis and preying on various herbivorous arthropods (Bos et al. 2008). The ecology of T. pacificum is widely unknown, it shows similar isotopic signatures as $A$. gracilipes and O. simillimus. Nitrogen signatures of $P$. dives, C. reticulatus, $M$. floricola and T. albipes dropped with increasing forest distance at low shade plantations, but showed relatively stable trophic positions in high and medium shaded agroforests. T. albipes and M. floricola are arboreal species. Both are supposed to feed less predators, as the physiology of arboreal ants is adapted to nitrogen-poor diets (Davidson and Patrell-Kim1996). The trophic signatures of both remained over 2.5 except for low shade, high forest distance agroforests. Arboreal ants typically exploit canopies and live on honeydew and few arthropods (Blüthgen et al. 2003). T. pasificum, C. reticulatus, and P. dives showed isotopic signatures lower $2.5 \delta^{15} \mathrm{~N}$. Such trophic positions are less than one distinct level (trophic level distance: $2.3-3.0 \delta^{15} \mathrm{~N}$ ) above the cacao leaf signature, pointing towards herbal nitrogen sources (Hood-Nowotny and Knols 2007; International Atomic Energy Agency 2009). Herbaceous diets of ants consist of liquids such as honeydew, plant sap or nectar. These resources are stationary, thus exploiting is less threatening than prey foraging (Davidson 1998). The carbon signatures of omnivorous ants have been species-specific. Such patterns cannot be explained without detailed behavioural observations. 
Possible explanations for the shown species-specific patterns of the analysed ants might be differences in foraging strategy and general behaviour. We have no behavioural observations. We now elucidate one possible explanation, which should be tested in future studies. A. gracilipes and O. simillimus are abundant and competitively strong omnivorous species. Both could have dominated carbohydrate resources as well as arthropod prey in cacao trees and displaced other ant species. Migration or resource adaptation could have been the behavioural responses of other ant species, to avoid superior competitors such as $A$. gracilipes and O. simillimus (Singer and Bernays 2003). Such a mechanism would explain trophic plasticity due to the local ant species assemblage, forest distance and shade cover. Displaced species would show isotopic signatures different from the predominant vegetation indicating food resource adaptation (Gratton and Forbes 2006; Girard et al. 2011a). Unfortunately, we do not possess isotope signatures of the whole plant community, or behavioural observations to identify particular food sources or behavioural adaptation.

\section{Conclusion}

In tropical agroforestry, trophic interactions are shaped by both local management and landscape context. Trophic levels occupied by an ant community can decrease with high levels of intensification (low shade cover and high distance to forests). Trophic positions of particular ant species show the same pattern (P. dives, $C$. reticulatus, $M$. floricola and T. albipes), while other species maintained stable tropic positions (A. gracilipes, T. pacificum, O. simillimus) and intraspecific trophic positions predicted the spatial ant species distribution. Thus, we infer both trophic plasticity and species turnover are the mechanisms defining the predatory function of ant communities. The relative importance of each mechanism might depend on the locally present ant species.

\section{Acknowledgements}

We are grateful to our Indonesian collaborates: CTFM office and lab at Tadulako University, Palu, administrative bodies and the Agricultural University Bogor (IPB). We particularly appreciate the help of Ilfianty Kasmundin and Pak Abdul Rauf. We thank Jens Dyckmans (KOSI, Göttingen) for great advice regarding isotope analyses and Nicola Holm for sample preparation. We are thankful to Akhmad Rizali (ants), Hardianto Mangopo (trees) and Firdaus (herbs) for sample identifications. We thank Arno Wielgoss and Iris Motzke for support during the whole project. We want to address special thanks to our head assistant Alfianus Rumede, lab assistants (Ivon, Abe and Fatma), field work assistants (Nimus, Idi, Tia), all 15 supportive cacao farmers, all ELUC-project partners, and every other cheerful helper. Finally, we acknowledge the financial support of the German Research Foundation (DFG) and the work of the coordinators of the DFG projects ELUC (CL-474/1-1) and EFForTS (CRC 990). 


\section{References}

Armbrecht I, Perfecto I, Silverman E (2006) Limitation of nesting resources for ants in Colombian forests and coffee plantations. Ecol Entomol 31:403-410. doi: 10.1111/j.13652311.2006.00802.x

Armbrecht I, Rivera L, Perfecto I (2005) Reduced Diversity and Complexity in the Leaf-Litter Ant Assemblage of Colombian Coffee Plantations. Conserv Biol 19:897-907.

Arnan X, Gaucherel C, Andersen AN (2011) Dominance and species co-occurrence in highly diverse ant communities: a test of the interstitial hypothesis and discovery of a three-tiered competition cascade. Oecologia 166:783-94. doi: 10.1007/s00442-011-1919-y

Bisseleua HBD, Fotio D, Yede, et al (2013) Shade tree diversity, cocoa pest damage, yield compensating inputs and farmers' net returns in West Africa. PLoS One 8:e56115. doi: 10.1371/journal.pone.0056115

Blüthgen N, Gebauer G, Fiedler K (2003) Disentangling a rainforest food web using stable isotopes: dietary diversity in a species-rich ant community. Oecologia 137:426-435. doi: 10.1007/s00442-003-1347-8

Bolton B (1994) Identification Guide to the Ant Genera of the World. doi: 10.1155/1994/62043

Bos MM, Steffan-Dewenter I, Tscharntke T (2007) Shade tree management affects fruit abortion, insect pests and pathogens of cacao. Agric Ecosyst Environ 120:201-205. doi: 10.1016/j.agee.2006.09.004

Bos MM, Tylianakis JM, Steffan-Dewenter I, Tscharntke T (2008) The invasive Yellow Crazy Ant and the decline of forest ant diversity in Indonesian cacao agroforests. Biol Invasions 10:1399-1409. doi: 10.1007/s10530-008-9215-4

Clough Y, Abrahamczyk S, Adams M-O, Anshary A, Ariyanti N, Betz L, Buchori D, Cicuzza D, Darras K, Putra DD, Fiala B, Gradstein SR, Kessler M, Klein A-M, Pitopang R, Sahari B, Scherber C, Schulze CH, Shahabuddin, Sporn S, Stenchly K, Tjitrosoedirdjo SS, Wanger TC, Weist M, Wielgoss A, Tscharntke T (2010) Biodiversity patterns and trophic interactions in human-dominated tropical landscapes in Sulawesi (Indonesia): plants, arthropods and vertebrates. In: Tscharntke T, Leuschner C, Veldkamp E, Faust H, Guhardja E, Bidin A (eds) (2010): Tropical Rainforests and Agroforests under Global Change. Ecological and Socioeconomic Valuations. Springer Verlag, Berlin, pp 15-71.

Dattilo W, Marquitti FMD, Guimarães PR, Izzo TJ (2014) The structure of ant-plant ecological networks: is abundance enough? Ecology 95:475-85.

Davidson DW (1998) Resource discovery versus resource domination in ants: a functional mechanism for breaking the trade-off. Ecol Entomol 23:484-490. doi: 10.1046/j.13652311.1998.00145.x

Davidson DW, Cook SC, Snelling RR, Chua TH (2003) Explaining the Abundance of Ants in Lowland Tropical Rainforest Canopies. Science (80- ) 300:969-972. doi: $10.1126 /$ science. 1082074 
Davidson DW, Patrell-Kim L (1996) Chapter 7 Tropical Arboreal Ants: Why So Abundant? In: Neotropical Biodiversity and Conservation. Gibson AC. University of California, Los Angeles, pp 127-140.

Dussutour A, Simpson SJ (2012) Ant workers die young and colonies collapse when fed a highprotein diet. Proc R Soc B Biol Sci 279:2402-2408. doi: 10.1098/rspb.2012.0051

Eubanks MD, Denno RF (1999) The ecological consequences of variation in plants and prey for an omnivorous insect. Ecology 80:1253-1266. doi: 10.1890/00129658(1999)080[1253:TECOVI]2.0.CO;2

Fagan WF (1997) Omnivory as a stabilizing feature of natural communities. Am Nat 150:554-567. doi: $10.1086 / 286081$

Fisher B (2010) Ants of Borneo (Ant course 2010 Guide to Genera).

Floren A, Biun A, Linsenmair E (2002) Arboreal ants as key predators in tropical lowland rainforest trees. Oecologia 131:137-144. doi: 10.1007/s00442-002-0874-z

Floren A, Wetzel W, Staab M (2014) The contribution of canopy species to overall ant diversity ( Hymenoptera : Formicidae ) in temperate and tropical ecosystems. Myrmecological News 6574.

Folgarait PJ (1998) Ant biodiversity and its relationship to ecosystem functioning: a review. Biodivers Conserv 7:1221-1244.

Fox J (2003) Effect Displays in $\{\mathrm{R}\}$ for Generalised Linear Models. J Stat Softw 8:1-27.

Fry B (2006) Stable Isotope Ecology. doi: 10.1007/0-387-33745-8

Girard J, Baril A, Mineau P, Fahrig L (2011) Carbon and Nitrogen Stable Isotope Ratios Differ among Invertebrates from Field Crops, Forage Crops, and Non-Cropped Land Uses. Ecoscience 18:98-109. doi: 10.2980/18-2-3390

Gonthier DJ, Ennis KK, Philpott SM, et al (2013) Ants defend coffee from berry borer colonization. BioControl 58:815-820. doi: 10.1007/s10526-013-9541-z

Gratton C, Forbes AE (2006) Changes in ??13C stable isotopes in multiple tissues of insect predators fed isotopically distinct prey. Oecologia 147:615-624. doi: 10.1007/s00442-005$0322-\mathrm{y}$

Grover CD, Kay AD, Monson JA, et al (2007) Linking nutrition and behavioural dominance: carbohydrate scarcity limits aggression and activity in Argentine ants. Proc Biol Sci 274:29512957. doi: $10.1098 /$ rspb.2007.1065

Haack KD, Vinson SB, Olson JK (1995) Food distribution and storage in colonies of Monomorium pharaonis (L.) (Hymenoptera: Formicidae). J Entomol Sci 30:70-81.

Hölldobler B, Wilson EO (1990) The Ants. Belknap Press of Harvard University Press, Massachusetts 
Hood-Nowotny R, Knols BGJ (2007) Stable isotope methods in biological and ecological studies of arthropods. Entomol Exp Appl 124:3-16. doi: 10.1111/j.1570-7458.2007.00572.x

International Atomic Energy Agency (2009) Manual for the Use of Stable Isotopes in Entomology. Vienna

Klein A-M, Steffan-Dewenter I, Tscharntke T (2006): Rain forest promotes trophic interactions and diversity of trap-nesting Hymenoptera in adjacent agroforestry. Journal of Animal Ecology 75: 315-323.

Kone M, Konate S, Yeo K, et al (2014) Effects of management intensity on ant diversity in cocoa plantation (Oume, centre west Côte d'Ivoire). J Insect Conserv 18:701-712. doi: $10.1007 / \mathrm{s} 10841-014-9679-8$

Kone M, Konate S, Yeo K, et al (2012) Changes in ant communities along an age gradient of cocoa cultivation in the Oumé region, central Côte d'Ivoire. Entomol Sci 15:324-339. doi: 10.1111/j.1479-8298.2012.00520.x

MacArthur RH, Wilson EO, MacArthur W (1967) The theory of island biogeography. Princet Univ Press Princet USA Major RE Kendal CE. doi: 10.2307/1796430

Madeira F, di Lascio A, Carlino P, et al (2014) Stable carbon and nitrogen isotope signatures to determine predator dispersal between alfalfa and maize. Biol Control 77:66-75. doi: 10.1016/j.biocontrol.2014.06.009

Meijer SS, Whittaker RJ, Borges P a. V. (2010) The effects of land-use change on arthropod richness and abundance on Santa Maria Island (Azores): unmanaged plantations favour endemic beetles. J Insect Conserv 15:505-522. doi: 10.1007/s10841-010-9330-2

Memmott J (1999) The structure of a plant-pollinator food web. Ecol Lett 2:276-280. doi: 10.1046/j.1461-0248.1999.00087.x

Mitchell MGE, Bennett EM, Gonzalez A (2014) Agricultural landscape structure affects arthropod diversity and arthropod-derived ecosystem services. Agric Ecosyst Environ 192:144-151. doi: 10.1016/j.agee.2014.04.015

Mollot G, Tixier P, Lescourret F, et al (2012) New primary resource increases predation on a pest in a banana agroecosystem. Agric For Entomol 14:317-323. doi: 10.1111/j.14619563.2012.00571.x

Naranjo SE, Gibson RL (1996) Phytophagy in predaceous Heteroptera: effects on life history and population dynamics. Zoophytophagous Heteroptera Implic. life Hist. Integr. pest Manag. pp $57-93$

Neves FS, Queiroz-Dantas KS, da Rocha WD, Delabie JHC (2013) Ants of three adjacent habitats of a transition region between the cerrado and caatinga biomes: the effects of heterogeneity and variation in canopy cover. Neotrop Entomol 42:258-68. doi: 10.1007/s13744-013-01237

Offenberg J, Thi Thu Cuc N, Wiwatwitaya D (2013) The effectiveness of weaver ant (Oecophylla smaragdina ) biocontrol in Southeast Asian citrus and mango. ASIAN MYRMECOLOGY 5:139-149. 
Oksanen J, FG B, Kindt, R, et al (2013) vegan: Community Ecology Package. 1-17.

Ottonetti L, Tucci L, Chelazzi G, Santini G (2008) Stable isotopes analysis to assess the trophic role of ants in a Mediterranean agroecosystem. Agric For Entomol 10:29-36.

Paine RT (1966) Food Web Complexity and Species Diversity. Am Nat 100:65. doi: $10.1086 / 282400$

Perfecto I, Vandermeer J (1996) Microclimatic changes and the indirect loss of ant diversity in a tropical agroecosystem. Oecologia 577-582.

Pfeiffer M, Mezger D, Dyckmans J (2013) Trophic ecology of tropical leaf litter ants ( Hymenoptera : Formicidae ) - a stable isotope study in four types of Bornean rain forest. 3141.

Philpott SM, Armbrecht I (2006) Biodiversity in tropical agroforests and the ecological role of ants and ant diversity in predatory function. Ecol Entomol 31:369-377. doi: 10.1111/j.13652311.2006.00793.x

Philpott SM, Foster PF (2005) Nest-Site Limitation in Coffee Agroecosystems: Artificial Nests Maintain Diversity of Arboreal Ants. Ecol Appl 15:1478-1485. doi: 10.1890/04-1496

Philpott SM, Perfecto I, Vandermeer J (2008) Effects of predatory ants on lower trophic levels across a gradient of coffee management complexity. J Anim Ecol 77:505-511. doi: 10.1111/j.1365-2656.2008.01358.x

Philpott SM, Perfecto I, Vandermeer J (2006) Effects of Management Intensity and Season on Arboreal Ant Diversity and Abundance in Coffee Agroecosystems. Biodivers Conserv 15:139-155. doi: 10.1007/s10531-004-4247-2

Pinheiro J, Bates D (2014) Linear and Nonlinear Mixed Effects Models.

Pocock MJO, Evans DM, Memmott J (2012) The robustness and restoration of a network of ecological networks. Science 335:973-7. doi: 10.1126/science.1214915

Ponsard S, Arditi R (2000) What Can Stable Isotopes ( $\ 15 \mathrm{~N}$ and $\ 13 \mathrm{C}$ ) Tell About the Food Web of Soil Macro-Invertebrates? Ecology 81:852-864. doi: 10.1890/00129658(2000)081[0852:WCSINA]2.0.CO;2

R Core Team (2014) R: A language and environment for statistical computing. R Found Stat Comput Vienna, Austria URL http://www.R-project.org/.

Rice RA, Greenberg R (2000a) Cacao Cultivation Biological Diversity and the Conservation of Biological Diversity. Ambio 29:167-173. doi: 10.1579/0044-7447-29.3.167

Rice RA, Greenberg R (2000b) Cacao Cultivation and the Conservation of Biological Diversity. AMBIO A J Hum Environ 29:167-173. doi: 10.1579/0044-7447-29.3.167

Rizali A, Clough Y, Buchori D, et al (2013) Long-term change of ant community structure in cacao agroforestry landscapes in Indonesia. Insect Conserv Divers 6:328-338. doi: 10.1111/j.17524598.2012.00219.x 
Rizali A, Lohman DJ, Buchori D, et al (2010) Ant communities on small tropical islands: effects of island size and isolation are obscured by habitat disturbance and "tramp" ant species. J Biogeogr 37:229-236. doi: 10.1111/j.1365-2699.2009.02194.x

Rosenheim JA (2001) Source-sink dynamics for a generalist insect predator in habitats with strong higher-order predation. Ecol Monogr 71:93-116. doi: 10.1890/00129615(2001)071[0093:SSDFAG]2.0.CO;2

Rudolph KP, Palmer TM (2013) Carbohydrate as Fuel for Foraging, Resource Defense and Colony Growth - a Long-term Experiment with the Plant-ant Crematogaster nigriceps. Biotropica 45:620-627. doi: 10.1111/btp.12040

Singer MS, Bernays EA (2003) Understanding Omnivory Needs a Behavioral Perspective. Ecology 84:2532-2537.

Thompson RM, Hemberg M, Starzomski BM, Shurin JB (2007) Trophic Levels and Trophic Tangles: The Prevalence of Omnivory in Real Food Webs. Ecology 88:612-617.

Tillberg CV, McCarthy DP, Dolezal AG, Suarez AV (2006) Measuring the trophic ecology of ants using stable isotopes. Insect. Soc. 53:65-69, doi: 10.1007/s00040-005-0836-7

Van Walsum E, Gobin B, Ito F, Billen J (1998) Worker reproduction in the ponerine ant Odontomachus simillimus (Hymenoptera: Formicidae). Sociobiology 32:427-440.

Vandermeer J, Perfecto I, Nuñez GI, et al (2002) Ants (Azteca sp.) as potential biological control agents in shade coffee production in Chiapas, Mexico. Ecology 56:271-276. doi: 10.1023/A:1021328820123

Venables WN, Ripley BD (2002) Modern Applied Statistics with S Fourth edition by. World. doi: $10.2307 / 2685660$

Vinson SB (1968) The distribution of an oil, carbohydrate, and protein food source to members of the imported fire ant colony. J Econ Entomol 61:712-714.

Way MJ, Heong KL (2009) Significance of the tropical fire ant Solenopsis geminata (hymenoptera: formicidae) as part of the natural enemy complex responsible for successful biological control of many tropical irrigated rice pests. Bull Entomol Res 99:503-512. doi: $10.1017 /$ S0007485308006524

Wielgoss A, Tscharntke T, Buchori D, et al (2010) Temperature and a dominant dolichoderine ant species affect ant diversity in Indonesian cacao plantations. Agric Ecosyst Environ 135:253259. doi: 10.1016/j.agee.2009.10.003

Wielgoss A, Tscharntke T, Rumede A, et al (2014) Interaction complexity matters: disentangling services and disservices of ant communities driving yield in tropical agroecosystems. Proc R Soc B 281:20132144. doi: http://dx.doi.org/10.1098/rspb.2013.2144

Wood S (2000) Modelling and smoothing parameter estimation with multiple quadratic penalties. J R Stat Soc Ser B (Statistical Methodol 62:413-428. 
Woodcock P, Edwards DP, Newton RJ, et al (2013) Impacts of intensive logging on the trophic organisation of ant communities in a biodiversity hotspot. PLoS One 8:e60756. doi: 10.1371/journal.pone.0060756

Woodcock P, Edwards DP, Newton RJ, et al (2012) Assessing trophic position from nitrogen isotope ratios: effective calibration against spatially varying baselines. Naturwissenschaften 99:275-283. doi: 10.1007/s00114-012-0896-2

\section{Supporting information}


Supporting information - Chapter 4

$140 / 168$ 


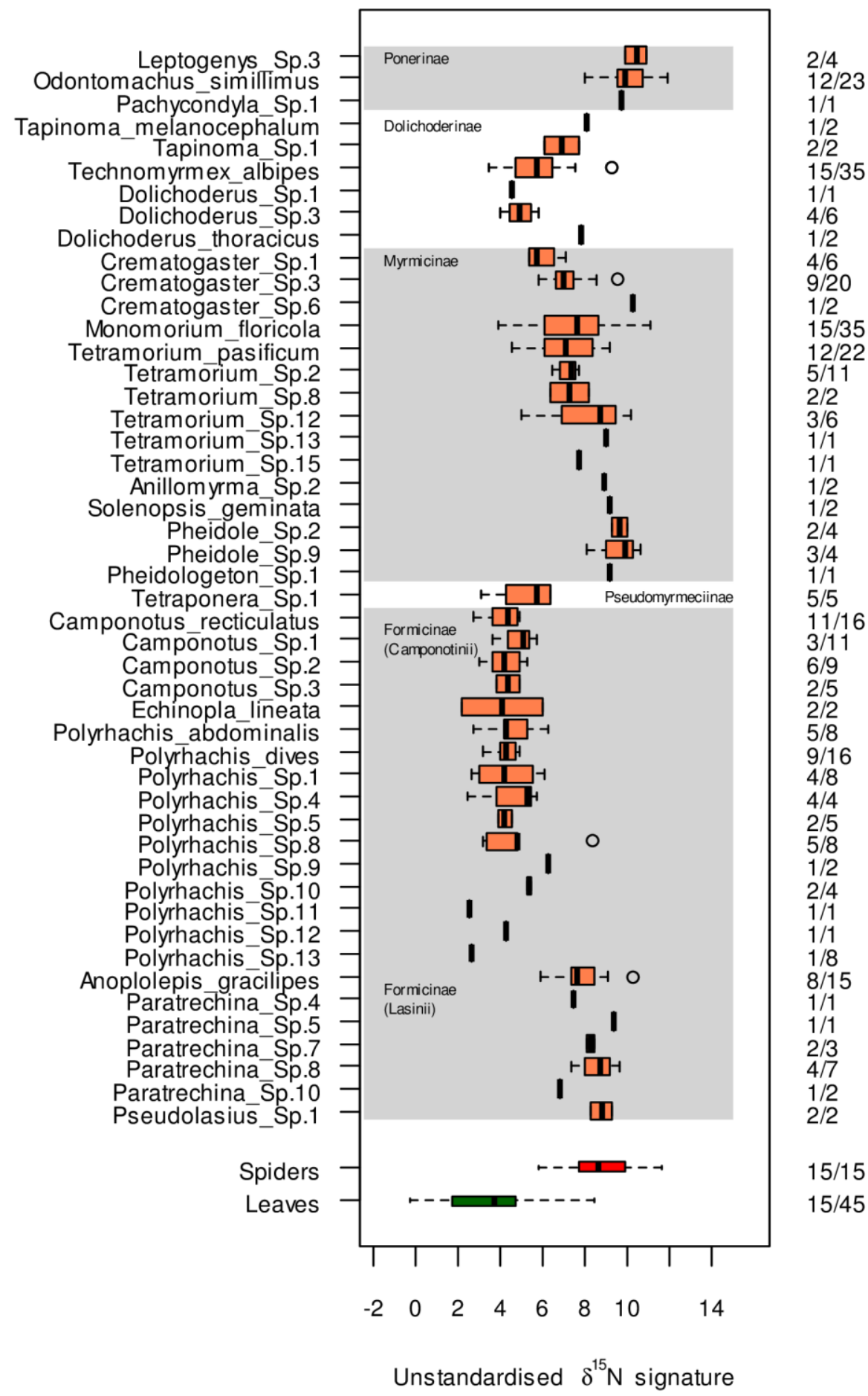

Fig. S1. Unstandardized $\delta 15 \mathrm{~N}$ values of all ant (morpho-) species.

Variation in delta $\mathrm{N}$ between different ant species collected from cacao plantations in Sulawesi, Indonesia. Values for cacao leaves and spiders added for comparison. Ants are presented ordered by family (and tribe in the case of Formicidae). The right axis shows number of samples analysed and number of plantations covered by the samples. 


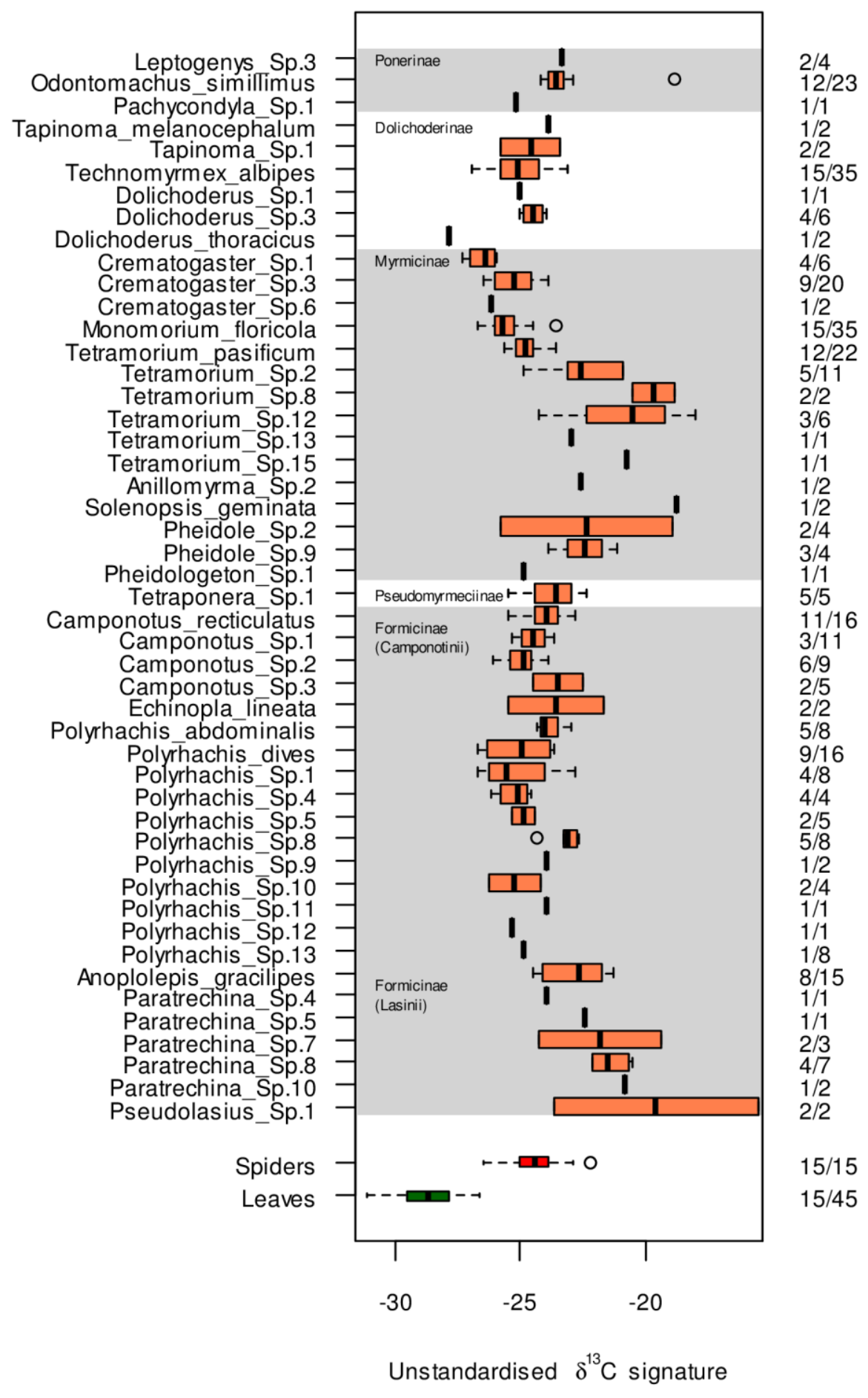

Fig. S2. Unstandardized $\delta 13 \mathrm{C}$ values of all ant (morpho-) species.

Variation in delta $\mathrm{C}$ between different ant species collected from cacao plantations in Sulawesi, Indonesia. Values for cacao leaves and spiders added for comparison. Ants are presented ordered by family (and tribe in the case of Formicidae). The right axis shows number of samples analysed and number of plantations covered by the samples. 


\section{3 / 168}


144 / 168 


\section{Chapter 5: Intra-specific plasticity in dietary resource-use maintains inter-specific complementarity in natural ant communities}

Yann Clough, Pierre Gras, Akhmad Rizali, Teja Tscharntke

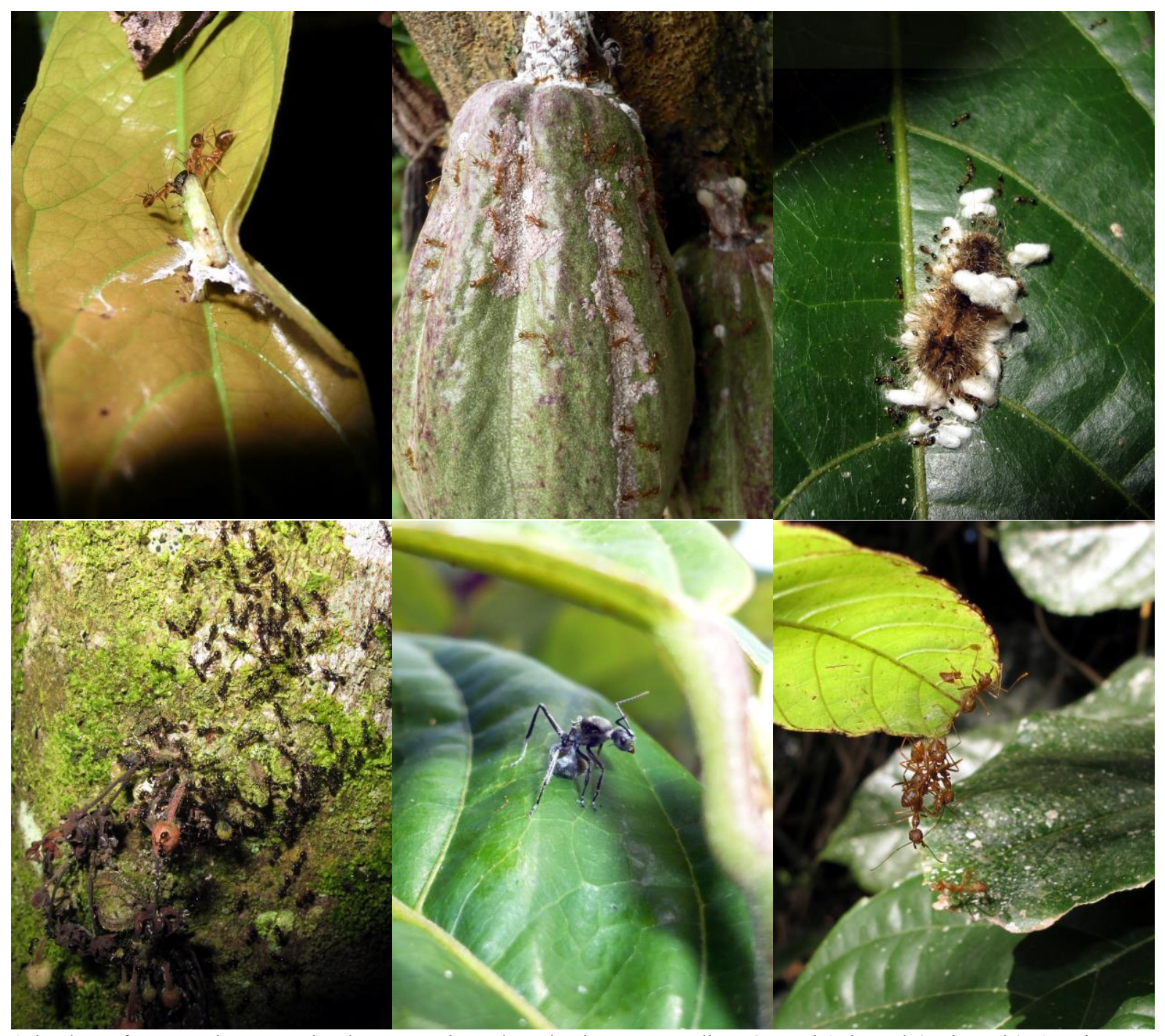

Selection of ant species occurring in cacao plantations in the Napu Valley, Central Sulawesi (Indonesia). Starting topleft: (1) Anoplolepis gracilipes (SMITH, 1857) feeding on caterpillar, (2) A. gracilipes with trophobionts (mealybugs), (3) Monomorium floricola (JERDON, 1851) scavenging on parasitized caterpillar, (4) Myrmicinae sp. (LEPELETIER DE SAINTFARGEAU, 1835) with aphids at cacao flowers (tree trunk), (5) Polyrbacbis cf. dives (SMITH, 1857) on a leaf, and (6) Oecophylla smaragdina (FABRICIUS, 1775) creating ant bridge. 
$146 / 168$ 


\section{Summary}

Organisms may adjust their diet when ecologically similar species are present, resulting in increased complementarity and opportunity for coexistence. Evidence from unmanipulated, species-rich animal communities however is scarce. Ants are an omnivorous group ranging from predominantly herbivorous to predominantly predatory species. They exhibit various strategies by which interspecific interference is reduced, including spatial and temporal partitioning of resources. Here, we test whether ant communities show evidence of systematic trophic shifts resulting in increased complementarity. We use nitrogen and carbon stable isotope data from a multi-species, multi-site study as a surrogate for ant trophic level and basal food source, respectively. Using a randomization test, we show that observed nitrogen and carbon isotopic signatures of ants sampled within a community are significantly more different from each other than would be expected by chance. Evidence suggests these shifts are not restricted to feeding on different food sources within a trophic level but can result in a change in the mixture among food sources of different trophic levels. These results demonstrate that ant species in a community will exploit resources in a complementary way, most likely to minimize costs related to interspecific interference. The enhanced complementarity due to trophic shifts could mean that ecosystem functioning may be more stable than expected across heterogeneous, highly diverse communities.

\section{Introduction}

Explaining the coexistence of multiple, ecologically similar species at small spatial scales is a classical question in ecology (Hutchinson 1957). Ecological theory suggests that ecologically very similar species should not be able to coexist in a stable manner, as they would compete for resources until one of the species goes extinct (Gause 1932). A widely recognized mechanism that enables diversity within local communities is the partitioning of resource-use (or niches) occurring when similar species use different parts of their habitats, forage at different times, or take different food items (Schoener 1974). These are often interpreted as being the result of evolutionary or behavioural shifts undertaken to reduce interspecific competition and associated fitness costs (Schoener 1974). While it is heavily debated to what extent competition shapes coevolution of species (Connell 1980), examples of niche shifts in ecological time, e.g. in the form of shifts in resource-use in a species when another species is introduced, abound (Inouye 1978, Werner and Hall 2007, Bolnick et al. 2010, Fründ et al. 2013, Zuppinger-Dingley et al. 2014).

Possibly more than any other group, ant communities are widely seen as being driven by interspecific competition (the "hallmark of ant ecology"; (Hölldobler and Wilson 1990)). Spatial mosaics of non-co-occurring ant species, and the large impacts of invasive ants on native ant 
communities are some of the patterns cited as evidence. However, ant communities can be extremely species-rich locally, not only in structurally complex habitats, but even in fairly homogenous habitats (Feener et al. 2008), suggesting that the impacts of competition are substantially weakened by other processes (Cerdá et al. 2013). Trivially, many ant species do not compete because they use complementary food resources: ant communities include predators and scavengers as well as nectar- and honeydew feeders (Davidson et al. 2003, Blüthgen et al. 2003). Many species have mixed diets, however, and when preferred range of resources overlap between ant species, one might expect that workers of different species change their foraging, trading-off resource quality against avoiding inter-specific interference. This is supported by experiments showing that ant workers forage on a wider diversity of food sources in a multi-species than in a single-species context (Blüthgen and Fiedler 2004). Whether these responses to artificial food sources and community manipulations also apply to longer term resource-use in unmanipulated, natural ant communities is unknown.

Here we test whether the resource-uses of ants within species suggest systematic shifts in food resources in response to the presence of trophically similar species. The data originate from 15 cacao plantations in Sulawesi, Indonesia, where ant communities are diverse and functionally important (Bos et al. 2007, Wielgoss et al. 2010, 2014). Using the nitrogen and carbon stable isotopic ratios $\left(\delta^{15} \mathrm{~N}, \delta^{13} \mathrm{C}\right)$ as indicators for trophic position and basal food source, respectively, we test whether the pair-wise distances between values ants take along these two axes are larger within a community than would be expected given the isotopic ratios the same species take across communities. Stable isotopes are increasingly being used in ant ecology to show how level of community- or species shifts in trophic level in response to changes in land-use (Gibb and Cunningham 2011, Woodcock et al. 2013), and are well suited to test for long-term changes in diet as the isotopic ratios integrate the diet signal over a long period of time. Using both carbon and nitrogen isotopes allows to see if shifts are likely to occur within a trophic level (shift in $\delta^{13} \mathrm{C}$ but not $\delta^{15} \mathrm{~N}$ ) or between trophic levels (shift in $\delta^{15} \mathrm{~N}$ ). Finally, we test which species contribute to these shifts.

\section{Material and methods}

\section{Study sites}

In early June 2012, we sampled ants within smallholder cacao plantations at the eastern border of the Lore Lindu National Park in Central Sulawesi, Indonesia ( $1^{\circ} 25^{\prime} 31.8^{\prime \prime} \mathrm{S}, 120^{\circ} 18^{\prime}$ 57.55" E). The experimental plantations were located close to the villages Watumaeta, Wuasa and Banyusari in the northern part of the Napu valley. This part of the valley is situated at $\sim 1130 \mathrm{~m}$ 
a.s.l. Precipitation varied from $1990 \mathrm{~mm}$ to $3804 \mathrm{~mm}$ between plantations. Each plantation covered between 0.5 and 1.5 ha. Plantations were separated by minimal distance of $500 \mathrm{~m}$ (edgeto-edge). Local farmers managed all plantations, but we implemented spacious insecticide, fungicide and herbicide application free areas covering the sampling area and at least 2 corridors to the plantation border in each plantation. This area had not been sprayed with pesticides for at least 14 months. The non-crop plant community mainly consisted of two planted legume shade tree species (Erythrina subumbrans MERR and Gliricidia sepium KUNTH). Both tree species represented 30 - $91 \%$ of non-crop trees and $61 \%$ of all trees within the chemical application free area of all plantations (see chapter 2, Table S1). We trimmed the herb layers every two months with a motor scythe. The most abundant herbal plants (see chapter 2, Table S2) were Ageratum conyzoides L. (Asteraceae), Bidens pilosa L. (Asteraceae), Eleusine indica (L.) GAERTN. (Poaceae), and Borreria laevis GRISEB. (Rubicaceae). The ant communities differed between the 15 plantations: Shannon index: 0.31 - 1.77, (morpho-) species richness: 10 - 18, (morpho-) species evenness: 0.13 - 0.69; and number of recorded individuals per plantation: 569 - 4985. In total, we recorded 52 ant (morpho-) species (see chapter 2, Tables S3, 4).

\section{Sampling and analysing methods}

Ant and plant sampling. We sampled ants within cacao plantations organised in plots covering 25 cacao trees in a five-times-five grids, each covering approximately $225 \mathrm{~m}^{2}$ (three metre intertree distance). During 10 days, we collected all samples of animal and plant tissue within all plantations. To handle samples not powdered latex gloves were obligatory. All collected arthropod and plant samples were immediately stored in a freezer $\left(-18^{\circ} \mathrm{C},<5 \mathrm{~h}\right.$ after sampling in the field). When the survey was done, we carried the frozen samples to UNTAD University (Palu, Central Sulawesi), where we kept the samples frozen until drying in a ventilated drying cabinet. All dry samples were stored in Eppendorf tubes, in plastic bags embedded in silica gel in hermetic proofed plastic boxes. In December 2012 the samples arrived at the Georg-August University Göttingen (Germany). The silica gel - despite still dry - was immediately replaced. During further preparation, we kept the samples in a dark, dry environment with a stable temperature of $16-18^{\circ} \mathrm{C}$.

Stable isotope analyses. We used only the head of an ant individual for stable isotope analyses. We milled the leave tissue using an electronic mill (Janke \& Kunkel, tabletop hammer mill type: MFC, $5000 \mathrm{r} / \mathrm{min}$ ), and pestled the spider samples. Depending on the suspected nitrogen content, we measured 0.5 to $1 \mathrm{mg}$ of animal tissue, and 1 - $2 \mathrm{mg}$ of plant tissue using a micro scale (Sartorius micro, $0.001-3.000 \mathrm{mg}, \mathrm{d}<0.001 \mathrm{mg})$ to fill in tin capsules $(5 \mathrm{x} 8 \mathrm{~mm})$. Stable isotope measurements were conducted by isotope mass spectrometry (Delta+, Termo Finnigan) coupled to an elemental 
analyser (NA1110, CEE - Instruments). The stable isotope analyses were conducted at the Centre for Stable Isotope Research and Analysis, University of Göttingen.

\section{Statistical analyses}

Site-level variation in $\delta^{15} \mathrm{~N}$ and $\delta^{13} \mathrm{C}$ values across trophic levels is expected given variation in precipitation, agricultural management (e.g. fertilization), soils, species community composition of autotrophs as well as consumers preyed upon by ants. Since our focus is on the relative position of ants compared to other ants within the same site, we standardize the values for each site, by subtracting the mean and dividing by the standard deviation of $\delta$. We then test whether the difference between standardised $\delta$ values of species within plots is greater than would be expected by chance. To do that, we first generate 10000 randomized sets of species communities identical in species composition to the observed communities, but for which the standardised $\delta$ values for each species are sampled without replacement from the observed vector of standardised $\delta$ value of the same species. In other words, standardised $\delta$ values are reshuffled within species but between communities. This procedure is applied jointly to the standardised $\delta^{15} \mathrm{~N}$ and $\delta^{13} \mathrm{C}$ values.

For the observed and the randomized communities, we calculate the sum of squared differences between relative $\delta$ values. P-values are then simply derived from the number of values divided by two (to obtain two-sided P-values).

For each species, we calculate an index of contribution to increases in complementarity, by recalculating the $\mathrm{P}$-values above leaving the species out, and dividing that $\mathrm{P}$-value by the P-value for the complete community. A large ratio suggests the species contributes a lot to the observed, community wide pattern.

\section{Results}

We found and sampled workers from a mean of 12 (morpho-) species per site (range: 9 18), and total of 48 species. The list of species and abundances at baits can be found in the supplementary material (see Chapter 2, Tables S3,4). The $\delta^{15} \mathrm{~N}$ values of the ants differ systematically between species and subfamilies (Figure S1). 

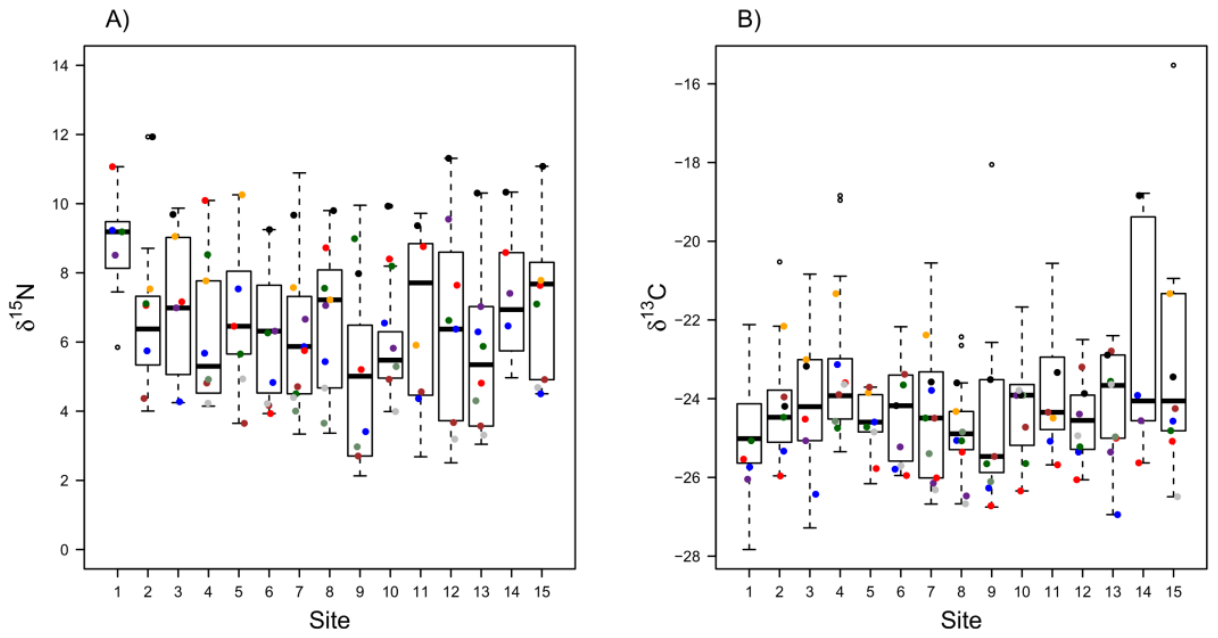

- Technomyrmex albipes

- Crematogaster sp.3

Monomorium floricola

- Tetramorium pacificum

- Camponotus reticulatus

- Camponotus sp.2

Odontomachus simillimus

Polyrhachis dives

Fig. 1. (A) $\delta^{15} \mathrm{~N}$ and (B) $\delta^{13} \mathrm{C}$ values for ants in smallholder cacao sites in Indonesia. Points indicate the species- and site specific values for species that occurred in most of the sites, while boxplots summarize distribution of values over whole communities in a site (bold lines are medians, hinges indicate first and second quartiles, whiskers extend to the most extreme data point which is no more than 1.5 times the length of the box away from the box, with more extreme points plotted as open circles).

Mean and variance in distribution of stable isotope ratios varied between sites, but are approximately normally distributed within sites (Fig. 1A and B).

A)

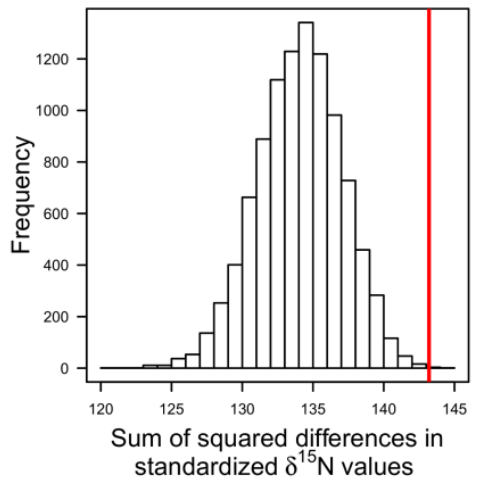

B)

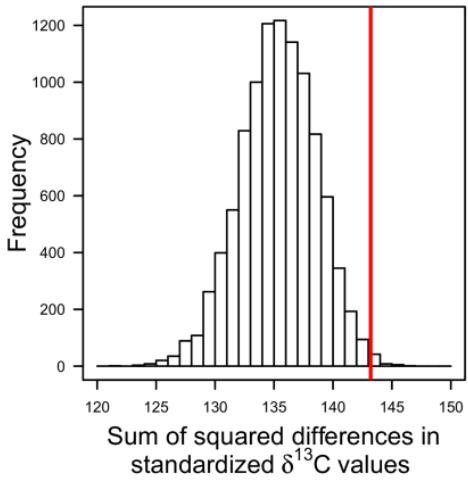

C)

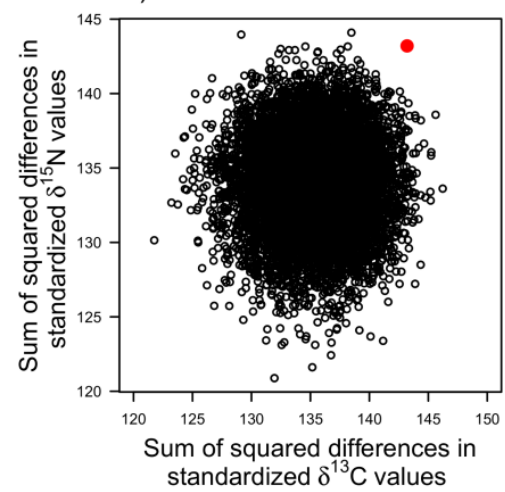

Fig. 2. Sum of the squared differences between $(\mathrm{A}, \mathrm{C})$ relative $\delta^{15} \mathrm{~N}$ and $(\mathrm{B}, \mathrm{C}) \delta^{13} \mathrm{C}$ values within ant communities, based on 1000 randomizations (histogram) and the observed data (single values indicated by a bold red vertical line in (a) and (b), and an oversized point in (c)).

The randomization tests show that the observed difference between relative $\delta^{15} \mathrm{~N}$ values of ants within communities is significantly larger than expected by chance $(\mathrm{P}=0.00025)$. The randomization test for $\delta^{13} \mathrm{C}$ values is also significant, but not as strongly so $(\mathrm{P}=0.00265)$. 


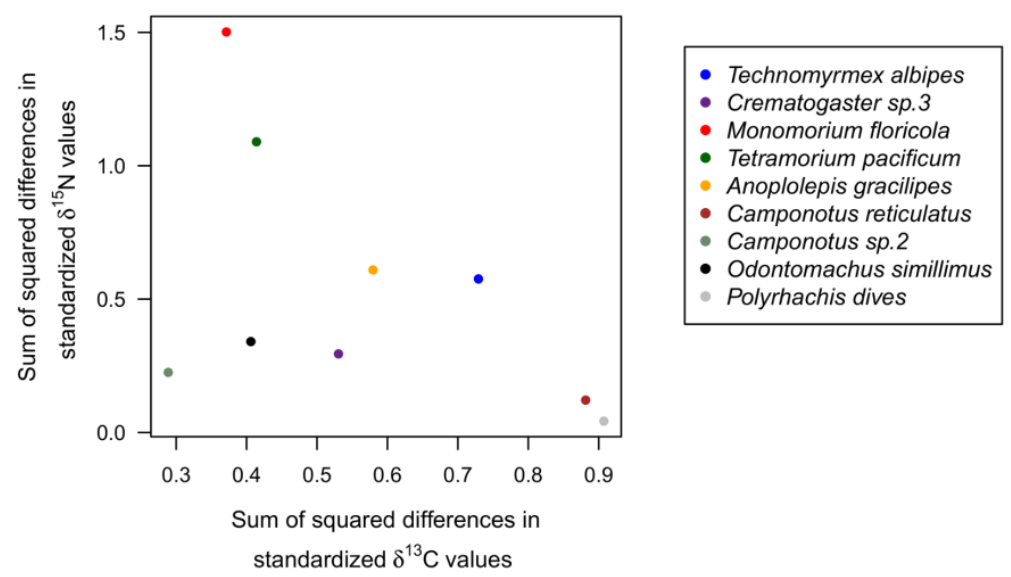

Fig. 3. Sum of the squared intraspecific differences between relative $\delta^{15} \mathrm{~N}$ and $\delta^{13} \mathrm{C}$ values

Among those species occurring in more than five sites, there was strong variability in the contribution of nitrogen and carbon isotopic ratios in overall variability (Fig. 1, 3).

Table 1. Species-level contribution to the complementarity in resource-use

\begin{tabular}{lll}
\hline species & \multicolumn{2}{c}{ Ratios $\left(\mathrm{P}_{\text {leave-one-out }} / \mathrm{P}_{\text {complete community }}\right.$ ) } \\
Citrogen & 1.6 \\
\hline Anoplolepis gracilipes & 1.2 & 0.8 \\
Camponotus reticulatus & 0.8 & 1.0 \\
Camponotus sp.2 & 0.8 & 3.0 \\
Crematogaster sp.3 & 1 & 0.8 \\
Monomorium floricola & 10.6 & 1.6 \\
Odontomachus simillimus & 0.4 & 2.1 \\
Polyrhachis dives & 1.2 & 0.4 \\
Technomyrmex albipes & 1.2 & 1.6 \\
Tetramorium pacificum & 8.4 &
\end{tabular}

A strong variation along the $\delta^{15} \mathrm{~N}$ axis, but little variation along the $\delta^{13} \mathrm{C}$ axis was found in Monomorium floricola (JERDON, 1851) and Tetramorium pacificum (MAYR, 1870). Camponotus reticulatus (Roger, 1863), and Polyrbachis dives (SMITH, 1857) were highly variable on $\delta^{13} \mathrm{C}$ axis but least variable along the $\delta^{15} \mathrm{~N}$ axis. Technomyrmex albipes (SMITH, 1861) and Anoplolepis gracilipes (SMITH, 1857) show intermediate variability along both axes, while Camponotus sp.2 (Genus: MAYR, 1861), Odontomachus simillimus (SMITH, 1858), and Crematogaster sp.3 (Genus: LuND, 1831) had low to medium variability along both axes. Contribution to the complementarity along $\delta^{15} \mathrm{~N}$ axis ranged from a P-value ratio from 0.4 for $O$. simillimus to 10.6 for $M$. floricola, while contribution to the complementarity along the $\delta^{13} \mathrm{C}$ axis ranged from a P-value from 0.4 for T. albipes to 3.0 for Crematogaster sp.3 (Table 1). 


\section{Discussion}

Our results demonstrate that ants shift their food resource-use to be more different from that of co-occurring species, that these shifts are consistent enough across time to be detected in adult ant worker tissue, and that they most likely involve changes in food items across as well as within trophic levels. While community-wide as well as intra-specific, cross-habitat variability in food resource-use by ants have previously been reported (Gibb and Cunningham 2011, Woodcock et al 2013), we show using a null model approach that the relative values of isotope ratios of ant worker tissues within a community are more different across species than one would expect based on the distribution of values within species. It is not the intraspecific variability itself which is remarkable, but the consistency with which this variability increases complementarity in resourceuse. These findings complement experiments which showed that the choice of food items by ants is conditional on the presence or absence of foraging workers of other ant species (Blüthgen and Fiedler 2004). Our study shows for naturally occurring communities that these behavioural responses are consistent enough to be detected in isotopic ratios over time despite the variability in resource-use within ant colonies over time (see chapter 3).

Competition and resource partitioning have been particularly intensively studied in ant communities, but we would expect similar findings for other communities of generalistic consumers. For instance, shifts in resource-use in response to a change in number or identity of co-occurring species have been reported for pollinator communities foraging on flowers in a variety of experimental and non-experimental settings (Inouye 1978, Fontaine et al. 2008, Fründ et al. 2013).

Encountering workers of other species at a food source can be costly in terms of time and metabolic resources, and may cause mortality due to predation. Many species monopolize food resources and keep workers of other species from accessing them, forcing them to opt for alternative resources. Our results suggest that such shifts are common, but differ between species in intensity and trophic nature. Numerically dominant species such as M. floricola and T. pacificum, and to a lesser degree $A$. gracilipes and T. albipes shift resources across trophic levels. These species are trophobiont-tending but also predate and scavenge. Nectar-feeding and trophobiont tending Camponotini have little opportunity for variation across the $\delta^{15} \mathrm{~N}$ axis, but some species, such as C. reticulatus and $P$. dives, can vary strongly across the $\delta^{13} \mathrm{C}$ axis. The "leave-one-out" approach allowed us to disentangle variability from contribution to complementarity. Interestingly, some species that vary a lot in relative trophic role across ant community contribute much to complementarity, while others do not. M. floricola and T. pacificum, two species occupying very different position along the $\delta^{15} \mathrm{~N}$ axis in different communities, were both involved maintaining complementarity, either by shifting or inducing other species to shift. While A. gracilipes and T. 
albipes were more variable than O. simillimus or Crematogaster sp. 3, they did not contribute more to complementarity than these two species. Similarly, along the $\delta^{15} \mathrm{C}$ axis, $P$. dives and $C$. reticulatus were both vary variable, but only the former contributed to complementarity. This shows that while increasing complementarity to the diet of other ant species is a mechanism driving intraspecific variability for some species, this seems to be less important for other species.

While our study addressed only trophic signal, partitioning of food items is only one of several approaches ants can use to reduce interference competition. There are of course many other differences in resource-use among the species in the communities we studied, which may further reduce the niche overlap. Several of the Camponotus species forage mainly at night, whereas other species, especially numerically dominant dolichoderines and myrmicines, tend to be most active during the day. Whilst some of the species are entirely arboreal, others, such as $A$. gracilipes and Odontomachus spp. nest and forage at least partly on the ground. Multi-site, multi-species characterisation of multiple ecological and behavioural traits would allow to move beyond the findings of this study, and to evaluate the relative role of trophic shifts in niche partitioning. It has recently been highlighted that ant community studies addressing competition and resource partitioning may identify different drivers depending on the context (Cerdá et al. 2014). Whilst it is tempting to generalize our findings to ant communities in other systems, relying on the plausibility of that omnivorous consumer more easily shift food resources than other dimensions of the niche, this could be misleading. Habitat complexity strongly affects ant communities, possibly through the associated diversity of niches (Lassau and Hochuli 2004, Klimes et al. 2012). The tree crop systems in which we conducted our study are more simply structured than rainforests, for example, and evenly shaded, thus reducing the opportunity of shifts along microhabitat and microclimatic gradients.

By demonstrating diet shifts conditional on co-occurring species, our results suggest a promising approach to understand competition among ants is to quantify addressing fitness constraints these shifts may be imposing on ant colonies. It would also be interesting to analyse whether the absence of species in some communities could be associated with the inability to shift diets, which would allow us to better understand ant community assembly.

Ants are functionally important in many ecosystems, and in our study system, cacao yield depends on the ecology of the dominant ant (Wielgoss et al. 2014, and see chapter 2). Our results suggest that intra-specific variability in resource-use could make predicting ecological interactions, functions and processes in communities from species-level ("mean") traits more challenging. On the other hand, the enhanced complementarity due to trophic shifts could mean that certain functions and processes may be more stable than expected across heterogeneous, highly diverse communities. 


\section{Acknowledgments}

We are grateful to our Indonesian cooperation collaborates of the CTFM office and lab at the Tadulako University, Palu. Moreover, we acknowledge the support of all administrative bodies and of the Agricultural University Bogor (IPB) that helped us to perform this study. We particularly appreciate the administrative help of Ilfianty Kasmundin and Pak Abdul Rauf. We thank Jens Dyckmans (KOSI, Göttingen) for great advice regarding Isotope based analyses. We are thankful to Akhmad Rizali for ant identifications, and Hardianto Mangopo (trees) as well as Firdaus (herbs) for plant identifications. We thank Arno Wielgoss and Iris Motzke for guidance in the study region and great support during the whole project. We would like to address special thanks to our head assistant Alfianus Rumede, lab assistants (Ivon, Abe and Fatma), and field work assistants (Nimus, Idi, Tia), all 15 supportive cacao farmers, and all members of the ELUC-project, as well as every other cheerful helper. We appreciate the work of the German coordination teams of the DFG projects ELUC (CL-474/1-1) and Collaborative research centre 990 (EFForTS), especially Wolfram Lorenz. Finally, we acknowledge the financial support of the German Research Foundation DFG (Environmental land-use change project ELUC, CL-474/1-1) and through the CRC 990 (Ecological and Socioeconomic Functions of Tropical Lowland Rainforest Transformation Systems in Sumatra, Indonesia, EFForTS).

\section{References}

Blüthgen, N., and K. Fiedler. 2004. Competition for composition: lessons from nectar-feeding ant communities. Ecology 85:1479-1485.

Blüthgen, N., G. Gebauer, and K. Fiedler. 2003. Disentangling a rainforest food web using stable isotopes: dietary diversity in a species-rich ant community. Oecologia 137:426-435.

Bolnick, D. I., T. Ingram, W. E. Stutz, L. K. Snowberg, O. L. Lau, and J. S. Paull. 2010. Ecological release from interspecific competition leads to decoupled changes in population and individual niche width. Proceedings of the Royal Society B: Biological Sciences 277:1789-1797.

Bos, M. M., I. Steffan-Dewenter, and T. Tscharntke. 2007. The contribution of cacao agroforests to the conservation of lower canopy ant and beetle diversity in Indonesia. Biodiversity and Conservation 16:2429-2444.

Cerdá, X., X. Arnan, and J. Retana. 2013. Is competition a significant hallmark of ant (Hymenoptera: Formicidae) ecology? Myrmecological News 18:131-147.

Connell, J. H. 1980. Diversity and the coevolution of competitors, or the ghost of competition Past. Oikos 35:131-138.

Davidson, D. W., S. C. Cook, R. R. Snelling, and T. H. Chua. 2003. Explaining the abundance of ants in lowland tropical rainforest canopies. Science 300:969-72. 
Feener, D. H., M. R. Orr, K. M. Wackford, J. M. Longo, W. W. Benson, and L. E. Gilbert. 2008. Geographic variation in resource dominance-discovery in Brazilian ant communities. Ecology 89:1824-1836.

Fründ, J., C. F. Dormann, A. Holzschuh, and T. Tscharntke. 2013. Bee diversity effects on pollination depend on functional complementarity and niche shifts. Ecology 94:2042-2054.

Gause, G. 1932. Experimental studies on the struggle for existence I. Mixed population of two species of yeast. Journal of Experimental Biology 9:389-402.

Gibb, H., and S. A. Cunningham. 2011. Habitat contrasts reveal a shift in the trophic position of ant assemblages. Journal of Animal Ecology 80:119-127.

Hölldobler, B., and E. O. Wilson. 1990. The Ants. Page 746. Belknap Press of Harvard University Press, Massachusetts.

Hutchinson, G. E. 1957. Concluding remarks. Pages 415-427 Cold Spring Harbor Symposia on Quantitative Biology (Vol. 22). Cold Spring Harbor Laboratory Press.

Inouye, D. W. 1978. Resource partitioning in bumblebees: experimental studies of foraging behavior. Ecology 59:672-678.

Klimes, P., C. Idigel, M. Rimandai, T. M. Fayle, M. Janda, G. D. Weiblen, and V. Novotny. 2012. Why are there more arboreal ant species in primary than in secondary tropical forests? Journal of Animal Ecology 81:1103-1112.

Lassau, S. A., and D. F. Hochuli. 2004. Effects of habitat complexity on ant assemblages. Ecography 27:157164.

Schoener, T. W. 1974. Resource partitioning in ecological communities. Science 185:27-39.

Werner, E. E., and D. J. Hall. 2007. Foraging efficiency and habitat switching in competiting sunfishes. Ecology 60:256-264.

Wielgoss, A., T. Tscharntke, D. Buchori, B. Fiala, and Y. Clough. 2010. Temperature and a dominant dolichoderine ant species affect ant diversity in Indonesian cacao plantations. Agriculture, Ecosystems and Environment 135:253-259.

Wielgoss, A., T. Tscharntke, A. Rumede, B. Fiala, H. Seidel, S. Shahabuddin, and Y. Clough. 2014. Interaction complexity matters: disentangling services and disservices of ant communities driving yield in tropical agroecosystems. Proceedings. Biological sciences / The Royal Society 281:20132144.

Woodcock, P., D. P. Edwards, R. J. Newton, C. Vun Khen, S. H. Bottrell, and K. C. Hamer. 2013. Impacts of intensive logging on the trophic organisation of ant communities in a biodiversity hotspot. PLoS ONE 8.

Zuppinger-Dingley, D., B. Schmid, J. S. Petermann, V. Yadav, G. B. De Deyn, and D. F. B. Flynn. 2014. Selection for niche differentiation in plant communities increases biodiversity effects. Nature 515:108 111. 
Supporting information - Chapter 5

$157 / 168$ 


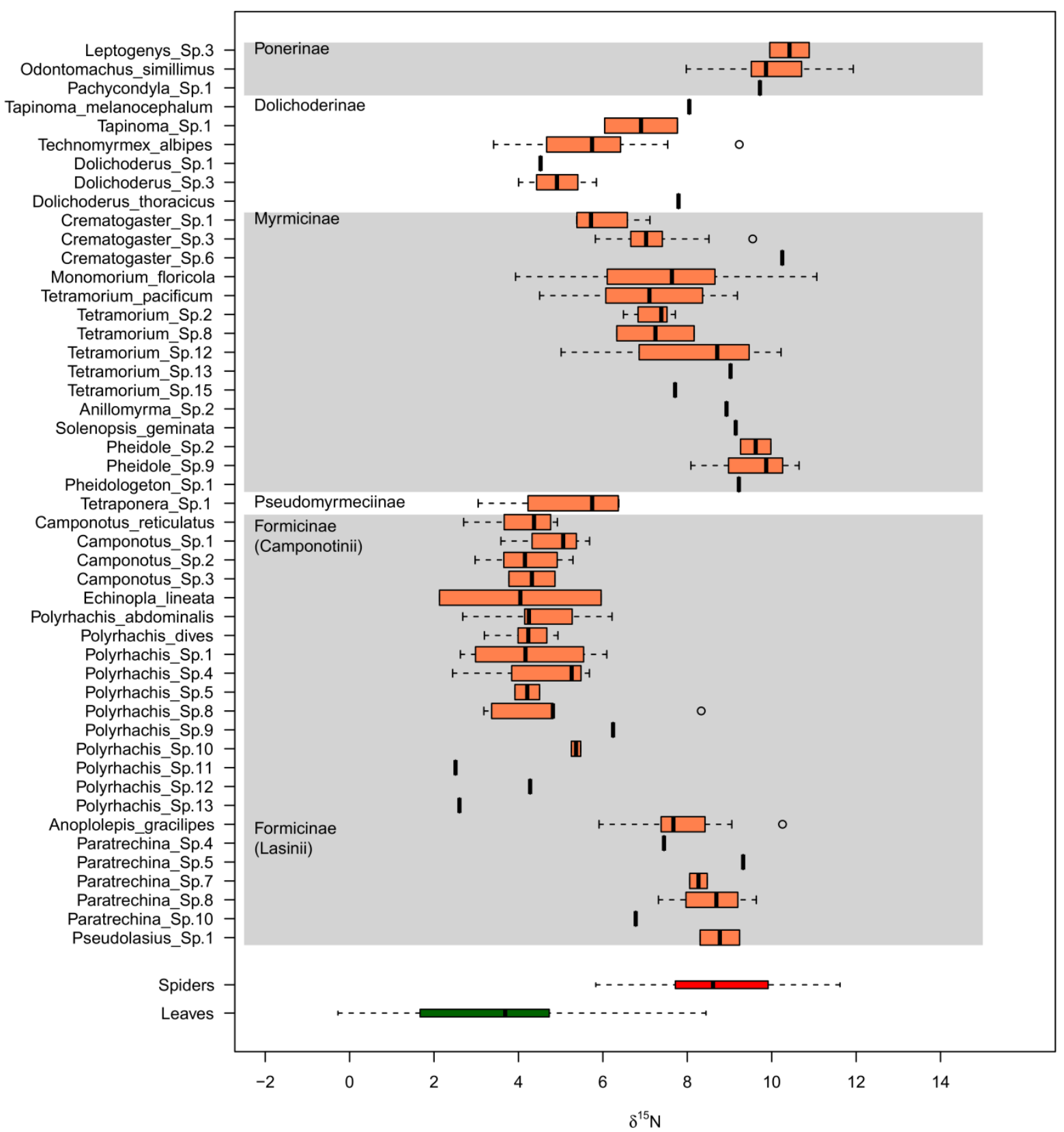

Fig. S1. Variation in delta N between different ant species collected from cacao plantations in Sulawesi, Indonesia. Values for cacao leaves and spiders added for comparison. Ants are presented ordered by family (and tribe in the case of Formicidae). The right axis shows number of samples analysed and number of plantations covered by the samples. 


\section{Thesis summary}

Agroecosystems cover 40 to $60 \%$ of the world's terrestrial ecosystems and agricultural expansion is one of the major reasons of deforestation. Tropical forests harbour most of the organisms worldwide, but agrarian landscapes can also house diverse and abundant communities of organisms. Moreover, agroforests can provide high quality matrices and facilitate animal movement between natural patches. Ongoing agricultural intensification threatens the abundance and diversity of most communities in agroecosystems. Animals of natural and agroecosystems such as ants, birds, bats, and spiders provide ecosystem services such as pest control. Pest control through predation is the direct or indirect result of the predator's trophic role, and the effectiveness of predation can depend on the environment, crop management practices, mutualistic or antagonistic interactions of predators, and competition between species or groups of predators. In this study from the island of Sulawesi (Indonesia), we experimentally analyse the relative role of ant, bird, and bat services for cacao yield and assess the trophic position of cacao ants, switching in their food resource between different trophic levels.

In the first part, we analysed the interactive effects of ants, birds and bats as well as management and landscape context in agroforestry. We implemented a large-scale, full factorial combination of ant, bird, and bat exclusion in Indonesian smallholder agroforestry, along gradients of canopy cover and distance to forest margins. We quantified the contributions of ants, birds and bats to crop yield, the phytophagous and entomophagous cacao arthropods, as well as pest damage, aiming at identifying key drivers of crop protection and cacao yield. We found that the importance of each predator group changed dependent on canopy cover, but not with distance to forest margins. In control treatments, yield was highest under 30-40\% canopy cover. Ant exclusion strongly reduced yield (from 600 to $300 \mathrm{~kg}$ ha-1 year-1) at 15\% canopy cover. Bird exclusion impaired yield (from 400 to $250 \mathrm{~kg}$ ha-1 year-1) at 60\% and enhanced yield (from 600 to $900 \mathrm{~kg}$ ha-1 year-1) at 15\% canopy cover, while bats had no effect. Yield increased with forest proximity, a pattern not related to predator access. No interactive effects among predator exclusions on yield, pest damage and arthropod communities were found. Ant exclusion increased numbers of herbivores below 30\% canopy cover, without reducing spider abundances. Bird exclusion reduced herbivore and increased spider abundances. The complex and context dependent patterns emerging in this study illustrate how little is known of how food web interactions affect crop yield, making general recommendations difficult. Nonetheless, mesopredators can play a crucial role in cacao yield formation, while their effectiveness depends on top predators and plantation 
management. Hence, cacao farmers should refrain from disturbing ant communities and maintain $30-40 \%$ shade-tree canopy cover not only for ecophysiological reasons but also to buffer variability in predator communities.

In the second part, we analysed how the trophic position of two dominant, arboreal ant species, Dolichoderus thoracicus and Philidris cf. cordata, depend on cacao phenology. Omnivorous ants play a plastic and ambivalent trophic role, feeding on plant-derived resources such as honeydew, and arthropod prey, many of them herbivores. Our findings show that density adaptation and trophic plasticity are necessary to compensate spatiotemporal patterns of resource distribution in agricultural landscapes. If food was scarce, both species integrated more honeydew in their diets and colony sizes dropped. Increasing caterpillar abundances resulted in increasing trophic positions and colony sizes, but numbers of available trophobionts influenced colony sizes only. The amount of accessible resources did not correlate with ants' hunting effort, which indicates that predatory services of ants are not directly driven by resource availability. Other influences such as interspecific competition or predator presence may be similarly important. Finally, trophic plasticity and nutritional stoichiometry help to explain why $D$. thoracicus provides ecosystem services preying on herbivores, but P.cf. cordata mainly provides disservices trough cryptic-herbivory and intra-guild predation.

In the third part, we analysed the nitrogen and carbon resource-use of omnivorous ant communities along gradients of canopy cover and distance to forest margins. Omnivores can stabilise trophic webs through preferential consumption of the more abundant resources. However, it is unclear how species-turnover and trophic plasticity contribute to the variability, or conversely the stability, in the trophic role of communities of omnivores across environmental gradients. We used stable isotope analyses to quantify resource-use and found that trophic levels of ant communities decreased under high intensification (low shade tree cover and far from the forest margins). Trophic positions of particular omnivorous ants show similar patterns (P. dives, $C$. reticulatus, $M$. floricola and T. albipes), while other species maintained stable tropic positions along both gradients (A. gracilipes, T. pacificum, O. simillimus). Interestingly, the intraspecific trophic positions - at least partly - influenced the spatial distribution ant species in cacao agroforestry. We infer both, trophic plasticity and species turnover are mechanisms defining the predatory functions of ant communities. Some ant species adapt their trophic position depending on local management and landscape context, while others maintain stable trophic levels. Despite that, strong disturbance at multiple scales can lower predatory function of omnivore communities. Thus, we infer detailed ecological knowledge at species level is mandatory to identify the role of omnivores such as ants in terms of ecosystem services provision. 
In the fourth part, we analysed carbon and nitrogen resource-use of ants, to reveal whether ant communities show systematic trophic shifts to reduce interspecific interference in similar habitats. We used stable isotope $\left(\delta^{15} \mathrm{~N}, \delta^{13} \mathrm{C}\right)$ data from a multi-species, multi-site study as a surrogate for ant trophic level and basal food source, respectively. We revealed that observed nitrogen and carbon isotopic signatures of ants sampled within a community differ more from each other than would be expected by chance. Evidence suggests these shifts are not restricted to feeding on different food sources within a trophic level but can result in a change in the mixture between food sources of different trophic levels. These results demonstrate that the species of an ant community will exploit resources in a complementary way, most likely to minimize costs of interspecific interference. Enhanced complementarity due to trophic shifts could mean that ecosystem functioning may be more stable across heterogeneous, highly diverse communities.

In conclusion, ecosystem service provision of ants and birds is complementary and depends in magnitude as well as in direction on local predator communities, shaped through habitat management. Insectivorous birds can effectively control herbivores, but arthropod predation can turn into a disservice, if easy to capture mesopredators (i.e. web-spiders or trophobiont tending ants) are the predominant prey, which can shape insect communities. Additionally, ant speciesidentity, ant community structure and the local environment (i.e. canopy cover), influence the trophic role of ants, which is also influenced by food resource distribution, local management and landscape context, at least in case of omnivorous ants. Consequently, direct or indirect manipulation of ant communities may be used to increase predation and pest control maximizing crop yields. 
$162 / 168$ 


\section{Danksagung}

Zuerst möchte ich mich bei Teja Tscharntke und Yann Clough bedanken. Vielen Dank für die Möglichkeit, meine Doktorarbeit am Institut für Agrarökologie zu schreiben. Vielen Dank für all die Unterstützung, Zeit und Freiräume, die ihr mir für mein Forschungsvorhaben gelassen habt. Vielen Dank für all das ehrliche Feedback und für jedes Zurückpfeifen, wenn ich mich verzettelt habe. Dir, Yann, möchte ich besonders für Deine Geduld bei stundenlangen Skype-Besprechungen und die großartige Hilfe bei all meinen Fragen danken.

Aijen Tjoa. Vielen Dank für Deinen Einsatz. Ohne Dich stünden meine Proben wohl noch heute in den Regalen in Palu.

Vielen Dank auch an die Organisatoren von ELUC, insbesondere Wolfram Lorenz. Deine Tipps zur Indonesischen Bürokratie und pragmatische Herangehensweise waren Gold wert. Dir, Jutta Gilles, möchte ich dafür danken, dass Du die bürokratischen Hürden in Deutschland für mich gemeistert hast und dabei immer so hilfsbereit und aufmerksam warst.

Vielen Dank an alle helfenden Hände und Freunde in Indonesien. Terima kasih banyak, ke indonesia! Mungkin tidak lama, dan saya punya waktu! ^^^^

Arno, Iris, Yasmin, Andrea und Fabian, euch möchte ich für eine wunderbare Zeit, viel Hilfsbereitschaft und Spaß in Indonesien, und natürlich in Göttingen danken.

Ihr Agrarökologen habt mir das Einleben am Institut erleichtert und jede Rückkehr aus dem Feld herrlich unkompliziert gemacht. Vielen Dank für unzählige Gespräche, Unterstützung und natürlich auch für die gemeinsamen Feiern und die Ablenkungen vom stressigen Berufsalltag. Ich habe die gemeinsame Zeit sehr genossen und wünsche euch allen das Beste für die Zukunft. Ein besonderer Dank geht auch an Verena, Chrissi, Kristy und Hannah für all das leckere Essen. Es war so manches Mal meine letzte Rettung, wenn ich noch bis tief in die Nacht im Büro saß.

Vielen Dank auch an meine Mitbewohner, die mir gerade in den letzten Wochen sehr geholfen haben, mich voll und ganz auf die Doktorarbeit zu konzentrieren.

Ganz besonders möchte ich meinen guten Freunden Jens, Birk und Tilman für viele spaßige Stunden, eure Spontanität und Hilfe in allen Lebenslagen danken.

Marie, ich kann kaum sagen, wie schön es ist, dass wir uns getroffen haben. Aus unserer gemeinsamen Zeit konnte ich in den letzten Monaten unendlich viel Kraft schöpfen. Du bist toll.

Zum Schluss möchte ich meinen Eltern, Jutta und Thomas Gras, danken. Eure bedingungslose Unterstützung und Liebe war eine Voraussetzung dafür, dass ich meine Doktorarbeit schreiben konnte. Ihr habt mich auf meinem Lebensweg sehr beeinflusst, und soweit ich das beurteilen kann - nur zu meinem Vorteil. Ihr seid die tollsten Eltern der Welt! Von ganzem Herzen danke ich auch meinen Geschwistern, Janine und André, für ihre Unterstützung! 
164 / 168 


\title{
Curriculum vitae
}

\author{
Pierre Gras
}

Date of Birth: $25^{\text {th }}$ December 1984

from Peine

Nationality: German

\section{EDUCATION}

since Sept 2015

Dec 2010 - Dec 2014

Oct $2008-$ Nov 2010

Oct $2004-$ Nov 2008
Advanced training: GIS and geodata management specialist. GISAkademie, Hamburg.

Doctoral Studies (Dr. rer. nat.) Thesis: Ecosystem services in agroforestry: predation by birds, bats and ants on spiders and insect herbivores. G.-A. University Göttingen, Department of Crop Science: Agroecology.

MSc: Biodiversity and Ecology Thesis: Aspects of feeding ecology in Siberut Macaques (Macaca siberu), G.-A. University Göttingen. Courant Research center - Evolution of Social Behavior; Final examination: Animal Ecology, Primate Ecology, Agricultural Entomology.

BSc: Horticulture Thesis: Influence of chitosan on attractiveness and host quality of Phaseolus vulgaris for the thrips Frankliniella occidentalis. Leibniz University Hannover, Institute of Horticultural Production Systems: Phytomedicine.

Abitur: Gymnasium am Silberkamp, Peine

FIELD RESEARCH

Feb 2011 - Sep 2012 Indonesia. Universitas Tadulako (Palu, Sulawesi), DFG-Project: Environmental Land-Use Change in Sulawesi, Fieldwork doctoral thesis.

Jan 2010 - Jul 2010 Indonesia. Bogor Agricultural University (Java), SCP Research station (Siberut, Sumatra), Fieldwork MSc thesis.

Sep-Oct 2009 Madagascar. German Primate Center. Field station Kirindy Forest. Practical course: Mutualism or kleptoparasitism - feeding strategy of Dicrurus forficatus.

Jun 2009 Slovenia. G.A Universität Göttingen, Practical course bioacoustics: Migration and fleeing behaviour of Stenobothrus rubicundulus.

Apr 2009 Germany. Alfred-Wegener Institut (Sylt), Practical course Animal ecology: Ecological influence of Littorina littorea on Meiofauna. 
166 / 168 


\section{Thesis declarations}

\section{Declaration of the author's own contribution to manuscripts with multiple authors}

Chapters 2, 3, 4 and 5 are manuscripts prepared for submission to peer-reviewed journals. I am the lead author of manuscripts of chapters 2, 3 and 4, while Yann Clough is the lead author of the manuscript of chapter 5. I personally have contributed to the study design, data collection and statistical analyses for all manuscripts. I have developed the main ideas presented in the manuscripts, written manuscripts 2,3 , and 4 , and created all corresponding tables, figures, and appendices. The co-authors gave advice and contributed to various parts of the study, such as data collection and analyses, discussions and writing. All co-authors contributed to finalizing the manuscripts. In chapter 5, I have written subsections, but overall I took responsibilities as coauthor.

\section{Declaration plagiarism}

I hereby confirm that I have written this doctoral thesis independently, that I have not used sources or facilities other than the ones mentioned, that I have not used unauthorized assistance, and that I have not submitted this thesis previously in any form for another degree at any university or institution.

Göttingen, December 2015

(Pierre Gras) 
$168 / 168$ 